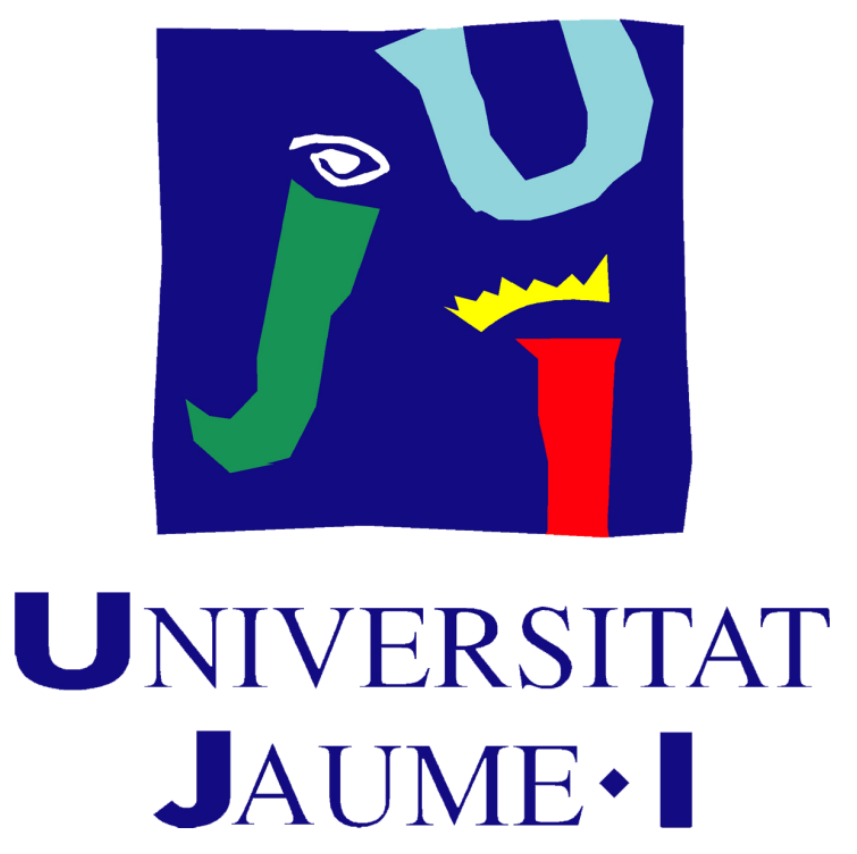

Analysis and Development of a Platform for Generating Context-Aware Apps for Mental Health

Ignacio Miralles Tena

Supervisors:

Carlos Granell Canut Joaquín Huerta Guijarro

November, 2019 


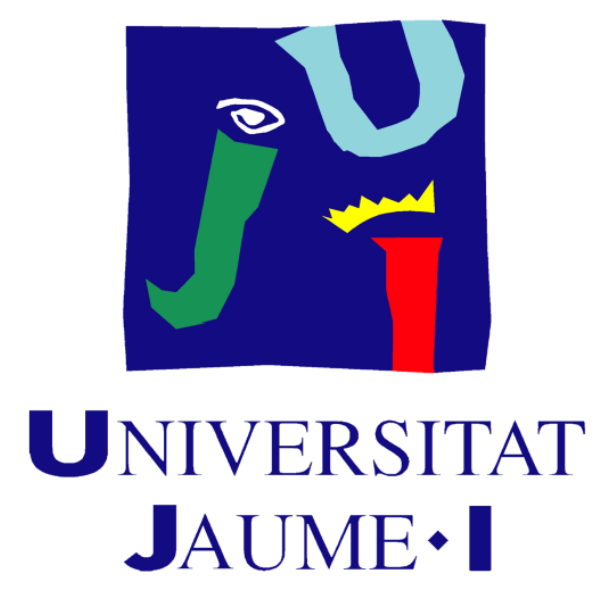

Doctoral Programme in Computer Science Doctoral School from Universitat Jaume I

\section{Analysis and Development of a Platform for Generating Context-Aware Apps for Mental Health}

Dissertation submitted by Ignacio Miralles Tena to apply for the doctorate degree from the Universitat Jaume I

Ignacio Miralles Tena

Carlos Granell Canut

Joaquín Huerta Guijarro

Castelló de la Plana, November 2019. 


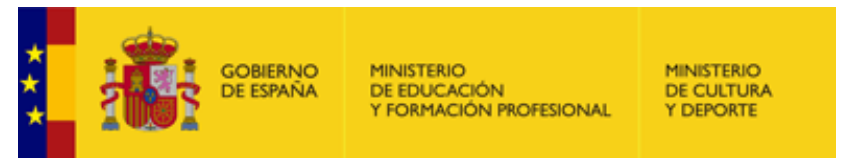

This dissertation is funded by the Spanish Ministry of Education, Culture and Sports, grant reference FPU14/00020. 

Para Juanma, mi primer maestro. 



\section{Acknowledgments}

Mentiría si negara que esta página es la más dura, la que más riesgos conlleva; todas las demás han sufrido doscientas revisiones, esta no tendrá ninguna; las demás sólo serán leídas por expertos y científicos que busquen algunas respuestas, esta sin embargo la leerán las personas más importantes de mi vida. Recordar en unos párrafos tantos años de trabajo no son fáciles, porque este proyecto no empezó hace cuatro años con una beca, posiblemente empezó en 2007, cruzándome con una catedrática en una escalera de un laberíntico edificio de la Universitat Jaume I. Y son demasiadas personas y situaciones las que han contribuido a que hoy se esté escribiendo esto como para que pueda hacerles justicia a todas en unas líneas. Así que, antes que nada: disculpadme aquellos a los que mi memoria haya querido traicionar.

Y si esto va de dar las gracias, tengo que empezar por mis directores. Carlos Granell es el mejor científico que he conocido jamás por tantos motivos que no puedo escribirlos aquí, tenerle de director ha sido una suerte incalculable. Muchas gracias por la paciencia, por la facilidad, por el tiempo y por el esfuerzo que le has dedicado a cada línea y proceso de este trabajo, ha sido un placer y un orgullo aprender de ti todo este tiempo (y sobre todo gracias por la semana de la moda de Milán).

Mi otro director, Joaquín Huerta, se pasa el día peleando para que los más de veinte investigadores y desarrolladores de su grupo puedan seguir creciendo, se parte la cara con quien haga falta para que sepamos que mañana habrá una ayuda, una bolsa, un contrato o cualquier arruche que nos mantendrá a flote otra temporada. Muchas gracias por esa pelea constante.

Gracias a LABPSITEC, el grupo de psicología con el que compartí ocho años de crecimiento y quienes sembraron muchas de las ideas que investigaría años después. En especial a Cristina Botella, quien nunca ha dejado de apoyarme y un 
auténtico faro en el mar de la ciencia, a quién jamás se le podrá agredecer todo lo que le ha dado a la investigación, gracias por ponerme todas las facilidades para que siguiera este camino (un camino que le encanta); a Azucena García, de quien siempre se aprende algo, ya sea trabajando, charlando o tomando una cerveza; a "Ariadna" Mira, porque aunque ella no me pusiera en sus agradecimientos, me ha apoyado tanto que me doy por satisfecho; y por supuesto a Diana Castilla, la criatura envilecida con la que compartí ocho años de sonrisas y lágrimas y de la que tanto he podido aprender, gracias por ser una de las principales responsables de todo esto.

Gracias a GEOTEC, el grupo donde he ejecutado este doctorado, porque me han enseñado una gran cantidad de formas de mirar los mapas y porque me han soportado las chorradas casi cinco años. En especial a Estefanía Aguilar (la Tefi) por aportar siempre un punto de vista nuevo y práctico; a Alberto González (Albertito) porque, aunque no es el consultor que nos merecemos, es el que necesitamos; a Águeda Gómez (UX Expert) porque siempre encuentra tiempo para ayudar, revisar y mejorar cualquier propuesta; a Sergi Trilles (el Concejal), por ser un ejemplo en todo lo que hace; a Sven Casteleyn (Fedrer) porque gracias a su insistencia este trabajo es un poco mejor; y a Aida (Lupillas) porque siempre ha solucionado el problema de desarrollo un par de días antes que tú.

Gracias también a mis padres y a mi hermana, a quienes la vida les debía un doctor.

Gracias a Jorge Osma, la persona con más energía que conozco, por haber confiado en mi cuando ni siquiera cumplía los requisitos.

Gracias a los Buitrélagos, los colegas de toda la vida que me han visto en las duras y en las maduras y que me han aguantado cuando no me lo merecía. En especial al Burren, al Diego, al Miky, al Peter, al Sam y al Yeray.

Gracias al "Dottore" Andrea Calia, amigo, consultor, consejero y amuleto de la suerte, porque me rescató del abismo de la programación y me convenció de que no era tan dificil, por los cafés y las conspiraciones en la sombra y por las cuentas compartidas.

Gracias a Rubén Fernández, qué podría estar en varios de los párrafos anteriores porque ha sido amigo, rival, compañero y familia, porque es de esas personas que siempre están cerca, a las que no tienes que explicarles nada para que te echen una mano, las que te conocen mejor que tú mismo. Porque es un 
orgullo ser amigo tuyo y poder aprender de ti. Y por Vázquez Mella.

A mi hija Valeria, porque me enseña cada día que se puede querer un poco más a alguien y no me deja quedarme quieto ni un instante.

Y a la persona más importante de mi vida, la que hace posible lo imposible, la que consigue que cumpla todos mis sueños, mi constante, el amor de mi vida, a Juani Bretón, gracias por cada minuto que pasas a mi lado. 



\section{Resumen}

Este proyecto de tesis describe el análisis, desarrollo y validación preliminar de una plataforma tecnológica que permite a los terapeutas crear sus propias aplicaciones móviles sensibles al contexto para apoyar las intervenciones psicológicas. El proyecto realiza un estado del arte mediante una revisión sistemática, seguidamente se crea una guía o recomendaciones relevantes para el desarrollo de este tipo de aplicaciones, a continuación se describe el desarrollo de la plataforma tecnológica y, finalmente, se prueba la plataforma con casos de estudio reales. El trabajo está dividido en seis capítulos y su contenido se describe a continuación:

1. El primer capítulo consiste en la introducción general del proyecto, comienza con una motivación del problema y continúa con la descripción de los objetivos generales de la tesis y sus contribuciones.

2. El segundo capítulo es una revisión sistemática sobre aplicaciones móviles utilizadas en los tratamientos psicológicos. Se realiza un estado del arte donde se identifican los tratamientos que se han beneficiado de las tecnologías móviles y las características técnicas de las aplicaciones utilizadas. Este capítulo sirve de motivación para el desarrollo de la plataforma.

3. El tercer capítulo utiliza una metodología mixta para ofrecer una serie de consideraciones que se han identificado como cruciales en el desarrollo de aplicaciones móviles sensibles al contexto en el campo de la salud mental. Se nutre de la información extraída en la revisión sistemática inicial y de las investigaciones realizadas con expertos en el campo.

4. El cuarto capítulo describe el desarrollo de la propia plataforma utilizando como guía un caso ficticio de un paciente con depresión. En este desarrollo se han utilizado como requisitos las conclusiones extraídas tanto de la 
revisión sistemática inicial del segundo capítulo como de las consideraciones generadas en el tercer capítulo.

5. El quinto capítulo se centra en la validación, y describe el uso de la plataforma en la intervención de tres pacientes. Se trata de tres casos de estudio, uno de ellos con pánico y agorafobia y los otros dos con problemas de juego.

6. Finalmente, el sexto capítulo cierra el proyecto mostrando las conclusiones, limitaciones y opciones futuras que presenta la tesis.

Los capítulos del 2 al 5 mantienen el formato de compendio de artículos, siendo cada uno de ellos uno o más trabajos científicos que actualmente están publicados, en revisión o enviados. Por otro lado, la introducción y las conclusiones sirven de guía de lectura y de contextualización general de los capítulos centrales, teniendo una misión de "pegamento" entre los diferentes trabajos o estudios. Cada uno de los capítulos centrales tiene, por tanto, su propia revisión literaria, metodología, resultados y conclusiones. 


\section{Abstract}

This work gathers a research carried out on the use of context-aware technologies in their application to the field of mental health. It begins by performing a deep state of the art of mobile technologies used in psychological interventions and extracting the characteristics and disorders most discussed in the literature; It continues to propose a series of considerations to take into account in the development of this type of technologies to increase its chances of success, focusing on three areas of study: Context, Mental Health and the Technology itself; The next contribution is the process of developing a platform that allows therapists to create their own mobile applications with geolocation to customize their own intervention tools without the need for high technical knowledge, the development is based on the learning obtained in the first two contributions, it is described as an intervention example a hypothetical case of depression; Finally, the platform is validated with three patients suffering from two different disorders: Panic disorder and agoraphobia; and gambling disorder.

Keywords: mental health, mental disorder, intervention, mHealth, smartphone, mobile app, systematic review, place, sense of place, context-aware mobile apps, location-based interfaces, reactive technologogy, angular, panic disorder, agoraphobia, unified protocol, location-based technologies, in vivo exposure, gambling disorder 



\section{Index}

Agradecimientos $\quad$ v

Resumen vii

$\begin{array}{ll}\text { Abstract } & \text { ix }\end{array}$

Acronyms $\quad$ xxi

1 Introduction 1

1.1 A disclaimer before reading this research . . . . . . . . . . . . 2

1.2 Motivation and Problem Description . . . . . . . . . . . . 3

1.3 Objectives and Research Questions . . . . . . . . . . . . . . . 5

1.3.1 Main Objective . . . . . . . . . . . . . . . . 5

1.3.2 Research Questions and Sub-objectives . . . . . . . . . . . 7

1.4 Contributions . . . . . . . . . . . . . . . . . 8

1.5 Thesis Structure . . . . . . . . . . . . . . . . . . . . . 10

1.6 Derived scientific publications . . . . . . . . . . . . . . . . . . . . . 11

1.6.1 Peer-reviewed international conference papers . . . . . . . 12

1.6.2 Peer-reviewed journal articles . . . . . . . . . . . . . . . 13

1.6.3 Related projects . . . . . . . . . . . . . . . . 15

1.6.4 Follow-up predoctoral grants . . . . . . . . . . . . . . . 15

2 Smartphone apps for the treatment of mental disorders: a systematic review. 17

2.1 Introduction . . . . . . . . . . . . . . . . . 18 
2.2 Methods . . . . . . . . . . . . . . . . . . . . . . . 20

2.2.1 Search and Study Selection . . . . . . . . . . . . . 20

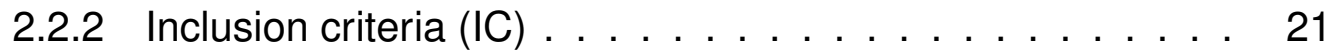

2.2 .3 Exclusions criteria $(E C) \ldots \ldots \ldots$

2.2 .4 Classification of studies . . . . . . . . . . . . . . . 22

2.3 Results . . . . . . . . . . . . . . . . . . . . . . . . 24

2.3.1 Study inclusion . . . . . . . . . . . . . . . . . . . 25

2.3.2 Evolution of research and assessment . . . . . . . . . . 25

2.3.3 Mental disorders addressed . . . . . . . . . . . . . . . . . 28

2.3.4 Relation between assessment type and mental disorder . . 30

2.3.5 Concrete studies per mental disorder and app name . . . . 31

2.3.6 Relation between mental disorder and technical features . 40

2.4 Discussion . . . . . . . . . . . . . . . . . . . . 43

2.4.1 Principal Results . . . . . . . . . . . . . . . . . . . . 43

2.4.2 Coverage of mental disorders . . . . . . . . . . . . . 43

2.4.3 Coverage of technical dimensions . . . . . . . . . . . 44

2.4.4 Assessment types in research studies . . . . . . . . . . 46

2.4.5 Recommendations for the research community . . . . . . 47

2.4 .6 Strengths and Limitations . . . . . . . . . . . . . . . 48

2.4 .7 Conclusions . . . . . . . . . . . . . . . . . . . 49

3 Considerations for designing context-aware mobile apps for mental health interventions. 51

3.1 Introduction . . . . . . . . . . . . . . . . . 52

3.2 Methods . . . . . . . . . . . . . . . . . . . . . 55

3.2.1 Document analysis . . . . . . . . . . . . . . . 56

3.2 .2 Focus groups . . . . . . . . . . . . . . . . . . . . 58

3.2 .3 Assessment . . . . . . . . . . . . . . . . . . . . 60

3.3 Results . . . . . . . . . . . . . . . . . . . 60

3.3.1 Relevant dimensions of each area of knowledge . . . . . 60

Technology . . . . . . . . . . . . . . . . . 61 61

Context ................... 63 
Mental Health . . . . . . . . . . . . . . . . . . 65

3.3.2 Relations between dimensions . . . . . . . . . . . . 66

Technology and Context . . . . . . . . . . . . . . 67

Context and Mental Health . . . . . . . . . . . . . 69

Mental Health and Technology . . . . . . . . . . . . 71

3.4 Discussion . . . . . . . . . . . . . . . . . . . . 72

3.4 .1 Considerations . . . . . . . . . . . . . . 73

3.4 .2 Limitations . . . . . . . . . . . . . . . . . . . . . . 75

3.5 Conclusions . . . . . . . . . . . . . . . . . . . 75

4 Development of a builder tool for customizing location-based, gamified apps for depression. $\quad 77$

4.1 Introduction . . . . . . . . . . . . . . 78

4.2 Methods . . . . . . . . . . . . . . . . . . . 81

4.2.1 Software engineering methods . . . . . . . . . . 81

4.2.2 Qualitative methods for requirement elicitation . . . . . 82

4.3 Results . . . . . . . . . . . . . . . . . . . . . . . . 84

4.3.1 The builder tool . . . . . . . . . . . . . . . . . . . . 84

4.3.2 The mobile app . . . . . . . . . . . . . . . . . 88

4.3.3 The viewer tool . . . . . . . . . . . . . . . . . . . . . . . . 89

4.3.4 Technological infrastructure . . . . . . . . . . . 90

4.4 Discussion . . . . . . . . . . . . . . . . . . . . . . . . . . 94

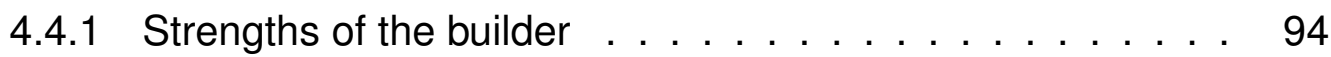

4.4.2 Limitations and future perspectives . . . . . . . . . . . 95

4.4 .3 Conclusion . . . . . . . . . . . . . . . . 95

5 Validation and experimentation of the platform in case studies with real patients.

5.1 Enhancing in vivo exposure in the treatment of panic disorder and agoraphobia using location-based technologies: A case study. . . 98

5.1 .1 Introduction . . . . . . . . . . . . . . . . . . . 99

5.1 .2 Method . . . . . . . . . . . . . . . . 102 
Patient . . . . . . . . . . . . . . . . . . . . 102

Therapist . . . . . . . . . . . . . . . . . 103

Measures . . . . . . . . . . . . . . . . . . 103

Treatment and IVE . . . . . . . . . . . . . . . . . . . . 104

System description . . . . . . . . . . . . . . . . . 105

Builder . . . . . . . . . . . . . . . . . . . . . . . . . . 105

App . . . . . . . . . . . . . . 106

Viewer . . . . . . . . . . . . . . . . . 106

Design and procedure . . . . . . . . . . . . . 110

5.1 .3 Results . . . . . . . . . . . . . . . . . . . . . 111

Target Behaviors . . . . . . . . . . . . . . . . . . 111

Expectations and Satisfaction regarding the IVE+LBT . . . 111

Satisfaction and usability of the IVE+LBT . . . . . . . . . 112

5.1 .4 Discussion . . . . . . . . . . . . . . . . . . . . . . . 114

5.2 Enhancing therapeutic components in the treatment of gambling disorder using location-based technologies . . . . . . . . 118

5.2 .1 Introduction . . . . . . . . . . . . . . . . . . . 118

5.2 .2 Method . . . . . . . . . . . . . . . . . . . . . . 122

Patients . . . . . . . . . . . . . . . . . 122

Therapist . . . . . . . . . . . . . . . . . . . . . 123

Measures . . . . . . . . . . . . . . . . . . . . . . . . . . . 124

Treatment . . . . . . . . . . . . . . . . . . 125

System Description . . . . . . . . . . . . . . . . . 129

Design and Procedure . . . . . . . . . . . . . . . . 129

5.2 .3 Results . . . . . . . . . . . . . . . . . . . . . . . . 130

5.2 .4 Discussion . . . . . . . . . . . . . . . . . . . . . . . 134

6 Conclusions 139

6.1 Main research contributions . . . . . . . . . . . . . . . . . 139

6.2 Limitations . . . . . . . . . . . . . . . . . . . . . . 142

6.3 Future Work . . . . . . . . . . . . . . . . . . . . . . . 144 
$\begin{array}{ll}\text { A Appendix } & 187\end{array}$

A.1 Supplementary Material A: Keywords . . . . . . . . . . . . . . 187

A.2 Supplementary Material B: Search Queries . . . . . . . . . 188

A.2.1 SCOPUS search . . . . . . . . . . . . . . . . . . 188

A.2.2 WoS searches . . . . . . . . . . . . . . . . . . . 189

A.2.3 MEDLINE searches . . . . . . . . . . . . . . . 190

A.2.4 APA PsycNET search . . . . . . . . . . . . . 190 

xvii

\section{Index of tables}

2.1 Mental disorders and the studies targeting them. . . . . . . . . . . 32

3.1 Sociodemographic data of the participants of the focus group one. 59

3.2 Sociodemographic data of the participants of the focus group two. 60

3.3 Summary of selected dimensions grouped by area of knowledge. . 66

4.1 Considerations for designing context-aware mobile apps for mental health interventions . . . . . . . . . . . . . . . 84

5.1 Expectations and satisfaction with IVE+LBT. . . . . . . . . . . . . 113

5.2 Usability test about IVE+LBT. . . . . . . . . . . . . . . . . . . 113

5.3 Treatment's content. . . . . . . . . . . . . . . . . . . . 127

5.4 SC/ERP+LBT usability test for patient 1. . . . . . . . . . . . . . . 132

5.5 SC/ERP+LBT usability test for patient 2. . . . . . . . . . . . . . . . 134 



\section{Index of figures}

2.1 PRISMA flow diagram for the systematic review. . . . . . . . 25

2.2 Temporal trend of amount of articles published and assessment type. 27

2.3 Distribution of articles per mental disorder. . . . . . . . . . . . . . 29

2.4 Distribution of articles published for the top 6 mental disorders over time. . . . . . . . . . . . . . . . . 30

2.5 Bubble plot representing technology-related dimensions. . . . . . 42

3.1 Qualitative research methods in relation to the Research Questions. 55

3.2 Areas of knowledge and dimensions. . . . . . . . . . . . 61

3.3 Relationships between dimensions. . . . . . . . . . . . . . . . 67

3.4 The design process of context-aware mobile apps. . . . . . . 72

4.1 Focus group. $\ldots \ldots \ldots \ldots \ldots \ldots$

4.2 Flowchart diagram of the process to create a new application. . . . 86

4.3 Screenshots of the built application. . . . . . . . . . . . 89

4.4 Screenshot of the visual interface of the configuration. . . . . . . 90

4.5 Functional architecture of the platform. . . . . . . . . . . . 91

5.1 Screenshot of the Create Patient form. . . . . . . . . . . 106

5.2 Screenshot of the New Place form. . . . . . . . . . . . . . 107

5.3 AppKey Request Screenshot. . . . . . . . . . . . . . . . . . . 108

5.4 Screenshot of the viewer. . . . . . . . . . . . . . . . . . 108

5.5 Technological Architecture of the Symptoms Platform. . . . . . . 109

5.6 Located target behaviors with fear and avoidance rating. . . . . . 112

5.7 Overwhelming urge to play slot machines for patient $2 \ldots \ldots$. . . 131 
5.8 Appettive urge to playing slot machines (0-10) for patient 2. . . . 131

5.9 Expectation and satisfaction with the SC/ERP+LBT by patient 1 . . 132

5.10 Expectation with the SC/ERP+LBT by patient $2 \ldots \ldots \ldots$ 


\section{Acronyms}

ADHD Attention-Deficit Hyperactivity Disorder

ADIS Anxiety Disorders Interview Schedule

APA American Psychology Association

CBT Cognitive-Behavioural Therapy

cCBT Computerized Cognitive-Behavioural Therapy

DGOJ Directorate General for the Regulation of Gambling

DSM Diagnostic and Statistical Manual of mental disorders

EC Exclusion Criteria

EMA Ecological Momentary Assessment

EMI Ecological Momentary Intervention

ERP Exposure with Response Prevention

GD Gambling Disorder

GEOTEC Geospatial Technologies Research Group

GPS Global Positioning System

IC Inclusion Criteria

ICT Information And Communication Technologies

IVE In-Vivo Exposure 
LABPSITEC Laboratorio de Psicología y Tecnología

LBT Location-Based Technologies

NODS National Opinion Research Center DSM-IV Screen for Gambling Problems

NPC Non-Playing Character

PD Panic Disorder

PD/A Panic Disorder/Agoraphobia

PG Pathological Gamblers

PHQ Patient Health Questionnaire

RCT Randomized Controlled Trial

REST Representational State Transfer

RQ Research Question

SC Stimulus Control

SUS System Usability Scale

UI User Interface

UJI Universitat Jaume I

UP Unified Protocol

UX User Experience

VR Virtual reality

WHO World Health Organization 



\section{Introduction}

Fly, you fools!.

Gandalf, the grey.

This introductory chapter provides a global context of the research work conducted in this doctoral thesis. It begins with some practical recommendations on how to best read this multidisciplinary manuscript, followed by the description of the problem statement that drove the motivation of the present research work. Next, the chapter introduces readers in the main objective of this thesis along with the particular sub-objectives and research questions that have been addressed in the course of the research. This chapter continues with a brief summary of the main contributions to anticipate readers with the scientific results obtained from this thesis. The last part provides additional information on the structure of the entire document, linking the different chapters with the sub-objectives and research questions raised before. Finally, scientific works and research projects emerged over the past years during the development of this doctoral thesis are enumerated and briefly contextualised. 


\subsection{A disclaimer before reading this research}

Before beginning the reading of this research it would be convenient to have an overview of it. This thesis addresses some particular problems in interventions for mental disorders from a technological perspective. It identifies a need, analyses existing possibilities, develops convenient tools and applies them to patients. Therefore, this thesis necessarily involves multidisciplinary research that includes diverse aspects from the fields of Computer Science and Psychology.

This circumstance makes it a deeply enriching investigation in many aspects; however, it poses some limitations or particularities that should be kept in mind when reading this manuscript.

Some sections such as methodology (or methodologies) and the description of results do not fully align to the established organisation and structure of either of the two disciplines; they, however, combine mixed structures to fully meet the particular needs of multidisciplinary research. Consequently, a computer scientist (or psychologist) can find the reading of these chapters a bit weird, but at the same time and on the positive side, he/she will be exposed to other forms of scientific writing and scientific content other than the main speciality.

The content of the thesis (from chapter 2 to chapter 5 ) is a compendium of published scientific works that describe different phases of the research work. Following the standard convention of scientific articles, each of these works, and hence the corresponding chapters, has the same structure of main sections: introduction, methodology, results and discussion. However, they all are part of the same plot that will be described later in this chapter. A reader can focus on an individual work (chapter), as each one is a separate piece of research, or read them in sequence. The present chapter and the last chapter (Chapter 6) serve as a common background, like "glue" that wraps the central chapters and gives meaning to the research project as a whole.

Finally, the author would like to make a metaphor for this research with the fantasy novels that have accompanied him throughout his life. In these novels, there is generally a dark and growing force whose goal is to bring great evil to the world. There is also a small resistance of warriors or wise men and women who face it with very few possibilities and resources, usually the protagonists. In that metaphor, mental disorders are that dark force that is increasingly threatening; the 
resistance is the therapists and psychologists who, with their research and treatments, try with great difficulty to stop it. And where are the computer scientists? In the forges, the hammer and the anvil rumble as they create the weapons, swords, spears and axes with which the warriors will go out to face danger. We (computer scientists for "social good") attempt to develop the most appropriate technology and tools for the needs of people, patients and therapists, so that little by little the darkness is giving way.

I hope the reader enjoys this work as much as the author did in doing it.

\subsection{Motivation and Problem Description}

In our modern society, mental disorders are a worldwide burden as identified in multiple studies. For example, a 2019 report of the World Health Organisation (WHO) [1] states that 83 million citizens of the European Union suffered some mental disorder in 2018; in Trautmann et al. [2] it is indicated that about 165 million people have been diagnosed a mental disorder at least once in their lives. The overwhelming of these figures worsens when we see the trend: Vos et al. [3] concluded that the number of people with depression has increased $20 \%$ worldwide from 2005 to 2015.

From a human perspective, this is clearly a huge problem since people's health and well-being are simply degraded. But it has other dimensions if we analyze the monetary costs of mental disorders. Evans-Lacko et al. [4] wrote that the cost for Europe in 2010 was 72 billion euros. The data extracted from Trautmann et al. [2] is even worse, speaking of 2.5 trillion US Dollars a year.

One of the aims of the broad field of Psychology is to deal with this problem by studying, exploring, experimenting and applying effective treatments and protocols. Unfortunately, there are only 4.6 psychologists per 100,000 inhabitants in Europe, according to the mental health workforce breakdown by WHO region [5], which gives an idea of the enormous effort that a professional must make to ensure that each potential patient is the best treated, which obviously is not always feasible. Indeed, if we look on the national level, recent studies have reported waiting times of more than 45 days before the first psychological assistance by a clinical psychologist or psychiatrist [5], and the frequency of face-to-face sessions limited to once per month [6].

The issues and problems mentioned above can be summed up in that there is a 
lack of resources available for a painful and costly problem that affect a significant part of the society. Therefore, we suggest that the more resources psychologists have at their disposal, the better the care and treatment will be for patients suffering from mental disorders.

Among the resources and tools that might help alleviate this problem, the use of technology is perhaps one of the most promising. Over the recent years there has been an upward trend in the use and integration of technologies in psychological interventions. There are multiple works that have proven useful for this. Starting with the pioneering works with virtual reality (VR), which proved effective for example in anxiety disorders [7-13] and continuing with the broad use of Internet as a means to spatially decouple patients from therapists [14-17]. Mobile devices and smartphones [18] are one of the recent attempts to combine mobile technology into psychological interventions. Their popularity has exponentially increased in the last ten years, with data that speak of $35 \%$ of the world population that carries one of them in their pocket, being this proportion widely exceeded in developed countries (76\% in the United Kingdom, or 95\% in South Korea) [19, 20].

Smartphones have a number of features that encourage their use in mental health. They are versatile, mobile, can be customized, and their computing capacity enables them to perform data analysis in situ and run sophisticated virtual environments in a precise and powerful way. Smartphone and mobile devices are in general no intrusive (Ecological), since they are already part of people's lives, and can be used virtually anywhere and at any time (Momentary). The use and application of smartphones to interventions has allowed patients to be constantly monitored so that their symptoms can be evaluated regularly. As a result, psychoeducation content delivered through smartphones has already been explored in interventions [21]. All in all, the possibilities that bring smartphones and mobile devices to mental disorders interventions seem endless, and even new functions and innovative uses continue to appear today [22-28].

This situation undoubtedly presents a promising connection between mental health and technology, however it is not without problems and gaps that must be carefully explored.

On one hand we have the connection and communication between the disciplines of Computer Science and Psychology. In general, engineering teams are disconnected from the needs of psychologists, and the latter are in turn unaware of the working mechanisms of engineers, as well as the full range of possibilities 
that technology can bring to them. In addition, the design and development of effective interventions often entails high costs in development tools and human resources, and this is not always acceptable or possible by clinical teams. On the other hand, even when these communication barriers are overcome, developments of smartphone applications are often limited to a few basic, specific features or needs that the application must meet, without considering the complexity of the interrelated considerations and features as a whole that can take part of an intervention to make it a success. For example, they lose sight of the usability part of the application for the patient, scarcely account for the addition of adhesion components, and pay little or no attention to the visualisation of clinical content in a way that suits the environment and the context in which that content will be consumed.

The present work precisely puts emphasis on the use of the context and the environment to design and create better tools for mental disorder interventions. In similar health-related scenarios, context matters a lot. For example, environmental health experts have experimentally confirmed the importance of the geographical context in health by finding strong correlations, for example, between high levels of noise or pollution and degradation of well-being [29, 30]. The distribution of the urban environment itself is also another important factor to consider, because it directly influences people's health [31-34].

Therefore, the three core areas on which this research is based come out: the mental health of people, the technology to support treatments, and the context where these treatments are going to be applied to people.

We do not include a state of the art of each of these areas here. We indeed refer readers to the background sections of subsequent chapters where these areas are extensively covered. In doing so, we make the introduction more concise and avoid excessive redundancy.

\subsection{Objectives and Research Questions}

\subsubsection{Main Objective}

The main objective of this research work is to expand the catalog of technological tools at the disposal of mental health professionals to carry out their psychological evaluations and interventions. In this thesis, these tools refer specifically to context- 
aware mobile applications that meet the ecological and momentary requirements and take advantage of the patient's context to facilitate and maximize the work of the clinician.

At this point an introductory and motivating example will definitively clarify what we mean by "expanding the catalog of technological tools" and, consequently, will lead us to the identification and understanding of the proposed research questions.

Imagine John, a patient diagnosed with panic and agoraphobia disorders who visits her therapist's office for the first session. The therapist, Margaret, accesses to the (Symptoms) website and registers John as a patient. Then, Margaret creates a new application for her new patient. Given John's diagnosis, she specifies through the website a list of feared places (a shopping mall, a specific crossing or any other place on the map that is relevant to the diagnostic) close to or within the John's neighborhood. In addition, Margaret adds additional information that's clinically relevant to each of these places. For example, a motivating message, notification or any other clinical content the Margaret consider crucial when John approaches to that place. Once all places on the list are configured, Margaret proceeds with the generation of the mobile application. At this point, the website also creates a private code for John to be able to install the (Symptoms) mobile application on his mobile phone by entering that security code. John's gone and back home. From that moment, every time John approaches one of the indicated places, he will be prompted with the information indicated by Margaret. During the treatment, Margaret will be able to monitor John's steps and notifications received by only accessing to a website from her office. Real-time monitored data will therefore inform Margaret about John's trajectories and movement around the city, how many times he visited each feared places and the messages he received.

This is only an imaginary example, of course, but the real chances are much greater because the stakes are high as well. If, instead of being feared places to which the patient is encouraged to get closer, they were prohibited places, whose visit must be deterred (for example, betting centers for patients with pathological gambling disorder), the mobile application will remain exactly the same with the exception of modifying the contextual information (i.e., configuration) delivered in each place. The application also allows to monitor depressive patients, motivating them every time they leave home or walk a certain distance on foot. And it even allows to launch specific questionnaires according to contextual triggers (when patients are near a place, every so often, when a certain time passes without 
detecting movement, etc.). These are just some of the ideas of what the developed tool can do, although, as a specifically designed tool to support extensibility, new features and functions can be straightforward added into the tool.

\subsubsection{Research Questions and Sub-objectives}

The overarching objective of expanding the tools with which therapists can cope with the diversity and complexity of mental health treatments can be divided into sub-objectives that are in turn directly related to the research questions that drive this thesis. Therefore, in what follows, we describe each sub-objective and the associated research question to address the main objective.

- Objective 1 (01): What has been done so far regarding the application of mobile devices and apps to psychological interventions? Our intent is to know, on the one hand, the range of mental disorders that have been treated by mobile technologies and, on the other hand, to identify the characteristics of these technologies that have been used to a greater or lesser extent. Despite the wide use and penetration of mobile technology and smartphones, our initial belief is that their technical possibilities are still widely underused. As we will see in Chapter 2, the majority of the surveyed mobile apps are designed to transfer the classical methods (of pencil and paper) in psychological treatments to the digital medium (mere digitization), without taking advantage of the possibilities that this new medium offers.

The associated Research Question 1 (RQ1) is: what is the current state of the art in the use of mobile applications for psychological interventions? This question can be read in two ways:

- What are the mental disorders that have been subject to mobile applications?

- What are the technological features that have been most used? And what for?

- Objective 2 (02): How should a mobile app be designed for effective intervention? This objective aims to identify a series of relevant dimensions in relation to the three core areas seen in Section 1.2 (technology, context and mental health) that any design of technological development for interventions 
should take into account. As we will see in Chapter 3, aspects such as adherence, usability and the characteristics of the contextual environment appear will be necessary.

The associated Research Question 2 (RQ2) is: what are the design considerations that must be taken into account so that a technological development applied to psychological interventions maximizes the chances of success?

- Objective 3 (03): To develop a technological platform that account for everything previously learned. In Chapter 4, we deep into the development and design of a platform to allow mental health experts create their own technological interventions without losing sight of the outcomes of the first two objectives.

The associated Research Question 3 (RQ3) is defined as: can we develop a technological platform that allows therapists to create their custom mobile applications for psychological interventions in a simple way and without the need for technological knowledge?

- Objective 4 (04): To validate the technological platform in real settings, i.e. as part of treatments with real patients diagnosed with different mental disorders. Experimentation will be the focus of Chapter 5 .

The associated Research Question (RQ4) is: does the technological platform support psychological treatments for distinct mental disorders?

The four research questions are satisfactorily addressed in the central chapters (2-5) of the thesis, in the same order as listed above. First, an in-depth, rigorous state-of-the-art study on the subject is carried out (O/RQ1); a series of considerations are then drawn up for the design of technical developments (O/RQ2): next, a technological platform is developed by integrating all the previous outcomes (O/RQ3); and, finally, the feasibility and effectiveness of the platform and mobile applications are assessed in psychological interventions with real patients (O/RQ4).

\subsection{Contributions}

From a tangible point of view, the main contribution of this doctoral thesis is the technological platform called Symptoms. The Symptoms platform does 
play the role of the website tool in the introductory example in Section 1.3. It is a functional, operational, web-based platform that allows therapists, whether or nor they are tech-savvy, to create their own mobile applications adapted to the psychological interventions in question. Whereas the platform itself can be considered an engineering contribution, the contained elements on which the platform is built are important themselves and, as such, we consider them as valuable scientific contributions to advance in the field. Below, we summarize the main engineering and scientific contributions of this thesis.

- Engineering contributions: The Symptoms platform is the major contribution of this thesis, along with the generated mobile applications which interact directly with the patients.

- Scientific contributions: A combined use of the following minor contributions makes significant progress to the field.

- Firstly, an updated state of the art on the use of mobile applications in mental health informs on the technical characteristics used, the actual trends and the most studied disorders.

- Secondly, a series of design considerations are explored and formalised, leading to a theoretical model on top of which context-aware mobile applications can be designed for conducting effective interventions.

- Finally, experimentation through two case studies permitted us to both check the validity of theoretical model and the acceptability of the platform.

The above contributions are directly related to each of the sub-objectives and research questions of the thesis (Section 1.3). However, it is worth to note here that this thesis attempts to provide tools for mental health that have not yet been exploited, to the best of our knowledge, in psychological interventions, under the prism of two remarkable angles:

On one hand, the platform and mobile apps capture and bring contextual information and spatial concepts to interventions such as location, trajectories, proximity, distance traveled, and particular characteristics of relevant places. Are they feared places? Are these recommended places? What relationship does the patient have with a specific place or with the specific characteristics of the place? Although 
there have been works before, as will be seen in Chapter 2, we claim that, when designing context-aware mobile apps for psychological interventions, this contextual and spatially-related information and its relationship with the patient has not been explored enough. Therefore, we expect that the findings of this thesis can open the door to future improvements in context-aware developments that can dynamically adapt to the changing peculiarities of patients and their surrounding environment.

On the other hand, it is expected that the platform can become a game changer to accelerate the development process and deployment of mobile applications. Leaving apart the technological barriers and issues related to finding and economically covering technical teams for developing and personalizing mobile tools, it will allow mental health professionals to focus on the best psychological framework for their interventions and treatments. Our long-term expectation is to foster a mindset shift on the current perception of technology from an impediment that slows down psychologists' work to valuable, trusted, and ready-to-use tools that guarantee objective and updated information, adherence and concretion when carrying out interventions.

\subsection{Thesis Structure}

As earlier commented, this thesis is organised as a compendium of published scientific articles. In particular, all but Chapter 1 and Chapter 6 corresponds to scientific publications, either published (Chapters 2 and 3) or under review (Chapters 4 and 5 ) at the time of writing. A reader will find the following six chapters:

- Chapter 1 Introduction. This chapter introduces the motivation and problem statement, the main objective and research questions, summarises the major contributions, and briefly describes the relevant scientific publications stemmed directly from this research .

- Chapter 2 Smartphone apps for the treatment of mental disorders: a systematic review. This chapter covers O1 (RQ1). It contains a systematic review to analyse the most confronted disorders through mobile technologies and the salient characteristics of these technologies that have been used. 
- Chapter 3 Considerations for designing context-aware mobile apps for mental health interventions. This chapter covers $\mathrm{O} 2(\mathrm{RQ} 2)$. It is a guide of aspects (or a series of considerations) to consider for the design of mobile applications for psychological treatments.

- Chapter 4 Development of a builder tool for customizing location-based, gamified apps for depression. This chapter covers O3 (RQ3). It fully describes the development of the technological platform, using as a running example a case of depression disorder.

- Chapter 5 Validation and experimentation of the platform with real patients. This chapter covers O4 (RQ4). It goes step by step in the validation experiment of the platform as a support tool in the treatment of patients. Three patients have been treated using the platform and are conveniently described in this chapter: one patient with panic disorder and agoraphobia, and the other two with gambling disorder.

- Chapter 6 Conclusions. The final chapter summarizes the main achievements of the present thesis, followed by the limitations of the research, and concludes with reflections on future research lines in the short and medium term.

The publication-driven chapters, given their compendium structure, present their own scientific contextualization, methodology, and describe the related findings, results and conclusions. Next, the list of scientific publications are briefly annotated, providing therefore a summary of each of the corresponding chapters.

\subsection{Derived scientific publications}

A host of scientific publications has been produced in the course of the research work. Some of them are the direct result of research work conducted within the GEOTEC (Geospatial Technologies Research Group) group. The author of this thesis presented some in international conferences and forums. One remarkable aspect is that, based on the initial findings of this thesis, funding has been obtained through follow-up projects and predoctoral fellowships at regional and national levels. The author of this doctoral thesis has collaborated to a greater or lesser extent in each and every one of these scientific publications, being, in many of 
them, the first author or principal investigator, while in others he has taken a secondary role. For all items listed next, we briefly point out their connection to this thesis.

\subsubsection{Peer-reviewed international conference papers}

- Miralles, I., Granell, C., and Huerta, J. (2016). Playability Index, Built Environment and Geo-Games Technology to Promoting Physical Activity in Urban Areas. In Ubiquitous Computing and Ambient Intelligence (pp. 437-444). Springer, Cham.

This work identified the list of urban characteristics that were perceived as the most influential in the use of context-aware mobile applications to promote physical activity. In addition to giving a general context about the urban environment and health, it is the embryonic stage of the design considerations finally discussed in Chapter 3 .

- Miralles, I., Granell, C., Rodríguez-Pupo, L. E., Casteleyn, S., and Huerta, J. (2017, October). Games, health and the city: Developing location-aware games for leveraging the most suitable places for physical activity. In Extended Abstracts Publication of the Annual Symposium on Computer-Human Interaction in Play (pp. 239-245). ACM.

In this work, the behavioral activity of people is connected with the environment in which they are. It extends the work carried in the previous paper ("Ubiquitous Computing and Ambient Intelligence") and represents an step forward in the conceptualisation of the design considerations. Therefore, it also links to Chapter 3.

- González-Pérez, A., Miralles, I., Granell, C., and Casteleyn, S. (2019, September). Technical challenges to deliver sensor-based psychological interventions using smartphones. In Proceedings of the 2019 ACM International Joint Conference on Pervasive and Ubiquitous Computing and Proceedings of the 2019 ACM International Symposium on Wearable Computers (pp. 915-920). ACM.

The focus of this workshop paper is the description of the conceptual architecture and defining technological characteristics of the Symptoms platform. It aims to raise and discuss, in an international forum, the challenges and 
potential solutions for this type of applications. It entirely relates to chapter 4, which describes the technological part of the development of the platform.

- Diaz, L., Miralles, I., Granell, C., Bretón-López, J., González-Pérez, A., Casteleyn, S., Castilla, D. and García-Palacios, A. Enhancing stimulus control in the treatment of gambling disorder using location-based technologies. In: The 6th ESRII Conference. 2019.

This work examines the use of the Symptoms platform in patients with game disorders. In this case the intended use of the platform is to strengthen the control stimuli and facilitate exposure with response prevention. It relates directly to chapter 5 , given that it entails a new experiment that serves as a validation of the technological platform.

\subsubsection{Peer-reviewed journal articles}

At the time of writing this document there is one published paper, three sent (one of them under review and two awaiting a first decision) and a fifth in preparation. Marked in bold, the scientific publications in which the doctoral student is the first author and, therefore, are part of this doctoral thesis. Although relevant and referenced here, the remaining publications are not included in this thesis because their content is not entirely derived from this research.

- Miralles, I., Granell, C., Díaz, L., Van Woensel, W., Bretón-López, J., Mira, A., Castilla, D. and Casteleyn, S. Smartphone apps for the treatment of mental disorders: a systematic review. JMIR Preprints. 03/06/2019:14897

This work literally corresponds to Chapter 2 of this thesis. It is in the first revision to the high-profile journal in digital medicine and e-health, with an impact factor of 4.945 (Q1, top1).

- Miralles, I. and Granell, C. (2019). Considerations for Designing ContextAware Mobile Apps for Mental Health Interventions. International journal of environmental research and public health, 16(7), 1197.

This work literally corresponds to chapter 3 of this thesis, published in the "International Journal of Environmental Research and Public Health" (IJERPH), with an impact factor of 2.468 (Q2). 
- González-Pérez, A., Casteleyn, S., Miralles, I., Bretón-López, J., Castilla, D. and Granell, C.. SyMptOMS - A Sensor-based Mobile Mental Health platform.

This work is related to chapter 4 of this thesis. It describes aspects of the technological platform, but goes beyond the platform initially investigated and tested in the present doctoral thesis. However, its fundamental part has its origin in the research done here. It is under review in the IEEE Internet Computing, with an impact factor of 2.891 (Q1).

- Miralles, I., Matey, M., Granell, C., Gónzalez-Pérez, A., Bretón-López, J., Castilla, D., Rebollo, C. and Casteleyn, S. Development of a builder tool for customizing location-based, gamified apps for depression.

This work literally corresponds to Chapter 4 of this thesis. It describes the technical characteristics of the platform using as a guide the hypothetical use case of a patient with depression. It has being adapted to the specification of the target journal, the International Journal of Medical Informatics (IJMI), with an impact factor of 2,731 (Q2), so it does resembles exactly the organisation of the Chapter 4.

- Miralles, I., Granell, C., García-Palacios, A., Castilla, D., Gónzalez-Pérez, A., Casteleyn, S. and Bretón-López, J. Enhancing in vivo exposure in the treatment of panic disorder and agoraphobia using location-based technologies: A case study.

This work literally corresponds to section 5.1 of chapter 5 of this thesis. It describes the use of the platform with a real patient suffering of panic and agoraphobia. It is under review in the Clinical Case Studies, an international journal with an impact factor of 0.867 (Q4).

- Miralles, I., Bretón-López, J., Granell, C., Díaz, L., Mira, A., GónzalezPérez, A., Casteleyn, S. and García-Palacios, A. Enhancing therapeutic components in the treatment of gambling disorder using location-based technologies: A case study of two participants' experiences.

This work literally corresponds to section 5.2 of chapter 5 of this thesis. It describes the use of the platform with two patients suffering of pathological gambling. At this time this document is in the final phase of its preparation for sending to a specialized journal. 


\subsubsection{Related projects}

Based on this research, two research projects have been granted and funded by local and national agencies, namely:

\section{- AnyTIME: Apps To Improve Mental hEalth}

This is an internal project of the Institute of New Imaging Technologies (INIT), aimed to foster collaboration between the different research sections of the institute. The GEOTEC section, author's research group, and the Interactive Visualization section teamed up in the proposal AnyTIME, which essentially was aimed to add gamification and adherence components to the platform. The results of the project are partially shown in the chapter 4 , where the technological platform is described. The project got $€ 3,500$ and the Principal Investigator was the author of this thesis.

- SyMptOMS: Sensor and MObile based Mental Health Solutions

This is project funded by the 2018 "Programa Estatal de I+D+i Orientada a los Retos de la Sociedad", from the "Ministerio de Ciencia, Innovación y Universidades" (reference number RTI2018-099939-B-I00). The objective is to develop innovate solutions for the diagnosis, treatment, and relapse prevention of mental health disorders. This national project sits on the first results obtained in this research to explore and implement advanced data analysis capabilities in the Symptoms platform.

\subsubsection{Follow-up predoctoral grants}

This work is fully covered by a predoctoral FPU grant obtained by the author of this thesis from the Spanish Ministry of Education, Culture and Sports (grant reference FPU14/00020). In addition to this, further developments of this research led to extended and novel features that were central to getting two predoctoral grants. Next, we indicate how they connect with this doctoral thesis.

- Alberto Gonzalez Pérez, FPU funded by the "Ministerio Español de Educación, Cultura y Deporte" (grant reference FPU17/03832).

This predoctoral project can be considered a continuation of this thesis, with special emphasis on the technological developments added to the platform 
such as advanced analytics services to generate new insights and actionable information from geospatial data gathered from patients.

- Águeda Gómez Cambronero, FPI funded by the "Consejeria Valenciana de Educación, Investigación, Cultura y Deporte" (grant reference ACIF/2019/798).

This predoctoral project investigates the notion of serious games applied to mental health, taking advantage of the information obtained through the Symptoms platform, and paying special attention on usability and adaptive user interfaces of the mobile applications generated by the Symptoms platform. 


\section{2 \\ Smartphone apps for the treat- ment of mental disorders: a sys- tematic review.}

Don't adventures ever have an end? I suppose not. Someone else always has to carry on on the story.

Bilbo Baggins.

Smartphone apps are an increasingly popular means for providing psychological interventions to patients suffering from a mental disorder. In line with this popularity, there is a need to analyse and summarize the state of the art, both from a psychological and technical perspective. This systematic review focuses on the use of smartphones for psychological interventions. We aim to: (i) analyze the evolution of research over time as well as the covered mental disorders; (ii) study the characteristics of assessments that were performed; (iii) map the use of advanced technical features, such as sensors, and novel software features, such as personalization and social media; and (iv) provide an overview of developed smartphone apps per mental disorder.

The PRISMA guidelines for systematic reviews were followed. We performed searches in Scopus, Web of Science, APA PsycNET and MEDLINE, covering a period of over five years (2013+). We included papers that describe the use of smartphone apps to deliver psychological interventions for known mental disorders. We formed multidisciplinary teams, comprising experts in psychology and ICT, to select and classify articles based on psychological and technical features. 111 articles met the inclusion criteria. We observed an increasing interest in 
this field. More and more studies are focusing on clinical effects in addition to (only) usability/UX, but Randomly Controlled Trials (RCT) are still a small minority $(16,2 \%)$. Around $70 \%$ of the papers focus on 6 mental disorders: depression, anxiety, trauma and stressor-related, substance-related and addiction, schizophrenia spectrum and other psychotic disorders, or a combination of disorders. More than half of known mental disorders are not or very scarcely $(<3 \%)$ represented. Whereas interventions are leveraging the improved modalities (screen, sound) and interactivity of smartphones, their truly novel capabilities, such as sensors, alternative delivery paradigms and analytical methods, are only sparingly exploited.

We found an overall increase in smartphone-based interventions over time. Most research targets disorders with high prevalence, i.e., depressive $(19,8 \%)$ and anxiety disorders $(12,6 \%)$. Hence, there is a need for designing interventions that focus on disorders with high severity as well, such as personality disorders, which are currently only scarcely found. The majority of assessments evaluated usability/UX, and we found only a limited number of RCT. We contend that, to improve the robustness and trustworthiness of assessments, an increasingly systematic focus is needed as to effect on clinical symptomatology. Regarding technical aspects, most studies rather conservatively transfer traditional ICT interventions to smartphones. We argue that more innovative use of their novel capabilities is needed to fully realize promising treatments, such as Ecological Momentary Interventions, as they require context-awareness, though the use of sensors and analytics, to determine the need for interventions at times that patients need them most.

\subsection{Introduction}

The popularity of smartphones has skyrocketed over the last decade, with different sources estimating around $35 \%$ of people owning a smartphone worldwide and even much higher penetration rates in developed countries (ranging from $76 \%$ in the UK to $95 \%$ in South Korea) $[19,20]$. Smartphones are increasingly becoming the most common mobile phone, even in emerging economies [20]. Smartphones

distinguish themselves from their predecessors, which were mainly used for calling and sending text messages, along several dimensions: (a) improved modality (screen, sound), interaction and computational resources, which support sophisticated software applications called (mobile) apps; (b) built-in mobile sensors, which 
Chapter 2. Smartphone apps for the treatment of mental disorders: a systematic review.

allow apps to access various measurements, such as the user's current position, motion, ambient light and sound; and (c) connectivity hardware (WiFi, Bluetooth), which allows virtually ubiquitous Internet connections, and connections to nearby wireless hardware (e.g., headsets, physiological sensors). A plethora of mobile apps has become available, conveniently installable from so-called app stores, which leverage these novel capabilities to varying degrees and address a wide range of personal, entertainment and business needs. In 2017, respectively 2.8 and 2.2 million apps were available from the Google Play and Apple App store, which were downloaded a total of 178.1 billion times [35].

In health [36] and mental health [21], researchers quickly realized the potential of mobile apps-with systematic reviews appearing as early as 2011 [21]. Whereas traditional tele-health [37] and cellphone-based [38] approaches rely on SMS, telephone or video calls, smartphones present a versatile, powerful and personalized platform for delivering a holistic set of care tasks. In the literature, these have included patient screening, symptom and disorder assessment, psycho-education, intervention delivery, progress monitoring and relapse prevention, among others [21]. By providing these health tasks via smartphone apps (at least partially), a number of obstacles for mental health care are reduced, such as therapist workload, lack of qualified personnel, geographic barriers and attitudinal barriers to seek treatment. Moreover, new opportunities arise as well, such as providing ecological, in-situ interventions directly to the patient when they are most needed, as determined by the individual's interaction with the device and sensor feedback (e.g., biofeedback, motion) [22-28].

This is a timely evolution, as reported mental health problems are becoming increasingly prevalent. Trautmann et al. [39] estimate that over $50 \%$ of the population of high and middle-income countries suffers from at least one mental disorder in their life, with a significant impact on their quality of life and an overall annual economic cost of US $\$ 2.5$ trillion (2010) and rising. According to the latest US annual survey, there is an estimated 12-month mental disorder prevalence of $18.3 \%$ among adults ( $4.2 \%$ for serious mental illness). Studies on leveraging mobile technology to meet these challenges, i.e., in the form of mobile mental health interventions, have reported promising mental health outcomes [40-42], large acceptance rates by patients [43], and increased sustainability and preservation of treatment effects [44]. Nevertheless, we find that the possibilities of current smartphone technology have only just been tapped, and further research 
is needed to explore them fully [45], as are studies to analyze the empirical effectiveness of these systems $[45,46]$. For driving and steering such future research, there is a continuous need to establish a state of the art, which comprehensively reviews current focal points on psychological (i.e., type of disorder, type of evaluation) and technological factors (smartphone capabilities, technologies and features used). Such a review should include both exploratory research, which investigates technological opportunities, and empirical research, which establishes the empirical evidence for the efficacy of smartphone interventions. Prior mobile mental health reviews have become dated [47-49], while more recent works only consider specific mental disorders [50] for cognitive impairment, [51] for alcohol and substance abuse, [40] for anxiety or technologies [52] for text messaging and [25] for SMS messages), or focus solely on efficacy, usability and feasibility of mHealth interventions [25, 40, 50, 53].

In this chapter, we provide a systematic review, overviewing the recent (5 years) research in smartphone app-based interventions for mental disorders. Specifically, the goal of the review is to analyse and summarize relevant research, with aims to (i) analyse evolution over time and coverage of mental disorders, (ii) study the type of assessment per mental disorder, (iii) map the use of sensors, software features and analytical capabilities of smartphones, and (iv) provide an overview of developed mobile smartphone apps per mental disorder.

\subsection{Methods}

\subsubsection{Search and Study Selection}

This systematic review uses the Preferred Reporting Items for Systematic Reviews and Meta-Analyses (Prisma) [54] as a guideline. We performed an extensive search of four scientific databases, i.e., Scopus, Web of Science (WoS), APA PsycNET and MEDLINE, using queries that combined search terms related to the psychological (e.g., psychology, psychological, mental disorder, intervention) and the technological dimension (e.g., mobile device, smartphone, mhealth) using logical operators. All database-specific queries were semantically equivalent but formulated using the different syntaxes and technical supports of the respective search engines. Keywords and queries can be found in Supplementary materials $A$ and $B$. 
Chapter 2. Smartphone apps for the treatment of mental disorders: a systematic review.

All resulting publications were downloaded and duplicates were removed. All papers were equally divided among four multi-disciplinary groups of two members, each comprising one computer scientist and one psychologist. Publications were initially screened based on the inclusion/exclusion criteria, using title, abstract and keywords. Subsequently, the remaining and inconclusive papers were fully reviewed and their eligibility was again checked using the inclusion/exclusion criteria. Both during initial screening and the more elaborate eligibility checking, both team members processed the group's assigned papers independently and discussed their observations before making a final decision. In case of disagreement, a third reviewer was assigned and a final decision was made collaboratively.

\subsubsection{Inclusion criteria (IC)}

Articles fulfilling all the following criteria are withheld:

- IC1: Full research articles published in an international journal or conference proceedings since 2013 (including), written in English, and with the full text available.

- IC2: Primary research articles, i.e., articles that produce first-hand and original contributions to the research field.

- IC3: Articles explicitly describing the use of a smartphone app for the delivery of (a) psychological intervention(s) for (a) mental disorder(s), whereby:

- Smartphones are being targeted, i.e., at least one smartphone-specific feature is used, thus going beyond regular mobile phone features (e.g., SMS messages, phone calls) and standard content delivery (e.g., nonmobile, generic websites);

- The considered mental disorder(s) are found in the Diagnostic and Statistical manual of Mental Disorders (DSM-5) [55].

- IC4: Articles including

- Empirical data from real patients;

- Empirical data from participants involved in an experiment / test;

- An elaborate, explicit description of the current or envisioned use of smartphones to deliver psychological interventions. 


\subsubsection{Exclusions criteria (EC)}

All sources that do not comply with the inclusion criterion:

- EC1: All research articles published before 2013, not written in English and/or not published as a full paper in an international journal or conference proceedings. This excludes articles published in any other outlet, such as workshops, discussion fora, colloquia, patent descriptions, white papers, and other types of publications, e.g., posters, demo papers, tutorial paper, editorials, extended abstracts.

- EC2: All secondary research articles, i.e., articles that use primary research articles to derive results, e.g., reviews, systematic maps, meta-analysis, synthesis, comments, etc.

- EC3: Any article not explicitly describing the use of smartphones as the primary mode of delivering psychological interventions for mental disorders. This excludes articles addressing non-mental disorders (e.g., cancer) or symptoms (e.g., stress); as well as articles describing the use of other mobile devices (e.g., wearables, smart watches, tablets) or using smartphones only as a regular phone (e.g., SMS messages, phone calls).

- EC4: Any article mentioning a mental disorder as potential use case or as example application, i.e., only superficially describing its application to a mental disorder. This includes philosophical papers, vision papers or papers solely focusing on a particular technical innovation.

\subsubsection{Classification of studies}

All retained studies were classified according to technology- and psychologyrelated dimensions. The technology-related dimensions include:

- Built-In Sensors: accelerometer, gyroscope, GPS, microphone and camera.

- Software Features:

- Prompting: any kind of pro-active prompting to the patient, e.g., reminders, notifications or motivational messages; 
Chapter 2. Smartphone apps for the treatment of mental disorders: a systematic review.

- Health Care Provider Communication: communicate directly with a Health Care Provider through the mobile app;

- Progress: allow patients to monitor their progress throughout the intervention;

- Assessment: capability to (psychologically) assess the patient, including self-assessment (e.g., questionnaire) and automatic assessment (e.g., based on smartphone usage patterns);

- Social: availability of social networking and peer communication, such as forums, chat, messaging and sharing of experiences or information sources;

- Personalization: ability to customize/personalize some aspects of the mobile app according to the patient;

- Learning: any kind of learning material or support presented to the patient;

- In-Situ Use: explicit support for using the mobile app in the patient's natural environment (ecological) so real-time (momentary) interventions can be offered when they are most needed;

- Context-Awareness: capability of detecting the context/environment of the patient, e.g., location, ambient sound, text/call history, etc.;

- Virtual Reality: use of virtual environments as delivery paradigm;

- Augmented Reality: use of augmented environments as delivery paradigm;

- Game: use of games as a delivery paradigm.

- Analytics: use of advanced software algorithms in the mobile app or supporting infrastructure (i.e., server side)-including machine learning, behavioral analysis, activity analysis, spatial analysis.

- App Name: name of the app; otherwise NA.

The psychology-related dimensions include:

- Mental Disorders: the considered mental disorders are taken from the Diagnostic and Statistical Manual of Mental Disorders (DSM-5) [55]. In addition to the well-established diagnosis categories from DSM-5, we also 
considered a "suicidal behavior disorder/non suicidal self-injury" category, as this condition is very well represented in the literature and recognized as a condition for further study in DSM-5 (i.e., likely to be included in future versions). We note that, in cases where the smartphone app focuses on multiple disorders, we distinguish between (a) those specifically focusing on comorbidity, labeling the app as "comorbid disorders", and (b) those delivering treatment(s) for multiple disorders (not targeting their comorbidity), labeling the app as "various disorders".

- Assessment Type: types of assessment include "Effect", "Usability / user experience" (short: "Usability/UX"), "Effect and Usability/UX" and "No Assessment". "Effect" indicates that the authors reported results about the smartphone app's effects on the participants' clinical symptomatology. "Usability and user experience", as defined by ISO 9241-210:2010 (the international standard on ergonomics of human system interaction) [56], indicates that variables such as usability, user acceptance, opinion and satisfaction, feasibility and intention to use, were assessed by the authors. "Effect and Usability/UX" denotes that "Effect" as well as "Usability and UX" were assessed and reported in the paper. Finally, "No Assessment" refers to those cases where no assessment was reported, e.g., only including study protocols or technical descriptions of the smartphone apps.

\section{3 | Results}

In the interest of transparency and reproducibility, we published the resulting data, code, and a description of the runtime environment on GitHub ${ }^{1}$ and archived the work in Zenodo [57]. The GitHub repository includes a literate programming document that combines text, data preprocessing, analysis, and visualisations. The runtime environment is based on Docker and allows readers to easily open an interactive analysis environment in their browser based on Binder ${ }^{2}$.

\footnotetext{
1 https://github.com/cgranell/apps-treatment-mental-disorders

2 https://mybinder.org/v2/gh/cgranell/apps-treatment-mental-disorders/master
} 
Chapter 2. Smartphone apps for the treatment of mental disorders: a systematic review.

\subsubsection{Study inclusion}

Figure 2.1 shows the results of the systematic review processes according to the PRISMA data flow chart. During the Identification phase, we identified 7933 studies from the four different online sources (Scopus, WoS, APA PsycNET, MEDLINE), which we reduced to 3616 after removing duplicates. After the Screening phase, i.e., based on title, abstract and keywords, we retained 310 articles. The Eligibility assessment, i.e., based on the full paper, led to a final set of 111 papers. More details can be found in Figure 2.1.

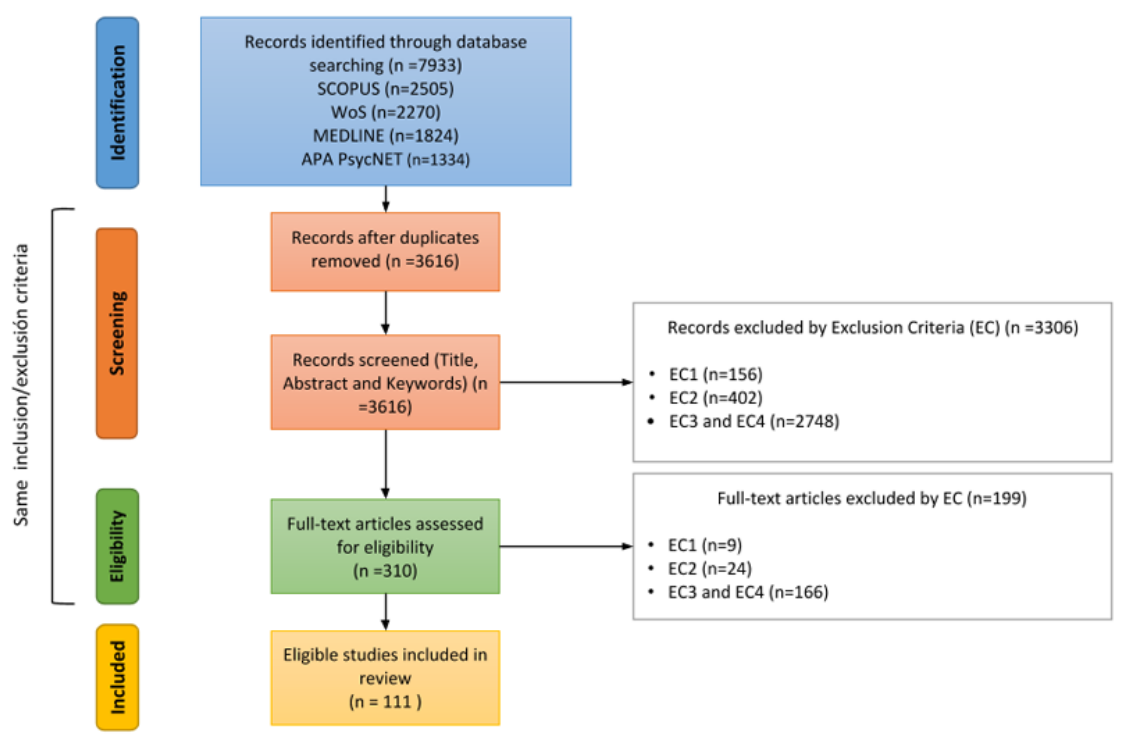

Figure 2.1: PRISMA flow diagram for the systematic review.

\subsubsection{Evolution of research and assessment}

Figure 2.2 (top) shows the temporal distribution of research in the last 5 years, along with their reported assessment type. Overall, we observe a positive evolution of the amount of research over time, steadily increasing from only few (7) articles in 2013 to a relatively large amount (38) in 2017. From this trend, we may infer an overall increased interest in using smartphones for psychological interventions. 
The majority of articles, i.e., 79 out of $111(71.2 \%)$, reported some kind of assessment, whereas 32 articles (28.8\%) reported no assessment at all. As to type of assessment, we notice that only $18(16.2 \%)$ of all studies focus specifically on the effect of intervention on clinical symptomatology (i.e., assessment category "effect"), 34 (30.6\%) report a mix of effect and usability/UX assessment, and $24.3 \%$ focus solely on usability/UX. Looking at the temporal trend, we observe a relative increase of studies performing an assessment specifically on effect, while maintaining the relative amount of mixed and usability/UX assessments roughly at a status quo (Figure 2.2 (down)). Indeed, the 2017-2018 bars show that, in contrast to previous years, the proportion of studies focusing specifically on effect matches the proportion of other types of assessments. Although it is too early to speak of a pattern, we find it a promising sign that this topic is starting to reach sufficient maturity to warrant more research that focuses in particular on effect-which is of primary importance in clinical psychology-in addition to dispersing attention to the combination of effect and usability/UX studies.

Nevertheless, caution needs to be taken when interpreting these results. Orthogonal to the general type of assessment, one should also consider other characteristics of the assessment-i.e., whether it features a Randomized Controlled Trial (RCT) over a long timeframe, or a pilot RCT; or supplies less empirically rigorous results, such as qualitative studies, feasibility studies, case studies (e.g., $\mathrm{n}$ of 1 clinical trials) or usability studies. We observe that, from all studies with an assessment, only a small minority of articles (18 out of $79-22.8 \%$ ) perform an RCT assessment ( 8 on effect, 8 on effect and usability/UX, and 2 on usability/UX only), and only a handful of papers (5 papers, $6.3 \%$ ) perform a pilot RCT; 2 on effect, 3 on effect and usability/UX. (This breakdown is not shown in Figure 2.2.) When looking at the temporal evolution of RCT studies, we see an even spread of effect and usability/UX evaluations over time ( 1 in every year, except 2016 that counts 3 ). For effect studies however, the majority happened in the last years (2 in 2015; 5 in 2017, 1 in 2018). This seems to confirm our earlier observation on the maturing of this research topic, which is not only reflected by an increase in attention to assessing effect, but also an increase of solid, longer term empirical studies (RCTs) focusing on effect. 
Chapter 2. Smartphone apps for the treatment of mental disorders: a systematic review.
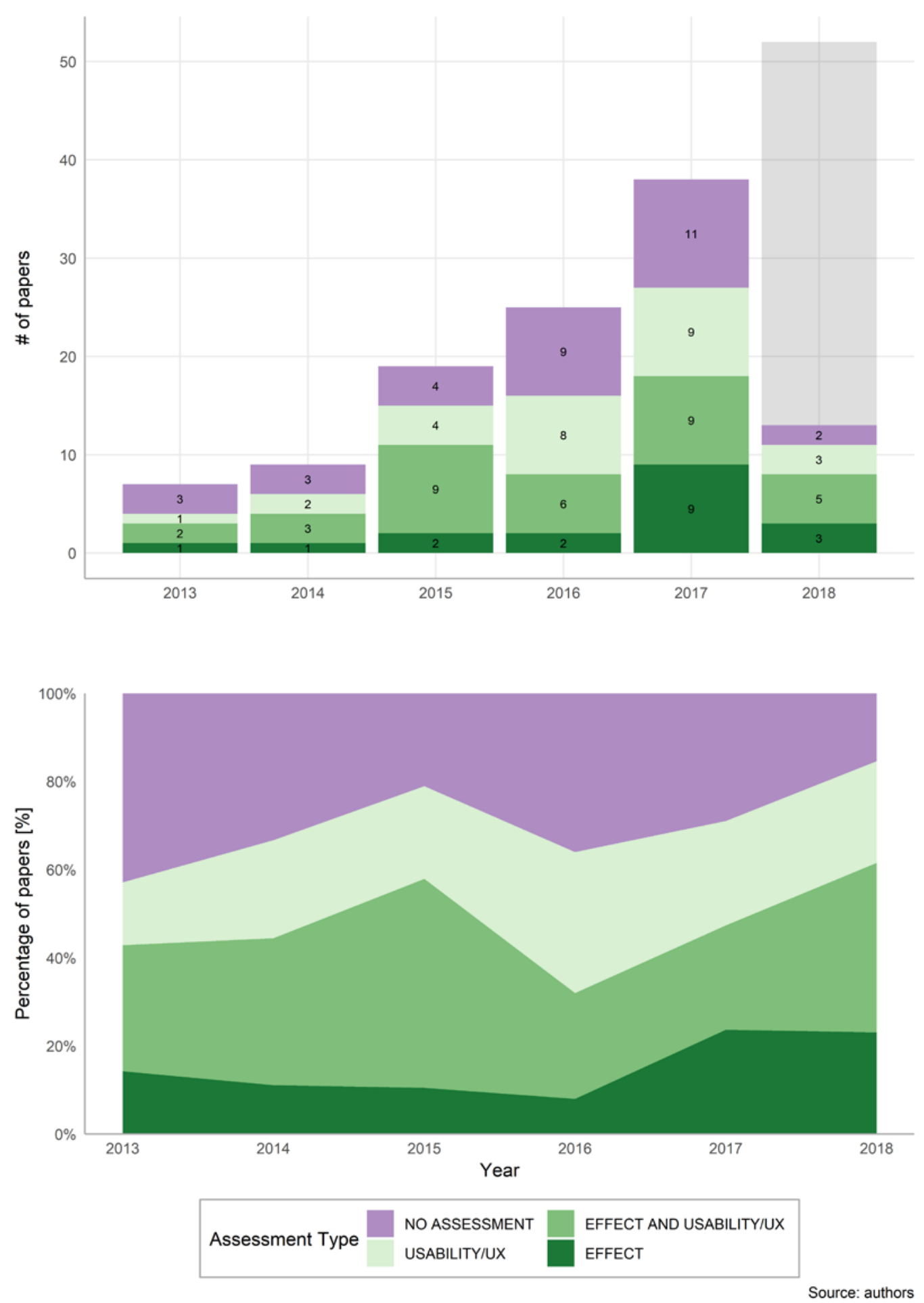

Figure 2.2: Temporal trend of amount of articles published and assessment type. The grey bar in the top figure denotes the estimated number of papers in 2018 (three months covered). 


\subsubsection{Mental disorders addressed}

Figure 2.3 shows the number of studies per mental disorder, ranked in ascending order and sub-categorized according to the type of assessment. The most commonly addressed mental disorders include Depressive disorders (22 papers, $19.8 \%$ ), followed by Anxiety disorders (14 papers, 12.6\%), Various disorders (13 papers, 11.7\%), Trauma and stressor-related disorders (13 papers, 11.7\%), Substance-related and addictive disorders (10 papers, 9\%), and Schizophrenia spectrum and other psychotic disorders (8 papers, 7.2\%). Note that the category of Various disorders includes apps for multiples disorders, where serious mental illness, depressive and anxiety disorders are most represented. Collectively, these top 6 mental disorders account for $72.1 \%$ of all studies included in the search. In the intermediate zone, Suicidal behavior disorder/non-suicidal self-injury (7 papers, $6.3 \%$ ), Comorbid disorders (6 papers, $5.4 \%$ ), and Bipolar and related disorders (5 papers, $4.5 \%$ ) together represent $16.2 \%$ of all studies. We will point out that the majority of papers on comorbid disorders were related to a dual pathology, with a coexistence of psychological disorders with the abuse of substances. Only one case in this category was focused on Neurodevelopmental Disorders and Elimination Disorders. Several mental disorders are only marginally represented in literature: Personality disorders (1 paper, 0.9\%), Obsessive-Compulsive and related disorders (3 papers, 2.7\%), Sleep-wake disorders (3 papers, 2.7\%), Feeding and eating disorders (3 papers, 2.7\%) and Neurodevelopmental disorders (3 papers, $2.7 \%$ ). For the following mental disorders listed in DSM-5, no research at all has been reported (not shown in Figure 2.3: Dissociative Disorders; Somatic Symptom and Related Disorders; Sexual Dysfunctions; Gender Dysphoria; Disruptive, Impulse-Control and Conduct Disorders; Neurocognitive Disorders; Paraphilic Disorders; Other Mental Disorders; Medication-Induced Movement Disorders and Other Adverse Effects of Medication; Other Conditions That May Be a Focus of Clinical Attention.

Finally, Figure 2.4 shows the temporal trend of the top 6 mental disorders targeted by studies over the years. Overall, we observe that the relative ranking of the top 6 mental disorders is maintained. Further, in line with prior observations, we note an increasing amount of published articles for each mental disorder over time. Exceptions include Anxiety disorders, where the amount of articles published remains relatively stable over time; and Various disorders, which' third 
Chapter 2. Smartphone apps for the treatment of mental disorders: a systematic review.

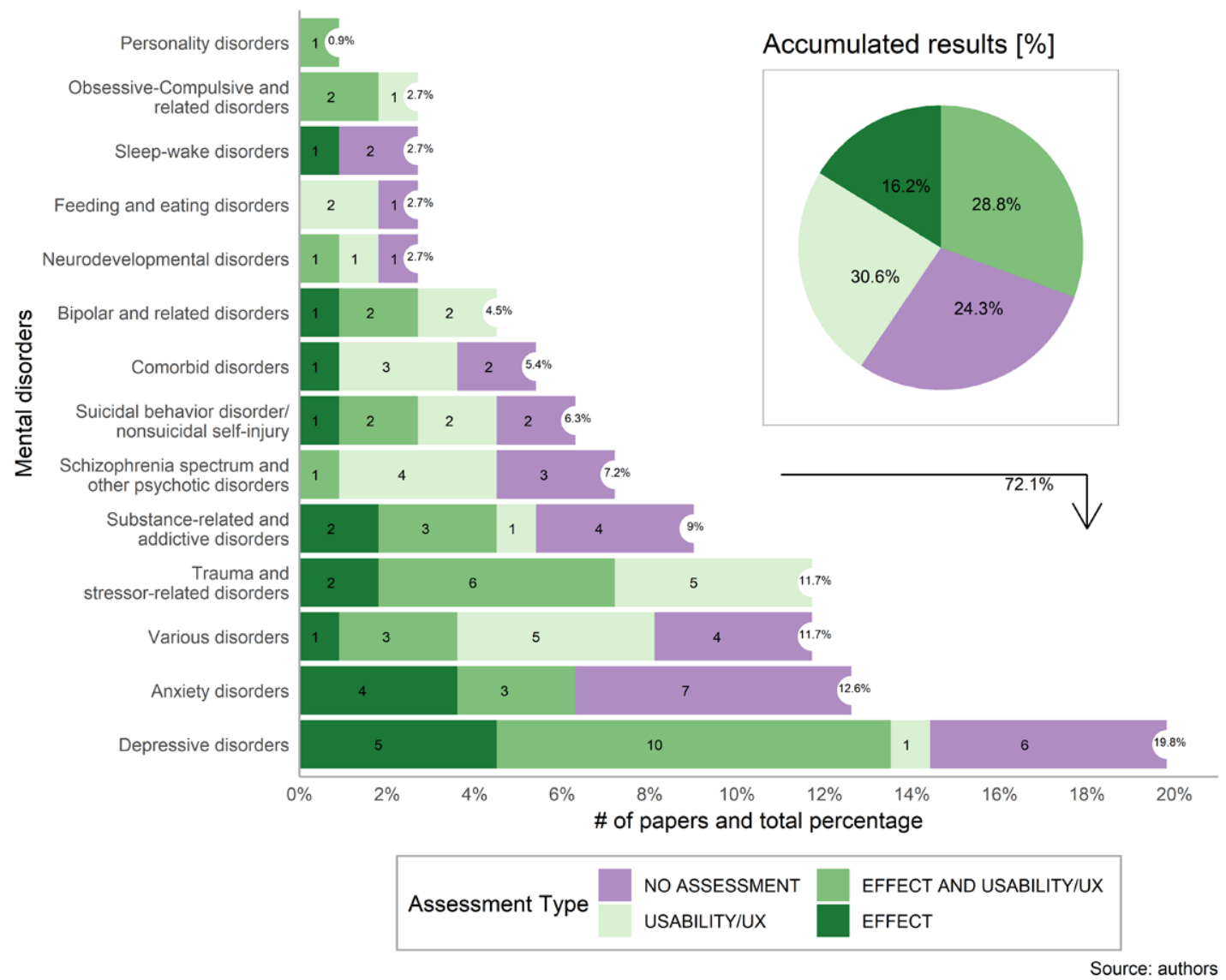

Figure 2.3: Distribution of articles per mental disorder, categorized according to assessment type. The embedded pie chart shows the proportional distribution of types of assessment over all mental disorders.

rank is mostly caused by an outlier in amount of articles (8) published in 2017. We further note that the most addressed disorder, Depressive disorders, has consistently received most research in the last four years (i.e., since 2015). Trauma and stressor-related disorders, the fourth most addressed disorder, has gained relatively more attention in the last three full years of the review (i.e., 2015-2017), ranking second over that timeframe. Finally, notice that relevant research on Substance-related and addictive disorders only started in 2015. 


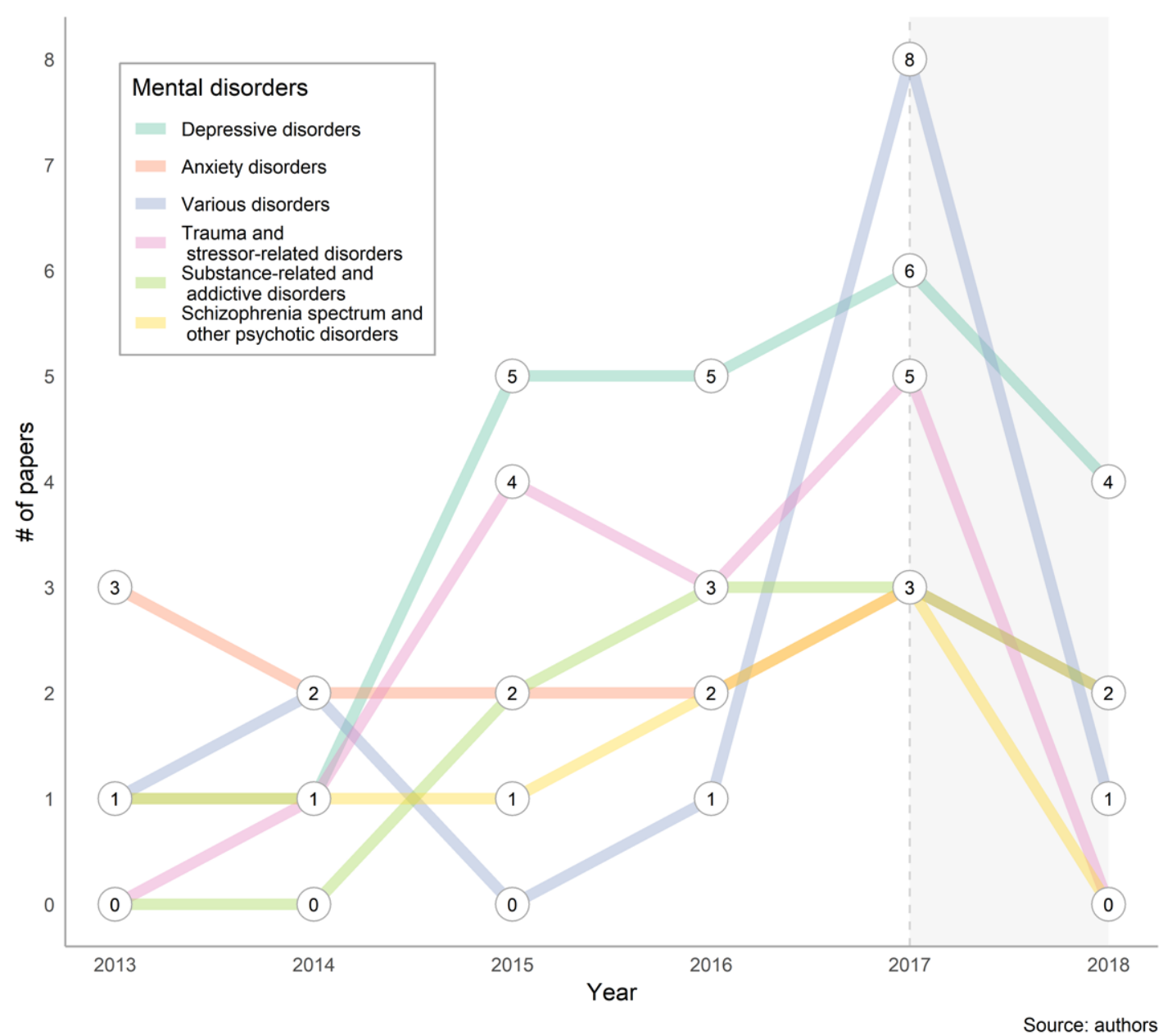

Figure 2.4: Distribution of articles published for the top 6 mental disorders over time.

\subsubsection{Relation between assessment type and mental disorder}

As observed in Figure 2.3, the proportional amount of (only) effect assessments is generally greater for mental disorders that are more addressed in the literature. For the top 6, in decreasing order, we discern respectively 22.7\% (Depressive disorder, 5 papers), 28.6\% (Anxiety disorder, 4 papers), 7.7\% (Various disorders, 1 paper, an outlier), 15.4\% (Trauma and stressor-related disorder, 2 papers), 20\% (Substance-related and addictive disorder, 2 papers) and 0\% (Schizophrenia spectrum and other psychotic disorders, 0 papers) of studies towards effect. On the other hand, for less addressed disorders (outside of the top 6), only 1 or no 
Chapter 2. Smartphone apps for the treatment of mental disorders: a systematic review.

effect assessments were performed. This seems indicate a proposition that the research community, as the body of research is growing for a particular mental disorder, gives more importance to assessing the effect of interventions on clinical symptomatology.

Regarding other types of assessment, no clear patterns can be observed and we fall back to individual observations. Remarkable are the high amount of combined assessments for Depressive disorders (45.5\%) and Trauma and stressorrelated disorders (46.2\%); the low amount of pure usability/UX assessments for Depressive disorders (4.5\%) and Anxiety disorders (0\%); and the high amount of articles without assessment for Anxiety disorders (50\%) which contrasts the high amount of effect assessments.

\subsubsection{Concrete studies per mental disorder and app name}

For the benefit of the research community, Table 2.1 lists, per mental disorder, the concrete studies (by app name, when available) targeting the mental disorder. As can be observed, independent of the mental disorder, most studies utilize a custom-made app which is not reused in other studies. On the one hand, this implies that there exists a wide variety of apps, even for the same mental disorder; on the other hand, only few apps (shown in italics in Table 2.1) have benefited from multiple studies. Remarkably, for Trauma and stressor-related disorders, several apps were reused in multiple studies, and two apps were each reused in four studies. Highlighting some interesting reused apps, Koroko-App (Depressive disorders) is an example app that was rigorously tested both for effect and usability/UX: a study protocol was published, followed by two RCTs assessing effect and usability/UX issues. Other apps, such as PTSD coach and Simply Yoga (Trauma and stressor-related disorders), combine RCTs with other type(s) of assessments for effect and usability/UX. Some apps such as the Blue Ice app (Suicidal behavior disorders/non suicidal self-injury) published assessments using non-RCT designs. 

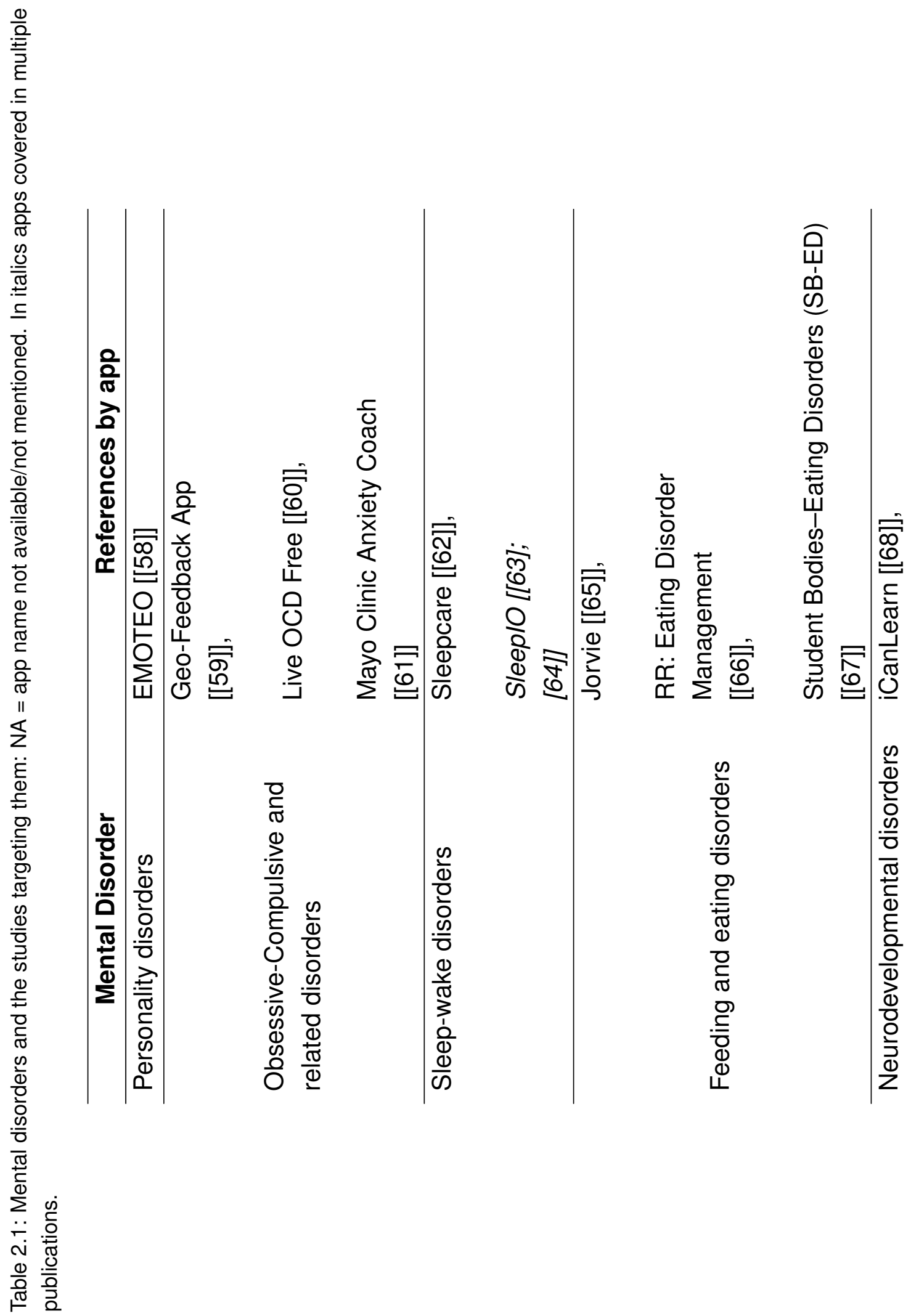
Chapter 2. Smartphone apps for the treatment of mental disorders: a systematic review.

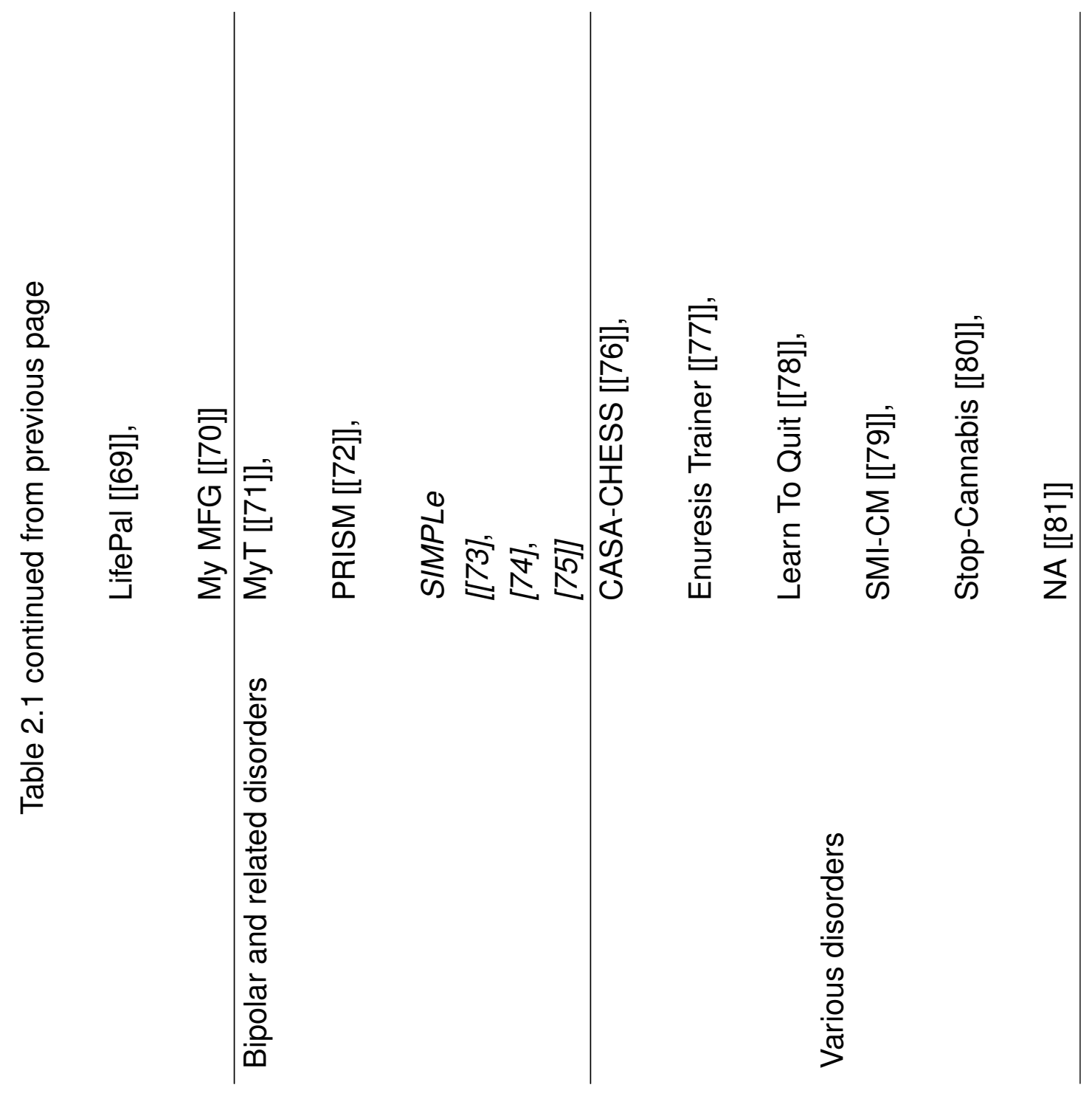




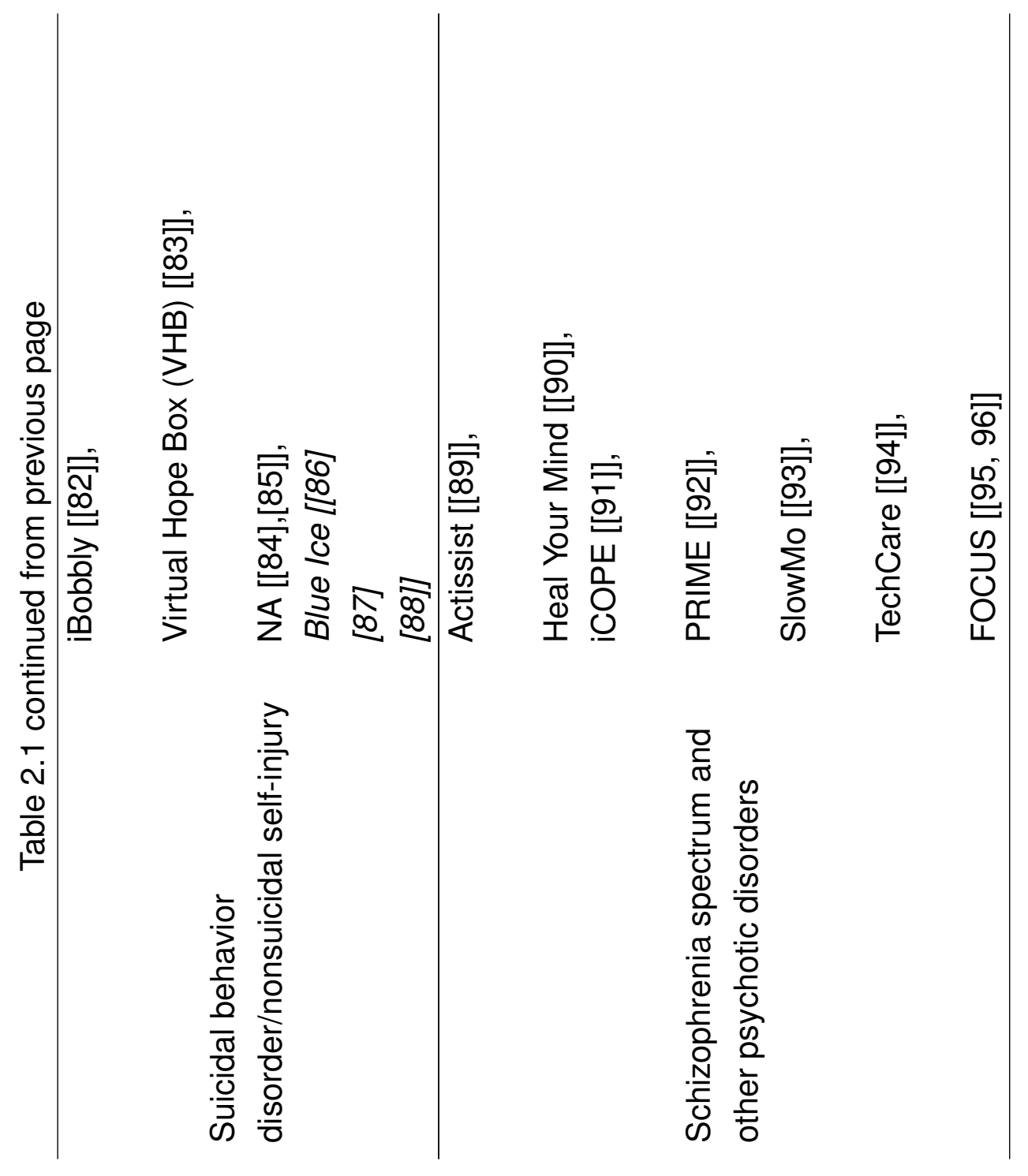


Chapter 2. Smartphone apps for the treatment of mental disorders: a systematic review.

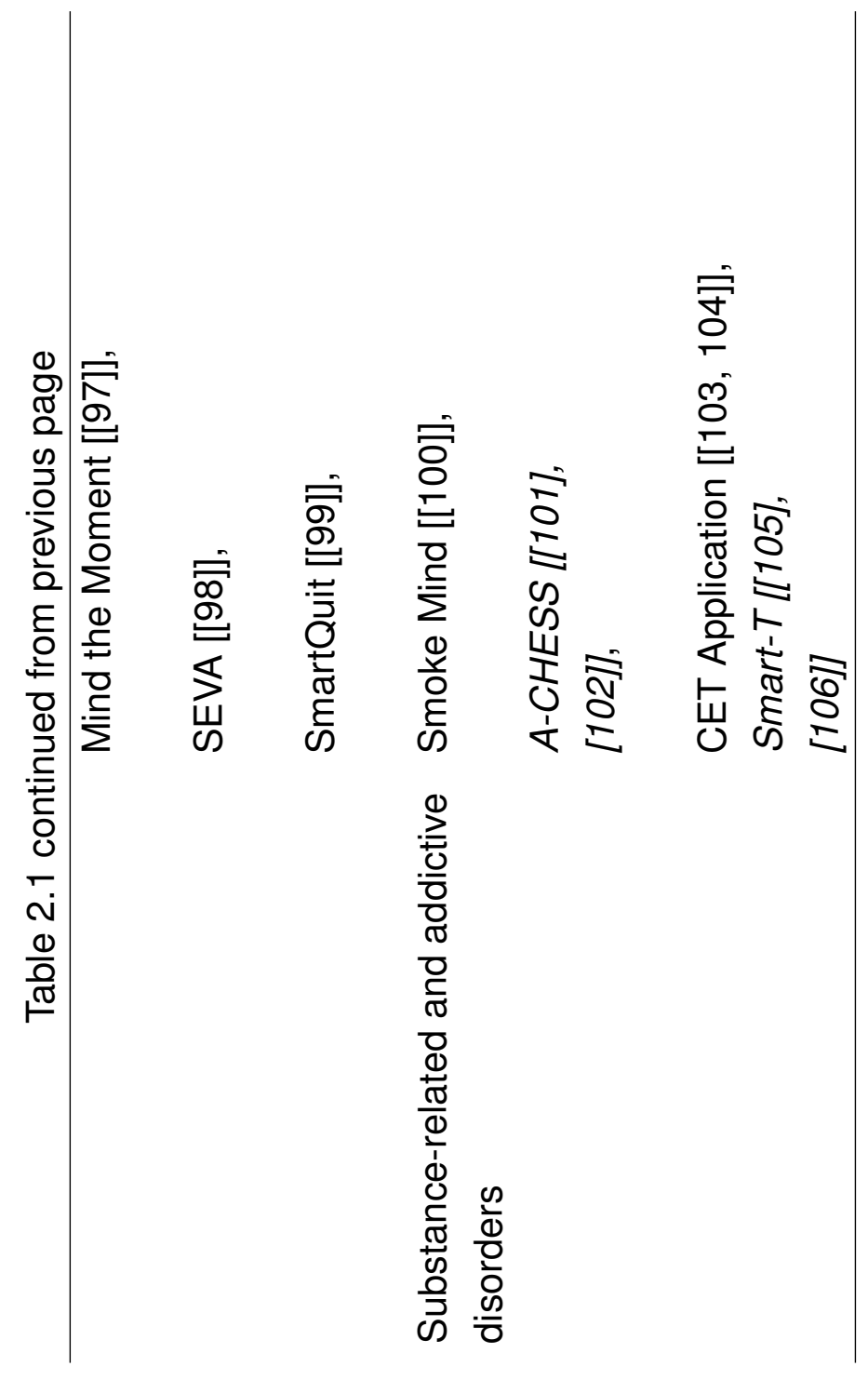




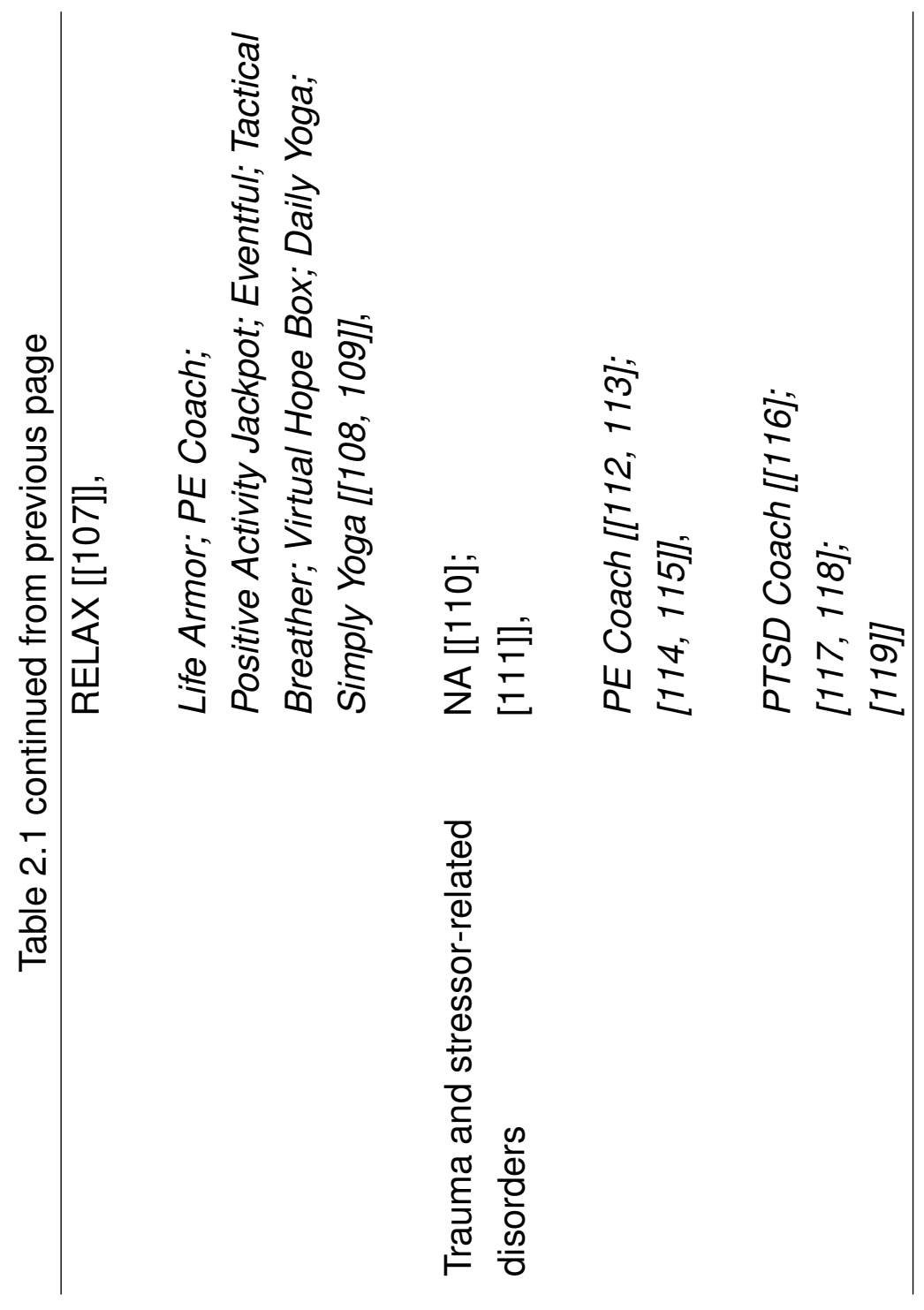


Chapter 2. Smartphone apps for the treatment of mental disorders: a systematic review.

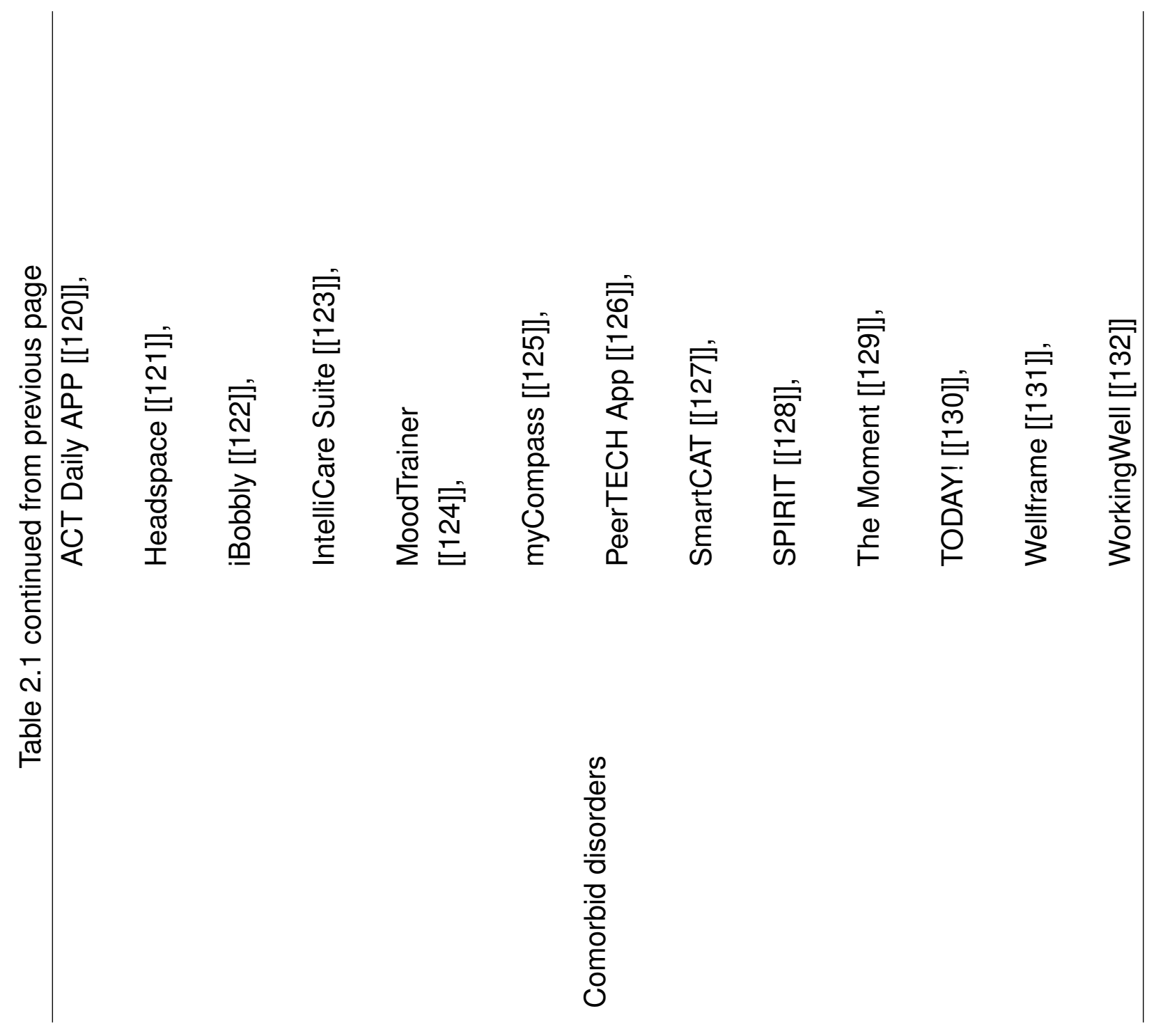




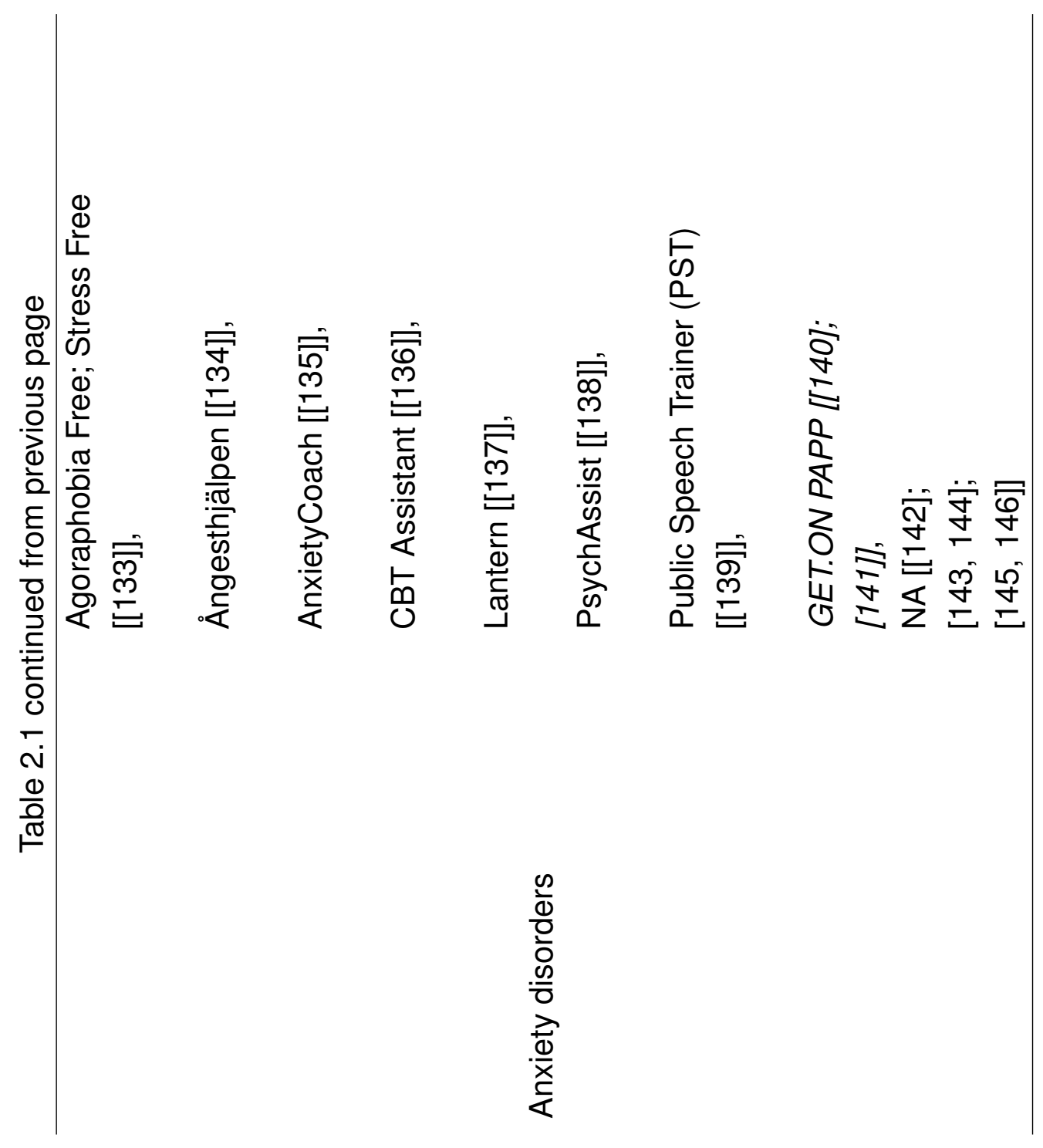


Chapter 2. Smartphone apps for the treatment of mental disorders: a systematic review.

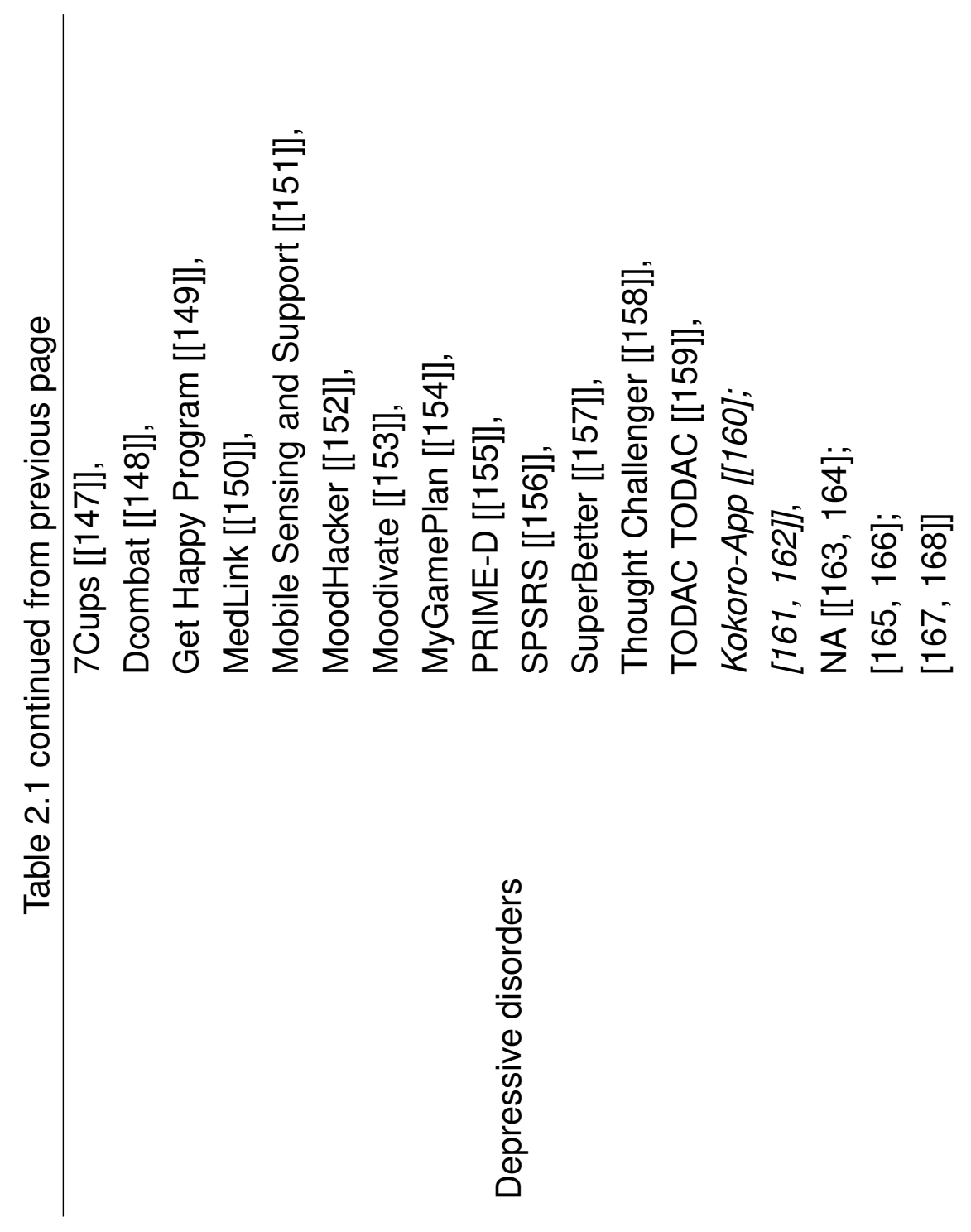




\subsubsection{Relation between mental disorder and technical features}

Figure 2.5 plots the technology-related dimensions, namely software features implemented by the studied apps (in orange), the utilized built-in sensors (in green) and analytics (in blue), versus the type of mental disorders. In doing so, the figure overviews to which extent, and for which disorder(s), the state of the art is leveraging smartphone capabilities, both hardware- (built-in sensors) and software-related (intervention-specific features, communication ability, social features, software delivery paradigm, advanced analyses). Within each technologyrelated dimension ( $\mathrm{X}$ axis), features are ranked by their decreasing popularity over all mental disorders (left to right; total occurrence count of a feature is shown at the top of each column); mental disorders ( $\mathrm{Y}$ axis) are ordered according to decreasing popularity in literature (bottom-up). We note that the most popular mental disorders also accrue the largest bubbles, as their higher (absolute) number of articles tend to cover more varied technical dimensions. Therefore, larger bubbles tend to be concentrated at the bottom of the graph.

Looking at the software features dimension, the top 7 software features are covered by almost all mental disorders, i.e., the top 11 of mental disorders (grey rectangle $7 \times 11$, Figure 2.5 ). These features are mostly related to interventionspecific features, such as learning (ranked 1), progress (ranked 2), personalization (ranked 3), assessment (ranked 4), in-situ use (ranked 7) and communication features, such as prompting (ranked 5) and health care provider communication (ranked 6). In addition, the top 5 mental disorders also cover social media (ranked 8) and context-awareness (ranked 9) (grey rectangle, 9x5, Figure 2.5). Outside of these two rectangles ( $7 \times 11$ and $9 \times 5)$, coverage is more sparse. Regarding delivery paradigm: while games still have a decent coverage ( 8 out of 14 mental disorders covered), virtual and augmented reality are each only covered within 1 mental disorder (respectively, Anxiety disorders and Trauma and stressor-related disorders).

As to utilization of built-in sensors, we observe a much lower and more dispersed coverage without clear patterns. While we did find coverage for each sensor, there is no mental disorder that covers all sensors. Remarkable is the lack of coverage for the microphone (ranked 1) in the top 2 mental disorders (Depressive and Anxiety disorders) and conversely, its high coverage in Trauma and stressorrelated disorders (6). At the other end of the spectrum, we discern low coverage 
Chapter 2. Smartphone apps for the treatment of mental disorders: a systematic review.

for accelerometer (4 articles) and gyroscope (1 article). Finally, we observe very poor support for analytics: only 10 articles support some type of advanced analytics. Horizontally, only Depression disorder (1st ranked disorder) covers all analytics minimally (Machine Learning twice, all others once). Vertically, only Machine Learning and Activity Analysis received coverage by multiple disorders (respectively 5 and 2 disorders). 


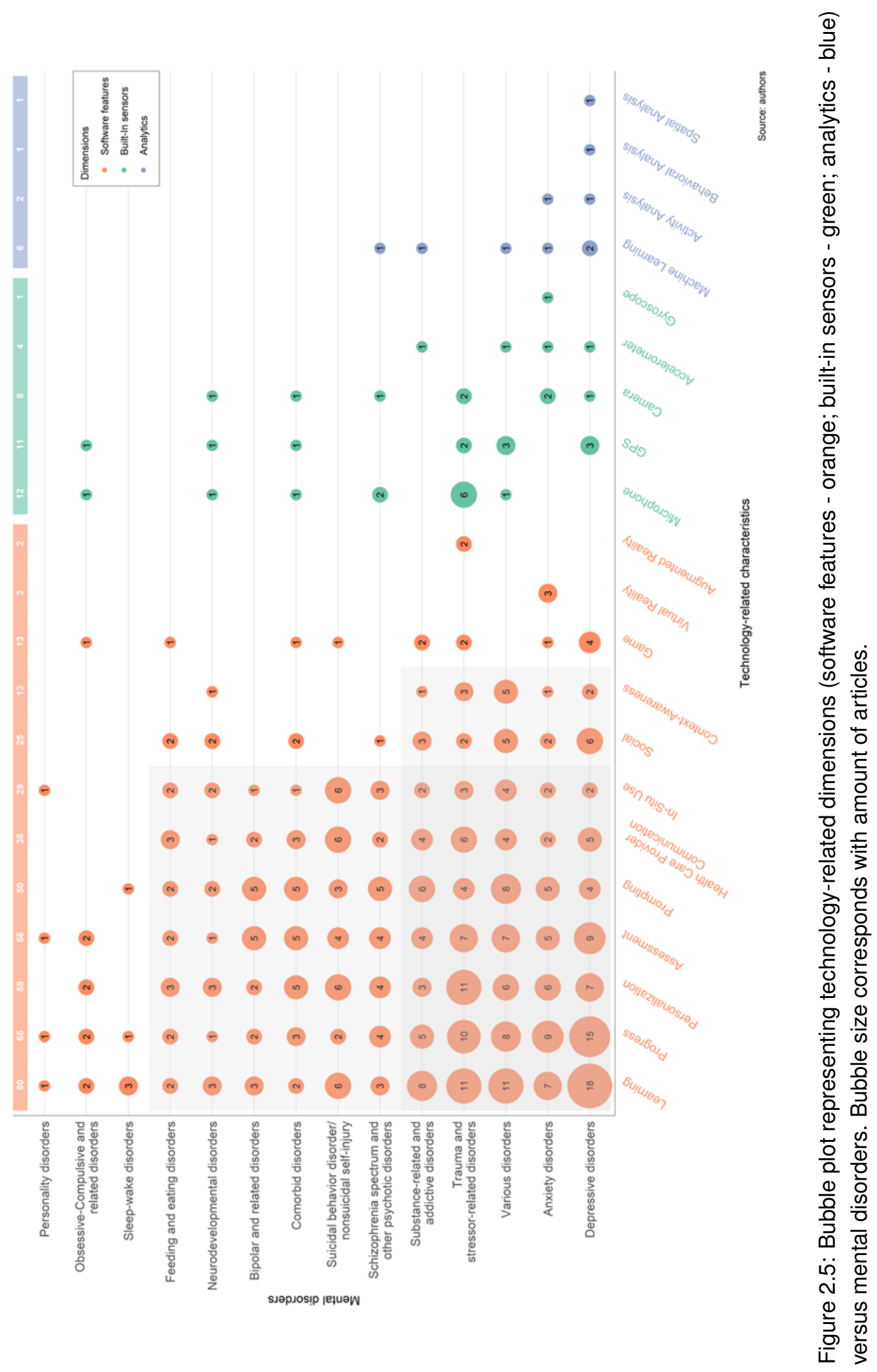


Chapter 2. Smartphone apps for the treatment of mental disorders: a systematic review.

\subsection{Discussion}

\subsubsection{Principal Results}

In general, we observe a growing interest in utilizing smartphone apps for delivering psychological treatments. This is a highly promising trend as such apps can complement therapist-led psychological treatments and hence increase their efficacy. When delegating (a portion of) psychological treatment to smartphonebased interventions, the need for face-to-face sessions and manual follow-up is decreased; which in turn lowers costs and reduces waiting lists in the public health system. According to the mental health workforce breakdown (by WHO region), there are only 4.6 psychologists per 100000 inhabitants/population in Europe [5]. For Spain, studies have reported wait times of more than 45 days before the first psychological assistance by a clinical psychologist or psychiatrist [169], and a frequency of face-to-face sessions of around once a month [6]. Moreover, by offering smartphone-based Ecological Momentary Interventions (EMI), patients can access psychological care when and where they need it most, in their natural environment and daily routines [170-172]. Below, we discuss our concrete observations on the coverage of mental disorders, use of technical features, as well as the assessment types of research studies.

\subsubsection{Coverage of mental disorders}

To an extent, we observe that the coverage of mental disorders in the relevant state of the art is in line with their real-world prevalence. This holds in particular for depressive and anxiety disorders, commonly called emotional disorders, since these affect the most people worldwide and are the most covered by the literature. Other ICT technologies for delivering psychological treatments, such as Internet and web-based programs, are also mostly focused on depressive and anxiety disorders [173, 174]. Depressive and anxiety disorders reduce a patient's psychosocial functioning and quality of life $[175,176]$, and are associated with important personal, social and economic repercussions [177, 178]. The lifetime prevalence of depressive and anxiety disorders have been estimated at $16.6 \%$ $[179,180]$ and $28.8 \%$, respectively. At the same time however, the literature is heavily focused on only a small number of disorders-with six mental disorders 
accounting for ca. $72 \%$ of the encountered articles. Indeed, more than half of the categories of mental disorders listed in DSM-5 (15) are fully excluded or very scarcely represented $(<3 \%)$. Although typically less prevalent, these mental disorders, such as personality disorders, often have a higher severity and hence greater impact on patients' lives. For instance, borderline personality disorder affects $2-6 \%$ of the population $[181,182]$ and its mortality rate by suicide is one of the highest in the world among people with psychiatric disorders [183]. While it would be an interesting exercise to determine the underlying factor(s) behind this distribution of mental disorders in the literature, we consider this beyond the scope of our paper.

\subsubsection{Coverage of technical dimensions}

When looking at technical dimensions, more "traditional" software features (as indicated by the $7 \times 11$ vertical rectangle in Figure 2.5) are much more utilized than the novel sensing or analytical capabilities of smartphones. Indeed, one may argue that this top 7, which involve intervention-specific features (learning, progress, personalization, assessment, in-situ use) and communication features (prompting, Health Care Provider communication), do not offer a significant advancement over the prior state of the art. Many works leveraging mobile phones previously supported learning by displaying psychoeducational content $[74,75,120]$, receiving tips/reminders via SMS [70, 126], using (bidirectional) SMS communication to perform (in-situ) assessment [126], or telephone calls to Health Care Providers $[86,112,123]$. Notwithstanding, even this rather conservative transition to smartphones has led to interventions out of reach for classic mobile phones. Exploiting larger screen resolutions and multimedia capabilities, one finds examples of multimodal learning materials using both audio and video guides [133, 167], using pictures [83, 86-88, 117, 129], audio [83, 87, 88, 117], music [86-88] and video $[83,88,156]$. Leveraging the improved connectivity and ubiquity of smartphones, some authors offer access to entire online libraries of learning materials [127, 167]; others utilize in-app prompting as intervention techniques, e.g., sending reminders to use the app [87, 103, 104, 118, 159], motivational messages [70] or messages from the therapist $[90,163]$. Exploiting the improved interactivity of smartphones, we find interactive quizzes for training skills and improved learning [78, 127], assessments for panic attacks [144], suicidal intentions [122], symptoms 
Chapter 2. Smartphone apps for the treatment of mental disorders: a systematic review.

of various disorders $[71,105,106]$ and communication with therapists $[58,74,91$, $144]$ or other users $[92,108,109]$ through message/chat. Aside from being better supported by smartphone capabilities, many of these psychological smartphone interventions are available at the touch of a button, instead of relying on receipt of SMS or phone calls.

That said, most works still only scratch the surface of advanced smartphone capabilities. This is particularly apparent in the lack of context-awareness, i.e., leveraging sensors to detect and react upon the current state of patients and their environments. We argue that such context-awareness is a key ingredient of EMI. Indeed, whereas EMI are meant to proactively issue suitable therapeutic interventions at the right time and place, we found that most EMI works consider smartphones merely as a tool for manually accessing interventions, or receiving predefined interventions at set time intervals, at any moment and place. Despite this lack of context-awareness, it is promising that we did find a few studies that utilize sensors to improve assessment., such as using GPS for connecting locations to patient mental states [129]. Outside the scope of this review, we also found a few relevant studies: e.g., using microphones to record the frequency and duration of face-to-face conversations [184]; camera for tracking affect based on facial expressions [185]; and accelerometer (among others) to assess sleep quality [184]. We also found a very limited number of smartphone-based works leveraging external sensors for recording physiological parameters and hence illustrating context-awareness: measuring heart rate for detecting physiological arousal in the context of anger management [107], for instance. We note that there exist studies using wearable devices without the use of a smartphone $[186,187]$. In our opinion, studies that progressively use internal and/or external smartphone sensors are a useful step towards realizing the full potential of EMI, where relevant events are detected through analysis of sensor readings, and acted upon by triggering suitable, personalized interventions when they are needed. The general hesitance towards context-aware EMI could be explained by the lack of validated computerized psychological models (i.e., analytics) for assessing mental states based on physiological and environment parameters; as well as the need to combine technologically advanced solutions (i.e., use of sensors, context-awareness).

Through their improved modalities (screen, sound), interactivity and computational resources, smartphones enable novel intervention delivery paradigms, 
including virtual and augmented reality, as well as comprehensive gamification elements. Similarly however, these have found only very limited coverage in literature. We found a few individual studies, for example, utilizing a mobile virtual reality (VR) system to help patients coping with agoraphobia by guiding an avatar through real-life simulations in a game-based setting [133]. Beyond exposure-type therapies, Repetto et al. (2013) [143] utilize VR techniques to cope with generalized anxiety disorders, leveraging bio-feedback to regulate features of the virtual world (e.g., current heart rate). As before, the seeming lack of non-traditional intervention methods may be caused by the lack of validated psychological models for supplying evidence-based VR, augmented reality or gamification elements and/or the technical difficulty of novel delivery paradigms.

Given the lack of studies on these topics, we believe that there lies a huge potential for future research in utilizing technologically advanced solutions (i.e., use of sensors, context-awareness, alternative delivery paradigms) to deliver smartphone-based psychological interventions tailored to the patient's current health context.

\subsubsection{Assessment types in research studies}

We observe that most current studies involve an evaluation of usability/UX. Usability factors have been widely recognized as key factor to enhance the acceptability of ICT tools. Based on the technology acceptance model, authors have suggested that the intention to use a product in the future is strongly correlated with its ease of use $[183,188]$. Hence, initial efforts to research and ensure the usability of new ICT tools are essential. At the same time, we observe an increasing amount of research that focuses on the effect of smartphone interventions on clinical symptomatology. Moreover, the relative amount of effect studies increases as the mental disorder receives more coverage in the literature. We consider this a promising trend, as it points towards the maturing of the research field by focusing on the (long-term) clinical effects of the intervention, in addition to the design, development and (usability) evaluation cycle of the smartphone intervention.

Nevertheless, we observe that RCTs, which are considered the "gold standard" of experiment design in the field of mental health (and medicine in general), are only minimally represented in the literature ( $16.2 \%$ of articles overall). In order to draw robust and trustworthy conclusions on the clinical efficacy of smartphone apps, 
Chapter 2. Smartphone apps for the treatment of mental disorders: a systematic review.

more long-term RCT studies will be needed (e.g., to better measure the effects of attrition). Similarly, we observe a distinct lack of cross-validation studies, with only a few apps having been studied by multiple articles. One could note that this phenomenon is correlated with the lack of robust long-term studies on smartphone interventions-a single, multi-year study would warrant multiple articles for a single smartphone intervention on study protocol, usability evaluation, and effect studies at multiple intervals. Particularly when utilizing novel technological features, robust assessment studies are needed to validate their potential for psychological interventions and encourage further research in the field. A stronger cooperation between research groups could increase the resources needed for such long-term psychological intervention studies.

\subsubsection{Recommendations for the research community}

In line with our findings, we propose 4 recommendations for the research community to further develop and advance the field of smartphone-based psychological interventions:

- Attention for low-covered disorders: the gravity of research lies with highly prevalent mental disorders (ca. 72\% of research in the top 6 mental disorders), thereby virtually ignoring more than half of the mental DSM-5 recognized disorders. More research is needed in low-covered disorders, especially those with high severity, such as bipolar and related disorders or even less represented disorders such as personality disorders.

- Attention for advanced technical and software-based solutions: many smartphonebased psychological interventions merely translate classical and eHealth solution to the smartphone as a delivery platform. Although these interventions benefit from the improved capabilities of smartphones, such as larger screen sizes, interactivity and multimedia, the interventions do not fully exploit their capabilities. Concretely, the use of sensors and corresponding context-awareness, particularly to promote EMls, the exploration of alternative delivery paradigms such as games, virtual or augmented reality, and more advanced analytical methods, are scarcely investigated. We call upon the research community to explore beyond traditional strategies, towards leveraging advanced technological features to improve the efficacy of psychological interventions. 
- Multi-disciplinary approaches: to fully exploit smartphones' capabilities as a pervasive, ubiquitously connected, sensor-packed computing platform to deliver innovative, real-time and in-situ psychological interventions, both the domain knowledge of psychologists as well as the technical expertise of computer scientists is needed. Hence, we call for multi-disciplinary collaborations as to not let technical difficulties, or lack of psychological knowledge on mental disorders, hinder advances and novelties in the field.

- Validation towards effect: even though we uncovered a slight increase in effect validations and RCT-based effect assessments during the last years, they are still under-represented (particularly RCTs). Hence, there exists a need to robustly validate smartphone-based psychological treatments for effect. Especially when utilizing advanced technical features (e.g., contextawareness, analytics, alternative delivery paradigms), effect validation may bestow trust and spark further research in such novel type of interventions. We call upon the research community to augment efforts in robust effect assessment, in order to allow transfer of research into practice.

\subsubsection{Strengths and Limitations}

The main strength and novelty of this work is to explore and summarize, under the prism of a wide range of technical characteristics, the current state of the art in smartphone-based interventions for mental disorders. We hereby provide a broad overview of the field: (a) covering the full spectrum of mental disorders as classified in the latest version of DSM, rather than focusing on a specific mental illness as done in previous studies; and (b) exposing technical features used to realize smartphone-based treatments. Consequently, this contribution is highly innovative as a synergetic study targeting mental health research and recent developments in mobile sensing and computing. Further strengths of this study include the use of four different bibliographic sources for a comprehensive coverage of the research and literature, and the methodological process based on pairs of multidisciplinary researchers for the selection, validation and classification of literature.

As any systematic study, search term specification may lack other relevant terms not considered by the authors, whereas searches only cover literature written in English. Therefore, there always exists a risk to not fully identify all relevant studies. Classification of studies may also be prone to error. To reduce this risk, we used 
Chapter 2. Smartphone apps for the treatment of mental disorders: a systematic review.

pairs of researchers from different disciplines with a requirement of inter-rater agreement. Finally, another limitation of our study was its partial coverage in 2018. Notwithstanding, we note that we covered five full years (2013-2017), which coincides with the period of rapid expansion of relevant research compared to previous years (2008-2012). While the era of smartphones began in 2008, the developments during the first few years were virtually only testimonials.

\subsubsection{Conclusions}

We presented a comprehensive systematic review of the state of the art in smartphone-based psychological interventions, with a synergetic focus on psychologyrelated issues, such as mental disorders and type of assessment, as well as technological features, such as software features and device sensors. Our results show a rapid increase over recent years in the number of psychological interventions for various mental disorders using smartphone-based apps. It captures how depressive $(19,2 \%)$ and anxiety disorders $(12,6 \%)$ are primarily covered, in line with their real-world prevalence. Apps covering various disorders $(11,7 \%)$, trauma and stressor-related (11,7\%), substance related and addictive $(11,7 \%)$ and schizophrenia spectrum and other psychotic disorders $(7,2 \%)$ complete the top 6 , which together accounts for $72,1 \%$ of coverage. This implies the need for further development and research of smartphone interventions for mental disorders that are under- or not represented, particularly those with high severity such as bipolar and related disorders, personality disorders, or dissociative disorders. On the technical side, the review highlights a group of software features related to intervention (learning, progress, personalization, assessment, in-situ use) and communication (prompting, health care provider communication) deployed in smartphone interventions that mostly mimic more traditional mobile phone and eHealth solutions. More innovative use of smartphones' capabilities, such sensing, alternative delivery paradigms, and advanced analytics, are only scarcely present in literature, despite their potential for advancing existing solutions, such as EMls. With regards to the type of assessment, we found that most current studies involve an evaluation of usability/UX, with a slow progression towards effect studies. RCT studies are still a minority in the studies included in this review. 



\title{
Considerations for designing context-aware mobile apps for mental health interventions.
}

\author{
I know many things that only the \\ wise know.
}

Gandalf the grey.

This work identifies major areas of knowledge and proposes a set of relevant dimensions by area that must be taken into account in the design and delivery of context-aware mobile applications for mental health interventions. We argue that much of the related research has focused only on a few dimensions, paying little or no attention to others and, most importantly, to potential relationships between them. Our belief is that the improvement of the effectiveness of mobile interventions to support mental health necessarily implies that developers and therapists comprehensively consider the interaction between the proposed dimensions. Taking as a starting point the three areas of knowledge (Technology, Context and Mental Health), we re-examine each area to identify relevant dimensions, discuss the relationships between them and finally draw a series of considerations.

The resulting considerations can help therapists and developers to devise, design and generate custom mobile applications in a way that increases the motivation and engagement of patients and, therefore, the effectiveness of psychological treatments. 


\section{1 | Introduction}

According to a 2014 WHO systematic review ${ }^{1}$, one fourth (27\%), around 83 million people, of the adult population (aged 18-65) in European Union countries had experienced at least one mental health disorder (e.g., substance use, psychoses, depression, anxiety, eating disorders) in the past year. Other sources cite 165 million Europeans each year have a diagnosed mental health disorder, and more than $50 \%$ of the population in middle- and high-income countries have at least one mental disorder at some point in their lives [2]. Within the spectrum of mental health disorders, depression is most prevalent, affecting almost $5 \%$ of the world's population according to $2017 \mathrm{WHO}$ data [189].

The total estimated number of people living with depression increased by almost $20 \%$ between 2005 and 2015 [3].

Furthermore, there are several indications that mental health disorders can expand in a worrisome way, especially in adolescents. San Diego State University Jean $M$. Twenge described the sudden jumps in mental health disorders since 2012, in comparison to the gentle slopes in past epochs [190]. These findings are also supported by a recent international study investigating the prevalence of psychological disorders in eight developed countries among first-year universitylevel students: one in three students report symptoms of a mental health disorder [191]. Among other actions, research and innovative developments to alleviate these problems are required to improve public health and reduce the associated socio-economic impact of the increasing level of mental health disorders [192].

Human health, including mental health, "is part of an interdependent multifaceted system" [193, pp. 1023]. This is not a new idea. Indeed, other influential factors have been taken into account in the prevalence of mental health issues. For example, environmental health researchers have long recognised the importance of geographic context for understanding the effects of different environmental factors such as noise level and air pollution on mental health [29, 30]. Urban practitioners and planners have also studied how the configuration and features of the built environment along with the morphology of cities may influence citizens' health [31-34]. Essentially, a large body of research has focused on responding from different perspectives regarding how the natural and built environment affect

1 WHO-Europe, Data and resources. Retrieved from https://bit.ly/2BZe2ZK on 5 December 2018 
Chapter 3. Considerations for designing context-aware mobile apps for mental health interventions.

people's health, i.e. to understand the degree and ways in which the features and manifestations of the environment influence public health and aspects of our lifestyle in cities [194, 195]. Computer scientists are recently gaining importance in the field as the increasing democratisation and deployment of mobile computing, mobile devices and sensing technologies [196] provide new opportunities to deliver mobile mental health care [197-199] thus improving quality of life [200]. Various authors $[199,201,202]$ have suggested that interventions with mobile phones designed to support evidence-based treatments could even reduce barriers to access and increase participation in treatment. The rapid and wide adoption of these new developments and technological innovations is leading to a new stream of research that explores the prevention of mental health disorders through mobile-based interventions [203].

We recognise the value and vital contributions of past research to the discipline. Our belief is that most studies consider a limited view, usually restricted to considering pairs of dimensions such as the built environment and mental health, or mobile technologies and mental health. Ferrás et al. [204] move forwards and add the geography and location to the equation, starting a dialogue about the potential benefits of bringing geography (and location), mobile technology and mental health together [204]. In this context, recent research works confirm the assumption that considering distinct aspects may lead to better tools for mental health interventions. For example, Palmius et al. [205] collected location data from mobile phones and assess the level and regularity of geographic movement of patients. By adding the location in their approach for delivering interventions, their results suggest a strong correlation between geographic movements and patients with depression in bipolar disorder. Saeb et al. [206] follow the same strategy of passively collecting location data from mobile phones to estimate an individual's most visited places and locations. In a subsequent study, the same authors demonstrate that location data may be a reliable predictor of depressive symptom severity [207].

These examples suggest that a greater variability of the dimensions and factors involved in mental health interventions can have a positive effect in the design and impact of location-based mobile tools for such interventions. We believe that taking a holistic view to study the interdependent and multifaceted field of mental health leads to greater levels of contextualisation for personalised interventions. This chapter expands this idea by conducting an exploratory exercise in the variability of influential dimensions and their interrelations for context-aware mobile apps in 
mental health interventions. Our long-term research goal is the design and development of a software platform to inform psychologists and therapists for the rapid design and creation of mobile apps that are well suited to mental health disorders. This is aligned with a recent call to implement improved interventions through the use of technological advances (among which include mobile interventions), which was identified as one of the six research priorities in mental health for Europe [192].

In this chapter, we perform a preliminary analysis to identify interrelated dimensions which could be regarded as conceptual building blocks, similar to the set of software patterns devised by Buschmann et al. [208] and Gamma et al. [209]. In these seminal works, the concept of software pattern refers to a generic solution to a specific class of design issues, i.e. a well-defined strategy is applied to solve similar problems. Consider for example, a software tool that's aimed to search content from multiple social media services (e.g. Twitter, YouTube, Wikipedia, Open Street Maps), alleviating the user from understanding the specific data formats and data access protocols to query and retrieve data from each service. This issue has been long studied from the perspective of software engineering; Buschmann et al. [208] proposed the software pattern Broker that can be easily understood as an intermediate component (middle-ware) between users and the different systems. Users only interact with this middle-ware component instead of with an arbitrary number of distinct systems. Many examples have adopted this pattern in varied application domains $[210,211]$ as a generic solution to reduce complexity and improve scalability and interoperability.

This chapter reflects on the foundation for the establishment of patterns -i.e. the identification of well-defined strategies, each applicable to diverse scenarios and situations-, for the design of mobile applications for mental health interventions. We identify the key dimensions and their relationships of three areas of knowledge, namely Technology, Context and Mental Health. The following research questions drive the remainder of this chapter (The acronym numbering refers to the research question that tries to solve the specific chapter: RQ2, plus the specific question posed in this work: RQ2.1, etc):

- RQ2.1: What are the relevant dimensions that sustain or describe each area of knowledge (Technology, Context and Mental Health)?

- RQ2.2: How do the dimensions that belong to different areas of knowledge 
Chapter 3. Considerations for designing context-aware mobile apps for mental health interventions.

relate to each other?

- RQ2.3: Which interrelated dimensions may best inform users for designing context-aware mobile apps to support mental health interventions?

\subsection{Methods}

The present study combines various qualitative research techniques together, following the tenets of the grounded theory method [212, 213]. In short, the grounded theory is "the attempt to derive theories from an analysis of the patterns, themes, and common categories discovered in observational data" [214, p. 307]. In this study, the "discovered theory" in terms of expected findings is comparable to the set of considerations to inform designers and therapists in the process of designing context-aware mobile applications for mental health interventions. Our choice of research methods to obtain this knowledge from empirical data looks directly at the general theory of scientific knowledge [215], which is composed of facts, concepts, principles, theories and laws.

Our focus in this chapter is limited to the chain "facts-concepts-principles", which allows us to move from the observational data (facts) to the establishment of meaningful relationships among facts and concepts (principles).

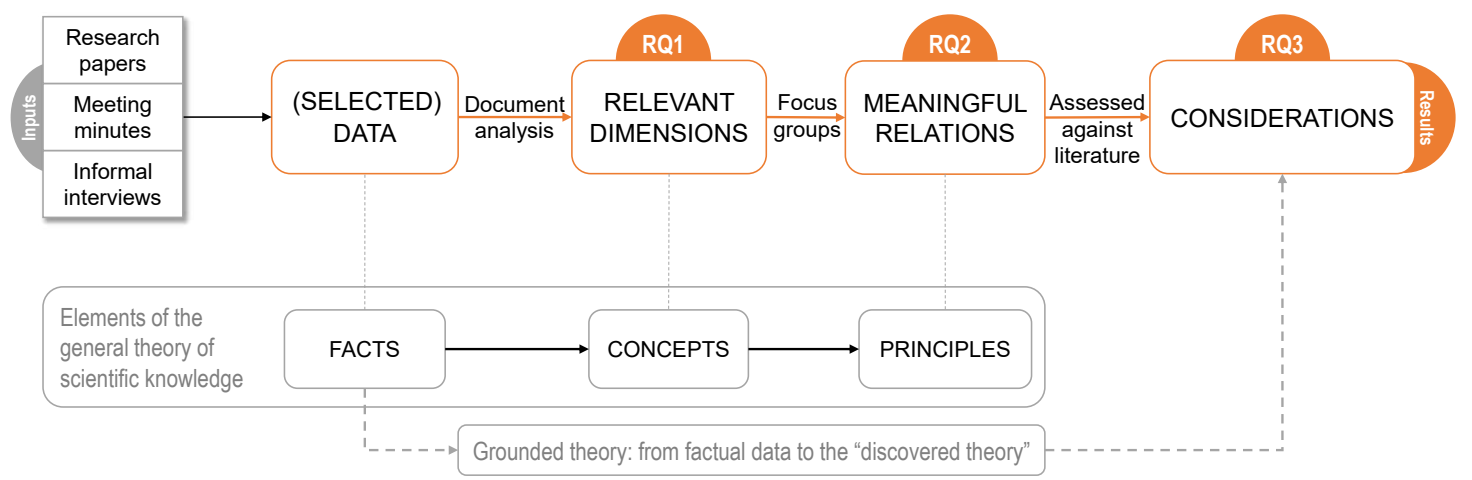

Figure 3.1: Qualitative research methods in relation to the RQs and their correspondence with the elements of the general theory of scientific knowledge [215] and the basics of the grounded theory method [212, 213].

Figure 3.1 illustrates the qualitative methods approach connected to the research questions (Section 3.1), and how they correspond with the tenets of the general theory of scientific knowledge and the grounded theory. The extraction of concepts 
(i.e., significant dimensions) from facts is related to RQ2.1 (Section 3.1), while the identification of principles (i.e., meaningful relations between dimensions) and their assessment against existing literature refer to RQ2.2 (Section 3.3.2) and RQ2.3 (Section 3.4), respectively. The combination of various qualitative research methods for the collection and selection of observational data allows us to gather a good understanding and a practical reality of the potential use, benefits and impact of mobile applications for mental health interventions.

\subsubsection{Document analysis}

To answer RQ2.1, we used the document analysis method as a qualitative research method. Bowen [216] defines it as "a systematic procedure for reviewing or evaluating documents" [216, pp. 27], to find, select, make sense of and synthesise data in the analysed documents. Extracted data are then "organised into major themes, categories and case examples" [216, pp. 28]. The advantages of the document analysis in our exploration are twofold. First, it allowed us to corroborate observational data across sources to reduce the potential bias of using a single information source or research method for data collection [217, 218]. We used various sources to obtain a more complete picture of the research topic, which allowed us to validate similar concepts from different sources to triangulate the data findings. Second, content analysis [214] is commonly used in combination with document analysis method to organise key data and information extracted from the documents into categories (i.e. coding) for subsequent data processing and reexamination. This is the main reason behind the identification of relevant dimensions (RQ2.1) and the re-interpretation of the relations between them (RQ2.2).

Evaluated documents in the document analysis method are not restricted to published research papers (journal articles and conference papers), but other forms of documents are also relevant, such as books, doctoral theses, minutes of meetings, institutional reports and other public documents and records. Therefore, document analysis is not a research method for data collection, but it pursues data selection [216]. The selected documents are interpreted to make sense of a particular research topic. Therefore, it is worth noting that document analysis can be used in combination with other research methods that focus on data collection like systematic reviews, but is not a type of review. In this study, we did not perform 
Chapter 3. Considerations for designing context-aware mobile apps for mental health interventions.

a review. Rather, we use document analysis as a qualitative research method to evaluate a range of selected documents to extract and interpret a set of design considerations. Next, we describe the procedure for selecting the main forms of documents.

Research papers were an important source of documents in our exploration. We selected widely-used citation databases such as Scopus and Web of Science. We first compiled a list of key terms for each area of knowledge by screening previous review papers and concept papers from the related literature. Based on internal discussion among the authors and the exchange of information with psychologists through regular meetings (below), we selected the final set of research papers (listed in Table 3.3) as input for the document analysis method.

Regular meetings between the authors (computer scientists and technologists) and a group of psychologists from the LABPSITEC, a research group specialised in applying technology to mental health disorders, were a pragmatic way to establish a continuous dialogue and an effective communication channel between the two disciplines. Informal interviews were also conducted at the same time as the meetings. Nevertheless, only some meetings were followed with interviews of individual therapists. The interviews combined an "interview guided approach" and a "standardised open-ended interview" [219, pp. 438-442]), which means that a few questions were previously predefined according to the planned agenda of the meeting, but the remainder of the questions were based on the content of the conversation. In general, the meetings and informal interviews allowed us to collect essential or distinctive features that would otherwise not be able to be collected via other traditional means such as on-line surveys and questionnaires. For example, barriers, problems and fears perceived by therapists of using mobile technology in treatments for depressive symptoms. Equally important was to capture, through these interviews, the expectations of the therapists and future ideas about the application of mobile technology in their mental health interventions [220].

Ten meetings were conducted between June 2017 and July 2018 to get a better understanding of how geospatial concepts and related technology could make a real, social impact for technology-driven interventions in mental health. The number of participants per meeting was approximately 4-5 people, with half being psychologists and the other half being computer scientists. Minutes of the meetings were summarised and centralised in a public Github repository (https://github.com/cgranell/labpsitec-ideas). 
In summary, we interpret and evaluate various forms of documents through the document analysis method, such as research papers, meeting minutes, and observational and qualitative data collected from informal interviews. These are the main types of documents selected for the document analysis method (Figure 3.1).

\subsubsection{Focus groups}

To answer RQ2.2, we ran two focus groups [214]. The first focus group was aimed at exploring the relationship between context and mental health. Given the multidisciplinary objective of the focus group, the background of the participants was intentionally related to distinct disciplines and areas of knowledge such as Context, Mental Health, Education and Technology. The second focus group was aimed at drawing conclusions about the use of technology in mental health interventions. In this case, the required profile of participants were more restricted than in the first focus group: health professionals with a certain level of technological expertise was the dominant profile. LABPSITEC researchers helped us with the recruitment of eligible participants for the focus groups.

The final participants of the focus groups were emailed to briefly explain the topics to be discussed ahead of the meeting. On the same day of the meeting they all signed an informed consent form to ensure ethical and privacy considerations of the participants. Both focus groups were recorded. We took notes during the focus group, complemented afterwards by a content analysis of the recorded video to extract the main highlights of the meeting. The two focus groups were organised in two phases. The first phase consisted of a guided discussion where participants were asked key questions and were allowed to comment and discuss each of them for twenty minutes. In the second phase, participants could extend the topic and debate more freely with a longer time frame duration. In both focus groups, participants were encouraged to consider the role of geographic elements and location as drivers of the discussion.

The first focus group engaged participants from multidisciplinary profiles (therapists, psychologists, computer scientists, architects) in a discussion about the relationships between context and mental health. In a first step, twelve professionals, three from each area, were contacted through phone calls and emails. Nine participants $(\mathrm{N}=9)$ were finally recruited. Socio-demographic data of the participants is shown in Table 3.1. The focus group meeting took place 31 January 
Chapter 3. Considerations for designing context-aware mobile apps for mental health interventions.

2017, in the Universitat Jaume I of Castellón (Spain), and lasted two hours. The key questions were: What and how the characteristics of the urban environment can influence the physical activity/mental health; What aspects of games and how they can be regarded as tools for motivation and engagement; How these games can take advantage of the characteristics of the urban environment.

Table 3.1: Sociodemographic data of the participants of the focus group one. BD = Bachelor Degree, CS = Computer Science, GIS = Geographic Information Systems

\begin{tabular}{lllll} 
Participant & Professional profile & $\begin{array}{c}\text { Education } \\
\text { Level }\end{array}$ & Gender & Age \\
\hline Participant 1 & Physical Education & BD & Male & 35 \\
\hline Participant 2 & $\begin{array}{l}\text { CS and } \\
\text { GIS }\end{array}$ & PhD & Male & 40 \\
\hline Participant 3 & Clinical Psychology & PhD & Female & 37 \\
\hline Participant 4 & Psychology and Ergonomics & PhD & Female & 40 \\
\hline Participant 5 & Clinical Psychology & PhD & Female & 44 \\
\hline Participant 6 & Architect & PhD & Male & 45 \\
\hline Participant 7 & Physical Education & BD & Male & 35 \\
\hline Participant 8 & CS and Education & PhD & Female & 51 \\
\hline Participant 9 & CS and Education & PhD & Female & 49
\end{tabular}

The second focus group, which was more specialised in the scope than the first, brought together psychologists and therapists involved directly with mental health interventions to discuss the relationships, benefits, barriers and implications of mobile technology and mental health. We emailed initially twelve people. Finally eight participants $(\mathrm{N}=8)$ were recruited and took part in the focus group meeting. Socio-demographic data of the participants is shown in Table 3.2. The focus group meeting took place 22 January 2018, in the Universitat Jaume I of Castellón (Spain), and lasted one hour. Participants were asked to describe the role of technology in the interventions they have designed and/or actively participated in, as well as to imagine the potential uses of technology that could help answer the following questions. In the case of depression, for example, how many times has a patient left home? How much time has a patient spent away from home? What if a patient has not left home all week? In the agoraphobia interventions, the questions focused on how close a patient has been to the mall (or other crowded 
place); or how much has a patient managed to escape from the comfort or security zone? For gambling, key questions were: has a patient approached a gambling area? How long has a patient been there? How many times has a patient left?

Table 3.2: Sociodemographic data of the participants of the focus group two. The ICTs Knowledge column represents the participant's ability with the use of information and communication technologies. Zero means non ICT knowledge and four denotes an expert level.

\begin{tabular}{lllll} 
Participant & ICT Knowledge & Education Level & Gender & Age \\
\hline Participant 1 & 2 & PhD & Female & 38 \\
\hline Participant 2 & 2 & PhD & Female & 30 \\
\hline Participant 3 & 3 & Bachelor Degree & Female & 35 \\
\hline Participant 4 & 2 & Bachelor Degree & Female & 26 \\
\hline Participant 5 & 2 & Bachelor Degree & Female & 31 \\
\hline Participant 6 & 4 & PhD & Male & 32 \\
\hline Participant 7 & 2 & Bachelor Degree & Male & 23 \\
\hline Participant 8 & 2 & Bachelor Degree & Female & 26
\end{tabular}

\subsubsection{Assessment}

As a final step (the right side of Figure 3.1) and to address RQ2.3 - which interrelated dimensions may best inform users for designing context-aware mobile apps to support mental health interventions-, we evaluated the resulting relationships between the dimensions (outcomes of RQ2.2) with the existing literature. The objective was to evaluate these relationships with the evidence and to summarise them as a set of considerations that can inform therapists in the process of designing context-aware mobile applications for successful mental health interventions.

\section{3 | Results}

\subsubsection{Relevant dimensions of each area of knowledge [RQ2.1]}

In this section we address RQ2.1 - what are the relevant dimensions that sustain or describe each area of knowledge? Technology, mental health and context are the three areas of knowledge in which our exploration is framed (see Figure 3.2). 
Chapter 3. Considerations for designing context-aware mobile apps for mental health interventions.

However, as these areas cover a lot of ground, we reduce them to a set of relevant dimensions. This reduction (not simplification) is necessary to identify the relevant aspects of the areas of knowledge that can make an impact on mental health interventions. A best practice in the document analysis method is to establish the meaning and contribution of the analysed sources to the research topic [216]. To this end, Table 3.3 summarises the main sources and references from the literature used for the re-interpretation of each identified dimension of our exploration.

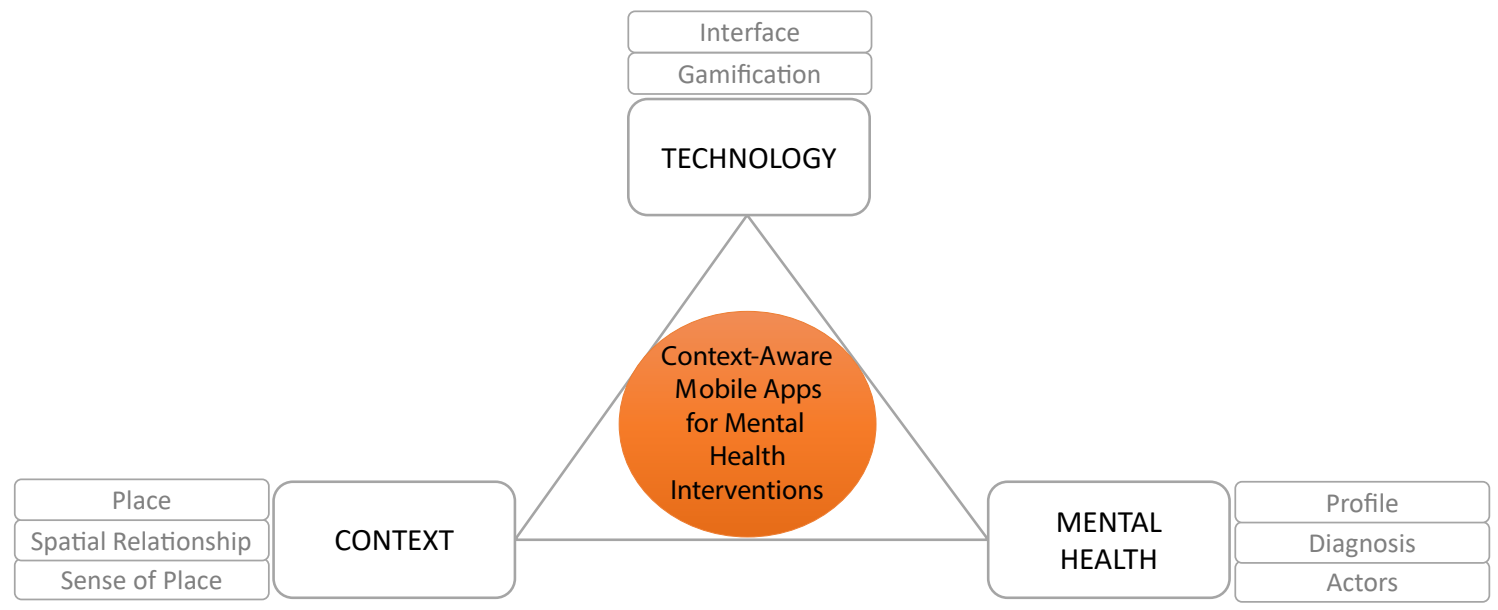

Figure 3.2: Areas of knowledge and the relevant dimensions that influence the design of contextaware mobile apps as mental health intervention.

\section{Technology}

Technology has had a long and successful marriage with psychological interventions for mental health, not only in the creation of new technology-based interventions and treatments, but also to streamline traditional health processes (e.g., sharing health records).

With the arrival and the rapid penetration of the smartphone (2.53 billion people use smartphones in 2018 [19], and the increase in functionality in the past years (e.g., connectivity, processing capacity and memory, detection capabilities), the range of mobile applications for mental health interventions is rapidly expanding [201, 221-223]. If mobile devices, technology and mobile computing have dramatically impacted (mental) health to the point of coining the term m-health [197], the use of video game technology as an innovative way to treat psychological treatments is also a reality [224]. These two ideas framed our exploration to identify 
key dimensions derived from the area of knowledge pertinent to technology.

A quick review of the related literature tells us that the most widely used strategy has been the digitisation of standardised questionnaires previously established in the mental health field in a mobile application, such as the patient health questionnaire (PHQ-9) for depression screening [225]. The momentary and ecological characteristics of these types of intervention are ensured thanks to the ubiquitous and pervasive nature of mobile devices [226]: patients are able to fill in, smoothly integrate during their daily routines, digital questionnaires (or other data collection techniques) at any time, anywhere. Beyond that, the hardware and software possibilities offered by these intelligent devices are, in most cases, underutilised. This suggests that the vast majority of these developments are limited to moving from a traditional way of operating to a digital medium (i.e. digital transformation [227]), but paying little or no attention to the new characteristics of the digital medium [228]. For example, technical characteristics such as built-in sensors, location analysis, compatible operating systems, battery requirements, performance, network needs and communication interfaces are characteristics to be carefully evaluated in order to integrate them into intervention tools for the efficient delivery of content to patients. In this study, we group all of these characteristics under the umbrella of the term "interface". Therefore, interface is one of the relevant dimensions of the technology area.

One of the main problems of mobile interventions is the lack of patient adherence to their treatment. A 2013 study identified engagement techniques as one of the essential features for the success of mobile interventions for mental health [229]. Videogames are a promising technology for improved participation and engagement practices [230]. Indeed, applications of online and offline video games for mental health therapies have been extensively reviewed [224, 231], suggesting that different genres of existing video games could yield great benefits in mental health interventions.

Our focus here is on how game design strategies and game dynamics can be applied to non-game contexts like mental health. This is widely known as gamification [232]. The intention to use gamification in mental health interventions is to exploit its inherent ability to immerse a player (patient) in a story (intervention) to convey a key message and/or achieve a goal. For example, metaphors can help therapists to design an engaging game story for an intervention, such as Spallazzo \& Mariani's [233] location-based mobile game that turned the symptoms 
Chapter 3. Considerations for designing context-aware mobile apps for mental health interventions.

of depression and their consequences into a metaphorical story to improve the learning experience and increase the awareness of healthy people (players) with mental health illnesses. Other examples of gamified interventions for mental health are already being utilised [234]. Thus, it is worth exploring gamification as a relevant dimension related to compliance, engagement, and attractiveness to mitigate drop-out rates for interventions based on mobile devices.

\section{Context}

Several studies have shown that the distribution of the urban environment is an influential factor in the amount of mobility that a population presents. Equally important are the different features of the urban environment, which facilitate or complicate moderate physical activity [31,32] and have effects on mental health [34]. In our previous work, Miralles et al. [235] explored the natural and built environment features that were perceived as the most relevant ones to encourage physical activity. Features like access to public open spaces, availability of bike lanes or presence of drinking fountains appear at the top of the list, in concordance with other studies [236, 237]. The analysis of urban characteristics is an important factor when planning psychological interventions in cities, because people (patients) develop their daily routines and activities in their cities. Urban places are not only important because of physical proximity, but also because of the social and emotional bonds that people acquire in places that are meaningful to them. These two ideas drove our exploration of well-established theories in the fields of Geographic Information Science [238, 239], Human Geography [240] and Environmental Psychology [195], in order to identify the relevant dimensions that relate to the urban context.

Ahlqvist \& Schlieder [241] completed an in-depth analysis in the realm of geogames or location-aware games [242] to align existing theories of core geographic concepts in the GIScience field, namely Kuhn [243], Kuhn \& Ballatore [244], and Janelle \& Goodchild [245], to location-based game patterns such as exploring, collecting objects, co-locality, and spatial structure [246]. The authors propose a unified set of core geographic concepts and compare them to existing spatial game patterns [246], which resulted in two main groups of spatial patterns: locality and proximity. Due to their generality, we focus on them here and reinterpret beyond the geogames domain. Locality means to be in a place but accounting also for 
the topological relationships (e.g. disjoint, meets,) between geographic objects $[247,248]$. For example, the meet relationship means that two spatial regions are touched like the boundary of the countries of Spain and Portugal, and the disjoint refers to separate regions like each island in the Canary Islands. Considering these relationships, locality refers to entering a place, leaving it, or crossing it, all of them seen as triggered events [241]. Since locality implies a formal and informal way of specifying a place, we use place instead of locality because the former is intuitive and widely used in Human Geography and Environmental Psychology. Proximity, the second fundamental spatial pattern, is defined as "the variation of distance to a neighbourhood or location" [241, pp. 9]. We refer to proximity as the distance to a place (e.g. 500 metres) or qualitative appreciation (e.g. close, far), considering it as a type of spatiotemporal association. Lü et al. [249] recently suggested the term relationship as "a general term for spatiotemporal association among geographic elements" [pp. 352]. Built upon the notions of proximity, spatiotemporal association and relationship, we propose spatial relationship as a relevant dimension of context.

Places can evoke feelings and/or lived experiences [250, 251], and are central pieces of human experience with implications for the development of identity, affection and empathy [252-254]. Place can be defined as "a particular space which is covered with meanings and values by the users" [255, pp. 187] and plays a significant role in human behaviour and mental health [195]. For example, the way in which individuals perceive themselves depends on how they feel in their daily places and interact with them [256]. In this sense, the concept of sense of place has been studied for a long time in Environmental Psychology and Human Geography to refer to human experiences, emotions, thoughts, meanings, values and feelings associated with places [250]. Jorgensen \& Stedman [257] define it as the cognitive and behavioural dimensions of the relationship that an individual has towards a specific geographic area. In section 3.3.1, Lü et al. [249] also pointed out the concept of "relationship" and expanded it to cover "other interactive linkage among multiple geographical elements" in addition to spatiotemporal associations such as physical, chemical and biological interactions. Built upon the concepts of sense of place, emotional bonds and places, we propose the sense of place dimension as a type of relationship between people and spatial settings. 
Chapter 3. Considerations for designing context-aware mobile apps for mental health interventions.

Mental Health

Although there are multiple approaches that address mental health, CognitiveBehavioural Therapy (CBT) is one of the most dominant forms of psychotherapy [258]. Within the area of mental health, we intend to offer some reflections on the dimensions that should be considered when carrying out context-aware mobile apps interventions in the CBT approach.

The profile of the patient is key in designing an intervention. Recently, some aspects such as gender or age have been highlighted as important features to defining user preferences in technology-based psychological interventions and, specifically, in mobile applications for health [259, 260]. Given this evidence, the first dimension proposed is the profile of the patient.

Given a profile, it is important to establish the corresponding diagnosis and, consequently, the evidence-based treatments [261]. This may also imply the identification of the commonalities among the emotional disorders supported by the emerging extensions and innovations of the CBT approach, like the unified transdiagnostic protocol [262, 263]. The therapy will have requirements, including technical developments, which may imply great repercussions on cost and time. A close link between the logical therapeutic components for the target disorder and the technical requirements to increase efficiency rates will be thus necessary [222]. Therefore, we take diagnosis as the second key dimension.

It is common for family members, couples or people close to the patients to actively participate in the therapy too [264, 265]. The term actor in software modelling specifies a role played by an external user or entity that interact with a system. Here, we borrow this terminology to refer to actors as therapists and familiar members, who are externals to the intervention but at the same time play a specific role in it. Therefore, it is important to take into account this possible variation of actors or other people in the interaction with the treatment itself, because it can have influence the technical developments that are applied in the intervention [265]. In consequence, the dimension of actor represents key roles besides the patient (e.g. therapists, caregivers, family members, etc) involved in the therapeutic process. In summary, three key dimensions stem from the mental health area: the profile of the primary users or patients, the diagnosis, and the actors, encompassing other roles in the intervention. 
Table 3.3: Summary of selected dimensions grouped by area of knowledge, together with the main sources and references analysed to reinterpret each dimension.

\begin{tabular}{|c|c|c|}
\hline Area & Dimension & Main sources and references \\
\hline \multirow{4}{*}{ Technology } & Interface & BinDhim et al. [225], Rana et al. [196], \\
\hline & & Haggerty [227], and Bohnsack et al. [228] \\
\hline & Gamification & Seaborn \& Fels [266], \\
\hline & & $\begin{array}{l}\text { Nacke \& Deterding [267], Bartle [268], } \\
\text { and Tondello et al. [269]. }\end{array}$ \\
\hline \multirow{10}{*}{ Context } & Place & $\begin{array}{l}\text { Kuhn [243], Kuhn \& Ballatore [244], } \\
\text { Janelle \& Goodchild [245], }\end{array}$ \\
\hline & & $\begin{array}{l}\text { Ahlqvist \& Schlieder [241], } \\
\text { and Sintoris [246]. }\end{array}$ \\
\hline & Spatial & $\begin{array}{l}\text { Ahlqvist \& Schlieder [241], Lü et al. [249], } \\
\text { Randell et al. [247], and }\end{array}$ \\
\hline & Relationship & Egenhofer et al. [248]. \\
\hline & Sense of Place & Stedman [250], Tuan [251], Duff [252], \\
\hline & & Najafi \& Shariff [255], \\
\hline & & Gotham \& Brumley [256], \\
\hline & & Jorgensen \& Stedman [257], and \\
\hline & & Acedo et al. [253]. \\
\hline & Profile & Andone et al. [259], and Zeng et al. [260]. \\
\hline \multirow{5}{*}{ Mental Health } & Diagnosis & Nathan \& Gorman [261], \\
\hline & & Van Ameringen et al. [222], \\
\hline & & Barlow et al. [262], and \\
\hline & & Ellard et al. [263]. \\
\hline & Actors & $\begin{array}{l}\text { Turkington et al. [264], } \\
\text { and Echeburúa et al. [265]. }\end{array}$ \\
\hline
\end{tabular}

\subsubsection{Relations between dimensions [RQ2.2]}

To address RQ2.2 - how do dimensions belonging to distinct areas relate to each other?, we ran two focus groups to explore the potential relationships between 
Chapter 3. Considerations for designing context-aware mobile apps for mental health interventions.

the identified dimensions (Figure 3.3) in the process of designing context-aware mobile apps for mental health interventions. The exploration is organised into sections, each of them covering a couple of areas at the same time, in the same way that the guided discussion was carried out during the focus groups.

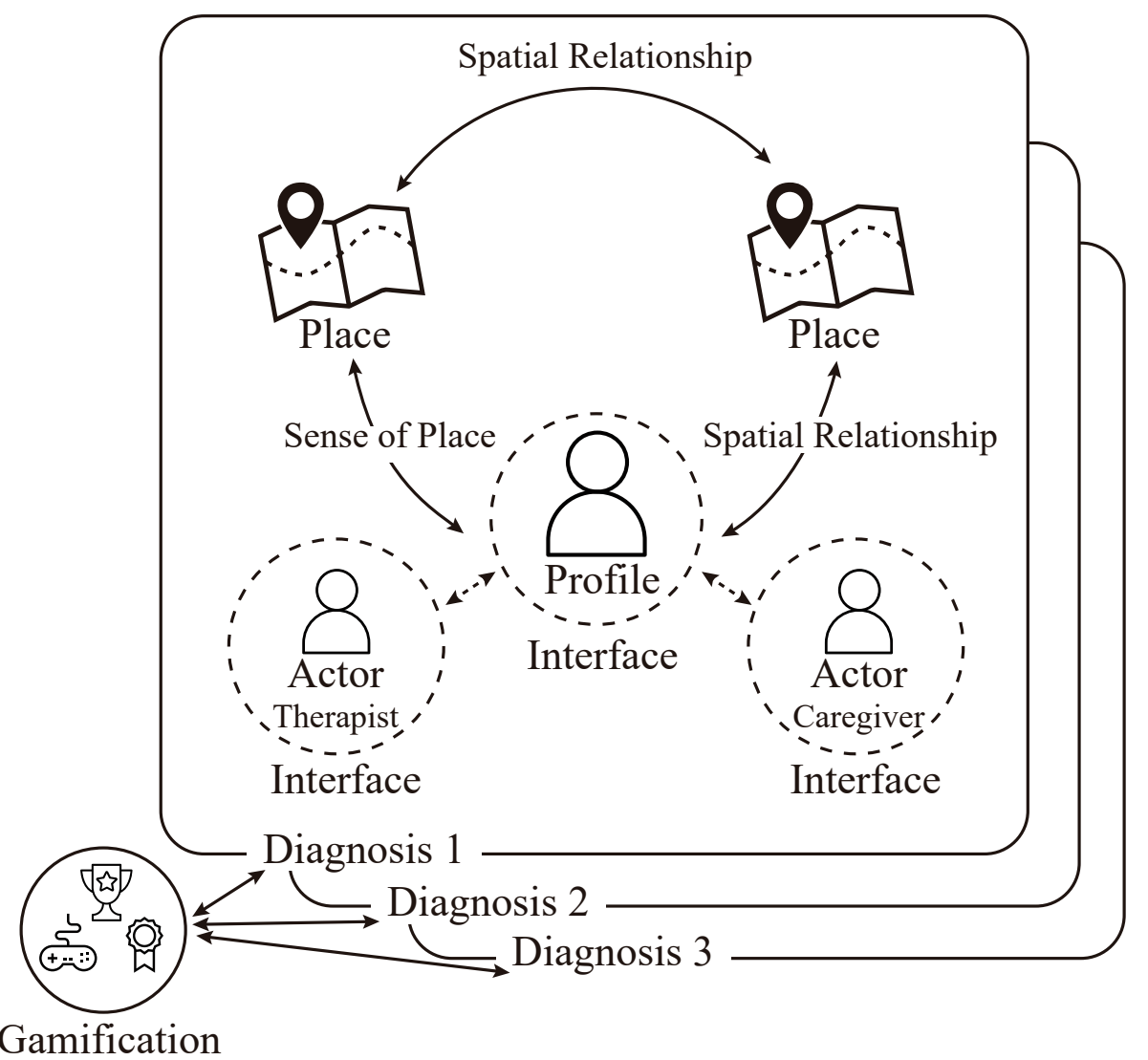

Figure 3.3: Relationships between dimensions. The diagnosis dimension drives the remaining dimensions, since different diagnoses bring different ways of organising the other dimensions to design context-aware mobile applications as intervention tools.

\section{Technology and Context}

The three dimensions within the context area respond essentially to the question of where?. This question is inevitable in the case of context-aware applications deployed in outdoors environments. When observing the link between the dimensions, the relationship between place and spatial relationship emerges naturally.

Places can be visited in a particular order, establishing a temporal relationship between places. In addition to spatial and temporal associations, social 
and emotional bonds to certain places are especially relevant for mental health interventions. For example, the sense of place dimension can categorise places according to the feeling and emotion perceived by patients, producing places of "emotion", places of "anxiety", places of "fear", etc. These types of relations permit us to capture relevant meanings and associations to places, transforming them into personal and unique places.

Through the focus groups, we discovered that virtually all places have the three types of relationships, not necessarily equally balanced, because each of these relationships can be weighted differently for each place. The point was that these three relationships were rarely considered together in the design of psychological interventions. However, one participant suggested that the information relative to these relationships of place "would be most beneficial if it somehow appears on a map because that map would be really useful and visual". Not event the spatial relationship between places (proximity, etc.), which is an objective observation, has been considered as a key design variable in psychological interventions, a fact widely corroborated in the literature (section 3.1).

Various factors may explain the above. First, the assumption that a place has a spatial connotation is widely accepted, but the problem arises on how to spatialise a place, i.e., how to define the precise spatial boundaries of a place attending to the sense of place $[253,270]$.

Second, some places may be selected by therapists for the emotional links they produce with patients, such as places that produce panic and places that should be avoided due to gambling addiction. However, these places are usually seen under the dominant relationship, whether spatial, temporal or sense of place (emotional), neglecting the role of other relationships with that place. Consequently, the three dimensions of the context are largely unexplored, especially when considered together.

The interplay between place and interface is well researched in some specific fields like location-based services [271]. The communication and provision of timely, contextual information depending on the location of the mobile device and/or user, is a defining characteristic of location-based applications, which undoubtedly should be an integral part of mobile psychological interventions. To this respect, some research works have deployed easy-to-implement features, such as letting patients respond to questions, offering textual information and watching media content via mobile devices $[272,273]$. However, much attention 
Chapter 3. Considerations for designing context-aware mobile apps for mental health interventions.

must be paid to the development of more natural and non-intrusive location-based interfaces. This is clear, for example, for the delivery of personalised content and/or to take actions according to the distance (near/far) to a place, how much time a patient spends in a place, how long it takes to go from one place to another, or how often a patient visits a place, just to mention some situations. A patient can approach, enter, cross, stay in, and leave a place and this triggers an action that is made tangible through an interface. Although the distance travelled by a patient is not usually a measure or variable required in the treatments (except those related to physical activity), a participant of the focus group explicitly mentioned that the identification of special places "is very interesting for addiction, in the sense of detecting 'dangerous' places of a patient". The same participant added that "the possibility of providing different levels of activation and/or alarms [when a patient is in these special places] is a really relevant and useful feature for the interventions". These are the type of interactions that can be developed when the dimensions of places, the spatial relationship, the sense of place and the interface are considered together. Therefore, the potential combinations between these dimensions are countless, and they must be carefully thought about for the intervention at hand, considering also the profile dimension, since any triggered action is directed, i.e. is customised, to a profile (i.e. a patient) through an interface.

The interrelation between place and gamification is also mediated by the spatial relationship and the sense of place. Sharma et al. [274] wondered how game designers select physical spaces to create places. In their review, the authors developed a detailed classification for the use of space in mixed reality games, which can also be understood according to the spatial (and temporal) relationship and the sense of place. In summary, the re-examination of the dimensions of technology and context allows therapists to walk through unexplored routes to discover new options for the design of mobile tools for mental health interventions.

\section{Context and Mental Health}

In the realm of psychological treatments, capturing, understanding and managing context, in order to better assess and intervene, is the ultimate goal. In general, the importance of the context was well recognised by the participants of both focus groups. The discussion turned to more general considerations relative to context such as, for example, what makes a place safe or, at least, a place that 
patients consider or perceive as safe. Safety can be seen from different points of view such as traffic, pollution, deterioration of buildings, and dirty streets, being a major problem in modern cities and, therefore, in the development of interventions in urban areas. Again, a good understanding of the structure and organisation of the city or urban area where the intervention is planned to be developed is vital [275]. The selected places for the intervention must be carefully considered. As one participant aptly summarised "if patients feel the app is going to help, they will accept it". An app forcing patients to go to unsafe places may produce undesired effects on patients.

Participants also casted doubts about which features of the context were more relevant for each intervention. A variety of ways to collect contextual information exists, i.e., using sensors and analysis of the obtained data, using location-based services, communicating with peer devices in the surrounding environment, or asking the user [276]. Multiple examples from the literature demonstrate the degree of penetration and use of sensors in mobile devices for data collection, analysis and context determination. That, of course, also applies to psychological treatments that are provided to people in real-time during their everyday lives and in natural settings, anywhere [226]. In depression, for example, contextbased techniques to recommend activities have been proposed [277, 278]. Other examples capture the location information of patients to detect whether they are in a remote place, so that reminders or suggestions are sent accordingly [124]. The focus of Simm et al. [279] is on anxiety, and the authors use contextual information to allow patients to relate visually, through a mobile app, places to symptoms, linking them to the sense of place dimension. Finally, Skillen et al. [69] address caregivers (actors) related to patients with autism so that they can establish areas (linking again to the place and spatial relationship dimensions) where notifications are sent when the patient enters or leaves them. Once participants were explained the diverse methods to gather contextual information, they immediately suggested potential scenarios such as "the collection of location data of the patient in indoor places for obsessive-compulsive behaviours". Another participant suggested that "the detection of manic phases in bipolar patients" could be a plausible scenario "since the monitoring of high activity can be an indicator". 
Chapter 3. Considerations for designing context-aware mobile apps for mental health interventions.

\section{Mental Health and Technology}

Gamification research, seen as a collection of game features, game elements, and mechanics that aim to motivate and engage users [266], involves a wide arsenal of theoretical approaches and practical actions and techniques [267]. Here we are not interested in any game scenario in general, but in mental health interventions in particular. Therefore, the following question focuses on the connections between gamification and profiles: What gamified characteristics can make interventions more attractive to patients (players)? An approach to answer it may be to analyse and segment target users to select the game elements and gamification techniques that better suit the needs of each user segment. In his seminal study, Bartle [268] associated the main characteristics of video games to user profiles, to create general categories of players such as the "explorer", "socialiser" or "achiever". For example a socialiser player is specially interested in interacting with other players, and an achiever is focused on acting on the world. More interestingly, Bartle [268] established the path to enable connections between user profiles and game elements. Along this line, Tondello et al. [269] recently carried out an empirical exploratory research of the preferences of players in games, which resulted in nine game elements and five game styles associated with certain user profiles.

The below examples analyse games and player profiles together. These results do not imply that all game strategies and elements should be completely used for a user profile; it is simply advised to take them into account if a positive impact is expected on the effectiveness of the gaming applications. Yet, the aim of these examples can be extrapolated to mental health scenarios where adherence to use of a mobile device as part of a treatment is a priority. Mandryk \& Birk [280] study the potential impact of game-based interventions on mental health, paying attention to the relationship between game elements and self-reported indicators of wellbeing by players (patients). The results suggest that games are a motivating attraction and provide considerable appeal, regardless of the age and gender of the participants, which shows that gamification is not limited only to young people, but prevails in all demographic groups.

Figure 3.4 summarises the results of the RQ2.1 (identification of relevant dimensions) and RQ2.2 (meaningful relationships among dimensions). It gives an overview of the relationships between the dimensions and aspects that affect the 
design of context-aware mobile apps for mental health interventions, which will be discussed further in the following section.

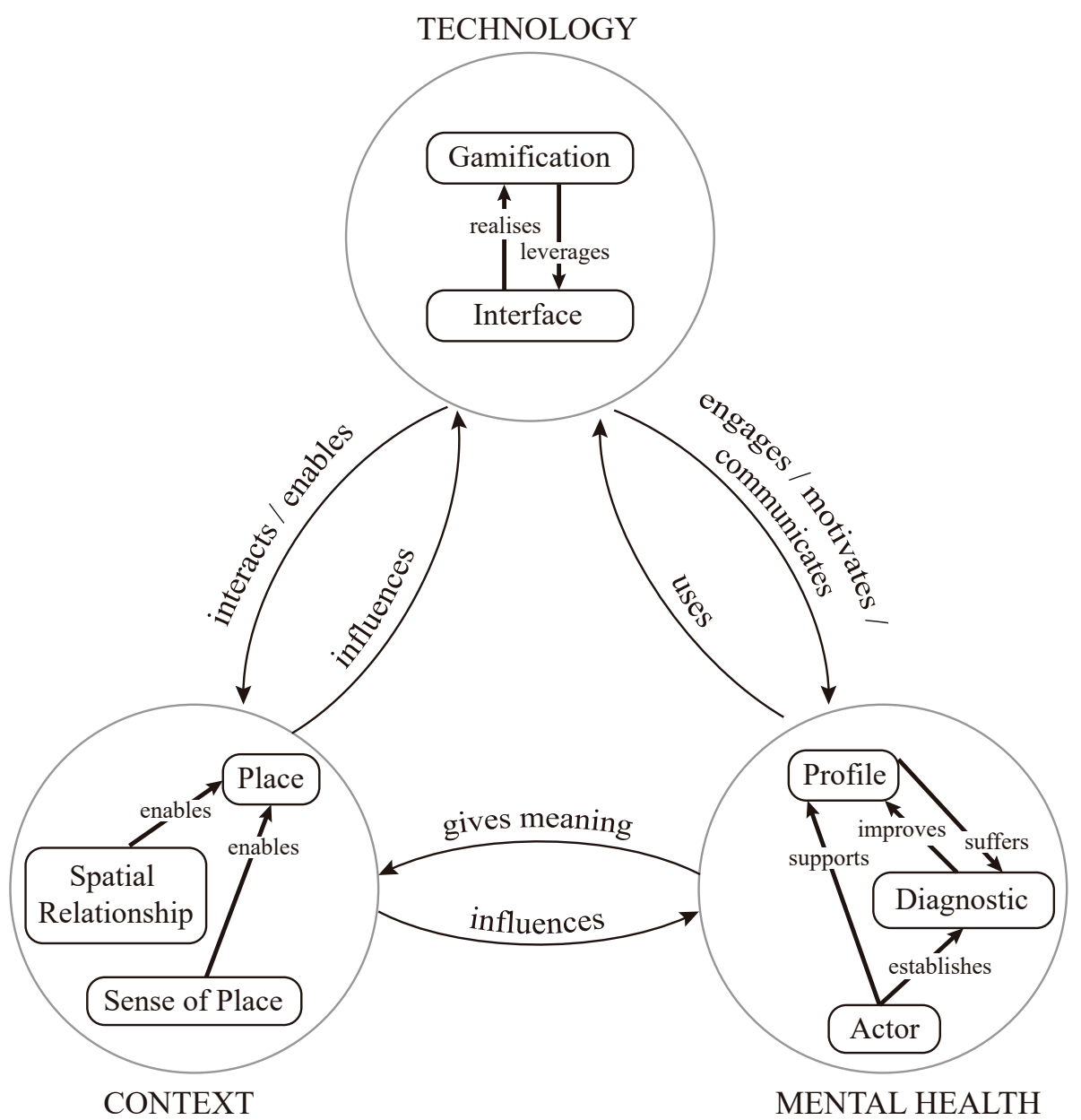

Figure 3.4: The design process of context-aware mobile apps is influenced by a number of interrelated dimensions, that need to be considered as a whole.

\subsection{Discussion}

In this section we address RQ2.3 - which interrelated dimensions may best inform users for designing context-aware mobile apps to support mental health intervention tools? We discuss the interrelation between the aforementioned dimensions and provide some considerations screened against the literature, when possible, as well as limitations of the present exploration. 
Chapter 3. Considerations for designing context-aware mobile apps for mental health interventions.

\subsubsection{Considerations [RQ2.3]}

Despite the unquestionable potential benefits of mobile apps for mental health assessment and treatments, a recent study concluded that "[o]verall there is a significant disconnect between app developers, the scientific community and health care, leaving the utility of existing apps questionable" [222, pp. 526]. The authors, who conducted a comprehensive literature review of the current state of mental health apps for anxiety, depression and mood disorders, refer mainly to the multidisciplinary nature of the $\mathrm{m}$-health field, in which research teams with different backgrounds and skills are more necessary than ever. Therefore a fluid communication of scientific, technical, social and psychological information between team members and patients is a must. A good deal of research has shown the feasibility and benefits of the application of participatory and human-centred design in m-health [279, 281].

We agree with the previous statements and argue that the design, implementation and delivery of context-aware mobile apps for mental health intervention are by nature a multidisciplinary and multidimensional field. Nevertheless, it seems clear that other aspects and factors can make a mobile application fail as an intervention tool. Our message here is to make developers and psychologists aware of the relationships between these interdisciplinary dimensions in order to mitigate unnecessary failures during the design process and the subsequent implementation process of mobile interventions. While we have noted that substantial research has been conducted in many of these dimensions separately, we have also identified that a more holistic approach for designing mobile interventions is a clear gap in the literature, which we attempt to fill here. In the following, we discuss how the relationships represented in Figure 3.4 can lead to a set of considerations to help developers and therapists design mobile interventions for mental health wellbeing.

Designers, psychologists, geographers and urbanists should work together to carefully consider the multiple relationships to places when selecting and incorporating places in mental health interventions [274]. The creation and identification of places, which gather subjective perceptions of the interaction between people and the environment and place value on them, can have a great impact on the effectiveness of mental health interventions. The availability of methods for the spatialisation of place and place-related concepts like sense of place is gaining 
importance, and practical approaches to compute it are under way [282]. This will undoubtedly facilitate the task of establishing spatial boundaries of places to take advantage of the dimensions of spatial relationship and sense of place that will be enabled through interfaces.

As places are central, a logical consequence is that locality is crucial for the success of context-aware mobile apps. It is expected that an intervention will be personalised to the places where it is deployed. In other words, a context-aware mobile app that leverages on local places cannot be experienced anywhere, since the affordances and emotional bonds created and generated with these places are specifically designed for the local urban configuration and the profiles of the patients. Much attention should be paid to the cultural, social, urban morphology, and demography differences between cities. In addition, as patients are also stratified according to socioeconomic and cultural variables, The identification of places is not only based on the structure and organisation of the urban areas, but designers must also take into account the profile dimension to choose the right places.

The profile dimension uses and interacts with the interface, regarding the latter as an envelope of the sensing, communication and interaction capabilities of mobile applications or devices [283]. The interface performs or implements the gamification dimension. The combination of the dimensions of interface and gamification is critical to augment the potential of interfaces to deliver content in novel ways to profiles (patients) and/or motivate them for sustaining adherence and engagement over long periods of time. These two features are much sought after by actors (therapists). In other words, gamification strategies applied to mobile applications for mental health interventions can be considered as "activators of social and health change", as experimentally observed by Spallazzo \& Mariani [233]. The authors also pointed out that gamification can be considered as a practice capable of impacting both designers (or therapists) and players (or patients) to establish multifaceted relationships between them. Therefore, taking into account the dimensions proposed and carefully considering the best way to relate them, it seems to be a recipe for successfully designing specific interventions. The proposed holistic process provides a wide range of means and strategies to carry out the chosen psychotherapy approach to influence patients and change their behaviours in the directions determined by the therapists. 
Chapter 3. Considerations for designing context-aware mobile apps for mental health interventions.

\subsubsection{Limitations}

We have discussed here that the design of context-aware mobile apps for mental health interventions is affected and influenced by a reduced but comprehensive number of influential dimensions at a high level of abstraction such as place, spatial relationship, sense of place, interface, gamification, diagnosis, profile and actors. As usual, the devil is in the detail. We have set aside much more detailed aspects and factors that must also be considered at later stages during the development process. For example, while the dimension gamification is appropriate at the time of design, specific game mechanics, strategies and elements must be specified at some point in the development of a mobile intervention. The same is true for the dimensions of places and sense of places, which must be clearly specified during the development of the intervention. The key point is that all of these dimensions must be carefully considered if context-aware mobile applications are used as intervention tools. Therefore, the dimensions, their relationships and design considerations do not allow designers, physiologists, and practitioners to implement a mobile intervention, but rather they intend to help them consider an integral interdisciplinary strategy when designing context-aware mobile apps for mental health interventions.

We also realise that a limitation of the chapter is that the research approach adopted is eminently qualitative and, therefore, there is a lack of experimental data to validate our findings. This will be addressed in future work as we are planning a series of experiments to test distinct mobile interventions for mental health diagnoses taking into account the dimensions and design considerations presented in this study. On the positive side, however, we examined diverse sources through the document analysis method, along with other qualitative research methods (Section 3.2). This approach proved to be effective in practice to verify information from multiple documents and research papers, and helped us to order and reinterpret statements in the analysed documents.

\subsection{Conclusions}

Through a methodological approach that combines several qualitative research methods, we have re-examined key documents to identify a set of relevant dimensions of the three areas of knowledge (Technology, Context, and Mental Health). 
Next, we have reinterpreted the potential relationships between these dimensions, both within the same area of knowledge and between different areas. Finally, we have provided a set of considerations and validated them against existing literature. When therapists and developers strive to design and create mobile apps for mental health interventions, it is advisable that they take into consideration our findings for designing custom mobile applications for mental health interventions. Adhering to the holistic strategy described here can increase the possibilities of designing mobile interventions that keep patients motivated and engaged and, therefore, increasing the effectiveness of the psychological treatment.

Wykes et al. [192] argued that funding research on e-health and technology applied to mental health interventions "generates a good return on investment, reducing not only the burdens on individuals and families but also the costs of care and support in the long term" [192, pp 2]. Our intention here is to attract the attention of developers and therapists to comprehensively consider the multiple interactions between the relevant dimensions of all areas of knowledge, as a means to capture physical and social context. Instead of concentrating and trusting only a few, they need to expand and make the diversity of dimensions and their relationships a strategy to discover new options for the design of context-aware mobile tools for mental health interventions. 


\section{Development of a builder tool for customizing location-based, gamified apps for depression.}

A new power is rising. Its victory is at hand.

Saruman the white.

Depression is worldwide the most prevalent mental disorder, affecting hundreds of million people and being the leading cause of disability. In lack of sufficient psychological personnel and to improve efficiency, technology-assisted treatments are increasingly being used to help patients. In particular, smartphone-based applications have proven to be a useful complement to traditional treatment, especially when applying adherence techniques based on gamification. However, due to the technological complexity of app development and its need for a multidisciplinary (psychology - informatics) approach, the availability and versatility of such apps is lagging behind. A compound software development methodology was applied. First, a focus group, consisting of psychologists, was used to perform an initial requirements elicitation. Subsequently, the agile development methodology SCRUM was used to progressively develop the platform, receiving feedback through faceto-face meetings with psychologists after every development cycle. This chapter presents a software platform that allows therapists to generate and configure their own location-based, gamified applications to perform interventions for patients with depression. These apps collect patient's data in real time, trigger location-based personalized messages and apply a gamification strategy as configured by the therapist according to the patients' needs. The platform gives therapists a tool to 
4.1. Introduction

develop location-based gamified apps for the treatment of depression, without the need for a lengthy, multidisciplinary software development process. Therapists testing the platform and generated apps were very satisfied and show a high intention to use the tool in their therapy.

\subsection{Introduction}

Depression is one of the great problems that humanity is currently facing [3] and will be facing in the coming years [189]. According to the World Health Organisation (WHO), "over 300 million people are estimated to suffer fro, depression", and depression is the leading cause of disability worldwide, and a major contributor to the global overall burden of disease." [284]. Next to the adversely impact on people's well-being, it also has a significant economic impact. For example, Evans-Lacko et al. [4], indicated that the cost of mental disorders in the EU-25 was $€ 72$ billion in 2010 .

Psychological treatments have been researched and established over the years to battle mental disorders [285] in general, and depression in particular. One of the dominant forms of psychotherapy is Cognitive Behavioral Therapy (CBT) [258], whose interventions have been experimentally proven to significantly improve the mental health of people. Among the components of CBT, literature shows that behavioural activation (BA) is essential in the treatment of depression, because its efficacy is equivalent to complete treatment [286, 287]. BA interventions are therefore well-established, effective treatments [288] for major depressions [289].

Recent technical advances and developments in mobile devices and built-in sensing capabilities opened the door for a new generation of psychological interventions in which the momentary and ecological features of the mobile technology are exploited to monitor, evaluate and intervene in mental disorders. Recent reviews to this matter highlight this trend; Thomée [290] evaluated 290 academic studies in which technology played a key role in interventions for mental disorders (including depression), and I et al. [291] conducts a systematic review on the availability of smartphone apps for the treatment of mental disorders, resulting in 111 studies from 2013 to 2018. Clearly, mobile applications and related technology (e.g. advance analytics, data collection methods, real-time interactivity and communication) have grown exponentially and, consequently, it seems plausible for the research community to take advantage of these technological advances to explore 
Chapter 4. Development of a builder tool for customizing location-based, gamified apps for depression.

novel approaches to enhance the efficacy of treatments and patients' satisfaction. Even though there are some incipient examples that have fully embraced the characteristics of the new medium to enhance the treatmetns (e.g. [292-294], as a general rule of thumb, these new characteristics are presently underutilized [291].

One point of attention to further reinforce the uptake and usefulness of mobile device-based interventions is how to increase the patient adherence to the mobile app [229]. To this extent, serious games and gamification are promising techniques with the potential to engage people and extend the reach of treatments to an audience otherwise not reached.

In the context of mental health, [295] reviewed the interplay between serious games and depression, concluding that the development of such a kind of tools was feasible and, most importantly, that people were willing to try them, especially young people. The authors argued that the latest data suggest an increase in global access to game applications and in the adherence they offered. Game techniques have also been used to predict which game features and elements are more suitable for certain profiles [230], tailoring them to the peculiarities of patients.

The application of game techniques and elements to the treatments of depression has been explored previously. Stallard et al. [296] developed a game tool (Think, Feel, Do), designed to be facilitated by a professional, through which children aged 11 to 16 years linked thoughts and feelings to differentiate positive thoughts from negative ones in an accessible and friendly way. SPARX (smart, positive, active, realistic, $x$-factor thoughts) [297] was a Computerized Cognitive Behavioural Therapy (cCBT) program that focused on adolescents with depressive symptoms. In it, a user avatar in a virtual world had to face and overcome challenges in order to get rid of the world of negativity. Another example is the Journey, a Flash-based game developed by Stasiak et al. [298] in which the user travelled through a virtual world and obtained points by completing modules of therapeutic content related to depression. Reach Out Central [299] was an online serious game for young men (16-25) to encourage them to identify strategies to improve their mood. The game included non-playing characters (NPCs) with whom to interact; player's responses affected both his mood and that of the NPCs. This brief selection of examples shows that the incorporation of gamification techniques and strategies into interventions can lead to increasing levels of adherence to treatments. 
Besides the issue of adherence, a second problem in the current wave of mobile device-based interventions is a lack of use of location analysis and locationbased technology [300]. Location-based games, also known as location-aware games [301] or geogames [302], are games whose mechanics involve georeferenced elements and resources, i.e. elements that are contextual to the surrounding environment. One of the most famous example is PokemonGO, in which a user navigates in an augmented reality world to capture virtual creatures and make them fight. PokemonGO did not discover the benefits of location in games. Early adopters were Geocaching [303], Zombie Run [304], and Ingress [305], while recent examples such as Origami [306] and Turf Wars [307] embrace a variety of location-based technology. In one way or another, all of these games take into account location as an integral part of the game mechanics. The advantages are several; social relations, concentration, and outdoor physical activity have been improved when users are engaged with location-based games [308]. In addition, recent studies have shown that location-aware games enhance collaboration [309] or even relationships between family members [310] through collaborative learning techniques [311]. Experiences with location-based games show that the combination of gamification with geo-referenced elements and location-based technology not only provide new possibilities for entertainment, but they can lead to great advances related to health, socialization and collaboration [302, 312].

Built on previous work where a set of considerations for designing contextaware mobile application for mental health interventions were discussed [300], the objective of this work is the design and development of a platform to allow health professionals to create, in a simple and intuitive way, mobile applications for the treatment of depression. The generated mobile apps are able to include gamification techniques to improve adherence and also take advantage of patient's position and location analysis to promote behavioral activation. However, having a reliable activity-monitor system makes the technological development more complicated due to the addition of gamification and location-based technology on top of it. Although there exist some examples [313,314], it seems prudent to accept that the field remains largely unexplored.

Followed to this introduction, the methodological approach is described in Section 4.2. Next, the complete technological platform is detailed in Section 4.3, as well as the tools used for its development. Finally, the strengths and limitations of 
Chapter 4. Development of a builder tool for customizing location-based, gamified apps for depression.

the research are discussed in Section 4.4, along with final conclusions.

\section{2 $\mid$ Methods}

As the main outcomes are a software platform and tools, the methodological approach is based on well-established software engineering methods. Because we did not pursue the development of a particular app or game but a generator of gamified, location-based mobile applications, the scope, intent and granularity of the requirements and needs differed substantially from those for the development of concrete, ad-hoc applications. Therefore, we combined software engineering methodologies (Section 4.2.1) with qualitative research methods for requirement and knowledge elicitation (Section 4.2.2).

\subsubsection{Software engineering methods}

The design and development of the platform and associated tools was carried out in cycled iterations following the SCRUM agile methodology [315]. Contrary to traditional software development methodologies, an agile methodology allows to adjust project requirements and expected results during the course of the project. This way, the development team can react quickly to unexpected situations or changes of the project requirements, in order to avoid excessive delays in the delivery of a functional software tool or service. In SCRUM, the basic temporal unit is a sprint which may be thought of as a mini-project that goes through all relevant phases in software development during a short period of time. As such, the final tool or service is progressively developed step-by-step in small increments in a series of sprints. In each sprint, a limited set of new characteristics are defined, agreed, developed, tested and presented to interested parties, with the objective of obtaining comments and points of improvement and refinement for subsequent sprints.

For the development of the platform and tools, we took on most of the core concepts of the SCRUM methodology such as the development in small increments (i.e. sprints), user stories, frequent feedback and user-centred design, among others. Other SCRUM concepts (e.g. daily meetings, sprint retrospective meetings) were discarded because they were considered counterproductive to reach the objectives in a reasonable time. That decision does not contradict the SCRUM 
methodology, but it is fully acceptable since SCRUM is a flexible methodology that can be adapted to the often-changing circumstances of each project.

As mental health practitioners and psychologists are the primary users of the platform, it was essential to understand their work and their specific needs before entering into the development phase. Therefore, we established a few but robust requirements (see above) that drove the planning of the sprints, and were subject to fine-grained refinement during subsequent sprints.

\subsubsection{Qualitative methods for requirement elicitation}

We started the phase of requirement elicitation by conducting interviews with a group of mental health practitioners and psychologists (authors: JBL, DC) of the research group LABPSITEC (Psychology and Technology Laboratory, [316]. This research group is specialized in applying technologies to psychological treatments, so their members have an appropriate profile to make informed decisions about the needs of the sought platform. These interviews allowed us to extract basic needs and requirements as well as to establish a shared framework to enable a fluid communication of scientific, technical, and psychological information between the two disciplines. The latter is not a banal aspect but primordial to ensure the success of interdisciplinary research. A recent literature review about mental health apps for anxiety, depression and mood disorders, concluded that "there is a significant disconnect between app developers, the scientific community and health care, leaving the utility of existing apps questionable" [222, pp. 526].

Once the communication framework was set up, a focus group followed to set out the needs and requirements of the platform and tools (see details in [300]). In the focus group eight psychologists from the LABPSITEC group were involved. Following the focus group methodology [214], participants were asked to discuss the requirements for the assessment, monitoring and intervention with smartphone applications. Participants were encouraged to consider the geographic elements and location as drivers of the discussion. The focus group was recorded and adhered to standardized protocols to ensure ethical considerations of the participants. 
Chapter 4. Development of a builder tool for customizing location-based, gamified apps for depression.

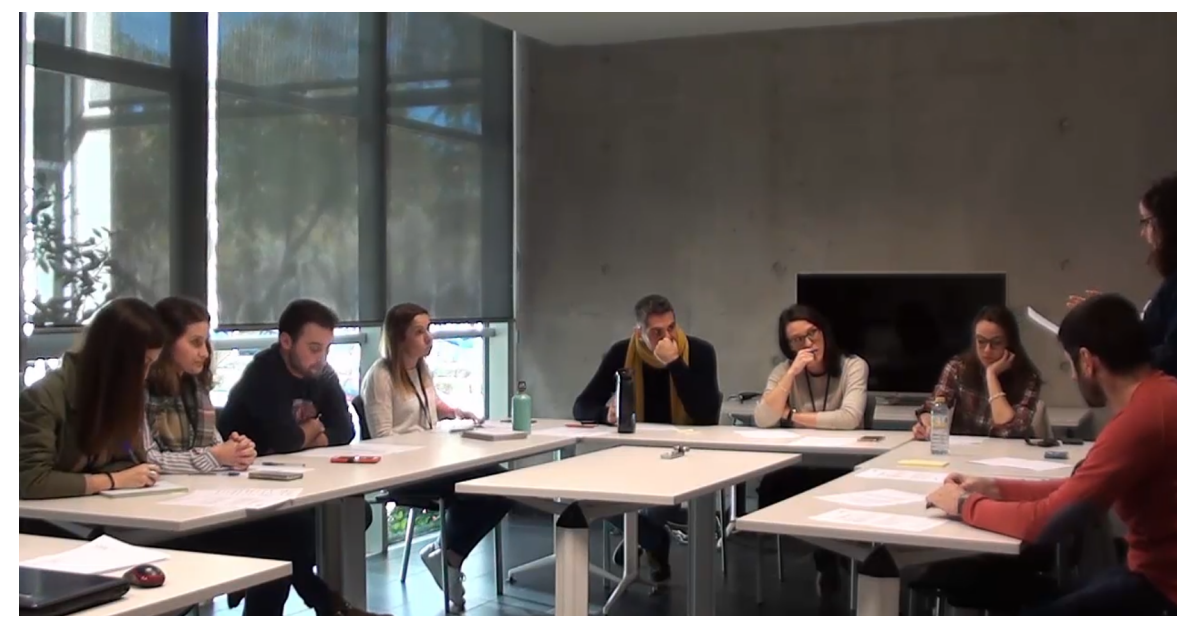

Figure 4.1: A moment of the Focus Group meeting in which the participants discussed specific requirements of the platform.

The combination and integration of the results of the interviews and the focus group yielded a list of main requirements $(R)$, which have been taken into account for the organization of sprints and hence for the development of the platform and tools:

R1: The mobile app should be able to identify and monitor how many times a patient leaves the house.

R2: The builder should allow the therapist to customize the number of times recommended for each patient, messages received and the time at which the messages should be displayed.

R3: The mobile app should allow the patient to check how many times she has left home and compare it with the therapist's recommendations.

R4: The mobile app should allow the patient to receive daily feedback.

R5: The viewer should allow the therapist to query and visualize the patient's monitored data and contextual data gathered by the app.

Once defined, these functional requirements were interpreted in the realm of the design considerations stated in Miralles \& Granell [300]. In that work, three major areas of knowledge along with their relevant dimensions were discussed (Table 4.1). These considerations are not intended to be an implementation guide 
but rather a series of evidence-based recommendations to increase the chances of success for the design of location-based, context-aware mobile applications for the treatment of mental disorders. Therefore, the elicited requirements framed with these recommendations articulated the entire development phase (Section 4.2.1), in the sense that these requirements and recommendations were contrasted at the end of each sprint to ensure the intermediate results were as close as possible to the envisioned ones. A certain degree of flexibility was encouraged, though, to enable changes and adaptations when required but in close conversion and agreement with the therapists. All in all, the platform and tools we have achieved in this research closely resemble the ones that the therapists thought of during the first interviews.

Table 4.1: Considerations for designing context-aware mobile apps for mental health interventions [300].

\begin{tabular}{ll} 
Area of Knowledge & Relevant Dimensions \\
\hline Technology & Interface and Gamification. \\
\hline Context & Place, Spatial Relationship and Sense of Place. \\
\hline Mental Health & Profile, Diagnostic and Actor.
\end{tabular}

\section{3 $\mid$ Results}

The platform is conceived as an underlying infrastructure in which an ecosystem of interrelated tools and services sits. Three tools stand out: a builder tool for the therapist to configure and generate an operational mobile application; the mobile app generated, which is used by the patient to collect contextual and patient's data; and the viewer tool, which allows the therapist to view and monitor collected data from the patient. This section pays special attention to the builder tool and the mobile app, and how they address the requirements elicited in Section 4.2.2. We partially cover the viewer tool and the underlying infrastructure of the platform, to highlight the connections with the builder and mobile app.

\subsubsection{The builder tool}

Mental health practitioners, who often do not possess developing and technical skills, can use the builder to create their own location-aware gamified mobile 
Chapter 4. Development of a builder tool for customizing location-based, gamified apps for depression.

applications. The goal is to accelerate their research and drastically reduce the time required to deliver mobile applications as part of treatments. The builder assists therapists to configure and personalize the resulting mobile application to the needs of a patient or group of patients, addressing R2. Therefore, no programming skills are required and the output of the builder is a location-based, gamified mobile app for the patients.

From the therapists' perspective, the builder allows them to focus on the design and delegate entirely the implementation details to the builder. Design here means that the therapists take into consideration all the relevant dimensions [300], and determine the best way to relate them to the particular needs of the patient(s). For example, it is strongly advisable for therapists to answer the following questions in the realm of the expected functionality of the mobile application: How do the Profile and Diagnosis dimensions relate to the Place and the Spatial relationship ones? To put it short, are certain places important to the diagnosed disorders? Can these places be spatially identified (e.g. a park) or are ambiguous to the patient (e.g. zones without clear boundaries)? Do these places gain importance as a patient is close, distant, or just right there?. Another key question is: How does the Place dimension relate to the Interface dimension? That is, whether (and how) the application interacts and communicate with the patient depending on where the patient is. Does a patient get notified when he is in a predefined place, or when he is approaching (leaving, crossing, etc) it, or when that place is never visited?

While finding the connections among these dimensions (i.e. Technology, Context and Mental Health in Table 4.1) may be hard work at first glance, they are in fact pre-established by the application template provided by the builder. In other words, each clinical choice in terms of a diagnosed disorder (depression, anxiety, panic, etc.) entails an application template that in turn predefines a set of relevant dimensions and the relationships between them. As a result, an application template guides therapists in designing an application ensuring that the vital dimensions and relationships among them are considered in the final mobile application. Therapists must concentrate only on the customization process of the builder tool to personalize the application template into a functional mobile application. This way, the builder can generate multiple slightly variations of the same application template based on each therapist's settings. The builder currently supports one application template, which meets R2 and targets population with 
depression. Other application templates will be supporting in the future to address other types of mental disorders.

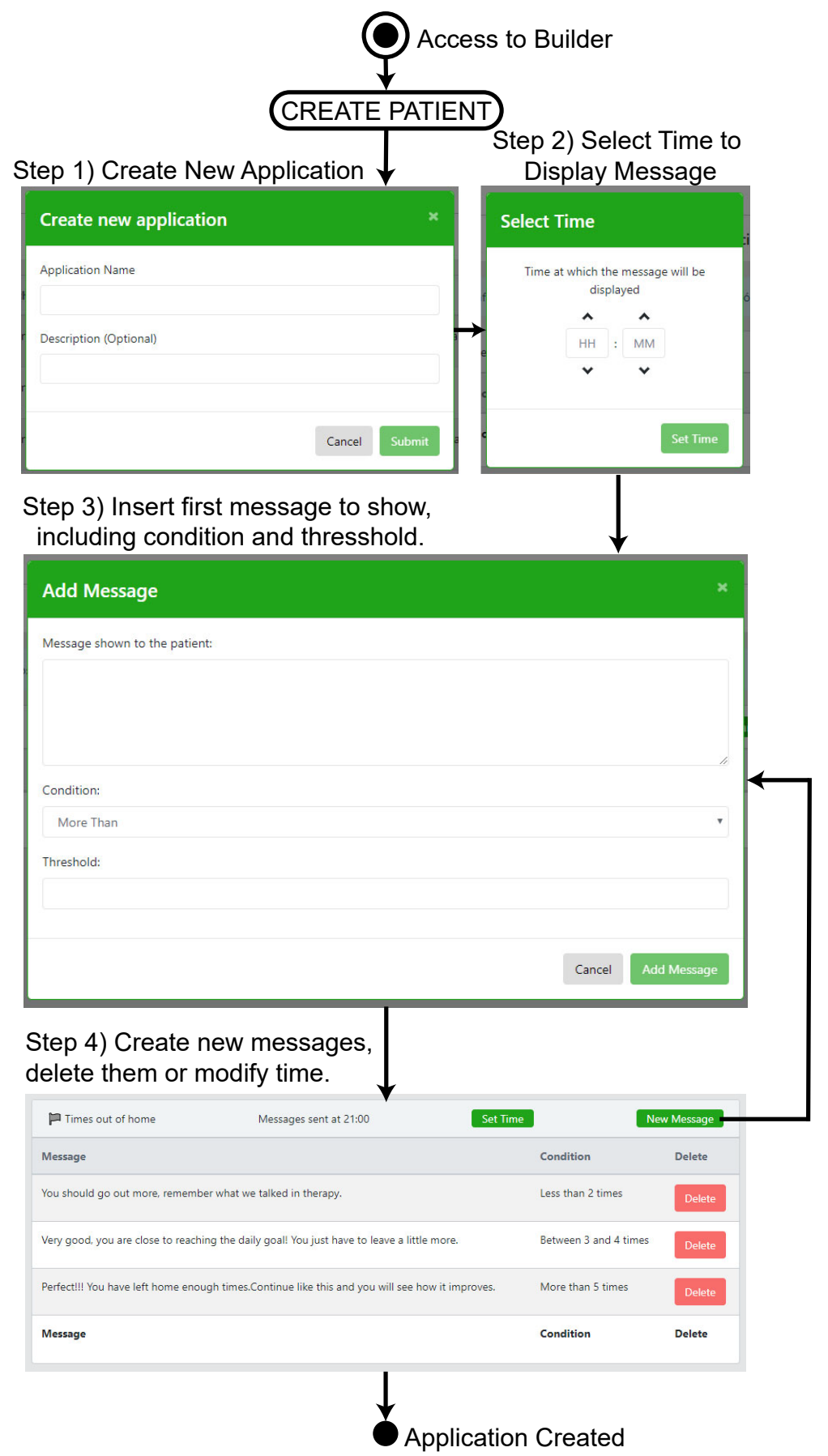

Figure 4.2: Flowchart diagram of the process to create a new application using the Builder tool.

Figure 4.2 describes the entire process of the application creation. Using 
Chapter 4. Development of a builder tool for customizing location-based, gamified apps for depression.

a browser application, the therapist accesses the web-based builder tool that requires valid credentials. Once authenticated, a therapist registers the patient(s). We strictly followed standardized mechanisms to ensure that concerns about privacy and personal information are handled appropriately. For example, the tool does not store any personal identification data of the patient, but a unique and anonymous identifier is generated per patient to relate records (patients to therapist, patients to apps) in own databases. Data storage and persistence comply with ethical and privacy data regulations.

The configuration process starts by requesting the name and description of the app (Figure 4.2, step 1). The next step is to enter the time of day at which the therapist wants the patient to be notified with the updated information of their daily progress (Figure 4.2, step 2). Although this sort of notifications are usually sent at the end of the day, it is up to the therapist to decide the best time to notify the patient. Alternatively, the app allows the patient to access these notifications at any time because the patient may not remember (or even not know) the number of times recommended by the therapist. When a patient checks the progress bar, s/he can recall whether it is needed to go out for that day to get the success message.

Timely and spatially accurate notifications are strictly necessary. The therapist can add new messages (i.e notifications) by filling the fields in Figure 4.2, step 3. The first configurable field is the content of the message delivered to the patient which is a free text field. The Condition specifies a certain condition that must be met. For example, the type of conditions expected is like leave home more than (or less than or between) a certain number of times in a day. The second part of the previous sentence is defined by the Threshold field, that permits a therapist to specify a number of times in a day. Therefore, the three fields together define simple rules of the form if-then, where the combination of the fields condition and threshold specifies the if-clause and the message the then-clause. Finally, a therapist can also manage the list of personalised messages (Figure 4.2, step 4).

It is important to note that the generated app (an APK file) is not really being compiled, although that option was originally considered. Compiling an app each time would have increased the technical complexity of the platform, as well as the publication of the mobile app in the Google Play Store. In contrast, a highly and acceptable level of personalization can be achieved by configuring the input parameters of one application. These parameters are for example the definition 
of custom messages, the detection of the patient's house, and the exact time to launch notifications. Each application template defines one app; the builder personalizes it according to the therapist's selection to create the final app. In consequence, the builder carries out quite a lot of steps behind the scene, which are fully transparent to the therapist. That's why we decided to use the term "Create new Application" instead of "Create new Configuration", for the sake of clarity.

Once the configuration process is completed, the configuration file is stored and the application is created. The final step is to relate that application to the patient (or patients, in case the therapist wishes to apply the same configuration to many). When carrying out this Patient-Application relationship, a unique code is generated for each relationship. This code is then introduced in the first execution of the application to be properly configured for the treatment.

\subsubsection{The mobile app}

The builder generates a mobile app which is configurable according to the therapist settings to satisfactorily meet the requirements $R 1, R 3$ and $R 4$. It is available for download from the Google Play Store, so a patient can easily install it in any Android-based device. Two key steps are necessary to turn the generic app into a customized one ready for intervention: activation and configuration. When the app is opened for the first time, the users are asked for the necessary permissions to operate such as energy management and access to GPS. In addition, the app requests the activation code, the App Key (Figure 4.3, a) the therapist have generated with the builder tool. Once entered and confirmed, the app downloads the associate configuration file from the platform and installs it to adopt the desired behaviour. From that moment, the customized app is up and running to collect and monitor the patient's data (e.g. movements).

During the first two days after activation, the app only controls the movement of the patient. This is a baseline period to determine the patient's home. The app automatically clusters the gathered movement data and selects the most prominent group (i.e., the most visited area) as the patient's home. Once that place is set, the patient will be receiving a notification periodically at the time indicated in the Builder (Figure 4.3, b). At the time configured by the therapist, the application sends the message associated with the number of times $\mathrm{s} / \mathrm{he}$ has 
Chapter 4. Development of a builder tool for customizing location-based, gamified apps for depression.

left home. The patient sees that number through an interface composed of the message and a progress bar (Figure 4.3, c), as a simple example of the use of gamification elements to motivate adherence to treatment. If at any time of the day the patient wants to know the current status, he can check the progress bar without awaiting for the next notification.

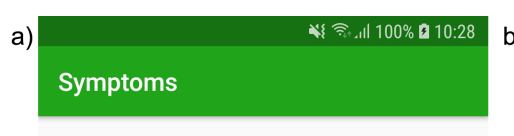

Insert an application key

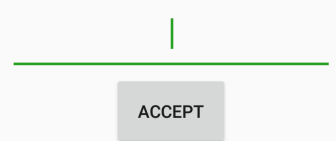

b)

11:30 | Tue 26 February

[3 Symptoms 11:30

Times out of home

You left home 3 times today.

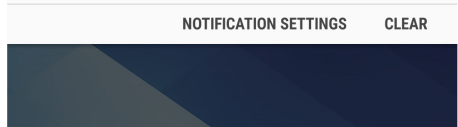

c)

Symptoms

Very good, you are close

to reaching the daily goal!

You just have to leave a little more.

Times out of home: 3

BACK

Figure 4.3: Screenshots of the built application. In a) the screen where the AppKey is entered is displayed; b) is an example of the received notification; $c$ ) presents the interface that is accessed both from the notification and from the app.

The patient can cancel the monitoring at any time, due to unexpected events during the treatment. In addition, the patient can perform a data cleansing in the mobile app, which means that all of the local data stored in the mobile app (e.g. configuration data) is fully removed. Under the supervision of the therapist, this action allows the patient to load another configuration file into the mobile app, for example. Even in the case of cancellation or change of treatment, all the patient's collected and generated data during a treatment is stored and secured on the server-side of the platform, so that the therapist can monitor a patient and analyze the resulting data through a dedicate visualisation tool.

\subsubsection{The viewer tool}

While the builder and mobile app are core tools and tightly connected to carry out a mobile intervention, the viewer can be seen as a complementary tool created to increase the pool of resources for therapists. The web-based viewer allows the therapist to monitor in real time the gathered data of a patient or group of patients through convenient visual means to analyze and explore it, therefore addressing R5. Given the list of patients, the therapist selects one of them to quickly visualize the information that is generated continuously. By showing objective data arranged 
chronologically, the therapist obtains a global trend of the patient's evolution. For example, examining the value of the column "exits" (Figure 4.4), the therapist can assess whether there is a tendency to increase the behavioral activation component.

Figure 4.4 shows examples of feedback sent to the patient during the treatment. The simple interface of the viewer tool makes it easier for the therapist to have better control over information of clinical value. Indeed, through the viewer, a therapist can review the contents of the treatment and launch new messages which best suit to each patients.

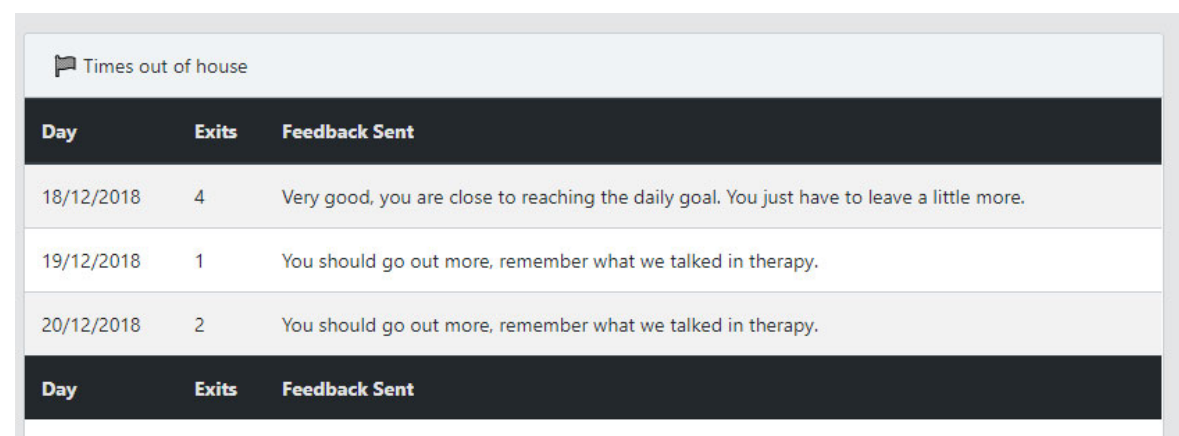

Figure 4.4: Screenshot of the visual interface of the configuration "Times out of home", as part of the viewer tool.

\subsubsection{Technological infrastructure}

The whole platform has been materialized as a distributed system composed of applications, tools and services, which communicate with each other following the client-server architecture pattern. That architectural pattern was ideal to satisfy the functional requirements of the different users of the tools: a mobile application with passive data collection capabilities for the patients, a web application with an app builder and data viewer tools for therapists, and a shared data storage system and analytic services accessible from both applications.

The functional and technical architecture of the platform can be seen in Figure 4.5. In previous sections, we have described the bottom part of the figure (shadowed box), with which patients and therapists interact. Next, we describe the other components and services that, although they are not visible to the therapists and patients, take a pivotal role in the platform. 
Chapter 4. Development of a builder tool for customizing location-based, gamified apps for depression.

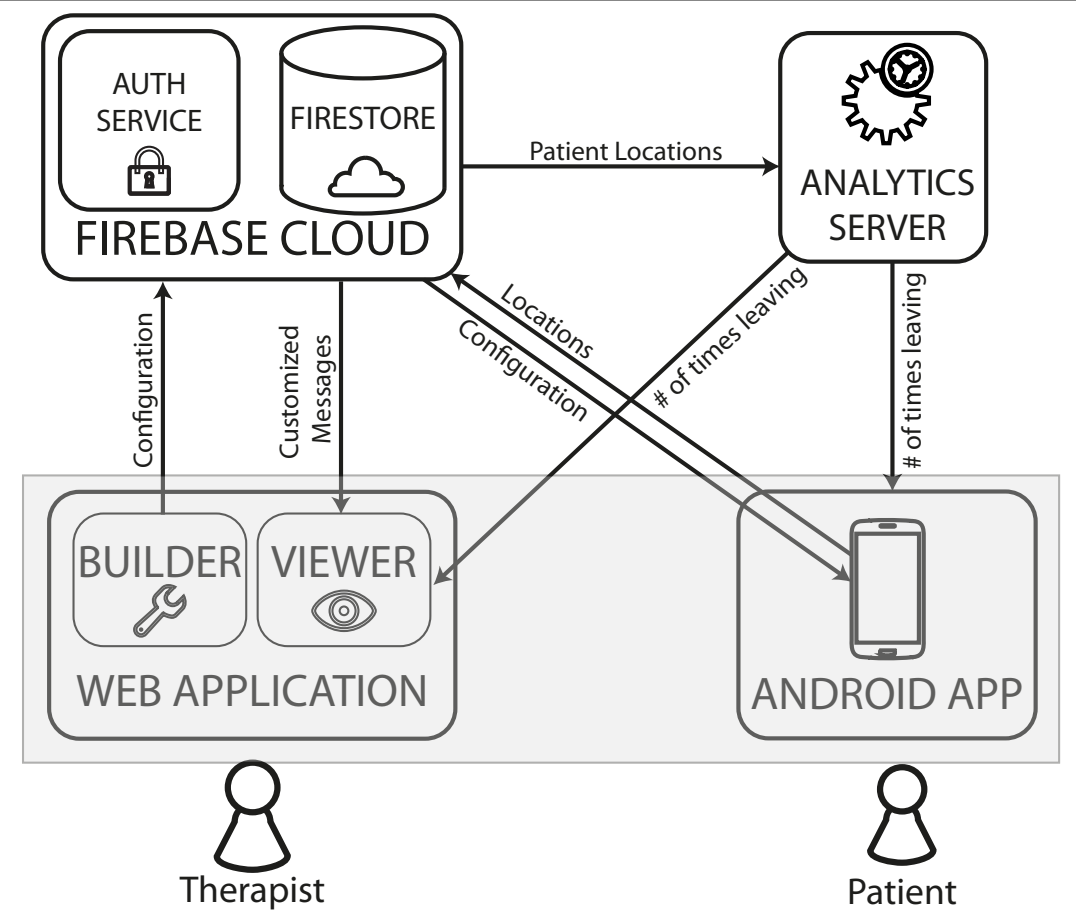

Figure 4.5: Functional architecture and main components, tools and services of the platform.

Web Application. It is the entry portal for therapists. It wraps the builder and the viewer tools, and hence provides unified access to both tools. When accessing to the builder, the therapists are allowed to add patients, configure mobile applications and assign patients to apps. When accessing the viewer, therapists can monitor patients, see their status and their interaction with the mobile application through the exploration of the collected data. Technically, the Web Application has been developed using widely-used Web technologies such as HTML5, TypeScript (JavaScript statically typed) and CSS technologies, and so the builder and the viewer. In particular, the Web Application is a single-page application (SPA) built with Angular framework [317] to make it highly modulable. The use of Angular allowed us to split the web application user interface (UI) into easy-to-manage, simpler components to ease the handling of business logic and the connection to external services. The $\mathrm{UI}$ is built upon the basic components of the CoreUl (Bootstrap-based) template [318] to achieve consistent aesthetic in all its views. One of the benefits of the Angular Framework is smooth compatibility with existing browser implementations. Therefore, the Web Application works in most modern browsers. 
As regards the interactions with other services and components of the platform, the builder authenticates users against a server-side authentication service, and stores the configuration file in a remote cloud database. To monitor patient's data, the viewer reads customized messages (as part of the configuration) from the cloud database, and also queries pertinent analysis services, as for example to compute the number of times a patient leaves home a day.

Android App. It is the generated mobile application used by the patient (section 4.3.2). In terms of the technology used and the data flow, the Android App collects location data passively, in a completely transparent manner for the patient. It is important to note that the patient never provides personal data but an access code generated by the therapist (using in the builder) which serves as anonymous user identifier. It also provides daily feedback through a notification, feedback that can also be obtained at anytime via opening the application. Strict rules in the handling of patient data are followed to ensure the privacy and security of personal data [319].

The app has been developed using Java and is compatible with the Android versions from 4.4 (KitKat) to 9 (Pie). For collecting user location it uses Google Play Location Services [320]. These services enable a higher location accuracy and less battery consumption, thanks to the intelligent use of a combination of satellite, WIFI fingerprints, cell phone and embedded sensor signals. Patient location is collected every minute by a foreground service launched by a scheduled alarm trigger. Custom app configurations (about the kind of notifications to deliver, when, etc.) are stored both in the server side and locally in a device's local database (SQLite) to allow offline usage. Like the web application, it remotely interacts with the authentication service, remote cloud database and the analysis services.

Auth Service. Centralized authentication to all tools and components of the platform is provided though a third-party service. Google's Firebase Authentication is the service of choice because it provides dual authentication, email-based and anonymous-based, and integrates smoothly with the (Android-based) applications and tools of the platform.

Firestore database. Like authentication, a third-party document database centralizes remote data storage and persistence for all the platform's tools and components. Google's Firebase Cloud hosts the authentication service (Auth Service) and the database service called Firestore, which is a scalable, flexible and schema-less database for storing ad-hoc configuration documents. This 
Chapter 4. Development of a builder tool for customizing location-based, gamified apps for depression.

database service stores all the data generated by the tools. Therapists store data associated to interventions, patients' identifiers, and mobile app configurations; and the mobile apps store mainly data collected passively from the patient. Firestore is secured by the authentication service, which integrates perfectly because both services are part of the Google Cloud family.

Analytics Server. Analysis methods are required to analyze the patient's data gathered in real time. Nevertheless, the detailed description of the design and implementation of the analytics server and the analysis services deployed on top of it are out of scope of this chapter. To better understand the role of spatial analysis in the platform, the analytics server can be regarded as a catalog of spatiotemporal analysis services specifically designed and created for their integration in the mobile app and viewer tool. The services take advantage of the reactive nature of the cloud database (Firestore) to populate a geospatial database [321] that facilitates performing spatial queries on the data. The analysis services have been developed with Python using Pandas and GeoPandas libraries, and they implement a public REST-based API interfaces to client applications.

Among the list of available analysis services, the following two are relevant in the context of this chapter (see requirements in Section 4.2.2): patient's home calculation (inferred from collected coordinates) and daily times outside home computation. The former service, the computation of the patient's home location, could be unnecessary because the patient (or the therapists) could directly provide his home location. Nonetheless, this is subject to a series of risks that could cause the information obtained to be incorrect. This service takes two days of data (baseline period), clusters them to rank the most visited places, and identify the most promising place as home. The second service, the number of times a day a patient leaves home, could also be calculated without the need for dedicated services, however the agility that these technologies provide to realize spatial queries benefits and facilitates access to this information. The web application and the patient's mobile app can access the exposed analysis services in order to get the number of times he has left his home. 


\subsection{Discussion}

\subsubsection{Strengths of the builder}

The main objective of this research was the development of a platform to expand the catalogue of tools of psychologists to enable them to monitor and intervene in patients with depression. The combination of knowledge and techniques from the fields of mental health, psychology, geoinformatics, and computer science required an important interdisciplinary effort for knowledge elicitation and integration. On one hand, we successfully established prolonged communication channels between the mental health practitioners and researchers, on one hand, and computer scientists and technicians, on the other hand, to understand therapists' domain-specific terminology and way of working. We carried out diverse research methods such as focus groups, interviews, and literature review to contrast the elicited information from various sources instead of using one single source. We also captured the technological and functional needs of the mental health practitioners and turned them into operational tools and applications. Indeed, the resulting information and insights go beyond the current development of the platform itself, expanding the possibilities for interdisciplinary research and collaboration in the future.

The builder tool has been designed to provide flexible customization. Each patient has different needs, so the builder allows the therapist to establish a custom app for each of them. The capacity of the therapists to quickly create apps adapted to the individual needs of patients is a fundamental step towards the achievement of personalized medicine in mental health [322].

From a technological point of view the platform has faced some challenges. The automatic detection of the patient's home represents an advance in the therapist's interaction with the user, reducing the errors that could arise from collecting it manually. We suffered some technical problems related to energy management and battery drained in the Android OS due to the continuous tasks of data collection and monitoring. We mitigated this issue by employing techniques that do not utilize only GPS measures, but take advantage of WIFI or accelerometer signals, reducing the number of requests to the GPS sensor to the strictly necessary. However, there is still room to optimize the data collection algorithms to obtain better accuracy and consume less energy. 
Chapter 4. Development of a builder tool for customizing location-based, gamified apps for depression.

\subsubsection{Limitations and future perspectives}

Although gamification is a part of our design, its use in this work is superficial, limited to motivation techniques (textual messages, progress bar) to improve patient adherence. However, it presents a starting point, since techniques such as achievements, grant points, unlock content and new phases within the treatment are interesting improvements that could be supported by next releases of the platform. Allowing the therapist to adapt the treatment by unblocking new psychotherapeutic content as patients reach certain milestones in the treatment is a promising gamification strategy viable through the builder tool. Finally, the selection and inclusion of gamified elements in the user interface can be driven by patient profiles. For example, more childish designs with avatars for clinicians working with children, or more formal or serene interfaces for adult patients.

Technical issues prevented us from delivering the platform for real-work experimentation as planned. Nevertheless, the ultimate goal is to reach a number of patients to statistically validate the usefulness of the entire platform. Future plans necessarily includes to test with real patients through case studies or $n=1$ trials to later move to more robust designs such as RCT. Such a gradual experimentation would allow us to $1 /$ evaluate the satisfaction of the patient and therapist, 2/ to assess the effectiveness of the behavioral activation component by using the mobile application and, $3 /$ to better understand to what extent the information received by the therapist in real time allows to modify and adapt the treatment.

\subsubsection{Conclusion}

This work presents the design and technical development of a platform to assist therapists to create and personalize location-based gamified mobile applications for depression treatments. The Requirements elicitation through meetings and focus group with psychologists has been described, as well as the process of design and development of the entire platform and associated tools. In summary, the lessons learned during the development phases and early tests of the platform with therapists open a door to endless possibilities for extensions and applications: to address mental diseases other than depression, to add new gamification techniques to increase patient adherence, to develop specific features for realtime monitoring and better-adapted visual designs to enhance patients' perceived 
satisfaction of mobile device-based interventions. 


\title{
Validation and experimentation of the platform in case studies with real patients.
}

\author{
The world is not in your books and \\ maps, it's out there!
}

Gandalf the grey.

This chapter presents the validation of the platform with three use cases in patients with two different disorders. Each disorder is treated in a different section. The first one describes the treatment carried out in a patient suffering from panic disorder and agoraphobia. In the second section, the treatment for two patients with gambling disorder is explained. Both sections present a similar structure to present the case studies and the use of the platform developed in the framework of this thesis. 


\subsection{Enhancing in vivo exposure in the treatment of panic disorder and agoraphobia using location- based technologies: A case study.}

Panic disorder (PD) is a prevalent disorder in the general population, being especially common with agoraphobia (PD/A). The treatment of choice for PD/A is Cognitive-Behavioral Therapy (CBT), which is considered a first-line treatment. New developments in psychopathology have increased the emphasis on the common underlying mechanisms that contribute to the development and maintenance of emotional disorders such as PD/A. This is the case of transdiagnostic intervention, an emotion-focused, cognitive behavioral intervention which has led to the Unified Protocol (UP) as a form of CBT therapy to help patients learn new ways of responding to their emotions. A core feature of this treatment approach is the in vivo exposure (IVE) to feared situations which aims to prevent avoidance behaviors and confront the patient with the feared situations gradually, with the goal of habituation. However, it is a hard component for patients, especially when implementing the exposure on their own, which leads to inherent difficulties of exposure for those people who suffer PD/A. To help the patient, different formats of feedback can be used to increase adequate IVE and diminish overt or subtle avoidance. Particularly, Information and communication technologies (ICT) and specifically the use of smartphones can be a very useful option to initiate and sustain the exposure behavior. The purpose of the current case study is to describe the use of location-based technologies (LBT) when using a smartphone app during the IVE component in the treatment of a 47-year-old patient with a diagnosis of severe PD/A. In addition, the acceptability and usability of the system was assessed by the patient. The patient received the UP for Transdiagnostic Treatment of Emotional Disorders delivered by the UP Institute, Center for Anxiety and Related Disorders (CARD), Boston University. The ICT platform Symptoms was used during the situational exposure module, using LBT with a smartphone app. The results show that the use of LBT presents relevant implications for the treatment of $\mathrm{PD} / \mathrm{A}$ and suggest several positive consequences of monitoring the IVE sessions in real time during the patient's treatment. In addition, studying the patient's acceptance and satisfaction regarding the use of the technology was considered an important aspect. The patient reported positive expectations and 
Chapter 5. Validation and experimentation of the platform in case studies with real patients.

high satisfaction scores, and an overall satisfactory experience with the platform. Enhancing key therapeutic components during psychological treatment through the development of media-based tools constitutes very promising future research aims and new possibilities, that arise especially from advanced smartphones' features, should be explored.

\subsubsection{Introduction}

The Diagnostic and Statistical Manual of Mental Disorder-5 (DSM-5 [55] associates panic disorder (PD) to recurrent and unexpected panic attacks followed by worries about their consequences concerning physical or social aspects [55]. The 12-month prevalence estimated for panic disorder in adults and adolescents across the United States and several European countries is around 2\%-3\% [323]. Regarding panic disorder, the prevalence is especially common together with agoraphobia disorder, with agoraphobic symptoms estimated to affect between $33 \%$ and $50 \%$ of patients with PD [324]. Agoraphobia disorder is described as experiencing a marked fear or anxiety triggered by real or anticipated exposure to a wide range of situations [55].

These disorders are frequently associated with a high level of social impact (avoidance of relevant personal events), occupational impairment (absent from work or school), and physical disability (comorbid with numerous general medical conditions). In addition, PD entails a considerable socio-economic cost and records the highest number of medical visits of all anxiety disorders, being the effects strongest with the presence of agoraphobia. These symptoms, which are linked to the diagnosis, are clearly associated with a poorer quality of life [55].

The treatment of choice for panic disorder and agoraphobia (PD/A) is CognitiveBehavioral Therapy (CBT) which is considered a first-line treatment [325-330]. The efficacy of this treatment is supported by The Task Force Reports on Empirically Supported Treatments (EST) [331] and the 12th Division of the APA. It has been further reinforced by meta-analysis efficacy studies that report finding important and long-lasting improvements [332, 333]. The core feature of CBT therapy is the in vivo exposure (IVE) to feared situations which aims to prevent avoidance behaviors and confront the patient with the feared situations gradually with the goal of habituation [334]. This component is well established and is used to disconfirm misappraisals and eliminate conditioned emotional responses to external situations 
5.1. Enhancing in vivo exposure in the treatment of panic disorder and 100 agoraphobia using location-based technologies: A case study.

in agoraphobic situations linked to the disorder. In spite of the efficacy of CBT and IVE, it is a hard component for patients, especially when implementing the exposure on their own. This leads to inherent difficulties of exposure for those people who suffer PD/A [335, 336]. It, therefore, becomes important to enhance IVE and its motivational features by some kind of therapeutic support.

Traditionally, guidelines for IVE sessions have included the support of a cotherapist during the exposure or even the presence of the therapist outside of the therapy office [337]. In addition, to help patients, different formats of feedback have also been used to increase adequate IVE and diminish overt or subtle avoidance [338]. However, some of these measures can have potential costs, such as time and interference, with patients turning these measures into safety signals [337]. Moving forward, new developments in psychopathology have increased the emphasis on the common underlying mechanisms that contribute to the development and maintenance of multiple disorders, especially emotional disorders (as the case of PD/A) and are designing novel strategies to target these disorders [339]. This is the case of transdiagnostic intervention, an emotion-focused, cognitive behavioral intervention which has led to the Unified Protocol (UP) as a form of CBT to help patients learn new ways of responding to their emotions [340]. A number of studies [341-346] including important randomized controlled studies (RCT), have shown that the transdiagnostic approach and the UP can be as effective as the CBT traditional diagnostic approach [340]. In addition, reviews and meta-analysis studies have reported promising results [347-349].

In recent years, there has been an increased interest in using technologyassociated psychological interventions as a form of treatment for emotional disorders, including PD/A. Several studies have been conducted in order to improve the effectiveness of psychological treatments or clinical utility. The first promising technology was virtual reality (VR), which emerged as an effective tool for applying IVE, reporting efficacy in the treatment of anxiety disorders [7-9], and more specifically of PD/A [10,11, 13,350]. These pioneer studies included the CBT approach. Furthermore, media development has led to the use of the Internet to deliver CBT, obtaining very adequate results by RCT [14], systematic review and meta-analysis studies in which the efficacy of these computerized programs has been confirmed [15-17, 351]. Additionally, new approaches, such as the previously mentioned transdiagnostic intervention, have been tested as an effective treatment option in a web-based format for affective and mood disorders by RCT studies 
Chapter 5. Validation and experimentation of the platform in case studies with real patients.

[351-353].

The implementation of Information and Communication Technologies (ICT) for psychological intervention has been empirically tested. Along with the technology itself and the clinical effectiveness, the issue of acceptability of the technologyguided treatment is important when considering whether or not the use of ICT will affect the therapeutic adherence and outcomes [354]. Acceptability refers to the degree to which users are satisfied or at ease with the service and willing to use it [355] and influences whether a treatment is perceived as fair and reasonable, appropriate, and non-intrusive in addressing a problem [356, 357]. In the Technology Appraisal Guidance from United Kingdom, treatments are considered a priority to properly assess intervention acceptability and the variables linked to it; expectations, satisfaction, and usability. This in turn, makes these variables become crucial features in psychotherapy results [358]. Several studies have focused on the expectations and satisfaction with different ICT tools such as VR or Internet-based treatment for delivering psychological treatments and specifically the IVE in mental disorders and PD/A [10, 359, 360], reporting high satisfaction with the exposure component mediated by technologies. Studying acceptability and usability of an ICT has to take into account a very specific conceptualization inside the user experience, exploring the ease of use of a product by a specific user with clearly defined context and goals with the aim of enhancing the ICT development. Even though few studies have addressed this topic [357, 361, 362], the before mentioned ICTs have shown to be well-supported regarding acceptability issues.

In mental health, additional advantages can be added by another ICT such as mobile phones and smartphones. Smartphone utilization has experienced a huge increase over the last years [18] and in consequence, researchers have started focusing on the impact of these devices when used as tools to implement psychological intervention and IVE components [363]. Smartphone portability could be very useful in a variety of feared situations in which PD/A symptoms happen. Additional smartphone features such as location-based technologies with personalized feedback of the patient's position could signify a starting point to initiate and sustain the exposure behavior. In consequence, it can lead to the improvement of IVE through the possibility of maximizing the motivation and commitment of the patient. Although LBT could become a tool when prescribing IVE, it will be upon the patient to carry out the exposure on his/her own. This study 
5.1. Enhancing in vivo exposure in the treatment of panic disorder and 102 agoraphobia using location-based technologies: A case study.

assumed a set of advantages of using these devices: a) the therapist can ensure that the patient actually is in the exposure situation; b) the therapist can ensure that the patient stays in the exposure situation for the necessary time in order to fulfill the goals; and c) the patient's motivation and commitment can be enhanced in the real exposure environment. In previous works, when carrying out the treatment, the context has been considered and treated as a variable. In the study of Addepally et. all [124], they looked at using a mobile application (app) that would monitor the location of depressed people. The app detected if the depressed patients were in less-crowded areas (a frequent trait in depressed individuals according to the authors) and, if affirmative, the patient would receive therapeutic strategies and self-help assessment through the use of notifications. In a case report study [364], a patient suffering from obsessive-compulsive disorder was allowed to configure alarms in case they remained for a long time without changing places, using an app that tracked their position. However, to the best of our knowledge, there are no reports that account for LBT in a smartphone application for the treatment of $\mathrm{PD} / \mathrm{A}$ disorder during IVE. With all this in mind, the purpose of the current case study is to describe the use of LBT in an IVE component during the treatment of a patient diagnosed with severe PD/A while assessing the acceptability and usability of the system by the patient. The hypothesis for this study was that the location-based technology would enhance IVE during the treatment of the PD/A by a) enhancing motivation and patient commitment b) reducing the avoidance in a disorder in which location is core and c) being well-accepted by the patient.

\subsubsection{Method}

\section{Patient}

The patient is a 47-year-old married male who worked as a sport trainer and met the Diagnostic and Statistical Manual of Mental Disorders, fifth edition (DSM-5) criteria for panic and agoraphobia disorder. He has previously received treatment for this problem but discontinued it because he did not perceive any improvement. Although he does not remember exactly when his problem began, he reports having the problem since he was a child, probably starting with a fear of heights that evolved to other situations. The problem became progressively worse, to a point of having a severe interference in his life, until he sought out help in our clinic. At that time, he rated his problem's severity and interference both as 8 
Chapter 5. Validation and experimentation of the platform in case studies with real patients.

(in a scale ranging from 0 to 10 ). The main physical sensations experimented by the patient included hand sweating and dizziness. The patient stated being extremely concerned about the possibility of suffering panic attacks in a feared situation which could onset the arrival of physical symptoms that could result in loss of control. This problem has additionally created issues in his professional life because he feels he cannot move far away from his town. Due to his problem, several of his job tasks have been impaired resulting in him losing central work activities. In terms of medication, he has been taking $1 \mathrm{mg}$ of alprazolam daily during years and reports that it helps him sleep well. When the patient arrived at our clinic, he arrived with significant agoraphobic avoidance even though he did not experience unexpected full panic attacks now because he avoided every feared situation. The patient presented comorbidity with acrophobia.

\section{Therapist}

The therapist was a member of our research team with more than ten years of experience in the therapeutic field including both technology-mediated and psychological intervention.

\section{Measures}

Several common psychiatric testing measures were used in order to establish the diagnosis and to evaluate the effects of the intervention. However, given that the component under study was IVE by location-based technologies (IVE+LBT), only the measures related to this component will be presented in the chapter (with the exception of the diagnosis measure).

Diagnosis measures and measures for the target behaviors:

- Anxiety Disorders Interview Schedule-IV [365] (ADIS-IV). The ADIS- IV is a semi-structured interview that assesses anxiety disorders included in the DSM. The ADIS-IV has demonstrated interrater reliability from satisfactory to excellent when used by expert clinicians who are familiar with the DSM diagnostic criteria [365]. In the present study, the PD and agoraphobia and specific phobia sections were used.

- Fear and avoidance scales (adapted from Marks and Mathews [366]. The patient and the therapist established target behaviors or situations that the 
5.1. Enhancing in vivo exposure in the treatment of panic disorder and agoraphobia using location-based technologies: A case study.

patient avoided because of his PD/A. The target behaviors were defined as the behaviors for which avoidance created substantial impairment in the patient's daily life. He rated the level of avoidance on a scale of $0-10(0=1$ never avoid it; $10=I$ always avoid it) and the level of fear on a scale of $0-10$ $(0=$ no fear; $10=$ extreme fear) for each target situation.

Measures for expectations and satisfaction with the ICT system:

- Expectation and satisfaction scale (adapted from Borkovec and Nau [367] regarding the IVE+LBT component used previously in our research group in other ICT contexts [359, 368, 369]. This questionnaire was used to measure patient's expectations, before, and satisfaction, after, the IVE+LBT component. The questionnaire included six items; how logical the IVE+LBT component seemed, to what extent it could satisfy the patient, whether the patient would recommend this component treatment to others, whether it would be useful in treating other problems, the component's usefulness for the patient's problem and to what extent it could be invasive. This last item was considered a key factor in assessing the ICT system during IVE. Due to the main characteristics of the LBT, it was important to assess any disruption, annoyance or intrusion on patient's privacy caused by the ICT. Both parts (expectations and satisfaction) ranged from 0 to 10 , being $0=$ "not at all" and $10=$ "very much".

Measures for acceptability and usability of the ICT system:

- System Usability Scale [370] is one of the most used tools for assessing perceived usability [371]. It consists of ten items, half of them are written in a direct style and the other half in an inverse style. A five-point scale is used for rating the level of agreement, from 1 (strongly disagree) to 5 (strongly agree). This scale has a score contribution of the scale position minus 1 for the items 1,3,5,7 and 9, and 5 minus the scale position for the items 2,4,6,8 and 10. A formula is used to calculate the score as a percentage scale from 0 to 100 .

\section{Treatment and IVE}

The patient received the UP for Transdiagnostic Treatment of Emotional Disorders delivered by the UP Institute, Center for Anxiety and Related Disorders 
Chapter 5. Validation and experimentation of the platform in case studies with real patients. 105

(CARD), Boston University. The treatment program was structured into 8 modules. The sessions included in each module lasted around 1 hour and were delivered weekly. The IVE+LBT component was carried out during the interoceptive and situational exposure module, keeping in line with the structure of the UP program [372]. The patient was exposed to his main target behaviors in a gradual exposure hierarchy, progressing from the easiest to the most challenging situations and following the exposure hierarchy created between the patient and the therapist. LBT was used during the IVE.

\section{System description}

The application used with the case study was developed in 2018 as part of an ongoing platform called Symptoms. The goal of the Symptoms platform is to create personalized, location-based mobile applications for mental health treatments. The platform presents three different tools: a web-based Builder tool, the generated mobile APP, and a web-based Viewer tool:

\section{Builder}

The therapist accesses the web-based Builder through a URL using widely supported web browsers. Once registered and identified, the therapist can create patient profiles and applications. To create a patient profile, the therapist introduces some basic descriptive data (Figure 5.1). To create an application, the therapist must enter some basic data and then s/he has to select on a map the relevant places for the patient. In addition, for each of these places, s/he has to enter a personalized message (Figure 5.2). Once the patient and the application have been created, the therapist links them both, so that the application is assigned to the patient. In technical terms, this personal link is ensured by generating a unique Application Key (AppKey), which must be used to activate the mobile application. The builder has additional, configurable characteristics which allow the therapist to handle diverse situations with ease. For example, as the treatment evolves, so does the application but the patient only needs to upgrade her/his mobile-app as usual. Another example is that instead of having one-to-one relationships (one application, one patient), the same application can be assigned to a group of patients with similar conditions. 
5.1. Enhancing in vivo exposure in the treatment of panic disorder and 106 agoraphobia using location-based technologies: A case study.

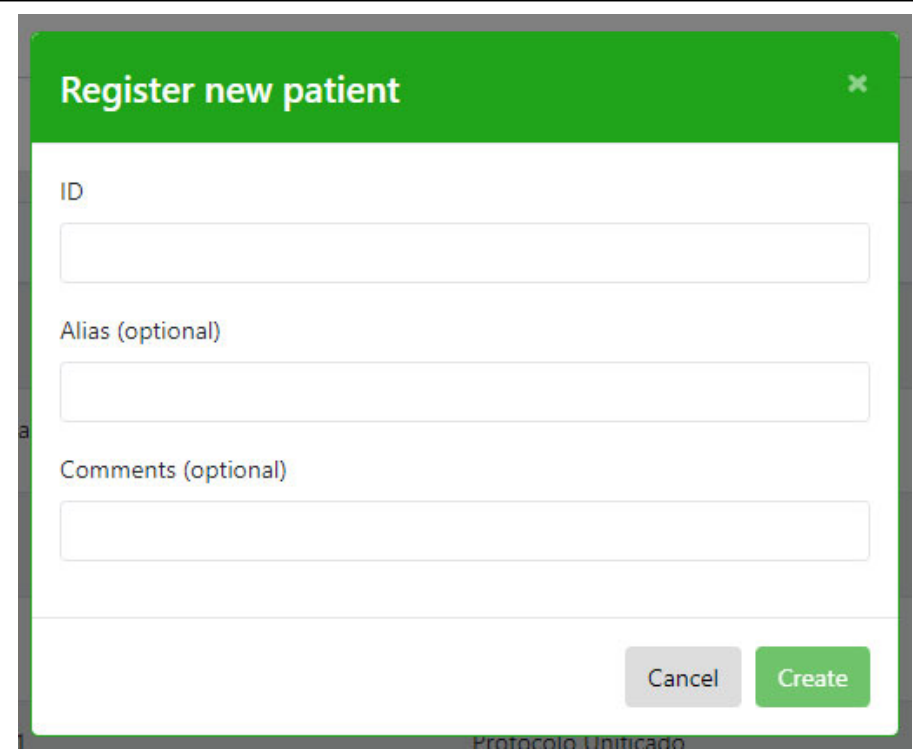

Figure 5.1: Screenshot of the Create Patient form.

\section{App}

The outcome of the builder tool is an Android-based mobile application. The patient downloads it on her/his mobile phone from the Google Play Store and installs it. To launch it the first time, an AppKey, which is provided by the therapist, is requested (Figure 5.3). Once the AppKey is entered, the mobile application is unlocked and starts working in background mode, without interfering with the patient's regular use of the smartphone. Once the app is running, every time the patient arrives at any of the places that the therapist has configured, $s / h e$ will receive a notification with the personalized message that the therapist had written for that particular place.

\section{Viewer}

The Viewer and the Builder are two sides of the same coin. The therapist can access the web-based Viewer through the same URL as the Builder. Therefore, the builder and the viewer complement each other. Therapists can only access the data produced by their applications, i.e., those created by them using the builder. Once authenticated, a therapist can browse and analyze the patient's gathered data. S/He can also inspect the gathered data according to the spatial and temporal dimensions, by observing the places visited by the patient (Figure 5.4). 
Chapter 5. Validation and experimentation of the platform in case studies with real patients.

\section{New Place}

Place Name

High Bridge

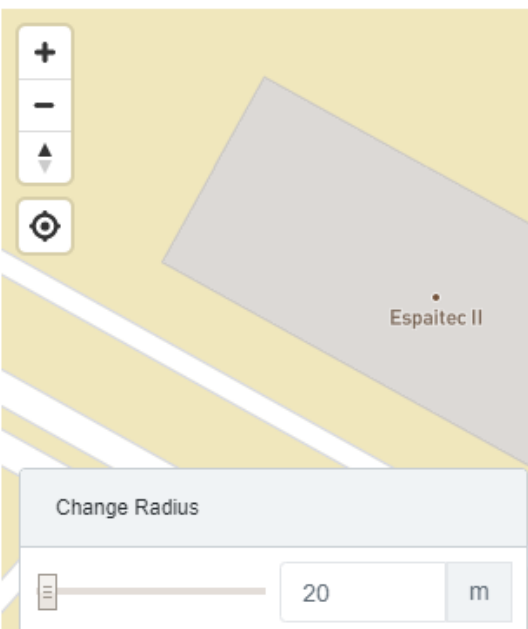

(2) meaposs Associated Message

You have reached one of the bridges you fear most. Remember what we talked about.

Figure 5.2: Screenshot of the New Place form. 
5.1. Enhancing in vivo exposure in the treatment of panic disorder and agoraphobia using location-based technologies: A case study.

\section{$\Downarrow \xi$ ว $4100 \%$ 10:28}

\section{Symptoms}

\section{Insert an application key.}

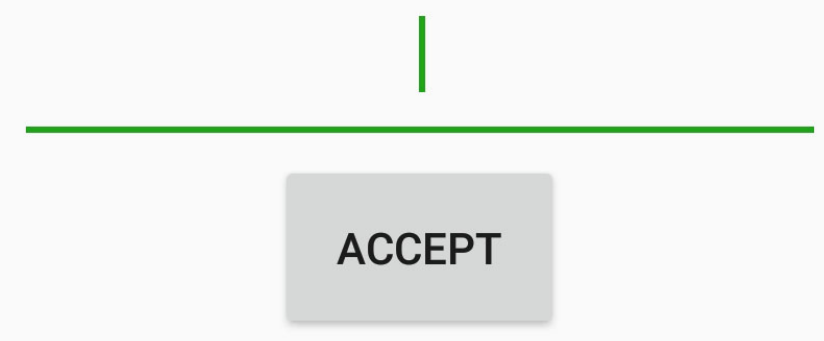

Figure 5.3: AppKey Request Screenshot.

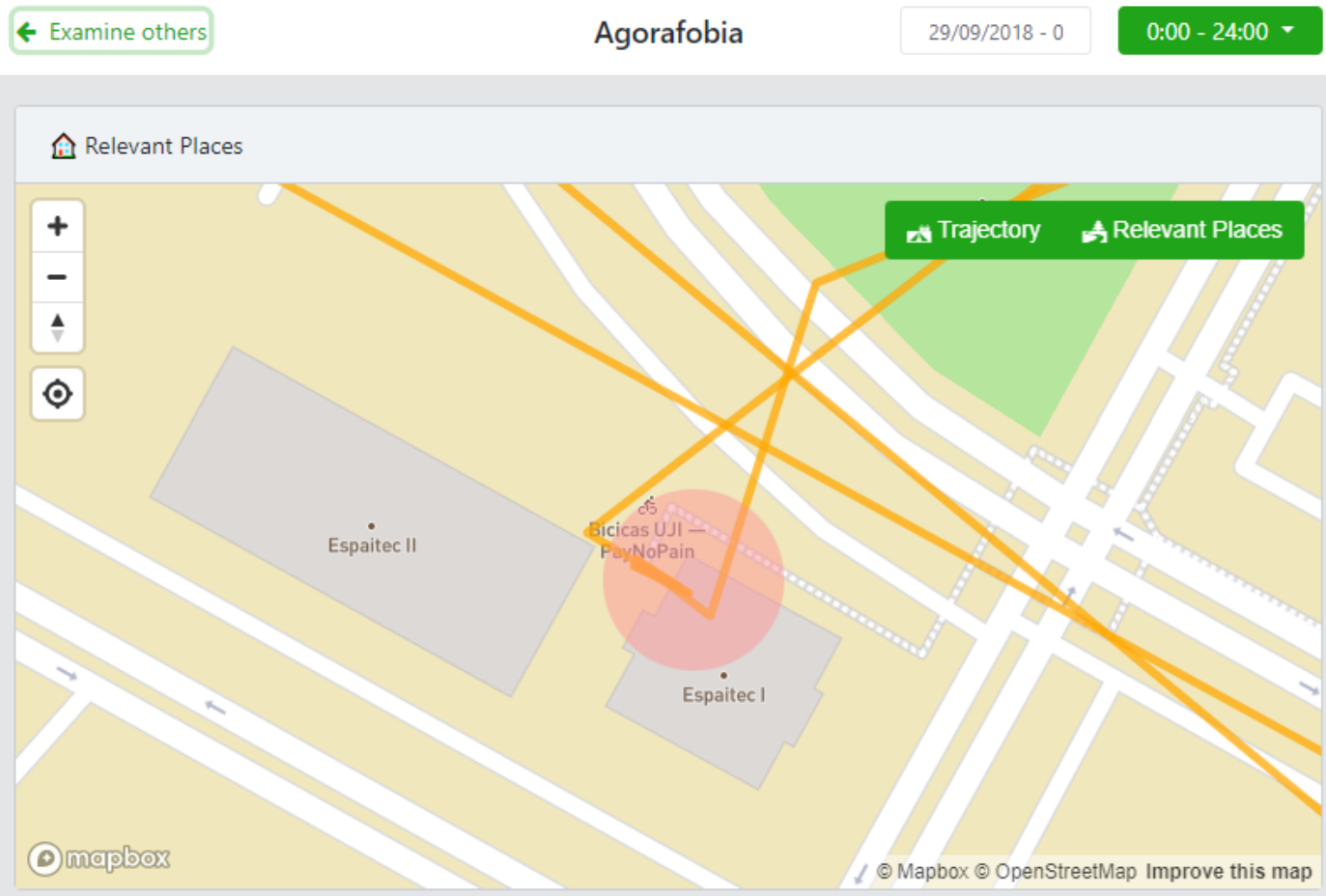

Figure 5.4: Screenshot of the viewer. 
Chapter 5. Validation and experimentation of the platform in case studies with real patients.

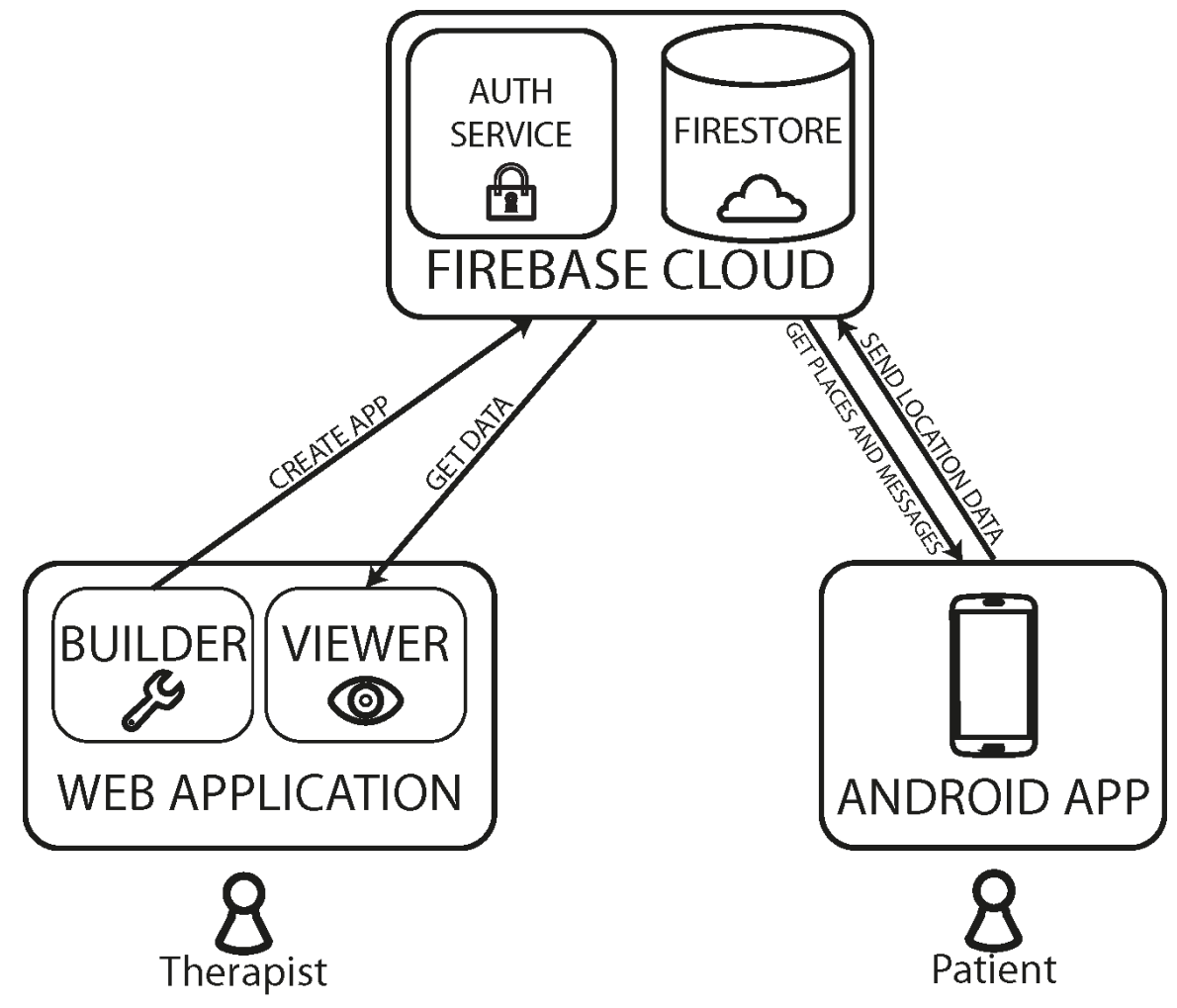

Figure 5.5: Technological Architecture of the Symptoms Platform.

The whole platform is developed by integrating a mix of technologies. Figure 5.5 depicts the architecture of the platform. The Builder and the Viewer are part of the same web application, developed with the JavaScript Angular framework version 6. They are connected to a Google Firebase's Firestore database, for data persistence, and both utilize the Firebase's authentication system. The Builder's database stores the patient's data and the applications' configuration data, including selected places and personalized messages. Using the Firebase Functions, the Builder lets therapists associate an application with a patient by automatically generating an AppKey.

The mobile application is developed in Java using the Android Studio development environment. As the app connects to the same database as the Builder and Viewer, it can download the important places with their associated messages. The AppKey is used to ensure that the correct configuration is loaded into the app. Once the app is correctly initialized, it runs a background service for patient monitoring and data gathering. This service uses Google's location-based services to gain access and communicate with different embedded sensors such as 
5.1. Enhancing in vivo exposure in the treatment of panic disorder and agoraphobia using location-based technologies: A case study.

accelerometer, GPS or Wi-Fi. In this way, the current location of the patient is captured. The requesting interval is not fixed. During the night, or whenever the mobile device is motionless, the app will detect this lack of movement through the accelerometer, and the time between requests will increase. By doing so, the app saves battery. When a new location is received and stored, it is checked against the list of stored places. If the user is in one of the stored place, a notification with the message of the therapist is sent to the user. The user's location is stored both locally and in the Firestore dateabase.

Finally, the Viewer connects to the Firestore to access the patient's information and allow the therapist to explore it through tables, charts, and maps, offering different viewing options (by dates, by place, aggregated, etc.).

\section{Design and procedure}

This study follows a case study design. The patient asked for help at the Jaume I University Anxiety Disorders Clinic, Spain. First, he underwent a screening assessment and, having met the inclusion criteria, he then signed a consent form to participate in the study. Inclusion criteria included the following: a) meeting the panic and agoraphobia diagnostic criteria and b) providing written, informed consent. Exclusion criteria included: a) suffering from a severe mental disorder (schizophrenia, bipolar disorder, and alcohol and/or substance dependence disorder); b) medical disease/condition that prevents the participant from carrying out the psychological treatment; or c) receiving another psychological treatment during the study. The assessment consisted of two 60-minute sessions to evaluate the diagnosis and establish the target behaviors. The ADIS-IV interview was carried out in the first session and the second session was used to conduct other self-report measures and establish the patient's target behaviors. The rest of the measures linked to the UP treatment were carried out in the context of the full therapy. Given that the component under study was the IVE+LBT, only the measures related to this component will be presented in the chapter and emphasized in this procedure section. Following the assessment and before starting the treatment, the therapist explained the basis of the treatment and the use of LBT to support the IVE. The patient agreed to take part in the research.

Once the consent form for participating in the research and the consent form to use the LBT were filled out properly, the treatment started. The exposure 
Chapter 5. Validation and experimentation of the platform in case studies with real patients.

sessions were conducted in correspondence with the UP modules. Before starting the exposure module, therapist and patient created a gradual exposure hierarchy attending to the core situations feared by the patient. Then, the expectation about IVE+LBT component was evaluated by the patient before the first exposure task was conducted. The locations assessed by the fear and avoidance scales were configured and their positions were set by the therapist and the app was installed on his own smartphone. Every time the patient arrived at one of the core places during the IVE tasks, he received a notification with the particular message written by the therapist "you have just arrived at an important place. Remember to work with all the strategies you know". The LBT was used during the patient's IVE tasks, in which he had to confront the different scenarios related to his fear. Following all the exposure tasks, the satisfaction and acceptability and usability of the IVE+LBT were measured by the patient with the self-report measures.

\subsubsection{Results}

\section{Target Behaviors}

The patient carried out weekly IVE for a period of 4 months, in different situations. Figure 5.6 displays the main target behaviors selected for the exposure tasks, as well as the fear and avoidance ratings from the patient. As shown in the figure, they were bridge, mountain, train, and music concert. All the selected target behaviors caused a certain level of impairment in different areas of the patient's life.

The locations were positioned by the LBT and configured to receive the notification when the patient arrived at these core places during exposure tasks. Once the exposure session started, the patient achieved $100 \%$ of the goals established regarding the exposure tasks, that is, there was no avoidance in any case at the end of the exposure session. Even though it was not a goal of the present study, preliminary results regarding the fear and avoidance situations show an important reduction of the score of the scheduled target behaviors rated by the patient and positioned by the IVE+LBT.

\section{Expectations and Satisfaction regarding the IVE+LBT}

Table 5.1 shows the patient's acceptance of the treatment. He reported high expectations before starting the IVE+LBT component. In addition, he made a 
5.1. Enhancing in vivo exposure in the treatment of panic disorder and agoraphobia using location-based technologies: A case study.
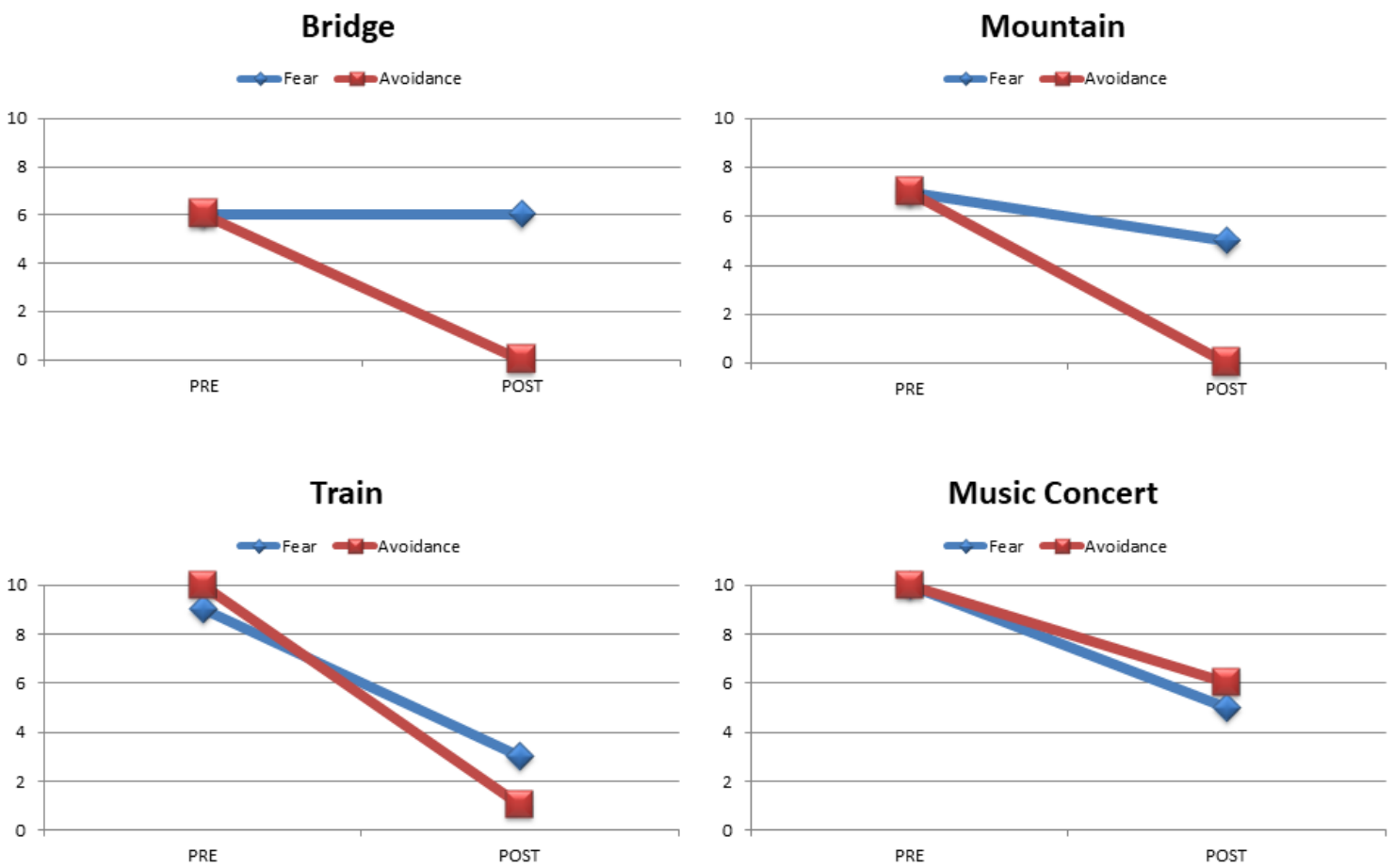

Figure 5.6: Located target behaviors with fear and avoidance rating.

highly positive evaluation afterward. Especially important for this research is the question about to what extent it could be invasive. The patient rated that question as "not at all". The loss of privacy due to detailed information about the system usage being sent to the therapist could have created interference with the opinion about the system. However, this was not the case in this study.

\section{Satisfaction and usability of the IVE+LBT}

The perceived satisfaction and usability for the user was very high in all items except item 4 (see Table 5.2). This item refers to the following statement "I think that I would need the support of a technical person to be able to use this system". An interview with the user revealed that this score was due to technical problems experienced one day during exposure tasks. This problem was related to the frequency with which the mobile phone made the GPS request. The Android 
Chapter 5. Validation and experimentation of the platform in case studies with real patients.

Table 5.1: Expectations and satisfaction with IVE+LBT.

\begin{tabular}{lcc} 
& Expectaction IVE+LBT & Satisfaction IVE+LBT \\
\hline Item 1 & 10 & 10 \\
\hline Item 2 & 10 & 10 \\
\hline Item 3 & 10 & 10 \\
\hline Item 4 & 10 & 10 \\
\hline Item 5 & 10 & 10 \\
\hline Item 6 & 0 & 0 \\
\hline
\end{tabular}

operating system has some particular policies in the use of background services, this happens because of an issue of energy management. By default, the system slows down high consumption processes to reduce battery consumption and in some cases, this can delay the Geolocation service of the app, causing a delay in message delivery. Once the problem was detected, the background service was partially modified to improve its execution precision (making the application request energy management permissions at the beginning of its use). With these changes, the messages worked properly again.

Table 5.2: Usability test about IVE+LBT.

\begin{tabular}{lc}
\hline Item 1: I think that I would like to use this system frequently & 5 \\
\hline Item 2: I found the system unnecessarily complex & 1 \\
\hline Item 3: I thought the system was easy to use & 5 \\
\hline $\begin{array}{l}\text { Item 4: I think that I would need the support of } \\
\text { a technical person to be able to use this system }\end{array}$ & 3 \\
\hline Item 5: I found the various functions in this system were well integrated & 5 \\
\hline Item 6: I thought there was too much inconsistency in this system & 1 \\
\hline Item 7: I would imagine that most people would learn to use & 5 \\
this system very quickly & 1 \\
\hline Item 8: I found the system very cumbersome to use & 5 \\
\hline Item 9: I felt very confident using the system & 1 \\
\hline Item 10: I needed to learn a lot of things before I could & \\
\hline get going with this system &
\end{tabular}

The overall value for satisfaction and usability was 95 points, which according to 
5.1. Enhancing in vivo exposure in the treatment of panic disorder and agoraphobia using location-based technologies: A case study.

the qualitative scale developed by Bangor et al. [373] means that the system is within an acceptable range, with adjectives rating between "excellent" and "best imaginable".

Finally, some qualitative information was collected from the patient's experience, here are some of the opinions expressed by the user after the IVE+LBT component:

General opinion:

"The use of the system was very interesting for me, especially in the motivational aspect. It was extremely important for me to have a record of the completed exposures".

Specific experiences:

"There was a day in which I forgot the smartphone with the app, and I went back in order to carry out the exposure task. The most important aspect about the system, one which I really appreciated, was the role it took on of a personal trainer for each exposure task".

Regarding the notification:

"Ok, the notification could be interesting, but it was not the most important thing for me. To record my activity and to record the work well done, that was what made me even more committed throughout the treatment".

\subsubsection{Discussion}

The results obtained in this case study show that the use of LBT presents relevant implications for the treatment of $\mathrm{PD} / \mathrm{A}$. The positive consequences of monitoring the IVE sessions in real time during the patient's treatment seem to be several; 1) the certainty that the patient's efforts are being recorded and visualized by the therapist 2) the technology is well-accepted by the patient and 3) it has the possibility of increasing the adherence and commitment to the scheduled IVE tasks. The patient reported positive expectations and high satisfaction scores, coinciding with previous studies showing that ICT treatments are well accepted $[10,359,360]$. These results with more advanced technology reach a high importance, since a positive relation between expectations and satisfaction with the ICT treatments and intervention efficacy have been found in [374]. In addition, the data on intrusiveness are especially relevant. As pointed out earlier, the patient reported that the IVE+LBT was not invasive before and after the treatment and that it did not interfere with the fulfillment of the exposure tasks. Finally, usability 
Chapter 5. Validation and experimentation of the platform in case studies with real patients.

results reported a satisfactory experience of the patient with the system. Based on the technology acceptance model, the authors have suggested that one of the factors that can be related to the intention to use a product in the future is ease of use $[183,188]$. Therefore, the usability, which is already an important attribute in the use of any technology [375], becomes a prerequisite in the use of technology for mental health. Technology must be completely transparent from the beginning, otherwise, a slow learning curve or high frustration during use would interfere with the therapy and negatively influence the outcome of the treatment. Therefore, efforts to research and ensure the usability of new ICT approaches are crucial.

Previous research has focused on the ethical considerations of using exposure therapy [335] and how it may become problematic in different stages of the therapy outside of the safety context with therapist or co-therapist. The development of specific ICT strategies as additional tools to guide the IVE can be useful in reducing avoidance, increasing overall functioning, and enhancing the motivation and commitment of the patient with critical components of the therapy, and in this case, feared situations. In short, one could ask oneself if some of these tool-guided exposure elements could significantly improve adherence to treatment and reduce drop-out rates (a major issue encountered in research studies), particularly in $\mathrm{PD} / \mathrm{A}$ which is highly characterized by avoidance behaviors, attrition ranges from $10 \%$ to $55 \%[354,376,377]$ and where some variables of the disorder can affect the treatment's discontinuation.

From a technical point of view and given the simplicity of the creation of the application, the adaptability of this tool is also presented as a positive feature. Changing the indicated places or the information delivered can be done fast and without requiring specific technical knowledge. This makes adapting the application to the evolution of the patient or to be used with others an easy task, bypassing the technological barrier that involves customizing applications for different patients, even for the therapists. Generating this type of in-situ intervention has always been somewhat complex, requiring time-consuming traditional methods or trusting the patient to carry out the indicated tasks. Thanks to this tool, this process has been streamlined, allowing the therapist to focus their attention on the therapeutic content and delegating the monitoring and delivery of the materials to the mobile device.

With regards to the application itself, at the moment it only allows for a basic configuration: places and information in text format. However, it would have been 
5.1. Enhancing in vivo exposure in the treatment of panic disorder and 116 agoraphobia using location-based technologies: A case study.

interesting to be able to include other variables, for example, different messages arriving depending on the time (a message at the beginning of the exposure, a message in the middle and one at the end) or more complex content such as multimedia. Currently, only text information is allowed but moving forward it would be interesting to include images or videos to improve the quality of the intervention. Although the application is focused on intervention, it would also have been positive to use real-time feedback in the form of questionnaires that are able to identify, for example, the anxiety of the patient. In general, there are a series of functions, which do not have a great complexity from the technological point of view, which would allow to considerably improve the options when creating the application. In terms of the study's shortcoming, the main one is that it is a case study. In order to increase the confidence in the described IVE+LBT system and maximize the new exposure format in PD/A and additional mental disorders, it would be necessary to apply this technology to larger samples and future robust studies in an experimental design that includes a control group. Despite these limitations, however, we believe that this work contributes to the improvement of IVE of PD/A by innovative ICT tools by using LBT.

The IVE+LBT system described in the present research could represent a useful starting point and could prove essential in opening up new paths to extend its use to several additional disorders, especially those related to core locations involved both in the course of the disorder and the treatment. Examples of disorders that could benefit from this system are situational phobias, where the system can play a similar role enhancing the IVE tasks and gambling disorders where location-based technologies could be used to enhance stimulus control during the initial treatment of this non-substance-related disorder, reducing the abstinence violation. At the present time, our team is already working on the first pilot study using this technology for this last type of population [378]. We consider this line of research particularly relevant for future studies that wish to evaluate the role of these devices on treatment motivation, patient commitment during exposure tasks, and therapeutic adherence. Future studies could also address the inclusion of assessment tools in the system. That is, in order to advance in the Ecological Momentary Assessment (EMA), the inclusion of the anxiety and avoidance level, even ratings of the beliefs in catastrophic thoughts could be done through the same platform, creating an electronic record that could offer a full picture of the IVE task performed by the patient. The results of this study suggest that using the 
Chapter 5. Validation and experimentation of the platform in case studies with real patients.

application to continue adding intervention components during IVE exposure tasks can be very beneficial for the patient, following scheduled ways of administering psychological support as previous studies already have done [379] and reaching lower dropout rate, and better adherence to treatment.

The design of these novel strategies to target psychological disorders and enhance key therapeutic components could be benefited by the media-based tool development and would constitute very promising future research aims. In the case of smartphone devices, they also offer innovative advantages and it is imperative to continue exploring new possibilities. 
5.2. Enhancing therapeutic components in the treatment of gambling disorder 118 using location-based technologies

\subsection{Enhancing therapeutic components in the treat- ment of gambling disorder using location-based technologies: A case study of two participants' experiences.}

Gambling Disorder (GD) is a non-substance-related disorder leading to clinically significant impairment in different important areas of life, such as job, educational opportunities and significant relationships. GD represents an emergent public health problem with prevalence ranging up to $5 \%$. Cognitive Behavioral Therapy (CBT) is considered the treatment of choice, being stimulus control (SC) and exposure with response prevention (ERP) core components. In spite of the efficacy of CBT, SC and ERP are not easy components to deliver and they are hard for the patients. There are inherent difficulties, related to the commitment, for those people who suffer GD and high attrition rates and relapses are generally present. Thus, it becomes important to maximize key therapeutic components and its motivational features by some kind of support, as well as to explore new ways to enhance the patient's compliance in regard to SC and ERP components. The use of smartphones is a very useful option to initiate and sustain these key components. The main purpose of these case studies is to describe the use of location-based technologies in SC and ERP components during the treatment of two patients diagnosed with GD while assessing the acceptability and usability of the system by the patients. The Symptoms platform was used during the SC and ERP modules. The patients reported positive expectations, high satisfaction scores, and overall satisfactory experience. The work contributes to improving the disorder by providing tools during the treatment's crucial components and by offering a starting point that opens up new paths for psychological interventions through the use of smartphone devices which offers promising possibilities.

\subsubsection{Introduction}

Gambling Disorder (GD) is a non-substance-related disorder defined as a persistent and recurrent problematic behavior leading to clinically significant impairment or distress ([55]). Pathological gamblers (PG) usually need to bet with increasing amounts of money to accomplish the desired feeling of excitement and show an 
Chapter 5. Validation and experimentation of the platform in case studies with real patients.

irritable emotional state when they try to cut down or stop gambling. Although $P G$ repeatedly attempt to control, cut back or stop gambling, it is unsuccessful. Distressed feelings are common triggers of GBs and when PG lose money they will usually gamble again to "chase losses". PG are often worried about gambling (i.e., thinking about past experiences, planning next wagers or thinking about different ways to get money for betting) and lie about to what extent they are involved. For this reason, different important areas of life such as job, educational opportunities and significant relationships, can be affected. The complexity of the GD characteristics is associated with a high comorbidity with other psychological disorders ([380-382]) such as mood and anxiety disorders, substance use disorders, personality disorders (i.e., borderline or antisocial), attention-deficit hyperactivity disorder (ADHD), impulse control disorders, and schizophrenia ([380, 383-386]). GD represents an emergent public health problem. In Spain, a study carried out by the Directorate General for the Regulation of Gambling, DGOJ (2015) [387] shows that $24.3 \%$ are non-gamblers, $69.4 \%$ non-risk gamblers, $4.4 \%$ at-risk gamblers, $1 \%$ problem gamblers, and $0.9 \% \mathrm{PG}$ at any time in their life according to the NODS criteria.

The first-line treatment for GD is Cognitive-behavioral therapy (CBT) [388390] and it has been further reinforced by systematic review and meta-analysis efficacy studies that report finding important and long-lasting improvements [391]. The core features of CBT therapy for gambling disorder are stimulus control (SC), curtailing exposure to gambling opportunities and cues, and exposure with response prevention (ERP) which aims to prevent playing behaviors and confront the patient with overwhelming urge with the aim to habituating or extinguishing it [392, 393]. SC is introduced initially in order to avoid gambling cues and establish an abstinence period. After, ERP is incorporated for achieving the habituation process of the urge to gamble considering the presence of a particular emotional reaction or gambling related stimulus. Both components are well stablished [394]. In spite of the efficacy of CBT, SC and ERP, these components are not easy to deliver and they are hard for the patients. There are inherent difficulties, related to the commitment during SC, for those people who suffer GD and high attrition rates and relapses are generally present [395]. This leads to inherent treatment difficulties for people who suffer GD and therefore, it becomes important to enhance key therapeutic components and its motivational features.

Media-based tools have contributed to the development of new strategy designs 
5.2. Enhancing therapeutic components in the treatment of gambling disorder 120 using location-based technologies

to target psychological disorders. In recent years, there has been an increased interest in using technology-associated psychological interventions as a form of treatment for psychological disorders, including GD. Several studies have been conducted in order to improve the effectiveness of psychological treatments or clinical utility. The first promising technology was virtual reality (VR), which emerged as an effective tool for psychological disorders, reporting efficacy in the treatment of GD and the ERP component [396, 397]. Furthermore, media development has led to the use of the Internet to deliver CBT, obtaining very adequate results by RCT [398-401], systematic review or meta-analysis [391, 402] in which the efficacy of these self-guided treatments has been confirmed in many countries. The implementation of Information and Communication Technologies (ICT) for psychological intervention has been empirically tested. Along with the technology itself and the clinical effectiveness, the issue of acceptability of the technologyguided treatment is important when considering whether or not the use of ICT will affect the therapeutic adherence and outcomes [354]. Acceptability refers to the degree to which users are satisfied or at ease with the service and willing to use it [355] and influences whether a treatment is perceived as fair and reasonable, appropriate, and non-intrusive in addressing a problem [356, 357]. In the Technology Appraisal Guidance from the United Kingdom, it is considered a priority to properly assess intervention acceptability in treatments and the variables linked to it; expectations, satisfaction, and usability. This in turn, makes these variables become crucial features in psychotherapy results [358]. Several studies have focused on the expectations and satisfaction with different ICT tools such as VR or Internet-based treatment for delivering psychological treatments [10, $359,360]$ including gambling disorder [398, 400], reporting high satisfaction with the exposure component mediated by technologies. Studying acceptability and usability of an ICT has to take into account a very specific conceptualization inside the user experience, exploring the ease of use of a product by a specific user with clearly defined context and goals with the aim of enhancing the ICT development. Even though few studies have addressed this topic [357, 361, 362], the before mentioned ICTs have shown to be well-supported regarding acceptability issues.

The large increase in mobile phones and smartphones over the years (Taylor and Silver, 2019) offers additional and largely unexplored advantages to implementing psychological treatments in different mental disorders with the support of these technologies [363]. Smartphone portability could be very useful in a variety 
Chapter 5. Validation and experimentation of the platform in case studies with real patients.

of feared situations in which GD symptoms happen. Specifically, the locationbased technologies (LBT) with personalized feedback of the patient position could enhance key therapeutic components in specific disorders and could signify a starting point to initiate and sustain the behavior required during SC and ERP components. Consequently, it can lead to the improvement of the patient suffering pathological gambling by maximizing the motivation and commitment. Although LBT could become a tool when prescribing some therapeutic components, it will be upon the patient to carry out the tasks on his/her own in several situations where gambling happens, both in the offline gambling and online gambling. The most prevalent way of gambling is offline [387], but since online gambling was legalized in Spain in 2012, it has been considered the second cause of GD for patients undergoing treatment [403] and LBT can be very useful.

This study assumed a set of advantages of using these devices: a) the therapist can ensure that the patient actually is committed to the SC; b) the therapist can ensure that the patient stays in the exposure situation for the necessary time in order to fulfill the goals during the ERP component; and c) the patient's motivation and commitment can be enhanced and supported by the mobile device. In previous works, when carrying out the treatment, the context and LBT have been considered and treated as a variable. In the study of Addepally and Purkayastha (2017) [124], they looked at using a mobile application (app) that would monitor the location of depressed people. The app detected if the depressed patients were in lesscrowded areas (a frequent trait in depressed individuals according to the authors) and, if affirmative, the patient would receive therapeutic strategies and self-help assessment through notifications. In a case report study [364], a patient suffering from obsessive-compulsive disorder was allowed to configure alarms in case they remained for a long time without changing places using an app that tracked their position. In a case study carried out by our research team, LBT was used during the IVE component in the Unified Protocol treatment of a 47-year-old patient with Panic Disorder and Agoraphobia. The focus of the study was to enhance key therapeutic components during in vivo exposure and the patient reported positive expectations, high satisfaction scores, and overall satisfactory experience (5.1). However, to the best of our knowledge, there are no reports that account for LBT in a smartphone application for the treatment of GD during SC and ERP.

The main aim of this case study with two participants is to describe the use of LBT in SC and ERP components during the treatment of two patients diagnosed 
5.2. Enhancing therapeutic components in the treatment of gambling disorder 122 using location-based technologies

with GD while assessing the acceptability and usability of the system. The hypothesis for this study is that the location-based technology would enhance the therapeutic components during the treatment of the GD by a) enhancing motivation and patient commitment, and b) being well-accepted by the patient.

\subsubsection{Method}

\section{Patients}

Patient 1 is a 28-year-old male with higher education, who worked in a family company and was diagnosed with problematic gambling according to the Spanishlanguage version of the National Opinion Research Center DSM-IV Screen for Gambling Problems (NODS [404]). He had not previously received treatment for this problem. He lives with his parents and has been in a relationship with his girlfriend for over two years. He places bets on sports, especially soccer, and can't remember exactly when the betting started although he thinks that it probably started more than five years ago when he and his friends used to bet on soccer matches as a way of entertainment. During the last year before the treatment, he started to bet alone and spent the majority of his free time betting. The patient had no debts, but he recognized that he spent money in uncontrolled ways during his gambling sessions. His main thoughts are focused on his sports betting skills and "the possibility of winning a lot of money through gambling", in addition he described himself as a competitive person who likes to win. He didn't identify specific emotions linked to gambling but indicated feeling a kind of thrill while gambling and linked to the possibility of winning money. He started to have significant problems, especially severe arguments with his girlfriend because of the time he spent betting or thinking about it. It was then when he perceived gambling as a situation which was out of his control and decided to request psychological help. He also decided to talk to his parents by himself to inform them about the situation and received everyone's support. At the beginning of the treatment, no co-therapist was involved given that the patient was not currently gambling, had not acquired any debts and had reported his gambling situation to his significant people. His girlfriend's contact information (with her consent) was noted in case it was needed at any time during therapy. There was no substance abuse and the patient wasn't receiving pharmacologic treatment at the time. The patient mentioned having some social problems probably linked to the social anxiety that 
Chapter 5. Validation and experimentation of the platform in case studies with real patients. 123 can appear in those who are willing to receive psychological treatment once a gambling problem is addressed. When the patient attended therapy, he was trying to stop gambling.

Patient 2 is a 46-year-old single male with basic studies and who was employed at a company. He met the Diagnostic and Statistical criteria according to the Spanish-language version of the National Opinion Research Center DSM-IV Screen for Gambling Problems (NODS [404]). He had not previously received treatment for this problem. He mostly plays slot machines and additionally places bets on different types of gambling. He started to play in 2009 as a way of entertainment, coinciding with the acquisition of a credit for the construction of his new house. Soon he started to think about the possibility of gambling as a way of winning money to pay for some of his expenses. He started to play more during the day, between 10 minutes to 3 hours, changing the places where he played but with 3-4 favorite places. One day he spent more than 1,000 euros playing slot machines and he progressively ran up important debts of approximately 23,000 euros from credit banks, friends and his work company. The main thoughts stated by the patient were "Now I'm going to win and I will be able to recover the loss" or "today I am going to be lucky". Even when trying to stop the gambling behavior, he has never succeeded. The patient identified some negative emotions strongly linked to the gambling behavior such as feeling alone. His sister and brother know about the situation and his brother is the person involved in the therapy process as a co-therapist. There is no substance abuse and no other psychological diagnosis. The patient wasn't receiving pharmacologic treatment at the time. As the patient's positive characteristics and strengths it is important to note that he is very dynamic, with significant social support and a wide range of pleasure activities which he usually practices on a daily basis. When the patient attended therapy, he was trying once again to stop gambling after significant economic and family problems.

\section{Therapist}

The therapist was a member of our research team with more than ten years of experience in the therapeutic field including both technology-mediated and psychological intervention. 
5.2. Enhancing therapeutic components in the treatment of gambling disorder 124 using location-based technologies

\section{Measures}

Several common psychiatric testing measures were used in order to establish the diagnosis and to evaluate the effects of the intervention. However, given that the component under study was SC and ERP by location-based technologies $(\mathrm{SC}+\mathrm{LBT}$; ERP+LBT), only the measures related to this component will be presented in the paper (with the exception of the diagnosis measures).

Diagnosis measures and measures for the target behaviors:

\section{Primary Outcome Measures}

- NORC DSM-IV Screen for Gambling Problems (NODS [405], 1999 Spanish version by Becoña [404]). The NODS is a hierarchically structured 17-item screen that is designed to assess the at-risk, problem, and pathological gambling. It refers to the gambling experience both throughout the person's life and in the last year, with the alternatives being dichotomous (Yes / No). The total score ranges from 0 to 10 (1-2 affirmative items correspond with at-risk gambling; 3-4 items with problem gambling; and 6 or more with pathological gambling). The data obtained on specificity and sensitivity is good, its test-retest reliability is 0.98 , and its validity is excellent considering that it corresponds strictly to the DSM-IV criteria.

- Target behavior scales (adapted from Marks and Mathews, 1979 [366]) used to identify problem situations because of gambling. The target behaviors were defined as the behaviors linked to gambling and creating substantial impairment in the patient's daily life. The patients rated the level in terms of the overwhelming urge or craving state prior to engaging in the specific gambling behavior $(0=$ nothing; $10=$ maximum).

\section{Measures for expectations and satisfaction with the ICT system:}

- Expectation and satisfaction scale (adapted from Borkovec and Nau [367]) regarding the SC/ERP+LBT component used previously in our research group in other ICT contexts [359, 368], including pathological gambling [396]. This questionnaire was used to measure patient's expectations, before, and satisfaction, after, the SC/ERP + LBT component. The questionnaire includes six items; how logical the SC/ERP +LBT component seemed, to 
Chapter 5. Validation and experimentation of the platform in case studies with real patients.

what extent it could satisfy the patient, whether the patient would recommend this component treatment to others, whether it would be useful in treating other problems, the component's usefulness for the patient's problem, and to what extent it could be invasive. This last item was considered a key factor in assessing the ICT system during SC/ERP. Due to the main characteristics of the LBT, it was important to assess any disruption, annoyance or intrusion on the patient's privacy caused by the ICT. Both parts (expectations and satisfaction) ranged from 0 to 10 , being $0=$ "not at all" and $10=$ "very much".

Measures for acceptability and usability of the ICT system:

- System Usability Scale [370] is one of the most used tools for assessing perceived usability [371]. It consists of ten items, half of them are written in a direct style and the other half in an inverse style. A five-point scale is used for rating the level of agreement, from 1 (strongly disagree) to 5 (strongly agree). This scale has a score contribution of the scale position minus 1 for the items 1,3,5,7 and 9, and 5 minus the scale position for the items 2,4,6,8 and 10. A formula is used to calculate the score as a percentage scale from 0 to 100 .

\section{Treatment}

The patients received an intervention based on cognitive behavioral treatment (CBT) comprised from 8 sequential therapeutic modules: motivation for change, psychoeducation, stimulus control (e.g., self-prohibition and blocking of usual gambling) and responsible return of debts (in the case of patient 2), cognitive restructuring, emotion regulation, planning of significant activities, coping skills and exposure with response prevention, and relapse prevention. The sessions included in each module were delivered weekly in around 1-hour sessions. The $\mathrm{SC}+\mathrm{LBT}$ and ERP+LBT component was carried out during the stimulus control and exposure with response prevention modules, respectively, keeping the structure of the aforementioned CBT-based intervention and including the location-based technologies which allowed the patient to receive personalized messages during the treatment process and specifically during the SC and ERP components. That is, during the exposure with response prevention modules, the patients were exposed to their main target behavior and used the location-based technology 
5.2. Enhancing therapeutic components in the treatment of gambling disorder 126 using location-based technologies

included in the study. The program's content, including the eight modules with objectives and contents can be seen in Table 5.3. All of these modules exhibit a similar structure: a therapeutic content part presented with text, exercises and activities, a brief summary of the module, and tasks to complete before continuing through to the following modules. 
Chapter 5. Validation and experimentation of the platform in case studies with real patients.

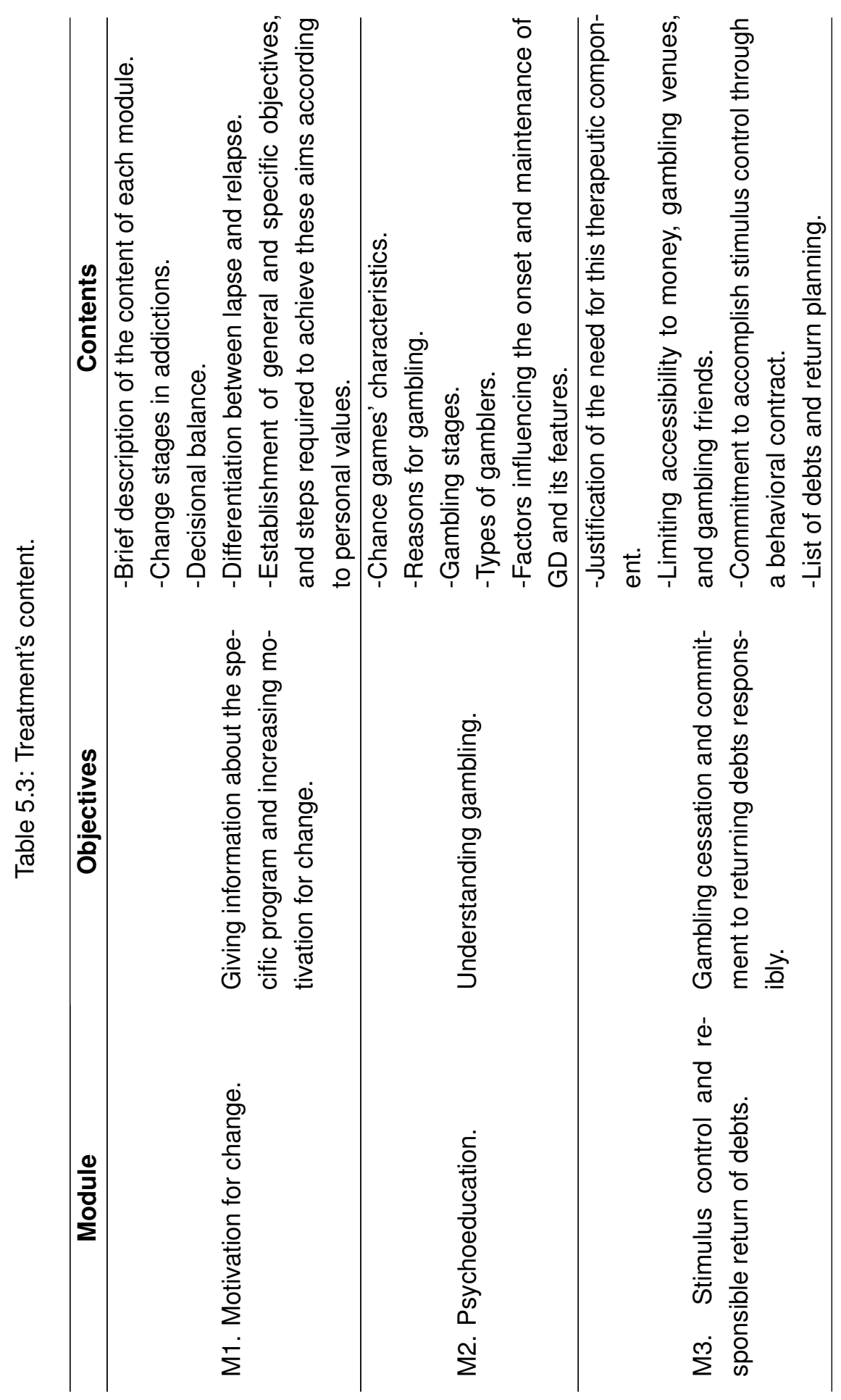




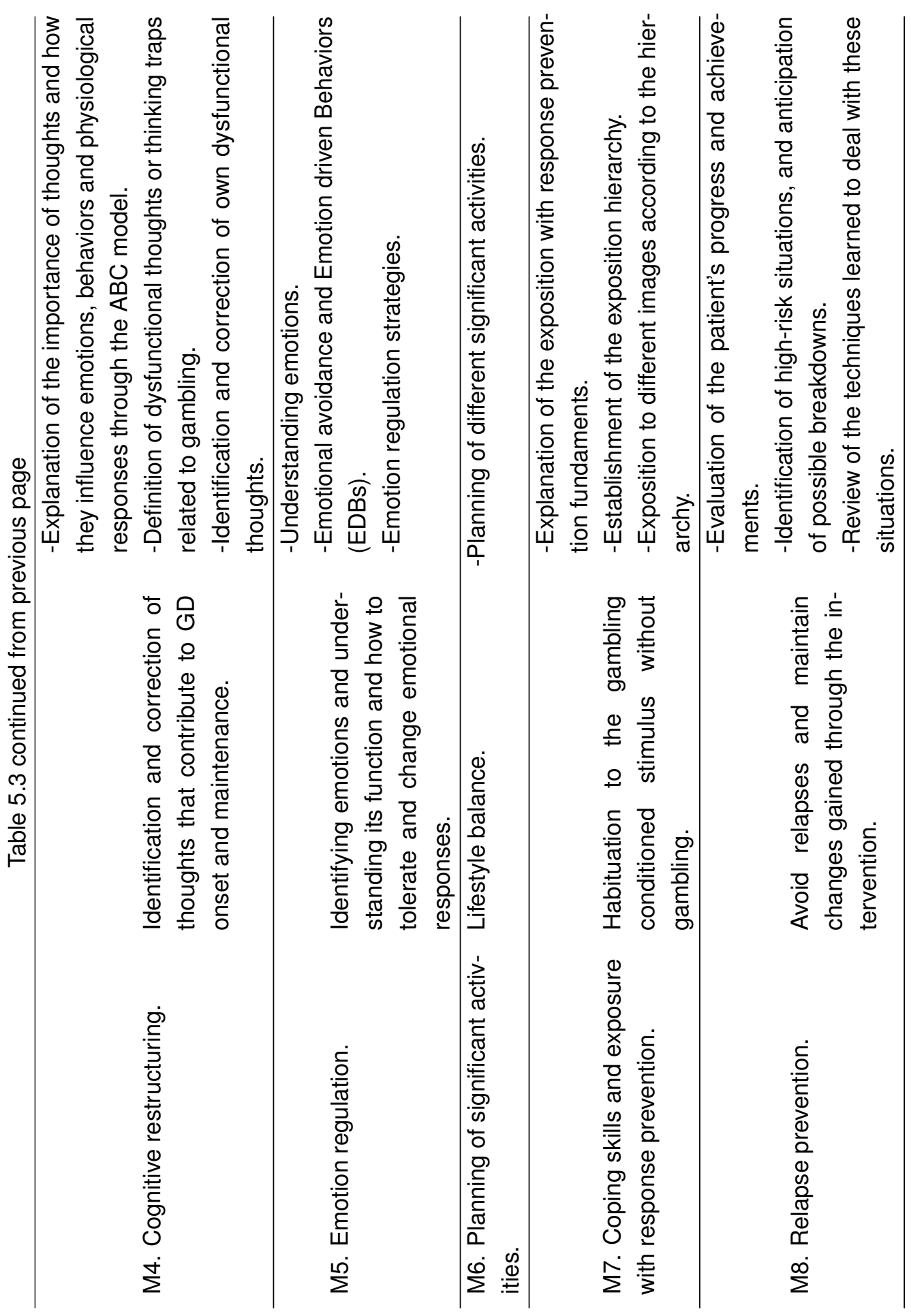


Chapter 5. Validation and experimentation of the platform in case studies with real patients.

\section{System Description}

For the present study the Symptoms platform was used, adapted to the gambling disorder pathology considered for this work. The full tool has been described in the section 5.1.2.

\section{Design and Procedure}

This study follows a case study with two participants. The patients asked for help at the Jaume I University Anxiety Disorders Clinic, Spain. First, they underwent a screening assessment and, having met the inclusion criteria, they then signed a consent form to participate in the study. Inclusion criteria included the following: a) meeting the gambling disorder diagnostic criteria (pathological or problematic gambling) and b) providing written, informed consent. Exclusion criteria included: a) suffering from a severe mental disorder (schizophrenia, bipolar disorder, and alcohol and/or substance dependence disorder); b) medical disease/condition that prevents the participant from carrying out the psychological treatment; or c) receiving another psychological treatment during the study. The assessment consisted of two 60-minute sessions to evaluate the diagnosis and establish the target behaviors related to the gambling behavior. The NODS was carried out in the first session and the second session was used to conduct other self-report measures and establish the patient's target behaviors. The rest of the measures linked to the treatment were carried out in the context of the full therapy. Given that the component under study was the SC/ERP+LBT, only the measures related to this component have been presented in the paper and emphasized in the measures section. Following the assessment and before starting the treatment, the therapist explained the basis of the treatment and the use of LBT to support the mentioned therapeutic components. The patients agreed to take part in the research. The expectation with the SC/ERP+LBT components was evaluated by the patients before the treatment was conducted.

Once the consent form for participating in the research and the consent form to use the LBT were filled out properly, the treatment started. The target behavior scale was assessed before each treatment session. Before starting the SC module, therapist and patient created the core situations linked to the gambling behavior and in consequence their target behaviors. The locations were positioned by the LBT and configured to receive the notification when the patient arrived at these 
5.2. Enhancing therapeutic components in the treatment of gambling disorder 130 using location-based technologies

core places during SC and ERP. The app was installed on their own smartphone. During the SC module, every time the patient arrived at one of the core places, they received a notification with the particular message configured previously by the therapist "You are in a risk zone, because it has been a place of gambling, remember that it is now important not to stay here". After the first use of the app, following the recommendations given by the theoretical framework of ICT usability $[370,371]$ the System Usability Scale was filled out by the patients. During the ERP module, every time the patient arrived at one of the core places during the exposure tasks, they received a notification with the particular message configured previously by the therapist "You are in a relevant place, the exposure begins, if there is an urge to gamble, use the strategies you have learned and leave the place when it has decreased". Following all the exposure tasks and after treatment, the satisfaction with the SC/ERP+LBT components was measured by the patients with the self-report measures, as well as the ICT component usability assessment.

\subsubsection{Results}

Patient 1 carried out the weekly treatment for a period of 4 months and patient 2 , who is still undergoing treatment, carried out 3 months of weekly treatment during the time of the study. Target behaviors

In Figure 5.7, the main target behaviors established by the patients and therapist regarding the gambling behavior, as well as the overwhelming urge ratings from the patients can be found. As shown in Figure 5.7 and 5.8, they are "sports betting" (for patient 1) in which the patient highlights the "excitement and urge to follow a strategy and the possibility of winning money" and "slot machines" (for patient 2) where the patient emphasizes a "strong overwhelming urge to gamble and distrust regarding his own capacity to resist it". The selected main target behaviors caused a certain (severe in the case of patient 2) level of impairment in different areas of the patients' life. Once the treatment started, there were no relapses reported by the patient or the co-therapist. Even though it was not a goal of the present study, preliminary results regarding the overwhelming urge in the target behavior show an important score reduction in the scheduled situations, supported by LBT during the treatment. 
Chapter 5. Validation and experimentation of the platform in case studies with real patients.

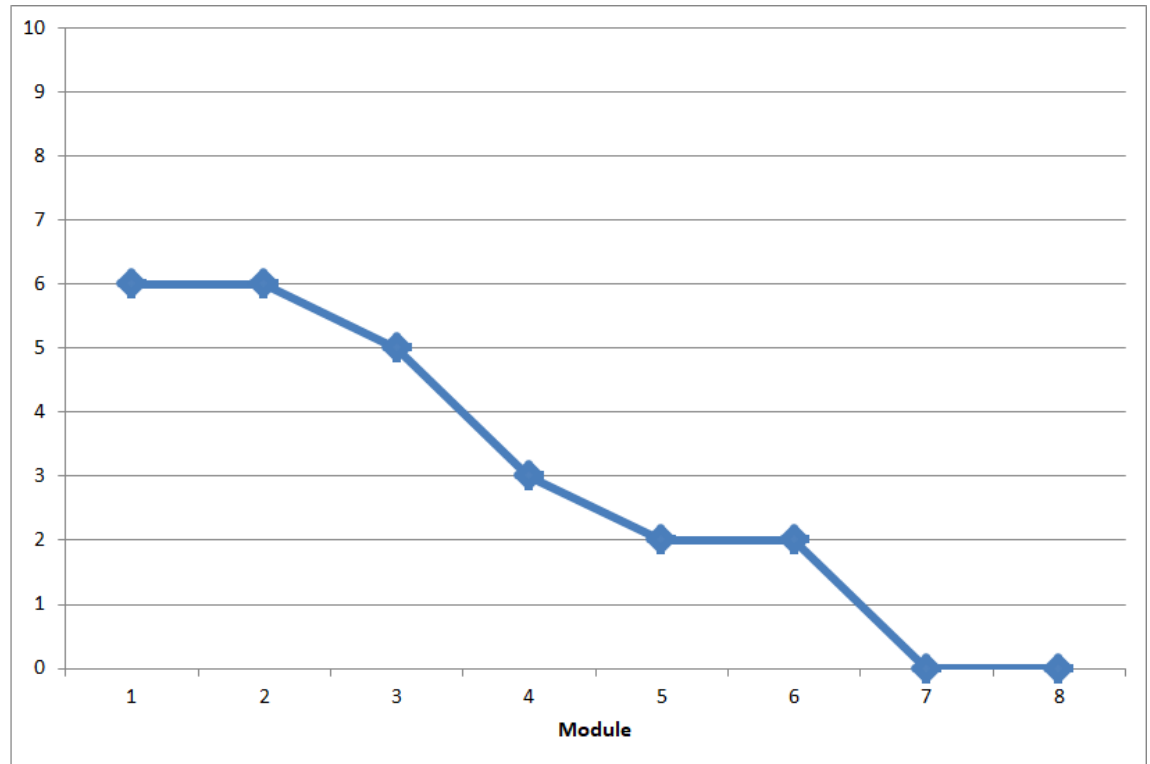

Figure 5.7: Overwhelming urge to play slot machines (0-10) and distrust regarding his own capacity to resist it for patient 2.

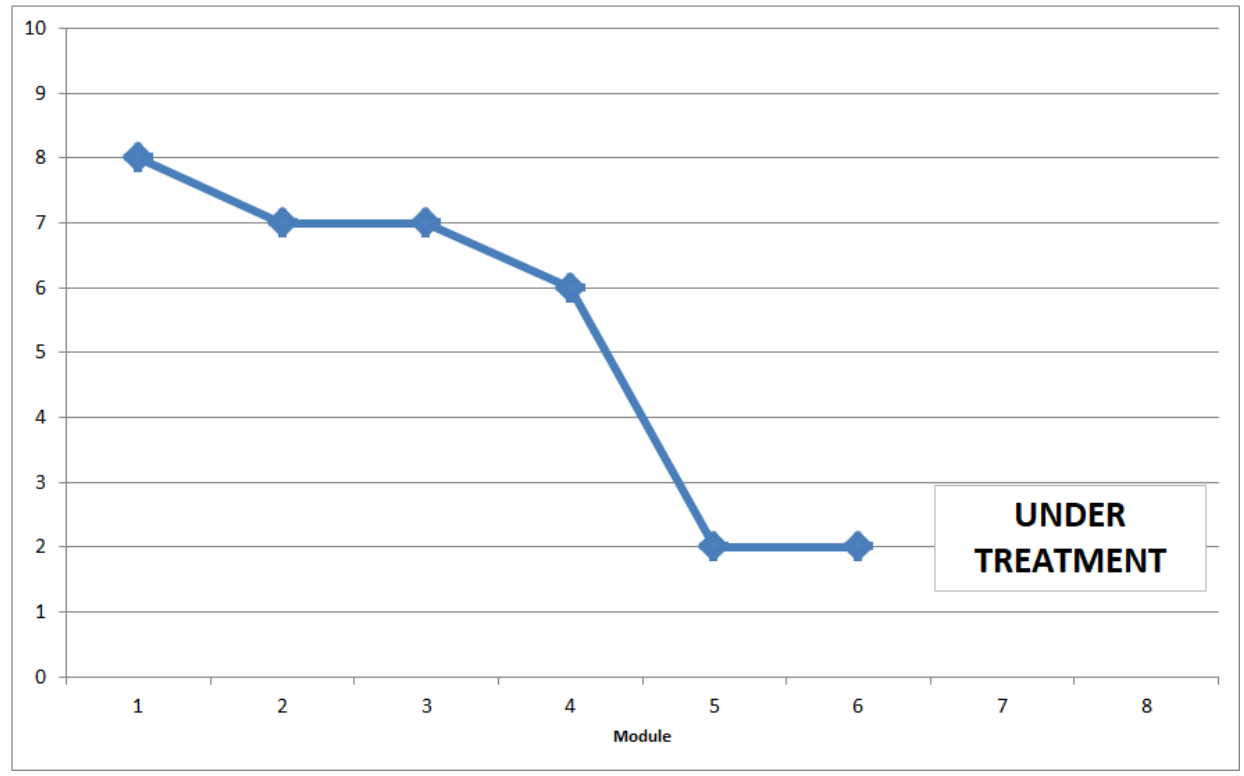

Figure 5.8: Appettive urge to playing slot machines (0-10) and distrust about his capacity to resist it for the patient 2 .

\section{Expectations and satisfaction regarding the SC/ERP+LBT components.}

\section{Patient 1}

He reported high expectations before starting the treatment and a high satisfaction after receiving it. Specifically, the patient considered that it could be invasive in a moderate form before starting the treatment, but after the intervention, invasiveness was assessed as low (Figure 5.9). 


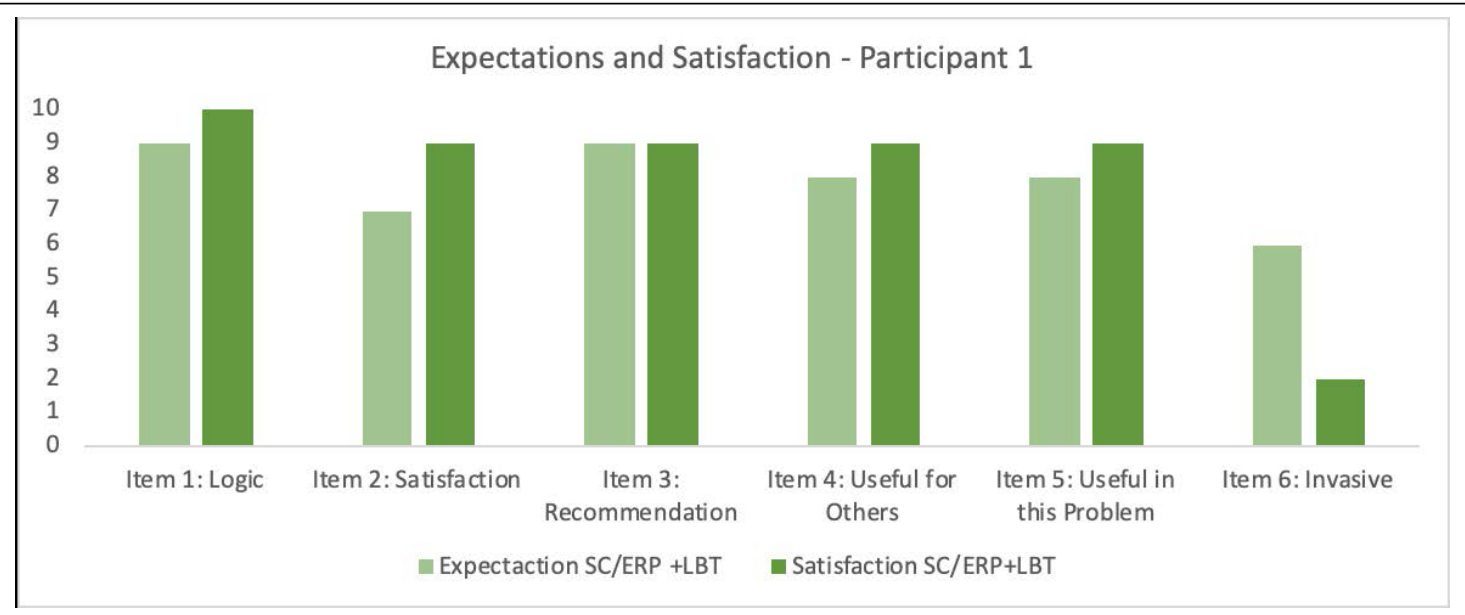

Figure 5.9: Expectation and satisfaction with the SC/ERP+LBT by patient 1

The perceived usability score is high in both assessment moments (first app's use and after treatment) and it increases slightly after the intervention (Table 5.4). In particular, one of the important aspects that improves is the confidence when using the system.

Table 5.4: SC/ERP+LBT usability test for patient 1.

\begin{tabular}{lll}
\hline \multicolumn{1}{c}{ Items } & First Use & After Intervention \\
\hline 1: I think that I would like to use this system frequently & 4 & 4 \\
2: I found the system unnecessarily complex & 1 & 1 \\
3: I thought the system was easy to use & 5 & 5 \\
4: I think that I would need the support of a technical & 1 & 1 \\
person to be able to use this system & \\
5: I found that the various functions in this system were & 5 & 4 \\
well integrated & \\
6: I thought that there was too much inconsistency in this & 2 & 1 \\
system & \\
7: I would imagine that most people would learn to use & 5 & 5 \\
this system very quickly \\
8: I found the system very cumbersome to use \\
$\begin{array}{l}\text { 9: I felt very confident using the system } \\
\text { 10: I needed to learn a lot of things before I could get } \\
\text { going with this system }\end{array}$ \\
\hline
\end{tabular}

The overall value for satisfaction and usability was 87.5 points after the first use and 92.5 after treatment, which according to the qualitative scale developed 
Chapter 5. Validation and experimentation of the platform in case studies with real patients.

by Bangor, Kortum, and Miller (2008) [373] means that the system is within an acceptable range, with adjectives rating between "excellent" and "best imaginable".

\section{Patient 2}

Currently, this patient is undergoing the intervention, so we have only reported data about expectations at pre-intervention (Figure 5.10) and the system usability after the first use of the system (Table 5.5). He reported high expectations before starting the treatment and a high perceived usability after the first use, during the SC component. He did not consider it at all invasive.

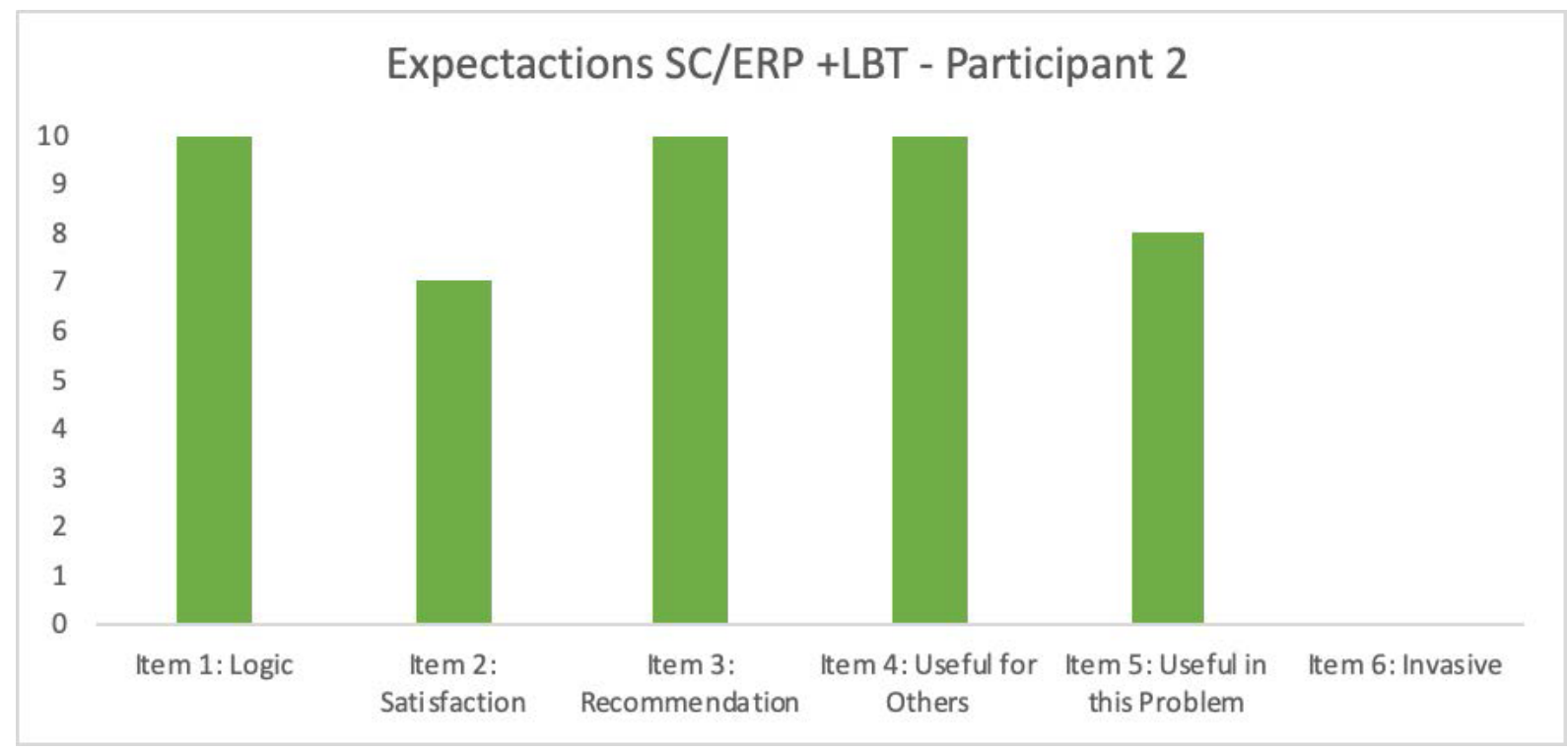

Figure 5.10: Expectation with the SC/ERP+LBT by patient 2

The overall value for satisfaction and usability was 100 points for patient 2 (after the first use). This score is the maximum of the scale and in the same way as patient 1 and according to the qualitative scale developed by Bangor et al. (2008) [373], it means that the system usability perceived for this participant was the "best imaginable".

Finally, we obtained some qualitative opinions from the patients regarding their experience. Here are some of the opinions expressed by the users after the SC/ERP+LBT:

Qualitative opinion (Patient 1):

- Although I thought it would invade my privacy, knowing that it was used for this specific purpose was not a problem. 
5.2. Enhancing therapeutic components in the treatment of gambling disorder 134 using location-based technologies

Table 5.5: SC/ERP+LBT usability test for patient 2.

\begin{tabular}{ll}
\hline \multicolumn{1}{c}{ Items } & First Use \\
\hline 1: I think that I would like to use this system frequently & 5 \\
2: I found the system unnecessarily complex & 1 \\
3: I thought the system was easy to use & 5 \\
4: I think that I would need the support of a technical & 1 \\
person to be able to use this system & \\
5: I found that the various functions in this system were & 5 \\
well integrated & \\
6: I thought that there was too much inconsistency in this & 1 \\
system & \\
7: I would imagine that most people would learn to use & 5 \\
this system very quickly & 1 \\
8: I found the system very cumbersome to use \\
9: I felt very confident using the system \\
10: I needed to learn a lot of things before I could get \\
going with this system
\end{tabular}

- It did not use a lot of battery and I haven't had any technical problems.

Qualitative opinion (Patient 2):

- The system reinforces the aspect of avoiding going to prohibited sites.

- It is a reinforcement and one has in mind that he has made a mistake.

- I haven't found the system invasive.

\subsubsection{Discussion}

The results obtained in this case study including two participants' experiences show that the use of LBT presents relevant implications for the innovation in the treatment of gambling disorder with different types of severity. The positive consequences of monitoring the patients during the treatment seem to be several; 
Chapter 5. Validation and experimentation of the platform in case studies with real patients.

1) the certainty that the patient's efforts are being recorded and visualized by the therapist, 2) the technology is well-accepted by the patient, showing positive expectations and high satisfaction, and 3 ) it has the possibility of increasing the commitment to the scheduled SC tasks (before) and ERP components (after). The reported positive expectations and high satisfaction scores by the patients coincide with previous studies showing that ICT treatments are well-accepted [10, $359,360]$, also in pathological gambling studies [398, 400]. Specifically, LBT has been well-assessed in a previous pilot study with another pathology (see section 5.1) obtaining promising preliminary results. These results with more advanced technology reach a high importance, since a positive relation between expectations and satisfaction with the ICT treatments and intervention efficacy have been found in [374]. Consequently, it is important to continue improving the treatment by innovative tools which can have direct implications on effectiveness.

An important aspect is to what extent the LBT considered for the study could be invasive. Patient 1 considered invasiveness as low especially after the intervention and patient 2 expected that the system would not be invasive at all. Due to the type of ICT considered in this work and the aimed disorder, the data on intrusiveness are especially relevant. The loss of privacy due to detailed information about the system usage being sent to the therapist could have created interference with the opinion about the system. However, this was not the case in this study and LBT was not considered invasive or an interference in the fulfillment of the SC and ERP components. Finally, usability results reported the patient's satisfactory experience with the system. According to the qualitative scale developed by Bangor, Kortum, and Miller (2008) [373] this means that the system is within an acceptable range, with adjectives rating between "excellent" and "best imaginable". Based on the technology acceptance model, these authors have suggested that one of the factors that can be related to the intention to use a product in the future is ease of use $[183,188]$. Therefore, the usability, which is already an important attribute in the use of any technology [375], becomes a prerequisite in the use of technology for mental health. Technology must be completely transparent from the beginning, otherwise, a slow learning curve or high frustration during use would interfere with the therapy and negatively influence the outcome of the treatment. Therefore, efforts to research and ensure the usability of new ICT approaches are crucial.

Previous research has focused on the importance of using personalized feedback interventions for gambling disorders [406] and how it can show success 
5.2. Enhancing therapeutic components in the treatment of gambling disorder 136 using location-based technologies

as a low-cost intervention for reducing problematic behavior in addictions. The use of innovative ICTs such as LBT can be a first step to continue working on the treatment of this pathology. Specifically, the development of particular ICT strategies as additional tools to guide the SC and ERP components can be useful in increasing overall functioning, enhancing the motivation and commitment of the patient with critical components of the therapy and reducing the abstinence violation, relapses and dropouts, especially important and high in pathological gambling ([407]).

From a technical point of view and given the simplicity of the creation of the application, the adaptability of this tool is also presented as a positive feature. Changing the indicated places or the information delivered depending on the therapeutic component (SC or ERP, for example) can be done fast and without requiring specific technical knowledge. This makes it easy to customize applications for different patients and therapeutic components. As was mentioned previously (Chapter 5.1), generating this type of in-situ intervention has always been somewhat complex, requiring time-consuming traditional methods or trusting the patient to carry out the indicated tasks. Thanks to this tool, this process has been streamlined, allowing the therapist to focus their attention on the therapeutic content and delegating the monitoring and delivery of the materials to the mobile device. Future improvements of the tool have been discussed in the previous section (Chapter 5.1) and could have an important utility in the context of pathological gambling. This is the case of including additional variables to improve the quality of the intervention, such as different messages arriving depending on the time (a message at the beginning of the ERP component, a message in the middle, and one at the end of this therapeutic component) or more complex content such as multimedia resources (images or videos) which can help during the exposure to the relevant target places for the gambling behavior. To extend the application of the tool beyond the intervention and include feedback in the form of questionnaires that are able to identify, for example, the overwhelming urge of the patient could also be very useful.

This study has some shortcomings. The main one is that it is a case study with the purpose of testing an innovative tool in the field of pathological gambling. In order to increase the confidence in the described SC/ERP+LBT system, it would be necessary to apply this technology to larger samples and future robust studies in an experimental design that includes a control group. In addition, the application 
Chapter 5. Validation and experimentation of the platform in case studies with real patients.

at the moment only allows for a basic configuration (information in text format and places) as was mentioned before. However, despite these limitations, we believe that this work contributes to the improvement of the disorder, providing tools during key components of the treatment by offering a starting point that opens up new paths for psychological interventions through the use of smartphone devices which offers promising possibilities. 

All we have to decide is what to do with the time that is given us.

Gandalf the grey.

This chapter summarizes the main contributions and findings of the study and discuss them in a broader context. In particular, in the fist section, we summarise the main research contributions and reflect on how they have addressed the initial research questions raised on Chapter 1 . Next, we discuss the limitations of the research work. Finally, we conclude the manuscript by suggesting a future research agenda.

\subsection{Main research contributions}

This thesis has covered all the stages in a software engineering project in particular, and a scientific research project in general. The thesis presented the analysis and elicitation of requirements, design and development of a platform capable of creating context-aware mobile applications for monitoring and intervening in mental health treatments, and the validation of the platform in real experiments.

The work begins by analyzing the current state of the art (Chapter 2) to address $\mathrm{O} / \mathrm{RQ} 1$ - what is the current state of the art in the use of mobile applications for psychological interventions?. For doing this a systematic review was carried out that identified the most addressed disorders and the technical features that have been used in this type of applications. The results indicated that the use of sensor and mobile technology is growing rapidly. In addition, the review results showed that depression and anxiety were the most popular disorders in terms of the number of studies and applications that focus on them. Regarding the 
technical characteristics, it is striking that many of the surveyed works were limited to transferring the traditional way of doing things, without taking advantage of many of the techniques offered by recent technological advances, such as artificial intelligence or geolocation. This review gave a general perspective that guided the direction that our platform development should follow.

To answer the O/RQ2 - what are the design considerations that must be taken into account so that a technological development applied to psychological interventions maximizes the chances of success?, we started with the results of the previous review to identify which areas and dimensions should be taken into consideration for the design and development of context-aware, mobile apps (Chapter 3). The methodology employed was the combined use of diverse qualitative methodologies to identify the key dimensions and, most importantly, to reflect on the relationships between these dimensions and their impact in the design of this type of apps for interventions. Not only does the resulting model take into account technological dimensions, but it also accounts for the dimensions relative to the environment in which the applications are used, and the dimensions pertinent to the mental disorders. In summary, the Technology area was based on Interface and Gamification dimensions; the Context contained Place, Spatial Relationship and Sense of Place dimensions, and Mental Health involved Profile, Diagnosis and Actors dimensions.

We took the two previous contributions as inputs for the design and develop of the Symptoms platform (Chapter 4), as the main engineering contribution that satisfactorily addresses O/RQ3 - can we develop a technological platform that allows therapists to create their custom mobile applications for psychological interventions in a simple way and without the need for technological knowledge?. The platform allows therapists to configure and create their own mobile applications for the intervention in hand, requiring only basic, user-level technological knowledge to interact with the platform. This permits, on the one hand, to increase the range and number of tools available to therapists who do not have technical knowledge but who can still create their own mobile apps adapted to the needs of their interventions. On the other hand, the platform ensures that the generated mobile app takes into account all the design considerations seen in Chapter 3 and lessons learned from the previous contributions (review - O/RQ1) and preliminary scientific results ([235, 408]). In chapter 4, we described the conceptual and technical architecture of the platform, and the technical development of the contained elements, accompanied 
by an use case on depression to ease the reader's understanding of the platform's features.

The platform is composed of three main elements: A Builder, a web application to allow therapists both to register their patients and intervention applications, and to configure and create mobile applications with the characteristics needed; one (or several) mobile applications, which are delivered to patients and are responsible for carrying out interventions (or evaluations) on the patient's mobile phone; and finally a Viewer, another web application which permits therapists to monitor and visualize relevant information from the patient collected during the psychological intervention.

The platform and accompanying tools and apps have been validated through in-lab and real-life experimentation to address the O/RQ4 - does the technological platform support psychological treatments for distinct mental disorders effectively? In-lab validation were traditional tests (e.g. test cases, dry run test, etc.) conducted under controlled conditions prior to use the tools and apps with real users on the street. The most important were real-life experiments with patients. In particular, we based our assessment on three real use cases: a patient suffering from panic and agoraphobia and two others suffering from pathological gambling (Chapter 5). The results of the experiments showed positive expectations and high levels of satisfaction, highlighting how positive this type of applications can be when they are included in treatments.

As stated in the introduction, each of the central chapters was devoted to answer one of the research questions. We strongly believe that the contributions resulting from this thesis get us closer to the general objective of the research work: Extending the number of technological tools available for therapists and mental health professionals to cope effectively with interventions related to mental health disorders. Allowing the clinicians themselves to create their own tools has a strong impact on the final interventions, according at least to the following points:

1. Quantity: Being able to easily design and create an application should promote the use, experimentation and validation of applications. We expect that these types of interventions will proliferate as therapists may share their experience with peers and colleagues.

2. Precision: As the applications are fully customizable, adapted to each patient, these can be used anywhere without the need for specific technical 
adaptations, and subject only to the needs of each patient, urban context and the criteria of each therapist. These adjustments are done via configuration, which means the two identical mobile apps can behave slightly different depending on the city were they are deployed. Consequently, this will allow the intervention to be much more precise and concrete, thereby improving adherence and results.

3. Power: Despite there are more and more applications available as demonstrated in the systematic review (Chapter 2, [291]), their technological characteristics are still under-explored. We hope that the Symptoms platform will allow mobile applications take greater advantage of the sensors and technologies that are available on smartphones and mobile devices. In addition to being more personal, more powerful, more reactive to the environment, this will make the resulting applications more context-aware and more efficient.

Technological developments and psychological treatments have always been considered differentiated sciences and possibly they are, however, there is a common part with enough space to specialize entire teams in it. Engineers often find it difficult to know the specific needs of clinicians and, vice versa, also for clinicians to know the available technological possibilities. This research is expected to shed some light in this direction to encourage more people to join that common space. Multidisciplinary teams with researchers from both fields will better understand what is needed in the therapy to relate it to the options offered by the technology, while offering the possibility to carry out these developments easily and quickly, positively impacting the society.

\subsection{Limitations}

This thesis is not free from limitations. It is a work that has gone a long way, from the identification of the problem and requirements, to the creation of recommendations or considerations and the development of the platform, to testing and validation of the tools in real case studies. In this journey, this thesis has navigated between two differentiated disciplines, mental health and computer science, and, consequently, this has generated different types of conflicts and limitations.

One of the most important limitations has been time. The time available to carry out all the research activities is simply limited: meetings with experts, software 
development and testing, and, above all, experimentation with patients. All these tasks required enormous amounts of time. Looking at the potential results of this thesis, it would have been desirable to deepen more in some aspects, for example, to have more meetings with psychologists, to extend the platform with more functionalities like profiling, and to pay more attention to other aspects all the platform such as usability and accessibility. Properly covering these aspects would surely have resulted in better tools for therapists than those reported in this thesis. On the positive side, though, the basis for achieving this objective is made and demonstrated in the findings of this thesis; It is a matter of investing time to go further and expand the results reported here.

If experimentation with patients is a time-consuming activity and is not completely under the control of the author of this thesis (also depending on psychologists, patients, etc.), the type of experiments described in Chapter 5 based on case studies represent the minimum standards from the perspective of Psychology. It would have been highly desirable to recruit a greater number of patients, idyllically reaching a complete RCT. Obviously, an RCT provides the highest standards in terms of efficiency, feasibility and assessment of the tools used in the intervention, but also would have required much more time, exceeding by far the typical temporal window in the doctoral program. The plan, recruitment and availability of patients depend on many factors which are often beyond the control of a single person and, therefore, are not easy to carry out. Despite this, some actions have been initiated to address these shortcomings as pointed out in the next section (Future Work).

Logistic-wise, another limitation is the current regulations of the doctoral program in computer science. The present manuscript was initially conceived as a compendium of scientific articles that each one described, independently, a phase of the investigation process. Unfortunately, the necessary requirements of a thesis to be considered "compendium of articles" were modified in the last year, including that at least three of the publications had an impact index at the time of writing the thesis manuscript. As is often the case in science, the times of scientific publication are very variable and unpredictable. The structure of this thesis is (unofficially) a compendium of scientific articles; however, given that all but Chapter 3 are still in the reviewing process and the definitive responses from the journals are still pending, the thesis is therefore not officially filed as "compendium of articles". Despite this administrative misunderstanding, the author wishes the reading of the work captures the reader's interest through a fluid and coherent thread, expecting 
that the submitted publications will be published definitively in the coming months.

From a technological point of view, the platform utilizes the latest technologies available in web development at that time, and hence we cannot state that the platform is powered by cutting edge technology. Basically for two reasons. First of all, technology changes fast, very fast; the speed at which the technology advances is always greater than that of development. For example, the initial development of the platform began with Angular version 2 and ended with version 8. A side-effect is that periodic software upgrades are not always simple and often involve more problems than improvements; although the platform is constantly updated, the one presented in this thesis is not the latest version. Secondly, public releases of the platform must rely on stable and consolidated technologies since it has been applied to real use cases and experimenting with unstable tools might have unintended consequences on the treatment and even on patient's health. Since patient's well-being is always a priority, making a patient dependent on technology during experimentation was never an option in the realm of this thesis.

Like all research work, if this thesis were to start again, there could possibly be things that would be done differently. We would try to go further and try to make the limitations affect as little as possible the research work. Perhaps that learning is part of the contributions that a doctoral thesis makes in a field, the methodology carried out itself, the mistakes made and the things that have been learned in that process.

\subsection{Future Work}

This work has provided the foundation on which further possibilities may arise. Making the creation of applications accessible to the therapists themselves, without the need for intermediate technical teams, and to take advantage of the relevance of the context and techniques to improve adherence in interventions, have been raw contributions that are expected to propel both advance developments and open many more research opportunities in the field.

From an objective point of view, as mentioned in the scientific productivity generated by the thesis, some awards have already been obtained. The impact of the achieved findings in this thesis is not only measured in terms of scientific publications but also as funded research projects and granted predoctoral scholarships. Of these scholarships, it should be noted that they pursue differentiated research 
objectives that are expected to lead to considerable growth of the platform in the future. In particular, research work is already being done on more complex analyzes taking advantage of contextual information. For example, the use artificial intelligence to make clinical predictions based on the trajectories or the behavior of the patients. Another line of research is the ability to predict if a person's behavior pattern is a symptom of another possible problem or becomes a proof that the treatment actually works. Another interesting aspect beyond the present thesis is to focus on intervention, for example, to directly communicate intervention-related information to the therapist or to the people involved in the treatment, and to deliver an immediate response at the right time and place to patients to alleviate their problem.

There is also room for improvement from a more technical point of view. To mane a few, new explorations can range from improving the technical characteristics of the platform and applying last technologies, to obtaining a better battery performance in the mobile applications, to applying heuristics methods to speed up analyzes that are performed on the server, to integrate external services such as weather services and social networks into the platform to gather more and better contextual information.

If we look at the adherence, it is already being investigated how to add new gamification techniques to the platform to reduce abandonment rates. These techniques can be based on virtual environments that make the patient much more aware of their status and the consequences through the interaction with a gamified or social application.

From a clinical point of view we have seen examples of contextual information in three dimensions. Firstly, the situation of depression, where behavior was analyzed and guidelines were provided. Secondly, the addition of places to visit, for disorder like agoraphobia, where the patient is encouraged to go to a specific place to expose himself to his fears. Finally, the incorporation of prohibited places where the patient is encourage to keep away from these places. However, the possibilities are enormous. For example, future lines may imply the analysis of trajectories to compare them with patients' moods, or the exploration of what characteristics of the social and built environment can influence most in the patient's mood and emotional perception so as to suggest those places that are more recommended to make an intervention a success.

These are only some of the research lines that may arise from this thesis, 
but it is expected that the on-going investigations aforementioned will open new possibilities as well as research challenges. Finally, the author of this thesis would like to emphasize that this work consolidates a line of research in the immediate future so that researchers, patients and society in general can benefit from an improvement in the quantity and quality of psychological treatments. In other words, the author would like that the work that has been done in the forge to help warriors have better weapons can overcome the evil that lies ahead. 


\section{Bibliography}

1. World Health Organization. Data and resources http ://www . euro. who . int / en / health - topics / noncommunicable - diseases / mental - health / data-and-resources/. [Online; accessed 06-March-2019]. 2019.

2. Trautmann, S., Rehm, J. \& Wittchen, H.-U. The economic costs of mental disorders: Do our societies react appropriately to the burden of mental disorders? EMBO reports, e201642951 (2016).

3. Vos, T. et al. Global, regional, and national incidence, prevalence, and years lived with disability for 310 diseases and injuries, 1990-2015: a systematic analysis for the Global Burden of Disease Study 2015. The Lancet 388, 1545-1602 (2016).

4. Evans-Lacko, S. et al. Evaluating the economic impact of screening and treatment for depression in the workplace. European Neuropsychopharmacology 26, 1004-1013 (2016).

5. World Health Organization. Mental Health Atlas. tech. rep. (2017), 32. https : / / apps . who . int / iris / bitstream / handle / 10665 / 272735 / 9789241514019-eng . pdf?ua=1.

6. González, S. G. et al. Psychological Treatments Features and Outcomes in Spanish Public Mental Health Centres. International journal of psychology and psychological therapy 14, 17-32 (2014).

7. Meyerbröker, K. \& Emmelkamp, P. M. Virtual reality exposure therapy in anxiety disorders: a systematic review of process-and-outcome studies. Depression and Anxiety 27, 933-944 (2010).

8. Opriş, D. et al. Virtual reality exposure therapy in anxiety disorders: a quantitative meta-analysis. Depression and Anxiety 29, 85-93 (2012). 
9. Powers, M. B. \& Emmelkamp, P. M. G. Virtual reality exposure therapy for anxiety disorders: A meta-analysis. Journal of Anxiety Disorders 22, 561-569 (2008).

10. Botella, C. et al. Virtual reality exposure in the treatment of panic disorder and agoraphobia: A controlled study. Clinical Psychology \& Psychotherapy 14, 164-175 (2007).

11. Choi, Y. H. et al. Effects of group experiential cognitive therapy for the treatment of panic disorder with agoraphobia. Cyberpsychology, Behavior, and Social Networking 8, 387-393 (2005).

12. Ma, P.-A. et al. Virtual reality interoceptive exposure for the treatment of panic disorder and agoraphobia. Studies in health technology and informatics 154, 77 (2010).

13. Peñate, W., Pitti, C. T., Bethencourt, J. M., de la Fuente, J. \& Gracia, R. The effects of a treatment based on the use of virtual reality exposure and cognitive-behavioral therapy applied to patients with agoraphobia. International Journal of Clinical and Health Psychology 8, 5-22 (2008).

14. Carlbring, P., Westling, B. E., Ljungstrand, P., Ekselius, L. \& Andersson, G. Treatment of panic disorder via the internet: $A$ randomized trial of a self-help program *. Behavior Therapy 32, 751-764 (2001).

15. Reger, M. A. \& Gahm, G. A. A meta-analysis of the effects of internetand computer-based cognitive-behavioral treatments for anxiety. Journal of Clinical Psychology 65, 53-75 (2009).

16. Peñate, W. \& Fumero, A. A meta-review of Internet computer-based psychological treatments for anxiety disorders. Journal of Telemedicine and Telecare 22, 3-11 (2016).

17. Kumar, Y, S., A, B., S, K. \& H, R. I. The Effectiveness of Internet-Based Cognitive Behavioral Therapy in Treatment of Psychiatric Disorders. Cureus 9 (2017).

18. Taylor, K. \& Silver, L. Smartphone ownership is growing rapidly around the world, but not always equally (2019). 
19. Statista. Number of smartphone users worldwide from 2014 to 2020 (in billions) https : / / ww . statista . com/statistics / 330695/ number - of smartphone-users-worldwide/. [Online; accessed 07-December-2018]. 2018.

20. Center, P. R. Smartphone Ownership Is Growing Rapidly Around the World, but Not Always Equally. https://pewrsr.ch/2MKmmns. [Online; accessed 07-December-2018]. 2019.

21. Luxton, D. D., McCann, R. A., Bush, N. E., Mishkind, M. C. \& Reger, G. M. mHealth for mental health: Integrating smartphone technology in behavioral healthcare. Professional Psychology: Research and Practice 42, 505-512 (2011).

22. Bert, F., Giacometti, M., Gualano, M. R. \& Siliquini, R. Smartphones and Health Promotion: A Review of the Evidence. Journal of Medical Systems 38, 1-11 (2014).

23. Cunningham, P. J. Beyond Parity: Primary Care Physicians' Perspectives On Access To Mental Health Care. Health Affairs 28 (2009).

24. Huxley, C. J., Atherton, H., Watkins, J. A. \& Griffiths, F. Digital communication between clinician and patient and the impact on marginalised groups: a realist review in general practice. British Journal of General Practice 65 (2015).

25. Rathbone, A. L. \& Prescott, J. The Use of Mobile Apps and SMS Messaging as Physical and Mental Health Interventions: Systematic Review. Journal of Medical Internet Research 19 (2017).

26. Pepin, R., Segal, D. L. \& Coolidge, F. L. Intrinsic and extrinsic barriers to mental health care among community-dwelling younger and older adults. Aging \& Mental Health 13, 769-777 (2009).

27. Rowan, K., McAlpine, D. D. \& Blewett, L. A. Access And Cost Barriers To Mental Health Care, By Insurance Status, 1999-2010. Health Affairs 32, 1723-1730 (2013).

28. Twomey, C., O'Reilly, G. \& Meyer, B. Effectiveness of an individually-tailored computerised CBT programme (Deprexis) for depression: A meta-analysis. Psychiatry Research-neuroimaging 256, 371-377 (2017). 
29. Ma, J., Li, C., Kwan, M.-P. \& Chai, Y. A Multilevel Analysis of Perceived Noise Pollution, Geographic Contexts and Mental Health in Beijing. International Journal of Environmental Research and Public Health 15, 1479 (July 2018).

30. Wang, R., Xue, D., Liu, Y., Liu, P. \& Chen, H. The Relationship between Air Pollution and Depression in China: Is Neighbourhood Social Capital Protective? International Journal of Environmental Research and Public Health 15, 1160. ISSN: 1660-4601 (June 2018).

31. Sallis, J. F. et al. Physical activity in relation to urban environments in 14 cities worldwide: a cross-sectional study. The Lancet 387, 2207-2217 (2016).

32. Saelens, B. E., Sallis, J. F. \& Frank, L. D. Environmental correlates of walking and cycling: findings from the transportation, urban design, and planning literatures. Annals of behavioral medicine 25, 80-91 (2003).

33. Ewing, R. \& Cervero, R. Travel and the built environment: a meta-analysis. Journal of the American Planning Association 76, 265-294 (2010).

34. Melis, G., Gelormino, E., Marra, G., Ferracin, E. \& Costa, G. The Effects of the Urban Built Environment on Mental Health: A Cohort Study in a Large Northern Italian City. International Journal of Environmental Research and Public Health 12, 14898-14915. ISSN: 1660-4601 (Nov. 2015).

35. Statista. Mobile App Usage - Statistics and Facts. https://www . statista. com/topics/1002/mobile-app-usage/. [Online; accessed 07-December2018]. 2017.

36. Mosa, A. S. M., Yoo, I. \& Sheets, L. A Systematic Review of Healthcare Applications for Smartphones. BMC Medical Informatics and Decision Making 12, 67-67 (2012).

37. Koch, S. Home telehealth-Current state and future trends. International Journal of Medical Informatics 75, 565-576 (2006).

38. Krishna, S., Boren, S. A. \& Balas, E. A. Healthcare via Cell Phones: A Systematic Review. Telemedicine Journal and E-health 15, 231-240 (2009).

39. Trautmann, S., Rehm, J. \& Wittchen, H.-U. The economic costs of mental disorders. EMBO reports 17, 1245-1249 (2016). 
40. Firth, J. et al. Can smartphone mental health interventions reduce symptoms of anxiety? A meta-analysis of randomized controlled trials. Journal of Affective Disorders 218, 15-22 (2017).

41. Lüdtke, T., Pult, L. K., Schröder, J., Moritz, S. \& Bücker, L. A randomized controlled trial on a smartphone self-help application (Be Good to Yourself) to reduce depressive symptoms. Psychiatry Research-neuroimaging 269, 753-762 (2018).

42. Ly, K. H. et al. Smartphone-Supported versus Full Behavioural Activation for Depression: A Randomised Controlled Trial. PLOS ONE 10 (2015).

43. Proudfoot, J. G. Community Attitudes to the Appropriation of Mobile Phones for Monitoring and Managing Depression, Anxiety, and Stress. Journal of Medical Internet Research 12 (2010).

44. Andersson, E. et al. Long-term efficacy of Internet-based cognitive behavior therapy for obsessive-compulsive disorder with or without booster : a randomized controlled trial. Psychological Medicine 44, 2877-2887 (2014).

45. Marzano, L. et al. The application of mHealth to mental health: opportunities and challenges. The Lancet Psychiatry 2, 942-948 (2015).

46. Grist, R., Porter, J. \& Stallard, P. Mental health mobile apps for preadolescents and adolescents: a systematic review. Journal of Medical Internet Research 19 (2017).

47. Ehrenreich, B., Righter, B., Rocke, D. A., Dixon, L. \& Himelhoch, S. Are mobile phones and handheld computers being used to enhance delivery of psychiatric treatment? A systematic review. Journal of Nervous and Mental Disease 199, 886-891 (2011).

48. Donker, T. et al. Smartphones for smarter delivery of mental health programs: A systematic review. Journal of Medical Internet Research 15 (2013).

49. Naslund, J. A., Marsch, L. A., McHugo, G. J. \& Bartels, S. J. Emerging mHealth and eHealth interventions for serious mental illness: a review of the literature. Journal of Mental Health 24, 321-332 (2015).

50. Bateman, D. R. et al. Categorizing Health Outcomes and Efficacy of mHealth Apps for Persons With Cognitive Impairment: A Systematic Review. Journal of Medical Internet Research 19 (2017). 
51. Kazemi, D. M. et al. A Systematic Review of the mHealth Interventions to Prevent Alcohol and Substance Abuse. Journal of Health Communication 22, 413-432 (2017).

52. Berrouiguet, S., Baca-Garcia, E., Brandt, S. A., Walter, M. \& Courtet, P. Fundamentals for Future Mobile-Health (mHealth): A Systematic Review of Mobile Phone and Web-Based Text Messaging in Mental Health. Journal of Medical Internet Research 18 (2016).

53. Payne, H. E., Lister, C., West, J. H. \& Bernhardt, J. M. Behavioral Functionality of Mobile Apps in Health Interventions: A Systematic Review of the Literature. JMIR mHealth and uHealth 3 (2015).

54. Liberati, A. et al. The PRISMA statement for reporting systematic reviews and meta-analyses of studies that evaluate healthcare interventions: explanation and elaboration. BMJ 339 (2009).

55. Association., A. P. Diagnostic and statistical manual of mental disorders (DSM-5®) (2013).

56. For Standardization., I. O. Ergonomics of human system interaction - Part 210: Human-centered design for interactive systems (formerly known as 13407) (2009).

57. Granell, C. et al. Reproducibility Package for 'Smartphone apps for the treatment of mental disorders: a systematic review'. Zenodo (2019).

58. Prada, P. et al. EMOTEO: A Smartphone Application for Monitoring and Reducing Aversive Tension in Borderline Personality Disorder Patients, a Pilot Study. Perspectives in Psychiatric Care 53, 289-298. ISSN: 0031-5990 (2017).

59. Olbrich, H., Stengler, K. \& Olbrich, S. Smartphone based Geo-Feedback in obsessive compulsive disorder as facilitatory intervention: A case report. Journal of Obsessive-Compulsive and Related Disorders 8, 75-78 (2016).

60. Boisseau, C., Schwartzman, C., Lawton, J. \& Mancebo, M. App-guided exposure and response prevention for obsessive compulsive disorder: an open pilot trial. Cognitive Behaviour Therapy 46, 447-458 (2017).

61. Whiteside, S., Ale, C., Vickers Douglas, K., Tiede, M. \& Dammann, J. Case Examples of Enhancing Pediatric OCD Treatment with a Smartphone Application. Clinical Case Studies 13, 80-94 (2014). 
62. Horsch, C. et al. Mobile phone-delivered cognitive behavioral therapy for insomnia: A randomized waitlist controlled trial. Journal of Medical Internet Research 19 (2017).

63. Espie, C. A. et al. Digital Cognitive Behavioural Therapy for Insomnia versus sleep hygiene education: the impact of improved sleep on functional health, quality of life and psychological well-being. Study protocol for a randomised controlled trial. Trials 17. ISSN: 1745-6215 (2016).

64. Kyle, S. et al. Effects of digital Cognitive Behavioural Therapy for Insomnia on cognitive function: Study protocol for a randomised controlled trial. Trials 18 (2017).

65. Kolar, D., Hammerle, F., Jenetzky, E. \& Huss, M. Smartphone-Enhanced Low-Threshold Intervention for adolescents with Anorexia Nervosa (SELTIAN) waiting for outpatient psychotherapy: Study protocol of a randomised controlled trial. BMJ Open 7 (2017).

66. Tregarthen, J. P., Lock, J. \& Darcy, A. M. Development of a smartphone application for eating disorder self-monitoring. International Journal of Eating Disorders 48, 972-982. ISSN: 0276-3478 (Nov. 2015).

67. Nitsch, M. et al. A Guided Online and Mobile Self-Help Program for Individuals With Eating Disorders: An Iterative Engagement and Usability Study. Journal of Medical Internet Research 18, e7 (2016).

68. Zaffke, A. et al. iCanLearn: A Mobile Application for Creating Flashcards and Social Stories (TM) for Children with Autism in Smart Homes and Health Telematics 8456 (Springer Int. Publishing Ag., 2015), 225-230.

69. Skillen, K. .--L., Donnelly, M. P., Nugent, C. D. \& Booth, N. LifePal: A Mobile Self-management Tool for Supporting Young People with Autism in XIV Mediterranean Conference on Medical and Biological Engineering and Computing 201657 (Springer, 2016), 1168-1173.

70. Chacko, A., Isham, A., Cleek, A. F. \& McKay, M. M. Using mobile health technology to improve behavioral skill implementation through homework in evidence-based parenting intervention for disruptive behavior disorders in youth: study protocol for intervention development and evaluation. Pilot and feasibility studies 2, 57 (2016). 
71. Wenze, S., Armey, M., Weinstock, L., Gaudiano, B. \& Miller, I. An Open Trial of a Smartphone-assisted, Adjunctive Intervention to Improve Treatment Adherence in Bipolar Disorder. Journal of Psychiatric Practice 22, 492-504 (2016).

72. Depp, C. et al. Augmenting psychoeducation with a mobile intervention for bipolar disorder: A randomized controlled trial. Journal of Affective Disorders 174, 23-30 (2015).

73. Hidalgo-Mazzei, D., Vieta, E. \& Colom, F. Self-monitoring and psychoeducation in bipolar patients with a smart-phone application (SIMPLe) project: Preliminary results from a feasibility study in MOBIHEALTH 2015 - 5th EAI International Conference on Wireless Mobile Communication and Healthcare - Transforming Healthcare through Innovations in Mobile and Wireless Technologies (2015).

74. Hidalgo-Mazzei, D. et al. Psychoeducation in bipolar disorder with a SIMPLe smartphone application: Feasibility, acceptability and satisfaction. Journal of Affective Disorders 200, 58-66 (2016).

75. Hidalgo-Mazzei, D. et al. Is a SIMPLe smartphone application capable of improving biological rhythms in bipolar disorder? Journal of Affective Disorders 223, 10-16. ISSN: 0165-0327 (2017).

76. Muroff, J. et al. Use of a Smartphone Recovery Tool for Latinos with CoOccurring Alcohol and Other Drug Disorders and Mental Disorders. Journal of Dual Diagnosis 13, 280-290 (2017).

77. Tajima-Pozo, K., Ruiz-Manrique, G. \& Montanes, F. Treating enuresis in a patient with ADHD: Application of a novel behavioural modification therapy. BMJ Case Reports (2014).

78. Vilardaga, R. et al. User-centered design of learn to quit, a smoking cessation smartphone app for people with serious mental illness. Journal of Medical Internet Research 20 (2018).

79. Minami, H. et al. Rationale, design and pilot feasibility results of a smartphoneassisted, mindfulness-based intervention for smokers with mood disorders: Project mSMART MIND. Contemporary Clinical Trials 66, 36-44 (2018). 
80. Benarous, X. et al. Ecological Momentary Assessment and Smartphone Application Intervention in Adolescents with Substance Use and Comorbid Severe Psychiatric Disorders: Study Protocol. Frontiers in psychiatry 7, 157 (2016).

81. Matalenas, L., McLaughlin, A., Chen, Y. \& Daughters, S. Developing a smartphone application for the Life Enhancement Treatment for Substance Use (LETS act): Designing for motivation and feedback in Proceedings of the Human Factors and Ergonomics Society 2015-January (2015), 11001104.

82. Tighe, J. et al. Ibobbly mobile health intervention for suicide prevention in Australian Indigenous youth: A pilot randomised controlled trial. BMJ Open 7 (2017).

83. Bush, N. et al. A Virtual Hope Box: Randomized controlled trial of a smartphone app for emotional regulation and coping with distress. Psychiatric Services 68, 330-336 (2017).

84. Shand, F., Ridani, R., Tighe, J. \& Christensen, H. The effectiveness of a suicide prevention app for indigenous Australian youths: Study protocol for a randomized controlled trial. Trials 14 (2013).

85. O'Brien, K. H. M., LeCloux, M., Ross, A., Gironda, C. \& Wharff, E. A. A pilot study of the acceptability and usability of a smartphone application intervention for suicidal adolescents and their parents. Archives of Suicide Research 21, 254-264 (2017).

86. Stallard, P., Porter, J. \& Grist, R. Safety, Acceptability, and Use of a Smartphone App, Bluelce, for Young People Who Self-Harm: Protocol for an Open Phase I Trial. JMIR Research Protocols 5. ISSN: 1929-0748 (2016).

87. Stallard, P., Porter, J. \& Grist, R. A Smartphone App (Bluelce) for Young People Who Self-Harm: Open Phase 1 Pre-Post Trial. JMIR mHealth and uHealth 6, e32 (June 2018).

88. Grist, R., Porter, J. \& Stallard, P. Acceptability, Use, and Safety of a Mobile Phone App (Bluelce) for Young People Who Self-Harm: Qualitative Study of Service Users' Experience. JMIR Mental Health 5, e16 (Feb. 2018). 
89. Bucci, S. et al. Using mobile technology to deliver a cognitive behaviour therapy-informed intervention in early psychosis (Actissist): Study protocol for a randomised controlled trial. Trials 16 (2015).

90. Kim, S.-W. et al. Development and feasibility of smartphone application for cognitive-behavioural case management of individuals with early psychosis. Early intervention in psychiatry (May 2017).

91. De Almeida, R. et al. Development of a hybrid application for psychotic disorders self-management. Advances in Intelligent Systems and Computing 615, 229-237 (2017).

92. Schlosser, D. et al. Feasibility of PRIME: A Cognitive Neuroscience-Informed Mobile App Intervention to Enhance Motivated Behavior and Improve Quality of Life in Recent Onset Schizophrenia. JMIR Research Protocols 5. ISSN: 1929-0748 (2016).

93. Garety, P. et al. SlowMo, a digital therapy targeting reasoning in paranoia, versus treatment as usual in the treatment of people who fear harm from others: Study protocol for a randomised controlled trial. Trials 18 (2017).

94. Husain, N. et al. TechCare: mobile assessment and therapy for psychosis - an intervention for clients in the Early Intervention Service: A feasibility study protocol. SAGE open medicine 4, 2050312116669613 (2016).

95. Ben-Zeev, D. et al. Development and usability testing of FOCUS: A smartphone system for self-management of schizophrenia. Psychiatric Rehabilitation Journal 36, 289-296 (2013).

96. Ben-Zeev, D. et al. Feasibility, acceptability, and preliminary efficacy of a smartphone intervention for schizophrenia. Schizophrenia Bulletin 40, 1244-1253 (2014).

97. Leonard, N. R. et al. Mobile Health Technology Using a Wearable Sensorband for Female College Students With Problem Drinking: An Acceptability and Feasibility Study. JMIR mHealth and uHealth 5, e90 (July 2017).

98. Quanbeck, A. et al. Implementing a Mobile Health System to Integrate the Treatment of Addiction Into Primary Care: A Hybrid ImplementationEffectiveness Study. Journal of Medical Internet Research 20. ISSN: 14388871 (2018). 
99. Zeng, E., Vilardaga, R., Heffner, J., Mull, K. \& Bricker, J. Predictors of Utilization of a Novel Smoking Cessation Smartphone App. Telemedicine and e-Health 21, 998-1004 (2015).

100. Alsharif, A. \& Philip, N. Cognitive behavioural therapy embedding smoking cessation program using smart phone Technologies in Proceedings of the 2015 5th World Congress on Information and Communication Technologies, WICT 2015 (2016), 134-139.

101. Dennis, M., Scott, C., Funk, R. \& Nicholson, L. A Pilot Study to Examine the Feasibility and Potential Effectiveness of Using Smartphones to Provide Recovery Support for Adolescents. Substance Abuse 36, 486-492 (2015).

102. Scott, C., Dennis, M. \& Gustafson, D. Using smartphones to decrease substance use via self-monitoring and recovery support: Study protocol for a randomized control trial. Trials 18 (2017).

103. Mellentin, A., Nielsen, B., Nielsen, A., Yu, F. \& Stenager, E. A randomized controlled study of exposure therapy as aftercare for alcohol use disorder: Study protocol. BMC Psychiatry 16 (2016).

104. Mellentin, A. I., Stenager, E., Nielsen, B., Nielsen, A. S. \& Yu, F. A Smarter Pathway for Delivering Cue Exposure Therapy? The Design and Development of a Smartphone App Targeting Alcohol Use Disorder. JMIR mHealth and uHealth 5. ISSN: 2291-5222 (2017).

105. Businelle, M. et al. An ecological momentary intervention for smoking cessation: Evaluation of feasibility and effectiveness. Journal of Medical Internet Research 18 (2016).

106. Hébert, E. et al. An ecological momentary intervention for smoking cessation: The associations of just-in-time, tailored messages with lapse risk factors. Addictive Behaviors 78, 30-35 (2018).

107. Morland, L. et al. Using a mobile application in the management of anger problems among veterans: A pilot study. Military Medicine 181, 990-995 (2016).

108. Roy, M. J., Highland, K. B. \& Costanzo, M. A. GETSmart: Guided education and training via smart phones to promote resilience. Annual Review of CyberTherapy and Telemedicine 13, 123-128 (2015). 
109. Roy, M. et al. An App a Day Keeps the Doctor Away: Guided Education and Training via Smartphones in Subthreshold Post Traumatic Stress Disorder. Cyberpsychology, Behavior, and Social Networking 20, 470-478 (2017).

110. Possemato, K. et al. Using PTSD Coach in primary care with and without clinician support: A pilot randomized controlled trial. General Hospital Psychiatry 38, 94-98 (2016).

111. Mackintosh, M.-A. et al. Using a mobile application in the treatment of dysregulated anger among veterans. Military Medicine 182, e1941-e1949 (2017).

112. Kuhn, E. et al. Clinician Perceptions of Using a Smartphone App with Prolonged Exposure Therapy. Administration and Policy in Mental Health and Mental Health Services Research 41, 800-807. ISSN: 0894-587X (Nov. 2014).

113. Kuhn, E. et al. Clinician Characteristics and Perceptions Related to Use of the PE (Prolonged Exposure) Coach Mobile App. Professional PsychologyResearch and Practice 46, 437-443. ISSN: 0735-7028 (Dec. 2015).

114. Reger, G. M., Skopp, N. A., Edwards-Stewart, A. \& Lemus, E. L. Comparison of Prolonged Exposure (PE) Coach to Treatment as Usual: A Case Series With Two Active Duty Soldiers. Military Psychology 27, 287-296. ISSN: 0899-5605 (Sept. 2015).

115. Reger, G. et al. Barriers and facilitators to mobile application use during PTSD treatment: Clinician adoption of PE coach. Professional Psychology: Research and Practice 48, 510-517 (2017).

116. Owen, J. E. et al. mHealth in the Wild: Using Novel Data to Examine the Reach, Use, and Impact of PTSD Coach. JMIR Mental Health 2. ISSN: 2368-7959 (Mar. 2015).

117. Miner, A. et al. Feasibility, acceptability, and potential efficacy of the PTSD coach app: A pilot randomized controlled trial with community trauma survivors. Psychological Trauma: Theory, Research, Practice, and Policy 8, 384-392 (2016).

118. Kuhn, E. et al. A randomized controlled trial of a smartphone app for posttraumatic stress disorder symptoms. Journal of Consulting and Clinical Psychology 85, 267-273 (2017). 
119. Possemato, K., Kuhn, E., Johnson, E., Hoffman, J. \& Brooks, E. Development and refinement of a clinician intervention to facilitate primary care patient use of the PTSD Coach app. Translational Behavioral Medicine 7, 116-126 (2017).

120. Levin, M., Haeger, J., Pierce, B. \& Cruz, R. Evaluating an Adjunctive Mobile App to Enhance Psychological Flexibility in Acceptance and Commitment Therapy. Behavior Modification 41, 846-867 (2017).

121. Mistler, L. A., Ben-Zeev, D., Carpenter-Song, E., Brunette, M. F. \& Friedman, M. J. Mobile Mindfulness Intervention on an Acute Psychiatric Unit: Feasibility and Acceptability Study. JMIR Mental Health 4. ISSN: 2368-7959 (2017).

122. Povey, J. et al. Acceptability of Mental Health Apps for Aboriginal and Torres Strait Islander Australians: A Qualitative Study. Journal of Medical Internet Research 18. ISSN: 1438-8871 (2016).

123. Mohr, D. et al. Intellicare: An eclectic, skills-based app suite for the treatment of depression and anxiety. Journal of Medical Internet Research 19 (2017).

124. Addepally, S. \& Purkayastha, S. Mobile-application based cognitive behavior therapy (CBT) for identifying and managing depression and anxiety. Lecture Notes in Computer Science (including subseries Lecture Notes in Artificial Intelligence and Lecture Notes in Bioinformatics) 10287 LNCS, 3-12 (2017).

125. Proudfoot, J. et al. Impact of a mobile phone and web program on symptom and functional outcomes for people with mild-to-moderate depression, anxiety and stress: A randomised controlled trial. BMC Psychiatry 13 (2013).

126. Fortuna, K. L. et al. Feasibility, Acceptability, and Preliminary Effectiveness of a Peer-Delivered and Technology Supported Self-Management Intervention for Older Adults with Serious Mental IIIness. The Psychiatric quarterly (Sept. 2017).

127. Pramana, G., Parmanto, B., Kendall, P. C. \& Silk, J. S. The SmartCAT: An $\mathrm{m}$ - Health Platform for Ecological Momentary Intervention in Child Anxiety Treatment. Telemedicine and e-Health 20, 419-427. ISSN: 1530-5627 (May 2014). 
128. Bauer, A. et al. Lessons from the deployment of the spirit app to support Collaborative care for rural patients with complex psychiatric conditions in UbiComp/ISWC 2017 - Adjunct Proceedings of the 2017 ACM International Joint Conference on Pervasive and Ubiquitous Computing and Proceedings of the 2017 ACM International Symposium on Wearable Computers (2017), 772-780.

129. Huang, S.-Y., Sano, A. \& Kwan, C. The moment: A mobile tool for people with depression or bipolar disorder in UbiComp 2014 - Adjunct Proceedings of the 2014 ACM International Joint Conference on Pervasive and Ubiquitous Computing (2014), 235-238.

130. Fleming, J. B., Hill, Y. N. \& Burns, M. N. Usability of a Culturally Informed mHealth Intervention for Symptoms of Anxiety and Depression: Feedback From Young Sexual Minority Men. JMIR Human Factors 4, e22 (Aug. 2017).

131. Whiteman, K. L., Lohman, M. C., Gill, L. E., Bruce, M. L. \& Bartels, S. J. Adapting a Psychosocial Intervention for Smartphone Delivery to MiddleAged and Older Adults with Serious Mental Illness. American Journal of Geriatric Psychiatry 25, 819-828. ISSN: 1064-7481 (2017).

132. Nicholson, J., Wright, S. M. \& Carlisle, A. M. Pre-post, mixed-methods feasibility study of the WorkingWell mobile support tool for individuals with serious mental illness in the USA: a pilot study protocol. BMJ open 8, e019936 (2018).

133. Christoforou, M., Fonseca, J. A. S. \& Tsakanikos, E. Two Novel Cognitive Behavioral Therapy-Based Mobile Apps for Agoraphobia: Randomized Controlled Trial. Journal of Medical Internet Research 19. ISSN: 1438-8871 (2017).

134. Ivanova, E. et al. Guided and unguided Acceptance and Commitment Therapy for social anxiety disorder and/or panic disorder provided via the Internet and a smartphone application: A randomized controlled trial. Journal of Anxiety Disorders 44, 27-35 (2016).

135. Carper, M. M. Multimedia Field Test Thinking About Exposures? There's an App for That! Cognitive and behavioral practice 24, 121-127 (Feb. 2017). 
136. Michelle, T., Jarzabek, S. \& Wadhwa, B. CBT Assistant: MHealth App for psychotherapy in 2014 IEEE Global Humanitarian Technology Conference South Asia Satellite, GHTC-SAS 2014 (2014), 135-140.

137. Szigethy, E. et al. A study protocol for a non-randomised comparison trial evaluating the feasibility and effectiveness of a mobile cognitive-behavioural programme with integrated coaching for anxious adults in primary care. BMJ open 8, e019108 (2018).

138. Clough, B. \& Casey, L. Therapy on the move: The development of a therapeutic smartphone application. International Journal of Cyber Behavior, Psychology and Learning 5, 33-41 (2015).

139. Stupar-Rutenfrans, S., Ketelaars, L. E. H. \& van Gisbergen, M. S. Beat the Fear of Public Speaking: Mobile 360 degrees Video Virtual Reality Exposure Training in Home Environment Reduces Public Speaking Anxiety. Cyberpsychology Behavior and Social Networking 20, 624-633. ISSN: 21522715 (2017).

140. Stegemann, S., Ebenfeld, L., Lehr, D., Berking, M. \& Funk, B. Development of a mobile application for people with panic disorder as augmentation for an internet-based intervention in 2013 Federated Conference on Computer Science and Information Systems, FedCSIS 2013 (2013), 1331-1337.

141. Ebenfeld, L. et al. Efficacy of a hybrid online training for panic symptoms and agoraphobia: Study protocol for a randomized controlled trial. Trials 15 (2014).

142. Lindner, P., Ivanova, E., Ly, K., Andersson, G. \& Carlbring, P. Guided and unguided $\mathrm{CBT}$ for social anxiety disorder and/or panic disorder via the Internet and a smartphone application: Study protocol for a randomised controlled trial. Trials 14 (2013).

143. Repetto, C. et al. Virtual reality and mobile phones in the treatment of generalized anxiety disorders: A phase-2 clinical trial. Personal and Ubiquitous Computing 17, 253-260 (2013).

144. Cruz, L. et al. A wearable and mobile intervention delivery system for individuals with panic disorder in ACM International Conference Proceeding Series 30-November-2015 (2015), 175-182. 
145. Benton, S., Heesacker, M., Snowden, S. \& Lee, G. Therapist-Assisted, Online (TAO) intervention for anxiety in college students: TAO outperformed treatment as usual. Professional Psychology: Research and Practice $\mathbf{4 7}$ (2016).

146. Kumar, S., Bell, M. \& Juusola, J. Mobile and traditional cognitive behavioral therapy programs for generalized anxiety disorder: A cost-effectiveness analysis. PLOS ONE 13 (2018).

147. Baumel, A., Tinkelman, A., Mathur, N. \& Kane, J. Digital peer-support platform (7Cups) as an adjunct treatment for women with postpartum depression: Feasibility, acceptability, and preliminary efficacy study. Journal of Medical Internet Research 20 (2018).

148. Giosan, C. et al. Reducing depressive symptomatology with a smartphone app: Study protocol for a randomized, placebo-controlled trial. Trials $\mathbf{1 8}$ (2017).

149. Watts, S. et al. CBT for depression: a pilot RCT comparing mobile phone vs. computer. BMC Psychiatry 13. ISSN: 1471-244X (Feb. 2013).

150. Mohr, D. et al. MedLink: A mobile intervention to address failure points in the treatment of depression in general medicine in Proceedings of the 2015 9th International Conference on Pervasive Computing Technologies for Healthcare, PervasiveHealth 2015 (2015), 100-107.

151. Wahle, F., Kowatsch, T., Fleisch, E., Rufer, M. \& Weidt, S. Mobile Sensing and Support for People With Depression: A Pilot Trial in the Wild. JMIR mHealth and uHealth 4. ISSN: 2291-5222 (Sept. 2016).

152. Birney, A. J., Gunn, R., Russell, J. K. \& Ary, D. V. MoodHacker Mobile Web App With Email for Adults to Self-Manage Mild-to-Moderate Depression: Randomized Controlled Trial. JMIR mHealth and uHealth 4, 100-118. ISSN: 2291-5222 (2016).

153. Dahne, J. et al. Moodivate: A self-help behavioral activation mobile app for utilization in primary care-Development and clinical considerations. International Journal of Psychiatry in Medicine 52, 160-175 (2017). 
154. Gordon, M., Henderson, R., Holmes, J., Wolters, M. \& Bennett, I. Participatory design of eHealth solutions for women from vulnerable populations with perinatal depression. Journal of the American Medical Informatics Association 23, 105-109 (2016).

155. Schlosser, D. et al. The feasibility, acceptability, and outcomes of PRIMED: A novel mobile intervention treatment for depression. Depression and Anxiety 34, 546-554 (2017).

156. Takahashi, K., Takada, K. \& Hirao, K. Feasibility and preliminary efficacy of a smartphone application intervention for subthreshold depression. Early intervention in psychiatry (June 2018).

157. Roepke, A. et al. Randomized Controlled Trial of SuperBetter, a SmartphoneBased/Internet-Based Self-Help Tool to Reduce Depressive Symptoms. Games for Health Journal 4, 235-246 (2015).

158. Stiles-Shields, C. et al. Exploring User Learnability and Learning Performance in an App for Depression: Usability Study. JMIR Human Factors 4, e18 (Aug. 2017).

159. Hur, J.-W., Kim, B., Park, D. \& Choi, S.-W. A Scenario-Based Cognitive Behavioral Therapy Mobile App to Reduce Dysfunctional Beliefs in Individuals with Depression: A Randomized Controlled Trial. Telemedicine journal and e-health : the official journal of the American Telemedicine Association (Jan. 2018).

160. Watanabe, N. et al. Adding smartphone-based cognitive-behavior therapy to pharmacotherapy for major depression (FLATT project): study protocol for a randomized controlled trial. Trials 16. ISSN: 1745-6215 (July 2015).

161. Mantani, A. et al. Smartphone cognitive behavioral therapy as an adjunct to pharmacotherapy for refractory depression: Randomized controlled trial. Journal of Medical Internet Research 19 (2017).

162. Furukawa, T. et al. Cognitive and behavioral skills exercises completed by patients with major depression during smartphone cognitive behavioral therapy: Secondary analysis of a randomized controlled trial. Journal of Medical Internet Research 20 (2018). 
163. Ly, K. et al. Behavioural activation versus mindfulness-based guided selfhelp treatment administered through a smartphone application: A randomised controlled trial. BMJ Open 4 (2014).

164. Hung, G.-L., Yang, P.-C., Wang, C.-Y. \& Chiang, J.-H. A smartphone-based personalized activity recommender system for patients with depression in MOBIHEALTH 2015 - 5th EAI International Conference on Wireless Mobile Communication and Healthcare - Transforming Healthcare through Innovations in Mobile and Wireless Technologies (2015).

165. Ly, K. et al. Smartphone-supported versus full behavioural activation for depression: A randomised controlled trial. PLOS ONE 10 (2015).

166. Anguera, J., Jordan, J., Castaneda, D., Gazzaley, A. \& Areán, P. Conducting a fully mobile and randomised clinical trial for depression: Access, engagement and expense. BMJ Innovations 2, 14-21 (2016).

167. Giosan, C. et al. Using a smartphone app to reduce cognitive vulnerability and mild depressive symptoms: Study protocol of an exploratory randomized controlled trial. Trials 17 (2016).

168. Yoo, J.-S., Kim, I.-S. \& Gwon, J.-A. Design and implementation of smartphone application for measurement and management of depressive emotions in adolescents using cognitive behavioral therapy. Communications in Computer and Information Science 714, 317-323 (2017).

169. Martín-Jurado, A., de la Gándara Martín, J., Carbajo, S. C., Hernández, A. M. \& Sánchez-Hernández, J. Análisis de concordancia de las derivaciones de atención primaria a salud mental. Semergen - Medicina De Familia 38, 354-359 (2012).

170. Griffiths, K. M. \& Christensen, H. Internet-based mental health programs: A powerful tool in the rural medical kit. Australian Journal of Rural Health 15, 81-87 (2007).

171. Kazdin, A. E. Technology-Based Interventions and Reducing the Burdens of Mental Illness: Perspectives and Comments on the Special Series. Cognitive and Behavioral Practice 22, 359-366 (2015).

172. Kazdin, A. E. \& Blase, S. L. Rebooting Psychotherapy Research and Practice to Reduce the Burden of Mental Illness. Perspectives on Psychological Science 6, 21-37 (2011). 
173. Andrews, G. et al. Computer therapy for the anxiety and depression disorders is effective, acceptable and practical health care: An updated metaanalysis. Journal of Anxiety Disorders 55, 70-78 (2018).

174. Richards, D. \& Richardson, T. Computer-based psychological treatments for depression: a systematic review and meta-analysis. Clinical Psychology Review 32, 329-342 (2012).

175. Mendlowicz, M. V. \& Stein, M. B. Quality of Life in Individuals With Anxiety Disorders. American Journal of Psychiatry 157, 669-682 (2000).

176. Rapaport, M. H., Clary, C., Fayyad, R. \& Endicott, J. Quality-of-Life Impairment in Depressive and Anxiety Disorders. American Journal of Psychiatry 162, 1171-1178 (2005).

177. Andlin-Sobocki, P. \& Wittchen, H.-U. Cost of anxiety disorders in Europe. European Journal of Neurology 12, 39-44 (2005).

178. Cuijpers, P., Beekman, A. T. F. \& Reynolds, C. F. Preventing Depression: A Global Priority. JAMA 307, 1033-1034 (2012).

179. Kessler, R. C. et al. Lifetime Prevalence and Age-of-Onset Distributions of DSM-IV Disorders in the National Comorbidity Survey Replication. Archives of General Psychiatry 62, 593-602 (2005).

180. Kessler, R. C., Chiu, W. T., Demler, O. \& Walters, E. E. Prevalence, Severity, and Comorbidity of 12-Month DSM-IV Disorders in the National Comorbidity Survey Replication. Archives of General Psychiatry 62, 617-627 (2005).

181. Gunderson, J. G. Borderline Personality Disorder: Ontogeny of a Diagnosis. American Journal of Psychiatry 166, 530-539 (2009).

182. Lenzenweger, M. F., Lane, M. C., Loranger, A. W. \& Kessler, R. C. DSMIV personality disorders in the National Comorbidity Survey Replication. Biological Psychiatry 62, 553-564 (2007).

183. Carvalho, M. d., Guimarães, H., Ferreira, J. \& Freitas, A. Intention to use M-learning: An extension of the technology acceptance model in 19th International Conference on Recent Advances in Retailing and Consumer Services Science (2012), 9-12. 
184. Matthews, M., Abdullah, S. \& Choudhury, T. Tracking Mental Well-Being: Balancing Rich Sensing and Patient Needs. IEEE Computer 47, 36-43 (2014).

185. Jeong, S. \& Breazeal, C. Toward Robotic Companions that Enhance Psychological Wellbeing with Smartphone Technology in Proceedings of the Companion of the 2017 ACM/IEEE International Conference on HumanRobot Interaction (2017), 345-346.

186. Cella, M. et al. Using wearable technology to detect the autonomic signature of illness severity in schizophrenia. Schizophrenia Research 195, 537-542 (2017).

187. Hodges, S., Berry, E. \& Wood, K. SenseCam: A wearable camera that stimulates and rehabilitates autobiographical memory. Memory 19, 685696 (2011).

188. Huang, T.-L. \& Liao, S. A model of acceptance of augmented-reality interactive technology: the moderating role of cognitive innovativeness. Electronic Commerce Research 15, 269-295 (2015).

189. World Health Organization. Depression and other common mental disorders: global health estimates tech. rep. (2017).

190. Twenge, J. M. Have smartphones destroyed a generation? The Atlantic 3. https : / /www . theatlantic . com/magazine/archive/2017/09/has - the smartphone-destroyed-a-generation/534198/ (2017).

191. Auerbach, R. P. et al. WHO world mental health surveys international college student project: Prevalence and distribution of mental disorders. Journal of Abnormal Psychology 127, 623 (2018).

192. Wykes, T. et al. Mental health research priorities for Europe. The Lancet Psychiatry 2, 1036-1042 (2015).

193. Lary, D., Woolf, S., Faruque, F. \& LePage, J. Holistics 3.0 for Health. ISPRS International Journal of Geo-Information 3, 1023-1038. ISSN: 2220-9964 (2014).

194. Lopez, R. P. The Built Environment and Public Health 1st (John Wiley \& Sons: Hoboken, USA, 2012). 
195. McAndrew, F. T. Environmental psychology. (Thomson Brooks/Cole Publishing Co, 1993).

196. Rana, R., Hume, M., Reilly, J., Jurdak, R. \& Soar, J. Opportunistic and context-aware affect sensing on smartphones. IEEE Pervasive Computing, 60-69 (2016).

197. Kay, M., Santos, J. \& Takane, M. mHealth: New horizons for health through mobile technologies. World Health Organization 64, 66-71 (2011).

198. Lindhiem, O., Bennett, C. B., Rosen, D. \& Silk, J. Mobile technology boosts the effectiveness of psychotherapy and behavioral interventions: a metaanalysis. Behavior modification 39, 785-804 (2015).

199. Possemato, K., Kuhn, E., Johnson, E. M., Hoffman, J. E. \& Brooks, E. Development and refinement of a clinician intervention to facilitate primary care patient use of the PTSD Coach app. Translational behavioral medicine 7, 116-126 (2016).

200. Wac, K., Fiordelli, M., Gustarini, M. \& Rivas, H. Quality of life technologies: Experiences from the field and key challenges. IEEE Internet Computing 19, 28-35 (2015).

201. Luxton, D. D., McCann, R. A., Bush, N. E., Mishkind, M. C. \& Reger, G. M. mHealth for mental health: Integrating smartphone technology in behavioral healthcare. Professional Psychology: Research and Practice 42, 505 (2011).

202. Gravenhorst, F. et al. Mobile phones as medical devices in mental disorder treatment: an overview. Personal and Ubiquitous Computing 19, 335-353 (2015).

203. Ebert, D. D., Cuijpers, P., Muñoz, R. F. \& Baumeister, H. Prevention of mental health disorders using internet-and mobile-based interventions: a narrative review and recommendations for future research. Frontiers in psychiatry $\mathbf{8}$, 116 (2017).

204. Ferrás, C., Garcıa, Y., Aguilera, A. \& Rocha, Á. How can geography and mobile phones contribute to psychotherapy? Journal of medical systems 41, 92 (2017).

205. Palmius, N. et al. Detecting bipolar depression from geographic location data. IEEE Transactions on Biomedical Engineering 64, 1761-1771 (2017). 
206. Saeb, S., Lattie, E. G., Kording, K. P. \& Mohr, D. C. Mobile Phone Detection of Semantic Location and Its Relationship to Depression and Anxiety. JMIR mHealth and uHealth 5 (2017).

207. Saeb, S., Lattie, E. G., Schueller, S. M., Kording, K. P. \& Mohr, D. C. The relationship between mobile phone location sensor data and depressive symptom severity. PeerJ 4, e2537 (2016).

208. Buschmann, F., Meunier, R., Rohnert, H., Sommerlad, P. \& Stal, M. PatternOriented Software Architecture - Volume 1: A System of Patterns (Wiley Publishing, 1996).

209. Gamma, E., Helm, R., Johnson, R. \& Vlissides, J. Design Patterns: Elements of Reusable Object-oriented Software (Addison-Wesley Longman Publishing Co., Inc., Boston, MA, USA, 1995).

210. Díaz, L., Granell, C., Huerta, J. \& Gould, M. Web 2.0 Broker: A standardsbased service for spatio-temporal search of crowd-sourced information. Applied Geography 35, 448-459. ISSN: 0143-6228 (2012).

211. Nativi, S., Craglia, M. \& Pearlman, J. Earth Science Infrastructures Interoperability: The Brokering Approach. IEEE Journal of Selected Topics in Applied Earth Observations and Remote Sensing 6, 1118-1129 (2013).

212. Glaser, B. G. \& Strauss, A. L. The Discovery of Grounded Theory: Strategies for Qualitative Research 1st (AldineTrasaction: New Brunswick, USA, 1967).

213. Strauss, A. L. \& Corbin, J. Basics of Qualitative Research: Techniques and Procedures for Developing Grounded Theory 2nd (SAGE: USA, 1998).

214. Babbie, E. The Practice of Social Research 12th (Cengage Learning: Wadsworth, USA, 2009).

215. Huitt, W., Hummel, J. \& Kaeck, D. Assessment, measurement, evaluation, and research. Educational Psychology Interactive (2001).

216. Bowen, G. A. Document Analysis as a Qualitative Research Method. Qualitative Research Journal 9, 27-40 (2009).

217. Ostermann, F. O. \& Granell, C. Advancing science with VGI: Reproducibility and replicability of recent studies using VGI. Transactions in GIS 21, 224237 (2017). 
218. González-Bailón, S. Decoding the Social World: Data Science and the Unintended Consequences of Communication (MIT Press, 2017).

219. Patton, M. Q. Qualitative Research \& Evaluation Methods 4th (Sage: Thousand Oaks, USA, 2015).

220. Osma, J., Sprenger, M. \& Mettler, T. Introduction of e-mental health in national health systems $-\mathrm{A}$ health professionals' perspective. Health Policy and Technology 6, 436-445 (2017).

221. Martínez-Pérez, B., de la Torre-Díez, I., López-Coronado, M. \& HerrerosGonzález, J. Mobile Apps in Cardiology: Review. JMIR mHealth and uHealth 1, e15 (July 2013).

222. Van Ameringen, M., Turna, J., Khalesi, Z., Pullia, K. \& Patterson, B. There is an app for that! The current state of mobile applications (apps) for DSM-5 obsessive-compulsive disorder, posttraumatic stress disorder, anxiety and mood disorders. Depression and anxiety 34, 526-539 (2017).

223. Tay, J. L., Tay, Y. F. \& Klainin-Yobas, P. Effectiveness of information and communication technologies interventions to increase mental health literacy: A systematic review. Early intervention in psychiatry (2018).

224. Wilkinson, N., Ang, R. P. \& Goh, D. H. Online video game therapy for mental health concerns: a review. International journal of social psychiatry 54, 370-382 (2008).

225. BinDhim, N. F. et al. Depression screening via a smartphone app: crosscountry user characteristics and feasibility. Journal of the American Medical Informatics Association 22, 29-34 (2014).

226. Heron, K. E. \& Smyth, J. M. Ecological momentary interventions: incorporating mobile technology into psychosocial and health behaviour treatments. British journal of health psychology 15, 1-39 (2010).

227. Haggerty, E. Healthcare and digital transformation. Network Security 2017, 7-11. ISSN: 1353-4858 (2017).

228. Bohnsack, R., Hanelt, A., Marz, D. \& Antunes, C. Old Wine in New Bottles? A Systematic Review of the Literature on Digital Transformation. Academy of Management Global Proceedings, 197 (2018). 
229. Clarke, G. \& Yarborough, B. J. Evaluating the promise of health IT to enhance/expand the reach of mental health services. General hospital psychiatry 35, 339-344 (2013).

230. Hamari, J. \& Keronen, L. Why do people play games? A meta-analysis. International Journal of Information Management 37, 125-141 (2017).

231. Li, J., Theng, Y.-L. \& Foo, S. Game-based digital interventions for depression therapy: a systematic review and meta-analysis. Cyberpsychology, Behavior, and Social Networking 17, 519-527 (2014).

232. Deterding, S., Dixon, D., Khaled, R. \& Nacke, L. From game design elements to gamefulness: defining gamification in Proceedings of the 15th international academic MindTrek conference: Envisioning future media environments (2011), 9-15.

233. Spallazzo, D. \& Mariani, I. Location-Based Mobile Games: Design Perspectives (Springer, 2018).

234. Birk, M. V., Vanden Abeele, V., Wadley, G. \& Torous, J. Forum on Video Games for Mental Health in Proceedings of the 2018 Annual Symposium on Computer-Human Interaction in Play Companion Extended Abstracts (ACM, 2018), 683-688.

235. Miralles, I., Granell, C., Rodrıguez-Pupo, L. E., Casteleyn, S. \& Huerta, J. Games, Health and the City: Developing Location-Aware Games for Leveraging the Most Suitable Places for Physical Activity in Extended Abstracts Publication of the Annual Symposium on Computer-Human Interaction in Play (ACM, Amsterdam, The Netherlands, 2017), 239-245.

236. Owen, N., Humpel, N., Leslie, E., Bauman, A. \& Sallis, J. F. Understanding environmental influences on walking: review and research agenda. American journal of preventive medicine 27, 67-76 (2004).

237. De Bourdeaudhuij, I., Sallis, J. F. \& Saelens, B. E. Environmental correlates of physical activity in a sample of Belgian adults. American journal of health promotion 18, 83-92 (2003).

238. Elwood, S. Geographic Information Science: new geovisualization technologies-emerging questions and linkages with GIScience research. Progress in Human Geography 33, 256-263 (2009). 
239. Blaschke, T. \& Merschdorf, H. Geographic information science as a multidisciplinary and multiparadigmatic field. Cartography and Geographic Information Science 41, 196-213 (2014).

240. Pile, S. Emotions and affect in recent human geography. Transactions of the Institute of British Geographers 35, 5-20 (2010).

241. Ahlqvist, O. \& Schlieder, C. Introducing Geogames and Geoplay: Characterizing an Emerging Research Field in Geogames and Geoplay (Springer, 2018), 1-18.

242. Rodríguez-Pupo, L. E., Casteleyn, S. \& Granell, C. On Metrics for LocationAware Games. ISPRS International Journal of Geo-Information 6 (2017).

243. Kuhn, W. Core concepts of spatial information for transdisciplinary research. International Journal of Geographical Information Science 26, 2267-2276 (2012).

244. Kuhn, W. \& Ballatore, A. Designing a language for spatial computing in AGILE 2015 (Springer, 2015), 309-326.

245. Janelle, D. G. \& Goodchild, M. F. Concepts, principles, tools, and challenges in spatially integrated social science in The SAGE handbook of GIS and society (SAGE Publications Ltd. London: SAGE Publications Ltd., 2011), 27-45.

246. Sintoris, C. Extracting game design patterns from game design workshops. International Journal of Intelligent Engineering Informatics 3, 166-185 (2015).

247. Randell, D. A., Cui, Z. \& Cohn, A. G. A spatial logic based on regions and connection. in Principles of Knowledge Representation and Reasoning (KR-92) (Morgan Kaufmann Publishers, 1992), 165-176.

248. Egenhofer, M. J., Clementini, E. \& Di Felice, P. Topological relations between regions with holes. International Journal of Geographical Information Science 8, 129-142 (1994).

249. Lü, G. et al. Reflections and speculations on the progress in Geographic Information Systems (GIS): a geographic perspective. International Journal of Geographical Information Science 33, 346-367 (2018). 
250. Stedman, R. C. Is it really just a social construction?: The contribution of the physical environment to sense of place. Society \& Natural Resources 16, 671-685 (2003).

251. Tuan, Y.-F. Space and place: The perspective of experience (University of Minnesota Press, 1977).

252. Duff, C. On the role of affect and practice in the production of place. Environment and Planning D: Society and Space 28, 881-895 (2010).

253. Acedo, A., Painho, M. \& Casteleyn, S. Place and city: Operationalizing sense of place and social capital in the urban context. Transactions in GIS 21, 503-520 (2017).

254. Portela, M., Acedo, A. \& Granell-Canut, C. Looking for "in-between" Places. Media Theory 2, 108-133. ISSN: 2557-826X (2018).

255. Najafi, M. \& Shariff, M. The concept of place and sense of place in architectural studies. International Journal of Human and Social Sciences 6, 187-193 (2011).

256. Gotham, K. F. \& Brumley, K. Using space: Agency and identity in a publichousing development. City \& Community 1, 267-289 (2002).

257. Jorgensen, B. S. \& Stedman, R. C. Sense of place as an attitude: Lakeshore owners attitudes toward their properties. Journal of environmental psychology 21, 233-248 (2001).

258. Gaudiano, B. A. Cognitive-behavioral therapies: Achievements and challenges. Evidence-Based Mental Health 11, 5 (2008).

259. Andone, I. et al. How age and gender affect smartphone usage in Proceedings of the 2016 ACM International Joint Conference on Pervasive and Ubiquitous Computing: Adjunct (2016), 9-12.

260. Zeng, E. Y., Vilardaga, R., Heffner, J. L., Mull, K. E. \& Bricker, J. B. Predictors of utilization of a novel smoking cessation smartphone app. Telemedicine and e-Health 21, 998-1004 (2015).

261. Nathan, P. E. \& Gorman, J. M. A guide to treatments that work (Oxford University Press, 2015).

262. Barlow, D. H., Allen, L. B. \& Choate, M. L. Toward a unified treatment for emotional disorders. Behavior therapy 35, 205-230 (2004). 
263. Ellard, K. K., Fairholme, C. P., Boisseau, C. L., Farchione, T. J. \& Barlow, D. H. Unified protocol for the transdiagnostic treatment of emotional disorders: Protocol development and initial outcome data. Cognitive and Behavioral Practice 17, 88-101 (2010).

264. Turkington, D. et al. A training model for relatives and friends in cognitive behaviour therapy (CBT) informed care for psychosis. Cogent Psychology 5, 1-15 (2018).

265. Echeburúa, E., Gómez, M. \& Freixa, M. Cognitive-behavioural treatment of pathological gambling in individuals with chronic schizophrenia: A pilot study. Behaviour Research and Therapy 49, 808-814 (2011).

266. Seaborn, K. \& Fels, D. I. Gamification in theory and action: A survey. International Journal of human-computer studies 74, 14-31 (2015).

267. Nacke, L. E. \& Deterding, C. S. The maturing of gamification research. Computers in Human Behaviour, 450-454 (2017).

268. Bartle, R. Hearts, clubs, diamonds, spades: Players who suit MUDs. Journal of MUD research 1, 19 (1996).

269. Tondello, G. F., Wehbe, R. R., Orji, R., Ribeiro, G. \& Nacke, L. E. A Framework and Taxonomy of Videogame Playing Preferences in Proceedings of the Annual Symposium on Computer-Human Interaction in Play (2017), 329-340.

270. Ballatore, A. Prolegomena for an ontology of place in Advancing geographic information science (GSDI Association Press Needham, MA, 2016), 91103.

271. Huang, H., Gartner, G., Krisp, J. M., Raubal, M. \& Van de Weghe, N. Location based services: ongoing evolution and research agenda. Journal of Location Based Services 12, 63-93 (2018).

272. Marley, J. \& Farooq, S. Mobile telephone apps in mental health practice: uses, opportunities and challenges. BJPsych bulletin 39, 288-290 (2015).

273. Olff, M. Mobile mental health: A challenging research agenda. European Journal of Psychotraumatology 6, 27882 (2015). 
274. Sharma, H. N., Alharthi, S. A., Dolgov, I. \& Toups, Z. O. A framework supporting selecting space to make place in spatial mixed reality play in Proceedings of the Annual Symposium on Computer-Human Interaction in Play (2017), 83-100.

275. Miller, H. J. \& Tolle, K. Big data for healthy cities: Using location-aware technologies, open data and 3D urban models to design healthier built environments. Built Environment 42, 441-456 (2016).

276. Alegre, U., Augusto, J. C. \& Clark, T. Engineering context-aware systems and applications: A survey. Journal of Systems and Software 117, 55-83 (2016).

277. Hung, G. C.-L., Yang, P.-C., Wang, C.-Y. \& Chiang, J.-H. A SmartphoneBased Personalized Activity Recommender System for Patients with Depression in Proceedings of the 5th EAI International Conference on Wireless Mobile Communication and Healthcare (2015), 253-257.

278. Wahle, F., Kowatsch, T., Fleisch, E., Rufer, M. \& Weidt, S. Mobile sensing and support for people with depression: a pilot trial in the wild. JMIR mHealth and uHealth 4 (2016).

279. Simm, W., Ferrario, M. A., Gradinar, A. \& Whittle, J. Prototyping 'clasp': implications for designing digital technology for and with adults with autism in Proceedings of the 2014 conference on Designing interactive systems (2014), 345-354.

280. Mandryk, R. L. \& Birk, M. V. Toward game-based digital mental health interventions: player habits and preferences. Journal of Medical Internet Research 19 (2017).

281. Gordon, M. et al. Participatory design of ehealth solutions for women from vulnerable populations with perinatal depression. Journal of the American Medical Informatics Association 23, 105-109 (2015).

282. Acedo, A., Painho, M., Casteleyn, S. \& Roche, S. Place and City: Toward Urban Intelligence. ISPRS International Journal of Geo-Information 7, 22. ISSN: 2220-9964 (1st Jan. 2018).

283. Matthews, M., Abdullah, S., Gay, G. \& Choudhury, T. Tracking mental wellbeing: Balancing rich sensing and patient needs. Computer, 36-43 (2014). 
284. World Health Organization. Depression 2018. https://www . who. int/newsroom/fact-sheets/detail/depression (2018).

285. Association, A. P. et al. Diagnostic and statistical manual of mental disorders (DSM-5®) (American Psychiatric Pub, 2013).

286. Dimidjian, S., Martell, C. R., Addis, M. E., Herman-Dunn, R. \& Barlow, D. Behavioral activation for depression. Clinical handbook of psychological disorders: A step-by-step treatment manual 4, 328-364 (2008).

287. Spates, C. R., Pagoto, S. L. \& Kalata, A. A qualitative and quantitative review of behavioral activation treatment of major depressive disorder. The Behavior Analyst Today 7, 508 (2006).

288. Ekers, D. et al. Behavioural activation for depression; an update of metaanalysis of effectiveness and sub group analysis. PLOS ONE 9, e100100 (2014).

289. Depression, N. Management of depression in primary and secondary care. London: National Collaborating Centre for Mental Health and The Royal College of Psychiatrists' Research Unit ed. National Clinical Practice Guideline (2004).

290. Thomée, S. Mobile Phone Use and Mental Health. A Review of the Research That Takes a Psychological Perspective on Exposure. International journal of environmental research and public health 15, 2692 (2018).

291. I, M. et al. title. JMIR Preprints (2019).

292. Arean, P. A. et al. The use and effectiveness of mobile apps for depression: results from a fully remote clinical trial. Journal of medical Internet research 18 (2016).

293. Fitzpatrick, K. K., Darcy, A. \& Vierhile, M. Delivering cognitive behavior therapy to young adults with symptoms of depression and anxiety using a fully automated conversational agent (Woebot): a randomized controlled trial. JMIR Mental Health 4 (2017).

294. Hur, J.-W., Kim, B., Park, D. \& Choi, S.-W. A Scenario-Based Cognitive Behavioral Therapy Mobile App to Reduce Dysfunctional Beliefs in Individuals with Depression: A Randomized Controlled Trial. Telemedicine and e-Health (2018). 
295. Fleming, T. M. et al. Serious games for the treatment or prevention of depression: a systematic review. Revista de Psicopatología y Psicología Clínica 19, 227-242 (2014).

296. Stallard, P., Richardson, T., Velleman, S. \& Attwood, M. Computerized CBT (Think, Feel, Do) for depression and anxiety in children and adolescents: outcomes and feedback from a pilot randomized controlled trial. Behavioural and cognitive psychotherapy 39, 273-284 (2011).

297. Lucassen, M. F., Merry, S. N., Hatcher, S. \& Frampton, C. M. Rainbow SPARX: A novel approach to addressing depression in sexual minority youth. Cognitive and Behavioral Practice 22, 203-216 (2015).

298. Stasiak, K. et al. The journey towards new generation e-therapy for adolescents with depression. Neuropsychiatrie de l'Enfance et de l'Adolescence 5, S144 (2012).

299. Burns, J. M., Webb, M., Durkin, L. A. \& Hickie, I. B. Reach Out Central: a serious game designed to engage young men to improve mental health and wellbeing. Medical Journal of Australia 192, S27-S30 (2010).

300. Miralles, I. \& Granell, C. Considerations for Designing Context-Aware Mobile Apps for Mental Health Interventions. International Journal of Environmental Research and Public Health 16, 1197 (2019).

301. Rodríguez-Pupo, L., Casteleyn, S. \& Granell, C. On Metrics for LocationAware Games. ISPRS International Journal of Geo-Information 6, 299 (2017).

302. Schlieder, C., Kiefer, P. \& Matyas, S. Geogames: Designing location-based games from classic board games. IEEE Intelligent Systems 21, 40-46 (2006).

303. Schlatter, B. E. \& Hurd, A. R. Geocaching: 21st-century hide-and-seek. Journal of Physical Education, Recreation \& Dance 76, 28-32 (2005).

304. Southerton, C. Zombies, run!': Rethinking immersion in light of nontraditional gaming contexts. Transmedia: Storytelling and Beyond Digital Interfaces (2013).

305. Majorek, M. \& Du Vall, M. Ingress: an example of a new dimension in entertainment. Games and Culture 11, 667-689 (2016). 
306. Bartoschek, T., Schwering, A., Li, R., Münzer, S. \& Carlos, V. OriGami: a mobile geogame for spatial literacy in Geogames and Geoplay (Springer, 2018), 37-62.

307. Wars, T. Turf Wars 2019. https: //turfwarsapp. com/ (2019).

308. Ruiz-Ariza, A., Casuso, R. A., Suarez-Manzano, S. \& Martınez-López, E. J. Effect of augmented reality game Pokémon GO on cognitive performance and emotional intelligence in adolescent young. Computers \& Education 116, 49-63 (2018).

309. Merritt, T., Nielsen, C. L., Jakobsen, F. L. \& Grønbæk, J. E. Glowphones: designing for proxemics play with low-resolution displays in location-based games in Proceedings of the Annual Symposium on Computer-Human Interaction in Play (2017), 69-81.

310. Rosenqvist, R., Boldsen, J., Papachristos, E. \& Merritt, T. MeteorQuestBringing Families Together Through Proxemics Play In A Mobile Social Game in Proceedings of the 2018 Annual Symposium on Computer-Human Interaction in Play (2018), 439-450.

311. Bonsignore, E. et al. Alternate Reality Games: platforms for collaborative learning in Proceedings of the Tenth International Conference of the Learning Sciences (2012).

312. Spallazzo, D. \& Mariani, I. Location-Based Mobile Games (Springer, 2018).

313. Wahle, F., Kowatsch, T., Fleisch, E., Rufer, M. \& Weidt, S. Mobile sensing and support for people with depression: a pilot trial in the wild. JMIR mHealth and uHealth 4 (2016).

314. Hung, G. C.-L., Yang, P.-C., Wang, C.-Y. \& Chiang, J.-H. A SmartphoneBased Personalized Activity Recommender System for Patients with Depression in Proceedings of the 5th EAI International Conference on Wireless Mobile Communication and Healthcare (2015), 253-257.

315. Rising, L. \& Janoff, N. S. The Scrum software development process for small teams. IEEE software 17, 26-32 (2000).

316. LABPSITEC. Laboratorio de Psicología y Tecnología 2019. http://www . labpsitec.uji.es/esp/index.php (2019).

317. Google. Angular 2019. https://angular.io/ (2019). 
318. Labs, C. CoreUl 2019. https://coreui.io/ (2019).

319. Keßler, C. \& McKenzie, G. A geoprivacy manifesto. Transactions in GIS 22, 3-19 (2018).

320. Google. Google Play Location Services 2019. https://developer android. com/training/location (2019).

321. Gis, P. Spatial and Geographic Objects for PostgreSQL 2019. https : // postgis.net/ (2019).

322. Spanakis, E. G. et al. Technology-Based Innovations to Foster Personalized Healthy Lifestyles and Well-Being: A Targeted Review. Journal of Medical Internet Research 18, e128 (June 2016).

323. Bélanger, C. et al. Predictors of Dropout From Cognitive-Behavioral Group Treatment for Panic Disorder With Agoraphobia: An Exploratory Study. Behavior Modification 41, 113-140 (2017).

324. Hu, R. J. Diagnostic and Statistical Manual of Mental Disorders (DSM-IV). Encyclopedia of the Neurological Sciences, 4-8 (2003).

325. Barlow, D. H., Gorman, J. M., Shear, M. K. \& Woods, S. W. CognitiveBehavioral Therapy, Imipramine, or Their Combination for Panic Disorder: A Randomized Controlled Trial. JAMA 283, 2529-2536 (2000).

326. Craske, M. G., Brown, T. A. \& Barlow, D. H. Behavioral treatment of panic disorder: A two-year follow-up. Behavior Therapy 22, 289-304 (1991).

327. McHugh, R. K., Smits, J. A. \& Otto, M. W. Empirically supported treatments for panic disorder. Psychiatric Clinics of North America 32, 593-610 (2009).

328. McIntosh, A. et al. Clinical guidelines and evidence review for panic disorder and generalised anxiety disorder. Sheffield: University of Sheffield/London: National Collaborating Centre for Primary Care, 1-165 (2004).

329. Öst, L.-G., Thulin, U. \& Ramnerö, J. Cognitive behavior therapy vs exposure in vivo in the treatment of panic disorder with agrophobia. Behaviour Research and Therapy 42, 1105-1127 (2004).

330. Otto, M. W., Pollack, M. H. \& Maki, K. M. Empirically supported treatments for panic disorder: costs, benefits, and stepped care. Journal of Consulting and Clinical Psychology 68, 556-563 (2000). 
331. Chambless, D. L. \& Hollon, S. D. Defining Empirically Supported Therapies. Journal of Consulting and Clinical Psychology 66, 7-18 (1998).

332. Mitte, K. A meta-analysis of the efficacy of psycho- and pharmacotherapy in panic disorder with and without agoraphobia. Journal of Affective Disorders 88, 27-45 (2005).

333. Hofmann, S. G., Asnaani, A., Vonk, I. J. J., Sawyer, A. T. \& Fang, A. The Efficacy of Cognitive Behavioral Therapy: A Review of Meta-analyses. Cognitive Therapy and Research 36, 427-440 (2012).

334. Taylor, S. Understanding and Treating Panic Disorder: Cognitive-Behavioural Approaches (2000).

335. Olatunji, B. O., Deacon, B. J. \& Abramowitz, J. S. The Cruelest Cure? Ethical Issues in the Implementation of Exposure-Based Treatments. Cognitive and Behavioral Practice 16, 172-180 (2009).

336. Richard, D. C. \& Gloster, A. T. Exposure therapy has a public relations problem: A dearth of litigation amid a wealth of concern in (2007), 409-425.

337. Gloster, A. T. et al. Psychological Treatment for Panic Disorder with Agoraphobia: A Randomized Controlled Trial to Examine the Role of TherapistGuided Exposure in-situ in CBT. Journal of Consulting and Clinical Psychology 79, 406-420 (2011).

338. Powers, M. B., Smits, J. A. J. \& Telch, M. J. Disentangling the Effects of Safety-Behavior Utilization and Safety-Behavior Availability During ExposureBased Treatment: A Placebo-Controlled Trial. Journal of Consulting and Clinical Psychology 72, 448-454 (2004).

339. Mansell, W. \& McEvoy, P. M. A test of the core process account of psychopathology in a heterogenous clinical sample of anxiety and depression: A case of the blind men and the elephant? Journal of Anxiety Disorders 46, 4-10 (2017).

340. Barlow, D. et al. Unified Protocol for Transdiagnostic Treatment of Emotional Disorders: Therapist Guide (2010).

341. Dear, B. et al. Transdiagnostic versus disorder-specific and clinician-guided versus self-guided internet-delivered treatment for generalized anxiety disorder and comorbid disorders: A randomized controlled trial. Journal of Anxiety Disorders 36, 63-77 (2015). 
342. Dear, B. et al. Transdiagnostic versus disorder-specific and clinician-guided versus self-guided internet-delivered treatment for Social Anxiety Disorder and comorbid disorders: a randomized controlled trial. Journal of Anxiety Disorders 42, 30-44 (2016).

343. Farchione, T. J. et al. Unified protocol for transdiagnostic treatment of emotional disorders: a randomized controlled trial. Behavior Therapy 43, 666-678 (2012).

344. Fogliati, V. et al. Disorder-specific versus transdiagnostic and clinicianguided versus self-guided internet-delivered treatment for panic disorder and comorbid disorders: A randomized controlled trial. Journal of Anxiety Disorders 39, 88-102 (2016).

345. Norton, P. J. An Open Trial of a Transdiagnostic Cognitive-Behavioral Group Therapy for Anxiety Disorder. Behavior Therapy 39, 242-250 (2008).

346. Titov, N. et al. Disorder-specific versus transdiagnostic and clinician-guided versus self-guided treatment for major depressive disorder and comorbid anxiety disorders: A randomized controlled trial. Journal of Anxiety Disorders 35, 88-102 (2015).

347. Newby, J. M., McKinnon, A., Kuyken, W., Gilbody, S. \& Dalgleish, T. Systematic review and meta-analysis of transdiagnostic psychological treatments for anxiety and depressive disorders in adulthood. Clinical Psychology Review 40, 91-110 (2015).

348. Pearl, S. B. \& Norton, P. J. Transdiagnostic versus diagnosis specific cognitive behavioural therapies for anxiety: A meta-analysis. Journal of Anxiety Disorders 46, 11-24 (2017).

349. Reinholt, N. \& Krogh, J. Efficacy of Transdiagnostic Cognitive Behaviour Therapy for Anxiety Disorders: A Systematic Review and Meta-Analysis of Published Outcome Studies. Cognitive Behaviour Therapy 43, 171-184 (2014).

350. Vincelli, F. et al. Experiential cognitive therapy in the treatment of panic disorders with agoraphobia: a controlled study. Cyberpsychology, Behavior, and Social Networking 6, 321-328 (2003). 
351. Newby, J. M., Twomey, C., Li, S. S. Y. \& Andrews, G. Transdiagnostic computerised cognitive behavioural therapy for depression and anxiety: A systematic review and meta-analysis. Journal of Affective Disorders 199, 30-41 (2016).

352. Tulbure, B. T. A Web-Based Transdiagnostic Intervention for Affective and Mood Disorders: Randomized Controlled Trial. JMIR Mental Health 5 (2018).

353. González-Robles, A. et al. Eficacia de un protocolo de tratamiento transdiagnóstico online para los trastornos emocionales en el sistema público de salud mental. Congreso de la Eficacia a la efectividad: la psicología clínica al servicio de la ciudadanía (2017).

354. Santana, L. \& Fontenelle, L. F. A review of studies concerning treatment adherence of patients with anxiety disorders. Patient Preference and Adherence 5, 427-439 (2011).

355. Rush, B. \& Scott, R. Approved telehealth outcome indicator guidelines: quality, access, acceptability and cost. Calgary, AB, Canada: Calgary Health Telematics Unit, University of Calgary (2004).

356. Wallin, E. E. K., Mattsson, S. \& Olsson, E. M. G. The Preference for InternetBased Psychological Interventions by Individuals Without Past or Current Use of Mental Health Treatment Delivered Online: A Survey Study With Mixed-Methods Analysis. JMIR Mental Health 3 (2016).

357. Campos, D. et al. The acceptability of an Internet-based exposure treatment for flying phobia with and without therapist guidance: patients' expectations, satisfaction, treatment preferences, and usability. Neuropsychiatric Disease and Treatment 14, 879-892 (2018).

358. Greenberg, R. P., Constantino, M. J. \& Bruce, N. Are patient expectations still relevant for psychotherapy process and outcome. Clinical Psychology Review 26, 657-678 (2006).

359. Quero, S. et al. Acceptability of virtual reality interoceptive exposure for the treatment of panic disorder with agoraphobia. British Journal of Guidance \& Counselling 42, 123-137 (2014).

360. Martin, H. V., Botella, C., García-Palacios, A. \& Osma, J. Virtual Reality Exposure in the Treatment of Panic Disorder with Agoraphobia: A Case Study. Cognitive and Behavioral Practice 14, 58-69 (2007). 
361. Botella, C. et al. An Internet-based program for depression using activity and physiological sensors: efficacy, expectations, satisfaction, and ease of use. Neuropsychiatric Disease and Treatment 12, 393-406 (2016).

362. Anderson, P., Zimand, E., Schmertz, S. \& Ferrer, M. Usability and Utility of a Computerized Cognitive-Behavioral Self-Help Program for Public Speaking Anxiety. Cognitive and Behavioral Practice 14, 198-207 (2007).

363. Rizvi, S. L., Dimeff, L. A., Skutch, J. M., Carroll, D. A. \& Linehan, M. M. A Pilot Study of the DBT Coach: An Interactive Mobile Phone Application for Individuals with Borderline Personality Disorder and Substance Use Disorder. Behavior Therapy 42, 589-600 (2011).

364. Olbrich, H., Stengler, K. \& Olbrich, S. Smartphone based Geo-Feedback in obsessive compulsive disorder as facilitatory intervention: A case report. Journal of Obsessive-Compulsive and Related Disorders 8, 75-78 (2016).

365. Nardo, P. A. D., Moras, K., Barlow, D. H., Rapee, R. M. \& Brown, T. A. Reliability of DSM-III-R Anxiety Disorder Categories: Using the Anxiety Disorders Interview Schedule-Revised (ADIS-R). Archives of General Psychiatry 50, 251-256 (1993).

366. Marks, I. \& Mathews, A. Brief standard self-rating for phobic patients. Behaviour Research and Therapy 17, 263-267 (1979).

367. Borkovec, T. D. \& Nau, S. D. Credibility of analogue therapy rationales. Journal of Behavior Therapy and Experimental Psychiatry 3, 257-260 (1972).

368. Botella, C. et al. The acceptability of an Internet-based self-help treatment for fear of public speaking. British Journal of Guidance \& Counselling 37, 297-311 (2009).

369. Botella, C. et al. In Vivo versus Augmented Reality Exposure in the Treatment of Small Animal Phobia: A Randomized Controlled Trial. PLOS ONE 11 (2016).

370. Brooke, J. Sus: a 'quick and dirty' usability scale (2006).

371. Albert, W. \& Tullis, T. Measuring the user experience: collecting, analyzing, and presenting usability metrics (Newnes, 2013). 
372. Barlow, D. H. et al. Protocolo unificado para el tratamiento transdiagnóstico de los trastornos emocionales (Alianza Editorial, 2016).

373. Bangor, A., Kortum, P. T. \& Miller, J. T. An Empirical Evaluation of the System Usability Scale. International Journal of Human-computer Interaction 24, 574-594 (2008).

374. Mira, A. et al. Exploring the Relationship Between the Acceptability of an Internet-Based Intervention for Depression in Primary Care and Clinical Outcomes: Secondary Analysis of a Randomized Controlled Trial. Frontiers in Psychiatry 10 (2019).

375. Jokela, T., livari, N., Matero, J. \& Karukka, M. The Standard of UserCentered Design and the Standard Definition of Usability: Analyzing ISO 13407 against ISO 9241-11. AICPS (2003).

376. Erickson, D. H., Janeck, A. S. \& Tallman, K. A Cognitive-Behavioral Group for Patients With Various Anxiety Disorders. Psychiatric Services 58, 12051211 (2007).

377. White, K. S. et al. Attrition in a multicenter clinical trial for panic disorder. Journal of Nervous and Mental Disease 198, 665-671 (2010).

378. Diaz, L. et al. Enhancing stimulus control in the treatment of gambling disorder using location-based technologies in The 6th ESRII Conference. (2019).

379. Oromendia, P., Orrego, J., Bonillo, A. \& Molinuevo, B. Internet-based selfhelp treatment for panic disorder: a randomized controlled trial comparing mandatory versus optional complementary psychological support. Cognitive Behaviour Therapy 45, 270-286 (2016).

380. Dowling, N. A. et al. The prevalence of comorbid personality disorders in treatment-seeking problem gamblers: A systematic review and metaanalysis. Journal of Personality Disorders 29, 735-754 (2015).

381. Rash, C. J., Weinstock, J. \& Van Patten, R. A review of gambling disorder and substance use disorders. Substance abuse and rehabilitation 7, 3 (2016).

382. Rogier, G. \& Velotti, P. Narcissistic implications in gambling disorder: The mediating role of emotion dysregulation. Journal of gambling studies $\mathbf{3 4}$, 1241-1260 (2018). 
383. Bergamini, A. et al. At-risk gambling in patients with severe mental illness: Prevalence and associated features. Journal of behavioral addictions 7 , 348-354 (2018).

384. Desai, R. A. \& Potenza, M. N. A cross sectional study of problem and pathological gambling in patients with schizophrenia/schizoaffective disorder. The Journal of clinical psychiatry 70, 1250 (2009).

385. Hakansson, A., Karlsson, A. \& Widinghoff, C. Primary and secondary diagnoses of gambling disorder and psychiatric comorbidity in the Swedish health care system-a nationwide register study. Frontiers in psychiatry $\mathbf{9}$, 426 (2018).

386. Lorains, F. K., Cowlishaw, S. \& Thomas, S. A. Prevalence of comorbid disorders in problem and pathological gambling: Systematic review and meta-analysis of population surveys. Addiction 106, 490-498 (2011).

387. De Ordenación del Juego, D. G. Estudio sobre prevalencia, comportamiento y caracterısticas de los usuarios de juegos de azar en España 20152016.

388. Choi, S.-W. et al. Treatment modalities for patients with gambling disorder. Annals of general psychiatry 16, 23 (2017).

389. Cowlishaw, S. et al. Psychological therapies for pathological and problem gambling. Cochrane Database of Systematic Reviews (2012).

390. Menchon, J. M., Mestre-Bach, G., Steward, T., Fernández-Aranda, F. \& Jiménez-Murcia, S. An overview of gambling disorder: from treatment approaches to risk factors. F1000Research 7 (2018).

391. Goslar, M., Leibetseder, M., Muench, H. M., Hofmann, S. G. \& Laireiter, A.-R. Efficacy of face-to-face versus self-guided treatments for disordered gambling: A meta-analysis. Journal of behavioral addictions 6, 142-162 (2017).

392. Dowling, N., Jackson, A. C. \& Thomas, S. A. Behavioral interventions in the treatment of pathological gambling: A review of activity scheduling and desensitization. International journal of behavioral consultation and therapy 4, 172 (2008).

393. Riley, B. et al. Extinction of gambling cue-reactivity: A pilot study in a problem gambling treatment setting (2018). 
394. Tolchard, B. Cognitive-behavior therapy for problem gambling: A critique of current treatments and proposed new unified approach. Journal of Mental Health 26, 283-290 (2017).

395. Ginley, M. K., Rash, C. J. \& Petry, N. M. Psychological Interventions in Gambling Disorder in Gambling Disorder (Springer, 2019), 181-194.

396. Garcia-Palacios, A., de la Vega, N., Botella, C., Banos, R. \& Quero, S. Virtual reality in the treatment of pathological gambling in Cyberpsychology \& Behavior 9 (2006), 706-707.

397. Bouchard, S. et al. Using Virtual reality in the Treatment of gambling Disorder: The Development of a new Tool for cognitive Behavior Therapy. Frontiers in psychiatry 8, 27 (2017).

398. Bücker, L., Bierbrodt, J., Hand, I., Wittekind, C. \& Moritz, S. Effects of a depression-focused internet intervention in slot machine gamblers: $\mathrm{A}$ randomized controlled trial. PLOS ONE 13, e0198859 (2018).

399. Carlbring, P. \& Smit, F. Randomized trial of internet-delivered self-help with telephone support for pathological gamblers. Journal of consulting and clinical psychology 76, 1090 (2008).

400. Casey, L. M. et al. Internet-based delivery of cognitive behaviour therapy compared to monitoring, feedback and support for problem gambling: a randomised controlled trial. Journal of gambling studies 33, 993-1010 (2017).

401. Hodgins, D. C., Cunningham, J. A., Murray, R. \& Hagopian, S. Online selfdirected interventions for gambling disorder: Randomized controlled trial. Journal of gambling studies 35, 635-651 (2019).

402. Petry, N. M., Ginley, M. K. \& Rash, C. J. A systematic review of treatments for problem gambling. Psychology of Addictive Behaviors 31, 951 (2017).

403. Montañés, M. C. \& Llamas, J. “i Hagan juego, menores!”: frecuencia de juego en menores de edad y su relación con indicadores de adicción al juego. Revista española de drogodependencias, 34-47 (2017).

404. Iglesias, E. B. El juego patológico: prevalencia en España. Salud y drogas 4, 9-34 (2004). 
405. Gerstein, D. et al. Gambling impact and behavior study: Report to the national gambling impact study commission. Chicago: National Opinion Research Center (1999).

406. Marchica, L. \& Derevensky, J. L. Examining personalized feedback interventions for gambling disorders: A systematic review. Journal of behavioral addictions 5, 1-10 (2016).

407. Aragay, N. et al. Pathological gambling: Understanding relapses and dropouts. Comprehensive Psychiatry 57, 58-64 (2015).

408. Miralles, I., Granell, C. \& Huerta, J. Playability Index, Built Environment and Geo-Games Technology to Promoting Physical Activity in Urban Areas in Ubiquitous Computing and Ambient Intelligence (Springer, 2016), 437-444. 


\section{Appendix}

\section{A.1 Supplementary Material A: Keywords}

The list of keywords related to psychology were:

- mental (and also e-mental)

- psycholog* (i.e. psychology, psychological)

- psychiatric

- emotional

- health (and also e-health, u-health)

- treatment(s) (and also pretreatment, post-treatment)

- disorder(s)

- intervention(s)

- therapy(ies)

- distress,

- affection

- depressi* (i.e. depression, depressive)

- anxiety,

- ecological momentary intervention

The list of keywords related to computer science were: 
- cell (and cell-phone, cellphone)

- mobile

- smart (and smartphone)

- portable

- phone(s)

- device(s)

- $\operatorname{app}(\mathrm{s})$

- applicat* (i.e. application)

- mhealth

- uhealth

- ehealth

- emental

- android

- iphone

\section{A.2 Supplementary Material B: Search Queries}

Queries used boolean operators (AND, OR), proximity operators (NEAR/3, W/3) and wild characters supported by each citation database/information source. All searches were against title, keywords and abstract fields, and time frame was between 2013-2018.

\section{A.2.1 SCOPUS search}

We run one search against SCOPUS as below.

( TITLE-ABS-KEY ( ( ( cell OR mobile OR smartphone ${ }^{\star}$ OR smart OR portable ) W/3 ( phone* OR device* OR app OR apps OR applicat* ) ) OR mhealth OR uhealth OR ehealth OR emental OR android OR iphone OR mobile OR app OR 
apps) AND DOCTYPE ( ar OR cp ) AND PUBYEAR > 2012 ) AND ( TITLE-ABSKEY ( ( ( mental OR psycholog* OR psychiatric OR emotional) W/3 ( health OR treatment* OR disorder* OR intervention* OR therapy OR distress OR affection ) ) OR depressi* OR anxiety OR "Ecological Momentary Intervention" ) AND DOCTYPE ( ar OR cp ) AND PUBYEAR > 2012 AND LANGUAGE (english ))

\section{A.2.2 WoS searches}

We run a search with the computer science related keywords (WoS Search \#1) and a second one with the psychology related keywords (WoS Search \#2). The third search (WoS Search \#3) performed the intersection of the previous two. All searches used the following WoS collections /indices: CORE collection, Science Citation Index Expanded (SCI-EXPANDED) -1900-presente, Social Sciences Citation Index (SSCl) -1956-present, Arts and Humanities Citation Index (A\&HCl) -1975-present, Conference Proceedings Citation Index- Science (CPCI-S) -1990present, Conference Proceedings Citation Index- Social Science and Humanities (CPCl-SSH) -1990-present, Emerging Sources Citation Index (ESCI) -2015present.

- WoS Search \#1:

(((TS=)(( ( cell OR mobile OR smartphone* OR smart OR portable ) NEAR/3 (

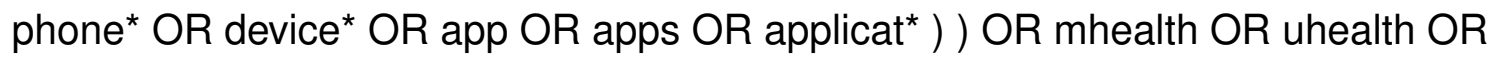
ehealth OR emental OR android OR iphone OR mobile OR app OR apps )))) AND Language: (English) AND Document Types: (Article OR Proceedings Paper)

- WoS Search \#2:

(((TS $=((($ mental OR psycholog* OR psychiatric OR emotional ) NEAR/3 ( health OR treatment ${ }^{\star}$ OR disorder* OR intervention* OR therapy OR distress OR affection ) ) OR depressi* OR anxiety OR "Ecological Momentary Intervention" )))) AND Language: (English) AND Document Types: (Article OR Proceedings Paper)

- WoS Search \#3:

(\#1 AND \#2) AND Language: (English) AND Document Type: (Article OR Proceedings Paper) 


\section{A.2.3 MEDLINE searches}

Like in WoS searchers, we conducted three separated queries to get the record form MEDLINE that satisfied both set of keywords. All searches used the MEDLINE collection/index.

- MEDLINE Search \#1:

(((TS=)( ( cell OR mobile OR smartphone* OR smart OR portable ) NEAR/3 ( phone* OR device* OR app OR apps OR applicat* ) ) OR mhealth OR uhealth OR ehealth OR emental OR android OR iphone OR mobile OR app OR apps ))))) AND Language:(English) AND Document Types: (Classical Article OR Congresses OR Journal Article)

- MEDLINE Search \#2:

( (((TS $=((($ mental OR psycholog* OR psychiatric OR emotional ) NEAR/3 ( health OR treatment* OR disorder* OR intervention* OR therapy OR distress OR affection ) ) OR depressi* OR anxiety OR "Ecological Momentary Intervention" ))))) AND Language: (English) AND Document Types: (Classical Article OR Congresses OR Journal Article)

- MEDLINE Search \#3:

(\#1 AND \#2) AND Language: (English) AND Document Types: (Classical Article OR Congresses OR Journal Article)

\section{A.2.4 APA PsycNET search}

As proximity operators are not supported, we combined multiple "AND" and "OR" operators to partially reflect proximity operators. Obviously, we cannot fully emulate the semantics of the proximity operators using only boolean operators. PsycNEt does not support the option (in the query search user interface) for searching "Conference papers", so we limited the search to "Journal Papers" and "Peer Reviewed Journals". NOTE: the above search does not work directly in the search text box. 
$(((($ title: (mhealth))) OR ((Keywords: (mhealth))) OR ((abstract: (mhealth))) OR ((title: (uhealth))) OR ((Keywords: (uhealth))) OR ((abstract: (uhealth))) OR ((title: (ehealth))) OR ((Keywords: (ehealth))) OR ((abstract: (ehealth))) OR ((title: (emental))) OR ((Keywords: (emental))) OR ((abstract: (emental))) OR ((title: (android))) OR ((Keywords: (android))) OR ((abstract: (android))) OR ((title: (iphone))) OR ((Keywords: (iphone))) OR ((abstract: (iphone)))OR ((title: (mobile))) OR ((Keywords: (mobile))) OR ((abstract: (mobile))) OR ((title: (app))) OR ((Keywords: (app))) OR ((abstract: (app))) OR ((title: (apps))) OR ((Keywords: (apps))) OR ((abstract: (apps)))) AND ((Year: [2013 TO 2018]) AND PublicationTypeFilt: ("Peer Reviewed Journal") AND ((DocumentType: ("Journal Article"))))) OR ((((((title: (cell)))) OR (((Keywords: (cell)))) OR (((abstract: (cell)))) OR (((title: (mobile)))) OR $((($ Keywords: (mobile $))))$ OR $((($ abstract: $($ mobile $))))$ OR $\left(\left(\left(\right.\right.\right.$ title: $\left(\right.$ smartphone $\left.\left.\left.\left.^{*}\right)\right)\right)\right)$ OR $\left(\left(\left(\right.\right.\right.$ Keywords: $\left(\right.$ smartphone $\left.\left.\left.\left.^{*}\right)\right)\right)\right)$ OR $\left(\left(\left(\right.\right.\right.$ abstract: $\left(\right.$ smartphone $\left.\left.\left.\left.^{*}\right)\right)\right)\right)$ OR $((($ title: (smart)))) OR (((Keywords: (smart)))) OR (((abstract: (smart)))) OR (((title: (portable $))))$ OR $((($ Keywords: (portable $))))$ OR $((($ abstract: (portable $)))))$ AND $(($ Year: [2013 TO 2018]) AND (PublicationTypeFilt: ("Peer Reviewed Journal")) AND (((DocumentType: ("Journal Article"))))) AND $\left(\left(\left(\left(\left(\right.\right.\right.\right.\right.$ title: $\left(\right.$ phone $\left.\left.\left.\left.{ }^{*}\right)\right)\right)\right)$ OR $((($ Keywords: $\left(\right.$ phone $\left.\left.\left.\left.^{*}\right)\right)\right)\right)$ OR $\left(\left(\left(\right.\right.\right.$ abstract: $\left(\right.$ phone $\left.\left.\left.\left.^{*}\right)\right)\right)\right)$ OR $\left(\left(\left(\right.\right.\right.$ title: $\left(\right.$ device $\left.\left.\left.\left.{ }^{*}\right)\right)\right)\right)$ OR $((($ Keywords: $\left(\right.$ device $\left.\left.\left.\left.{ }^{\star}\right)\right)\right)\right)$ OR $\left(\left(\left(\right.\right.\right.$ abstract: $\left(\right.$ device $\left.\left.\left.\left.^{*}\right)\right)\right)\right)$ OR $((($ title: $($ app $))))$ OR $((($ Keywords: (app)))) OR (((abstract: (app)))) OR (((title: (apps)))) OR (((Keywords: (apps)))) OR $((($ abstract: $($ apps $))))$ OR $\left(\left(\left(\right.\right.\right.$ title: $\left(\right.$ applicat $\left.\left.\left.\left.{ }^{\star}\right)\right)\right)\right)$ OR $\left(\left(\left(\right.\right.\right.$ Keywords: $\left(\right.$ applicat $\left.\left.\left.\left.^{\star}\right)\right)\right)\right)$ OR (((abstract: (applicat $\left.\left.\left.\left.{ }^{\star}\right)\right)\right)\right)$ AND ((Year: [2013 TO 2018]) AND(PublicationTypeFilt: ("Peer Reviewed Journal")) AND (((DocumentType: ("Journal Article"))))))))AND $(((((($ title: (mental) $))))$ OR $((($ Keywords: (mental) $)))$ OR $((($ abstract: (mental $))))$ OR $\left(\left(\left(\right.\right.\right.$ title: $\left(\right.$ psycholog $\left.\left.\left.\left.{ }^{*}\right)\right)\right)\right)$ OR $\left(\left(\left(\right.\right.\right.$ Keywords: $\left(\right.$ psycholog $\left.\left.\left.\left.{ }^{*}\right)\right)\right)\right)$ OR $((($ abstract: (psycho$\left.\left.\left.\left.\log ^{*}\right)\right)\right)\right)$ OR $((($ title: (psychiatric $\left.)))\right)$ OR $((($ Keywords: (psychiatric) $)))$ OR $((($ abstract: (psychiatric) $)))$ OR $((($ title: (emotional) $)))$ OR $((($ Keywords: (emotional) $)))$ OR $((($ abstract: (emotional))))) AND ((Year: [2013 TO 2018]) AND (PublicationTypeFilt: ("Peer Reviewed Journal")) AND (((DocumentType: ("Journal Article")))))) AND $(((($ title: (health) $)))$ OR $((($ Keywords: (health))) ) OR $((($ abstract: (health) $)))$ OR $\left(\left(\left(\right.\right.\right.$ title: $\left(\right.$ treatment $\left.\left.\left.\left.^{\star}\right)\right)\right)\right)$ OR $\left(\left(\left(\right.\right.\right.$ Keywords: $\left(\right.$ treatment $\left.\left.\left.\left.^{\star}\right)\right)\right)\right)$ OR $((($ abstract: (treatment $\left.\left.\left.\left.^{\star}\right)\right)\right)\right)$ OR $\left(\left(\left(\right.\right.\right.$ title: $\left(\right.$ disorder $\left.\left.\left.\left.^{\star}\right)\right)\right)\right)$ OR $\left(\left(\left(\right.\right.\right.$ Keywords: (disorder $\left.\left.\left.\left.{ }^{\star}\right)\right)\right)\right)$ OR $((($ abstract: $\left(\right.$ disorder $\left.\left.\left.\left.^{*}\right)\right)\right)\right)$ OR $\left(\left(\left(\right.\right.\right.$ title: (intervention $\left.\left.\left.\left.{ }^{*}\right)\right)\right)\right)$ OR $((($ Keywords: (intervention*) $)))$ OR $((($ abstract: (intervention* $))))$ OR $((($ title: (therapy) $)))$ OR $((($ Keywords: (therapy $))))$ OR (((abstract: (therapy)))) OR (((title: (distress)))) OR (((Keywords: (distress)))) 
OR (((abstract: (distress)))) OR (((title: (affection)))) OR (((Keywords: (affection)))) OR (((abstract: (affection))))) AND ((Year: [2013 TO 2018]) AND (PublicationTypeFilt: ("Peer Reviewed Journal")) AND (((DocumentType: ("Journal Article")))))) OR ((((title: (depressi* $\left.\left.\left.{ }^{*}\right)\right)\right)$ OR $\left(\left(\right.\right.$ Keywords: $\left(\right.$ depressi $\left.\left.\left.{ }^{\star}\right)\right)\right)$ OR ((abstract: (depressi $\left.\left.\left.{ }^{\star}\right)\right)\right)$ OR ((title: (anxiety))) OR ((Keywords: (anxiety))) OR ((abstract: (anxiety))) OR ((title: ("Ecological Momentary Intervention"))) OR ((Keywords: ("Ecological Momentary Intervention"))) OR ((abstract: ("Ecological Momentary Intervention")))) AND ((Year: [2013 TO 2018]) AND PublicationTypeFilt: ("Peer Reviewed Journal") AND ((DocumentType: ("Journal Article"))))))AND Language: english

Summary of records initially identified through the above searches (March 9th, 2018), per citation database and year:

\begin{tabular}{|c|r|r|r|r|r|}
\hline Year & SCOPUS & WoS & MEDLINE & PsychNET & TOTAL \\
\hline 2018 & 96 & 79 & 135 & 28 & 338 \\
\hline 2017 & 607 & 573 & 537 & 324 & 2041 \\
\hline 2016 & 570 & 627 & 429 & 340 & 1966 \\
\hline 2015 & 481 & 446 & 285 & 268 & 1480 \\
\hline 2014 & 445 & 317 & 276 & 216 & 1254 \\
\hline 2013 & 306 & 228 & 162 & 158 & 854 \\
\hline $\mathbf{2 0 1 3 - 2 0 1 8}$ & 2505 & 2270 & 1824 & 1334 & 7933 \\
\hline
\end{tabular}

